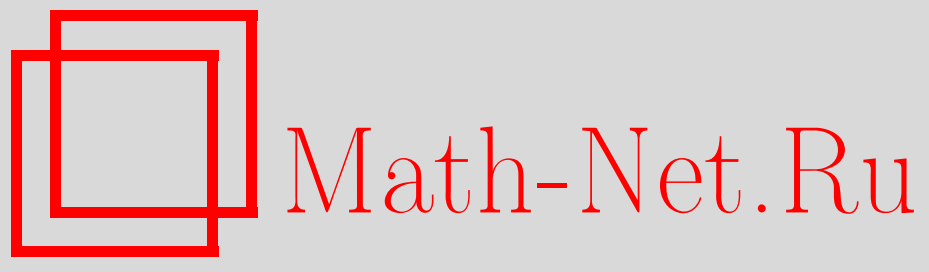

С. А. Мелихов, Стинродовские гомотопии, УМН, 2009, том 64, выпуск 3, 73-166

DOI: https://doi.org/10.4213/rm9284

Использование Общероссийского математического портала Math-Net.Ru подразумевает, что вы прочитали и согласны с пользовательским соглашением http://www . mathnet.ru/rus/agreement

Параметры загрузки:

IP : 52.23 .180 .231

26 апреля 2023 г., 15:01:50

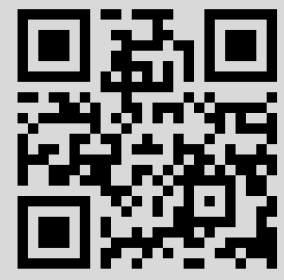




\section{Стинродовские гомотопии}

\section{С. А. Мелихов}

Теория стинродовских гомотопий занимается построением алгебраической топологии общих пространств в терминах алгебраической топологии полиэдров; а с другой точки зрения - изучением топологии функтора $\lim ^{1}$ (для обратных последовательностей групп). В настоящей статье наибольшее внимание уделено случаю компактов, в котором стинродовские гомотопии совпадают с сильным шейпом. Предпринята попытка упростить основания теории и прояснить и усилить некоторые из ее главных результатов.

Используя геометрические методы, такие как компактифицированный телескоп Милнора, комногообразия (mock bundles) и конструкцию Понтрягина-Тома, мы получаем новые простые доказательства теорем Баррата-Милнора, Гэгана-Красинкевича, Дыдака, Дыдака-Сигала, Красинкевича-Минца, Кэйти, Мардешича, Миттаг-Леффлера-Бурбаки, Фокса, Эды-Кавамуры, Эдвардса-Гэгана, Юссилы и трех неопубликованных теорем Щепина. Исправлена ошибка в доказательстве Лисицы “теоремы Гуревича для стинродовских гомотопий". Показано, что над компактами наложения (overlays) в смысле Фокса эквивалентны равномерным накрытиям в смысле Джеймса. В числе других результатов выделим следующие.

- Морфизм между обратными последовательностями счетных (быть может, неабелевых) групп, индуцирующий изоморфизмы на $\lim$ и $\lim ^{1}$, обратим в про-категории. Это влечет “теорему Уайтхеда для стинродовских гомотопий", тем самым доставляя ответ на два вопроса А. Коямы.

- Если $X$ - локально $(n-1)$-связный компакт, $n \geqslant 1$, то его $n$-мерные стинродовские гомотопические классы представимы отображениями $S^{n} \rightarrow X$ при условии, что $X$ односвязен. Предположение односвязности нельзя опустить в силу известного примера Дыдака и Здравковской.

- Связный компакт связен по Стинроду (pointed 1-movable), если и только если всякое его равномерное накрывающее пространство имеет счетное количество компонент равномерной связности.

Библиография: 117 названий.

Ключевые слова: компактифицированный телескоп, комногообразия, конструкция Понтрягина-Тома, наложения, равномерные накрытия, шейповая 1-подвижность, функтор производного предела, стинродовские гомотопии, стинродовские гомологии, сильный шейп.

Работа выполнена при поддержке РФФИ (грант 08-01-00663-а), гранта Президента РФ MK-5411.2007.1 и программы ОМ РАН “Современные проблемы теоретической математики".

(C) С. А. МЕлихов, 2009 


\section{СОДЕРЖАНИЕ}

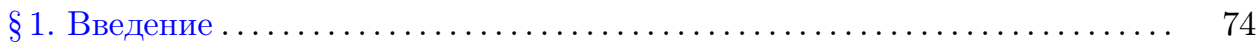

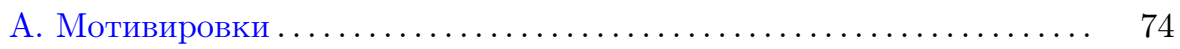

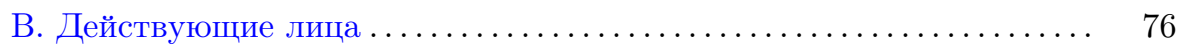

C. Стинродовские теории против сингулярных ............... 79

D. Сравнение с литературой ........................ 81

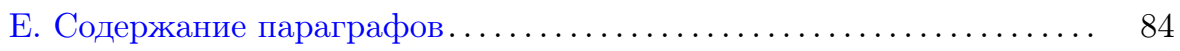

$\S 2$. Стинродовская гомотопическая категория.................. 87

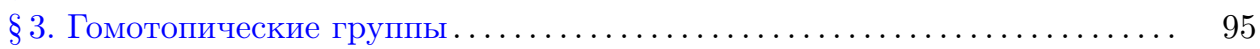

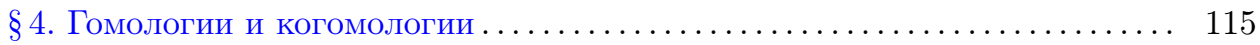

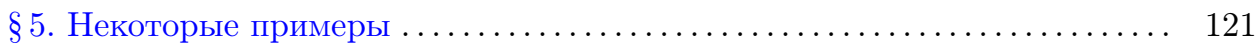

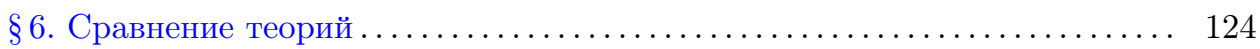

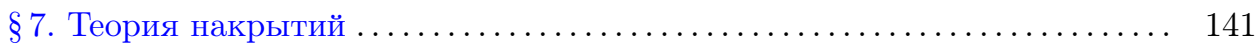

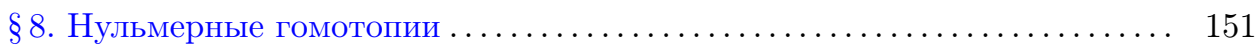

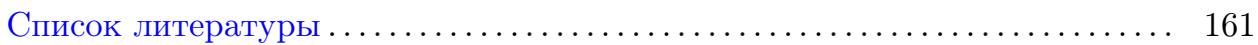

\section{§ 1. Введение}

Перефразируя одно известное высказывание А. Н. Уайтхеда, можно заявить, что конечная цель теории стинродовских гомотопий состоит в том, чтобы устранить всякую необходимость в общей топологии - сведя ее к комбинаторике. Если выражаться менее образно, теория стинродовских гомотопий - естественная общая схема построения алгебраической топологии весьма общих пространств в терминах алгебраической топологии полиэдров. Основная задача настоящих заметок - предоставить прозрачное построение теории стинродовских гомотопий компактов, т. е. компактных метризуемых пространств. Набросок распространения на конечномерно-аппроксимируемые (finitistic) метризуемые полные равномерные пространства приведен в конце $§ 7$. Изложение по существу замкнуто.

А. Мотивировки. Интерес автора к стинродовским гомотопиям был мотивирован вопросами, возникающими из других наук.

1) Две проблемы Д. Ролфсена (1972 г.) о топологической изотопии (т. е. гомотопии в классе вложений) в $\mathbb{R}^{3}$ : существует ли узел, не изотопный тривиальному? (Такой узел имеет шансы найтись лишь среди диких, поскольку всякий кусочно линейный узел изотопен тривиальному кусочно линейной изотопией, не являющейся локально-плоской.) Будут ли изотопные кусочно линейные зацепления кусочно линейно изотопными? Положительный ответ на последний вопрос вытекал бы из справедливости аналога гипотезы Васильева (о полноте инвариантов конечного порядка для узлов) для “зацеплений по модулю узлов" (т.е. кусочно линейных зацеплений, рассматриваемых с точностью до кусочно линейной изотопии) [1].

В целом, проблемы Ролфсена естественно рассматривать как более наглядные аналоги гипотезы Васильева, проясняющие геометрический смысл (см. [1]) препятствия типа $\lim ^{1}$ к справедливости последней. Такое препятствие $\theta(f, g)$, 
определенное для всякой пары узлов $f, g$, неразличимых инвариантами конечного порядка, может быть получено из переформулировки инвариантов конечного порядка в терминах изовариантной теории гомотопий подходящим образом компактифицированных конфигурационных пространств ${ }^{1}$; кроме того, как автор узнал из разных источников, некое подобное препятствие, по всей видимости, уже рассматривалось Р. Милгремом в 90-е годы (неопубликовано). Между прочим, надо думать, что классик теории узлов Ралф Фокс имел в виду очень близкие соображения, когда в своих последних двух статьях переключился на стинродовские гомотопии (см. §7).

2) Проблема Борсука о вложимости $n$-мерных абсолютных ретрактов в $\mathbb{R}^{2 n}$. Согласно классическому результату Щепина-Штанько, вложимость заданного $n$-мерного компакта $X$ в $\mathbb{R}^{m}$ в коразмерности $m-n \geqslant 3$ эквивалентна собственной вложимости подходящего бесконечного телескопа $P_{[0, \infty)}($ см. $\S 2)$ в $\mathbb{R}^{m} \times[0, \infty)$; см. точную формулировку в [10]. В метастабильном ранге $m>3(n+1) / 2$ к такой задаче вложимости имеется полное препятствие в эквивариантных стабильных когомотопиях, которое при $m=2 n$ сводится к препятствию в ординарных когомологиях [10]. В частности, оказывается, что влияние условия $\mathrm{LC}_{\infty}$ для $X$ на вложимость $X$ в $\mathbb{R}^{2 n}$ тесно связано с некоторой подгруппой в чеховских $2 n$-мерных когомологиях $X \times X \backslash \Delta$, отвечающей за неперестановочность $\lim$ (возникающего по причине некомпактности $X \times X \backslash \Delta$ ) и $\lim$ (возникающего потому, что $X$ не является полиэдром). Этой алгебры достаточно, чтобы построить контрпример к параметрической версии проблемы Борсука, т. е. $n$-мерный абсолютный ретракт, $n \geqslant 2$, допускающий неизотопные вложения в $\mathbb{R}^{2 n+1}$. Сама проблема Борсука, однако, оказывается намного более сложной.

3) Подход Р. Д. Эдвардса к проблеме Гильберта-Смита. Существует ли свободное действие группы $\mathbb{Z}_{p}$ целых $p$-адических чисел на $\mathrm{LC}_{\infty}$-компакте с конечномерным пространством орбит? Несложно переформулировать свойство $X$

\footnotetext{
${ }^{1}$ Приведем набросок построения. Если $M$ - гладкое многообразие, то конфигурационное пространство $M^{(r)}:=M^{r} \backslash$ (все диагонали) имеет “линейную" компактификацию $M^{[r]}$ Фалтона-Макфирсона (см. [2]), а также "полиномиальную" компактификацию $M^{\{r\}}$, получающуюся из $M^{[r]}$ раздутием подмногообразий все более вырожденных (в смысле корангов представляющих наборов векторов) предельных конфигураций [3]. Всякий кусочно линейный или кусочно гладкий узел $f: S^{1} \hookrightarrow S^{3}$ индуцирует $S_{r}$-эквивариантное сохраняющее страты отображение $f^{[r]}:\left(S^{1}\right)^{[r]} \rightarrow\left(S^{3}\right)^{[r]}$, и если $f$ гладкий, то $f^{[r]}$ "выпрямлено" ("aligned") в смысле Синхи (см. [4]), или, что эквивалентно, поднимается в $S_{r}$-эквивариантное сохраняющее страты отображение $f^{\{r\}}:\left(S^{1}\right)^{\{r\}} \rightarrow\left(S^{3}\right)^{\{r\}}$, где $\left(S^{1}\right)^{\{r\}}=\left(S^{1}\right)^{[r]}$, поскольку $S^{1}$ одномерна. Из результатов Синхи [2] и Волича [5], [6] теперь вытекает, что гладкие узлы $f$ и $g$ неразличимы рациональными инвариантами Васильева, если $f^{\{r\}}$ и $g^{\{r\}}$ гомотопны в пространстве $V_{r}$ всех $S_{r}$-эквивариантных сохраняющих страты отображений $\left(S^{1}\right)^{\{r\}} \rightarrow\left(S^{3}\right)^{\{r\}}$ для каждого $r$. (Весьма вероятно, что доказательство допускает уточнение, работающее над целыми числами, ср. [7]; к этому следует добавить, что до сих пор неизвестно, сводятся ли целочисленные инварианты Васильева к рациональным.) Обратно, если $f$ и $g$ неразличимы целочисленными инвариантами Васильева порядков $<r$, то в силу теоремы Гусарова [8] и Хабиро [9] они связаны посредством гладкой изотопии и $C_{r}$-движений. Легко видеть, что каждое $C_{r}$-движение неотличимо от изотопии с точки зрения любого набора $r$ точек на узле; это означает, что $f^{\{r\}}$ и $g^{\{r\}}$ связаны гомотопией $h_{r}: I \rightarrow V_{r}$. Наконец, если $f$ и $g$ неразличимы всеми целочисленными инвариантами Васильева, то каждая $h_{r}$ дополняется посредством $p_{r+1} h_{r+1}$, где $p_{r}: V_{r+1} \rightarrow V_{r}$ - забывающее отображение, до петли $\ell_{r}:\left(S^{1}, \mathrm{pt}\right) \rightarrow\left(V_{r}, f^{\{r\}}\right)$. Пусть $\theta(f, g)$ - класс $\left(\left[\ell_{1}\right],\left[\ell_{2}\right], \ldots\right)$ в $\lim ^{1} \pi_{1}\left(V_{r}, f^{\{r\}}\right)$. Если $f$ и $g$ связаны гладкой изотопией $\varphi_{t}$, то $p_{r+1} \varphi_{t}^{\{r+1\}}=\varphi_{t}^{\{r\}}$ для каждого $r$, поэтому $\theta(f, g)$ тривиально.
} 
быть пространством орбит свободного действия $\mathbb{Z}_{p}$ на некотором $\mathrm{LC}_{\infty}$-компакте в терминах локальных стинродовских гомотопий $X$. Это доставляет чисто топологическую (без упоминания групповых действий), причем даже комбинаторно-топологическую (ввиду природы стинродовских гомотопий) формулировку вышеприведенного вопроса.

В. Действующие лица. Пусть $X \subset \mathbb{R}^{m}$ - компакт. Стинродовское гомотопическое множество $\pi_{n}(X, x)$ - это множество классов эквивалентности сохраняющих уровни отображений

$$
F:\left(S^{n} \times[0, \infty), b \times[0, \infty)\right) \rightarrow\left(\mathbb{R}^{m} \times[0, \infty), x \times[0, \infty)\right)
$$

таких, что замыкание $F\left(S^{n} \times[0, \infty)\right)$ в $\mathbb{R}^{m} \times[0, \infty]$ пересекает $\mathbb{R}^{m} \times\{\infty\}$ по некоторому подмножеству компакта $X \times\{\infty\}$, относительно гомотопии в классе отображений того же вида. Как можно было ожидать, $\pi_{0}(X, x)$ - пунктированное множество, $\pi_{1}$ - группа (если принять во внимание базисный луч $b \times[0, \infty)$ ), а $\pi_{2}, \pi_{3}, \ldots$ - абелевы группы.

Пусть $\stackrel{\Delta}{\pi}_{n}(X, x)$ обозначает сингулярное гомотопическое множество, т. е. множество гомотопических классов отображений $\left(S^{n}, b\right) \rightarrow(X, x)$. Имеется гомоморфизм (пунктированное отображение при $n=0$ )

$$
\stackrel{\Delta}{\pi}_{n}(X, x) \stackrel{\stackrel{\Delta}{\tau}}{\rightarrow} \pi_{n}(X, x)
$$

сопоставляющий всякому сфероиду $f:\left(S^{n}, b\right) \rightarrow(X, x)$ стинродовский сфероид $f \times \operatorname{id}_{[0, \infty)}: S^{n} \times[0, \infty) \rightarrow X \times[0, \infty) \subset \mathbb{R}^{m} \times[0, \infty)$. Несложно видеть, что когда $X$ - полиэдр, $\stackrel{\Delta}{\tau}$ - изоморфизм.

Определение $\pi_{n}(X)$ можно сформулировать чисто комбинаторным образом, не упоминая замыкание. Возьмем последовательность $\cdots \subset P_{1} \subset P_{0}$ замкнутых полиэдральных окрестностей $X$ в $\mathbb{R}^{m}$ такую, что $P_{0}=\mathbb{R}^{m}$ и $\bigcap P_{i}=X$, и рассмотрим их телескоп

$$
P_{[0, \infty)}=\bigcup P_{i} \times[i, i+1],
$$

лежащий в $\mathbb{R}^{m} \times[0, \infty)$. Тогда $\pi_{n}(X, x)$ есть просто множество классов сохраняющей уровни гомотопии сохраняющих уровни отображений

$$
G:\left(S^{n} \times[0, \infty), b \times[0, \infty)\right) \rightarrow\left(P_{[0, \infty)}, x \times\{0, \infty\}\right) .
$$

(Действительно, всякое $G$ имеет вид $F$, а всякое $F$ можно привести к виду $G$ подходящей перепараметризацией $[0, \infty)$, ср. доказательство леммы 2.5.) Это частный случай определения из $\S 3$, где уже не потребуется вложения в $\mathbb{R}^{m}$, равно как и конечномерности $X$.

Сужая некоторое $G$ как выше на целочисленные уровни $S^{n} \times[0, \infty)$, мы получим набор отображений $g_{i}:\left(S^{n}, b\right) \rightarrow\left(P_{i}, x\right)$ таких, что каждое $g_{i+1}$ гомотопно $g_{i}$ со значениями в $P_{i}$. Этим описан эпиморфизм (пунктированная сюръекция при $n=0$ )

$$
\pi_{n}(X, x) \stackrel{\check{\tau}}{\rightarrow} \lim _{\longleftarrow} \pi_{n}\left(P_{i}, x\right) .
$$


Обратный предел ${ }^{2}$ в правой части известен также как "чеховская гомотопическая группа" $\check{\pi}_{n}(X, x)$. Хотя она является инвариантом $(X, x)$, как таковой она представляет ограниченный интерес, главным образом потому, что не удовлетворяет точной гомотопической последовательности пары - которой стинродовские гомотопические группы удовлетворяют. (Относительные стинродовские и чеховские гомотопические группы определяются без затруднений.)

Что представляет собой ядро $\check{\tau}$ ? Элемент этого ядра представи́м таким $G$, ограничение которого на каждый целочисленный уровень - постоянное отображение. Каждый $[i, i+1]$-слой $G$ задает тогда отображение $G_{i}:\left(S^{n+1}, b\right) \rightarrow$ $\left(P_{i}, x\right)$. Гомотопические классы этих сфероидов $G_{1}, G_{2}, \ldots$ определены, однако, не вполне корректно: мы можем отнять кусок (т. е. сфероид) от какогонибудь $G_{i}$ и прибавить его к $G_{i+1}$, не изменяя при этом класса $G$ в $\pi_{n}(X)$. Довольствуясь для простоты обозначений абелевым случаем $(n>0)$, мы заключаем, что ker $\check{\tau}-$ это в точности коядро гомоморфизма

$$
\varphi: \prod \pi_{n+1}\left(P_{i}\right) \rightarrow \prod \pi_{n+1}\left(P_{i}\right),
$$

определенного как $\left(\ldots, g_{1}, g_{0}\right) \mapsto\left(\ldots, g_{1}-f_{1}\left(g_{2}\right), g_{0}-f_{0}\left(g_{1}\right)\right)$, где гомоморфизмы $f_{i}: \pi_{n+1}\left(P_{i+1}\right) \rightarrow \pi_{n}\left(P_{i}\right)$ индуцированы включениями. Это коядро известно как производный предел $\lim ^{1} \pi_{n+1}\left(P_{i}\right)$ обратной последовательности наших абелевых групп. (Между прочим, заметим, что ядро $\varphi-$ не что иное, как $\lim _{n+1}\left(P_{i}\right)$.)

Итак, результаты наших наблюдений описываются (при $n>0$ ) короткой точной последовательностью

$$
0 \rightarrow \lim ^{1} \pi_{n+1}\left(P_{i}\right) \rightarrow \pi_{n}(X) \rightarrow \underset{\lim }{\longleftarrow} \pi_{n}\left(P_{i}\right) \rightarrow 0
$$

Ввиду незамысловатого характера обратного предела справа производный предел слева является, без сомнения, важнейшим алгебраическим объектом теории стинродовских гомотопий.

Рассмотрим теперь замкнутое (не обязательно ограниченное) подмножество $X$ в $\mathbb{R}^{m}$. Пусть $\cdots \subset P_{1} \subset P_{0}$ - замкнутые полиэдральные окрестности $X$ в $\mathbb{R}^{m}$ такие, что $P_{0}=\mathbb{R}^{m}$ и $\bigcap P_{i}=X$, причем при $i>0$ каждый $P_{i}$ содержится в $2^{-i}$-окрестности $X$ и содержит $2^{-(i+1)}$-окрестность $X$ относительно обычной евклидовой метрики на $\mathbb{R}^{m}$. Стинродовское гомотопическое множество $\pi_{n}(X, x)$ - это снова множество классов сохраняющей уровни гомотопии сохраняющих уровни отображений

$$
G:\left(S^{n} \times[0, \infty), b \times[0, \infty)\right) \rightarrow\left(P_{[0, \infty)}, x \times\{0, \infty\}\right)
$$

\footnotetext{
2 Обратный предел $\lim _{\longleftarrow} S_{i}$ последовательности $\cdots \stackrel{f_{1}}{\longrightarrow} S_{1} \stackrel{f_{0}}{\longrightarrow} S_{0}$ множеств и отображений это подмножество произведения $\prod S_{i}$, состоящее из всех последовательностей $\left(\ldots, s_{1}, s_{0}\right)$ таких, что $s_{i}=f_{i}\left(s_{i+1}\right)$ для всех $i$. Такие последовательности точек называются нитями, а последовательность множеств и отображений как выше - обратной последователъностъю; ее отображения называют связующими. Обратный предел групп (и гомоморфизмов) естественным образом оказывается группой, а обратный предел топологических пространств (и непрерывных отображений) - топологическим пространством. Например, $X$ гомеоморфен $\lim _{\longleftarrow}\left(\cdots \subset P_{1} \subset P_{0}\right)$ в описанной выше ситуации.
} 
в телескоп $P_{[0, \infty)}=\bigcup P_{i} \times[i, i+1]$. Если $X-$ равномерный полиэдр (см. [11]), например объединение кубов вида $\left[i_{1}, j_{1}\right] \times \cdots \times\left[i_{m}, j_{m}\right]$, где $i_{k}, j_{k} \in \mathbb{Z}$ (быть может, $\left.i_{k}=j_{k}\right)$, то естественный гомоморфизм $\stackrel{\Delta}{\pi}_{n}(X) \rightarrow \pi_{n}(X)-$ снова изоморфизм. Значит, в этом случае $\pi_{n}(X)$ - топологический инвариант $X$. В общем случае это не так: если $X$ - пара касающихся на бесконечности гипербол $\{(x, y) \mid y= \pm 1 / x\}$ в плоскости, то $\pi_{0}(X)=0$. Однако группа $\pi_{n}(X)-$ всегда равномерный инвариант $X$, т. е. инвариантна при гомеоморфизме, равномерно непрерывном в обоих направлениях (см. $§ 7$, где дано более общее определение $\pi_{n}(X)$, в котором уже не потребуется вложения в $\left.\mathbb{R}^{m}\right)$. Взамен наша $\pi_{n}(X)$ остается практически вычислимой, поскольку она по-прежнему включается в короткую точную последовательность $(*)$.

Сказанное применимо и к гомологиям, а также к когомологиям. Напомним, что если $K$ - локально-конечный симплициальный комплекс, то в дополнение к обычным симплициальным цепям $C_{*}(K)$ и коцепям $C^{*}(K)=\operatorname{Hom}\left(C_{*}(K), \mathbb{Z}\right)$ определены также кощепи с компактным носителем $C_{\mathrm{c}}^{*}(K)=\underline{\lim } C^{*}\left(K, K_{i}\right)$, где подкомплексы $\cdots \subset K_{1} \subset K_{0}$ имеют дополнения, состоящие из конечного числа симплексов, причем $\bigcap K_{i}=\varnothing,{ }^{3}$ а также локально-конечные ие$n и C_{*}^{\mathrm{lf}}(K)=\lim C_{*}\left(K, K_{i}\right) \simeq \operatorname{Hom}\left(C_{\mathrm{c}}^{*}(K), \mathbb{Z}\right)$. Локально-конечные гомологии $H_{n}^{\mathrm{lf}}(P)=H_{n}\left(\overleftarrow{C_{*}^{\mathrm{lf}}}(K)\right)$ локально-компактного полиэдра $P$, триангулированного комплексом $K$, и когомологии с компактным носителем $H_{\mathrm{lf}}^{n}(P)=H_{-n}\left(C_{\mathrm{c}}^{*}(K)\right)$ являются инвариантами собственной гомотопической эквивалентности. Двойственность Пуанкаре для ориентируемых $m$-многообразий, не предполагающихся компактными, имеет вид $H^{n}(M) \simeq H_{m-n}^{\text {lf }}(M), H_{n}(M) \simeq H_{\mathrm{c}}^{m-n}(M)$ (для многообразий с краем будет уже 4 разных изоморфизма).

Группы $H_{\mathrm{c}}^{n}$ и $H_{n}^{\mathrm{lf}}$ очень просто обобщаются на замкнутые подмножества евклидова пространства, если использовать прежние обозначения. Локально-конечные стинродовские гомологии $H_{n}^{\mathrm{lf}}(X)$ определяются как $H_{n+1}^{\mathrm{lf}}\left(P_{[0, \infty)}\right)$, а когомологии Понтрягина с компактным носителем $H_{\mathrm{c}}^{n}(X)$ - это группа $H_{\mathrm{c}}^{n+1}\left(P_{[0, \infty)}\right)$. Несложно показать, что, несмотря на то, что в их определении участвуют равномерные окрестности $P_{i}$ множества $X$, эти группы оказываются топологическими (а не только равномерными) инвариантами $X$. Однако, поскольку они не являются гомотопическими (а являются лишь собственными гомотопическими) инвариантами $X$, область их применения ограничена.

Обычные стинродовские гомологии $H_{n}(X)$ определяются как $(n+1)$-я группа гомологий комплекса "[0, [) -локально-конечных" цепей $C_{*}^{[0, \infty)}\left(P_{[0, \infty)}\right)=$ $\underset{\lim }{\longleftarrow} C_{*}\left(P_{[0, \infty)}, P_{[i, \infty)}\right)$, где $P_{[0, \infty)}$ триангулирован так, что каждый $P_{i}$ триангулируется подкомплексом, а через $P_{J}$ обозначен прообраз подмножества $J \subset[0, \infty)$ при проекции $P_{[0, \infty)} \rightarrow[0, \infty)$. Аналогично вышесказанному, имеет место естественная короткая точная последовательность

$$
0 \rightarrow \lim ^{1} H_{n+1}\left(P_{i}\right) \rightarrow H_{n}(X) \rightarrow \underset{\lim }{\longleftarrow} H_{n}\left(P_{i}\right) \rightarrow 0
$$

${ }^{3}$ Прямой предел $\lim G_{i}$ последовательности $G_{0} \stackrel{f_{0}}{\longrightarrow} G_{1} \stackrel{f_{1}}{\longrightarrow} \cdots$ групп и гомоморфизмов - это группа, элементами которой являются классы эквивалентности нитей, т. е. последовательностей вида $g=\left(g_{k}, g_{k+1}, \ldots\right)$, в которых $g_{i+1}=f_{i}\left(g_{i}\right)$ при каждом $i \geqslant k$. Нити $g$ и $h=\left(h_{l}, h_{l+1}, \ldots\right)$ считаются эквивалентными, если $g_{i}=h_{i}$ для некоторого $i \geqslant \max (k, l)$ (и тем самым также для $i+1, i+2, \ldots)$. Произведение классов $g$ и $h$ есть класс $\left(g_{m} h_{m}, g_{m+1} h_{m+1}, \ldots\right)$, где $m=\max (g, h)$. 
Эта точная последовательность (являющаяся по существу формулой универсальных коэффициентов для точного слева функтора $\underset{\longleftarrow}{\lim }$ ) принадлежит Милнору; по аналогии точной последовательностью Милнора называют и (*).

Понтрягинские когомологии $H^{n}(X)$ определяются как $-(n+1)$-я группа гомологий комплекса $C_{[0, \infty)}^{*}\left(P_{[0, \infty)}\right)=\underset{\lim }{\longrightarrow} C^{*}\left(P_{[0, \infty)}, P_{[i, \infty)}\right)$ коцепей “с $[0, \infty)$ компактными носителями". Двойственным образом к $(* *)$, имеет место естественный изоморфизм

$$
H^{n}(X) \simeq \underline{\lim } H^{n}\left(P_{i}\right)
$$

Вместо того, чтобы приводить доказательство (оно проще проведенного выше доказательства $(*)$ и $(* *)$ и может быть найдено в $\S 4)$, проясним до некоторой степени содержание двойственности. Если $C_{*}-$ цепной комплекс

$$
0 \leftarrow \bigoplus G_{i} \stackrel{\psi}{\longleftarrow} \bigoplus G_{i} \leftarrow 0
$$

где $G_{i}$ - абелевы группы, а $\psi$ переводит всякий $g_{i} \in G_{i}$ в $g_{i}-f_{i}\left(g_{i}\right)$, то, очевидно, $H_{0}\left(C_{*}\right) \simeq \lim G_{i}, H^{0}\left(C_{*}\right) \simeq \lim G_{i}^{\star}$ и $H^{1}\left(C_{*}\right) \simeq \lim ^{1} G_{i}^{\star}$, где $G^{\star}=\operatorname{Hom}(G, \mathbb{Z})$. В этом нет ничего удивительного: как и всякий производный функтор, $\lim ^{1}$ может рассматриваться как топологический объект. Эта точка зрения будет востребована в доказательствах теоремы 3.1 (d) и леммы 3.7 (a).

С. Стинродовские теории против сингулярных. Компакт $X$ удовлетворяет условию $\mathrm{LC}_{\infty}$, если он локально $n$-связен для всех $n$. Для таких компактов сингулярные гомотопии и гомологии совпадают со стинродовскими (см. §6), а сингулярные когомологии - с понтрягинскими (см. [12; гл. $3, \S 1$ и 2 ; 2-е изд., гл. $3, \S 4$ или 1-е изд., добавление 2 редактора рус. пер.]). Для общих компактов, однако, сингулярные группы оказываются уже в некотором роде "аномальными" и трудновычислимыми, тогда как стинродовские по-прежнему обладают многими разумными свойствами, делающими их вычислимыми на практике и применимыми в геометрической топологии. Приведем несколько стандартных иллюстраций этого известного тезиса.

1) В двойственности Александера $H^{n}(X) \simeq \widetilde{H}_{m-n-1}\left(\mathbb{R}^{m} \backslash X\right)$ для компакта $X$ может фигурировать любая разновидность гомологий, но обязаны фигурировать когомологии Понтрягина (а не сингулярные). В двойственности Александера $H_{n}(X) \simeq \widetilde{H}^{m-n-1}\left(\mathbb{R}^{m} \backslash X\right)$ для компакта $X$ может фигурировать любая разновидность когомологий, но обязаны фигурировать гомологии Стинрода (а не сингулярные). См. теорему 4.3. Отметим, что непосредственным следствием любого из этих двух изоморфизмов является теорема Жордана.

2) Конечнократные ${ }^{4}$ накрытия над стинродовски связными (т. е. с тривиальным $\pi_{0}$ ) компактами классифицируются топологизированной стинродовской фундаментальной группой $\pi_{1}$ (см. следствия 7.5 и 7.8). Топологизированной сингулярной фундаментальной группой $\stackrel{\Delta}{1}_{1}$ они не классифицируются (как видно из рассмотрения варшавской окружности, которая как стинродовски, так и линейно связна).

\footnotetext{
${ }^{4}$ и, более общо, произвольные накрытия в категории равномерных (а не топологических) пространств.
} 
3) Группа $[X, K(G, n)]$, определенная либо как группа гомотопических классов отображений $X \rightarrow K(G, n)$, либо как группа стинродовских гомотопических классов (см. предложение 2.3), изоморфна понтрягинским (а не сингулярным) когомологиям $H^{n}(X ; G)$ для всякой счетной абелевой группы $G$ (см. предложение 4.2).

4) Стинродовские гомологии и гомотопии, не говоря уже о понтрягинских когомологиях, обратных пределов допускают разумное описание (см. $(*),(* *)$ и $\left(* *^{\prime}\right)$ выше, а также теоремы 3.1 (с) и 4.1 (iii)), тогда как сингулярные гомологии и гомотопии простейших обратных пределов очень непросто вычислить (см. теорему 1.1 и пример 5.6).

5) Гомологии Стинрода и когомологии Понтрягина удовлетворяют более сильной форме аксиомы вырезания и характеризуются на парах компактов как единственные ординарные теории гомологий и когомологий, удовлетворяющие этой аксиоме "вырезания для отображений” наряду с аксиомой аддитивности [13] (см. точную формулировку ниже в §4).

6) Понтрягинские когомологии совпадают с когомологиями АлександераСпеньера [14] и с пучковыми когомологиями (с постоянными коэффициентами) [12]. Гомологии Стинрода совпадают с гомологиями Масси (двойственными к когомологиям Александера-Спеньера) [15] и, когда коэффициенты конечно порождены ${ }^{5}$, с пучковыми гомологиями Бореля и Мура (с постоянными коэффициентами) [12], [16]. По поводу всех этих утверждений см. [17].

7) $n$-мерный компакт имеет тривиальные гомологии Стинрода (и когомологии Понтрягина) в размерностях $>n$, тогда как сингулярные гомологии ничего не говорят о размерности. Действительно, имеет место следующий пример.

ТЕОРема 1.1 (Баррат-Милнор [18]). Пусть $E^{n}-n$-мерная гавайская серьга, т.е. одноточечная компактификачия $\mathbb{R}^{n} \times \mathbb{N}$. Тогда $(2 n-1)$-мерные сингулярные гомологии $\stackrel{\Delta}{H}_{2 n-1}\left(E^{n}\right)$ нетривиальны.

Оригинальное доказательство изложено на языке теории гомотопий; мы приведем короткое доказательство, основанное на конструкции ПонтрягинаТома и коэффициентах зацепления. Оно также показывает, что $\stackrel{\Delta}{H}_{2 n-1}\left(E^{n} ; \mathbb{Q}\right)$ несчетна. По-видимому, не представляет труда передоказать и полное утверждение результата работы [18] - что $\stackrel{\Delta}{H}_{k n-k+1}\left(E^{n} ; \mathbb{Q}\right)$ несчетна для каждого $k>0$ - таким же образом, используя высшие произведения Масси.

ДокАЗАТЕЛЬство. Пусть $K_{0}, K_{1}, \ldots \subset S^{2 n-1}-$ нуль-последовательность дизъюнктных кусочно линейных экземпляров сферы $S^{n-1}$ таких, что каждая $K_{2 i+1}$ зацеплена с $K_{2 i}$ с коэффициентом зацепления $l_{i}>0$ и не зацеплена со всеми остальными $K_{j}$. Пусть $T_{0}, T_{1}, \ldots$ - дизъюнктные регулярные окрестности сфер $K_{i}$. Определим отображение $f: S^{2 n-1} \rightarrow E^{n}$, проектируя каждую

\footnotetext{
${ }^{5} \mathrm{~B}$ случае, когда коэффициенты не конечно порождены или не локально постоянны, теория Бореля-Мура имеет некоторые известные “дефекты”, подробно обсуждаемые Е. Г. Скляренко в примечаниях редактора русского перевода первого издания книги Г. Э. Бредона [12]. Взамен Скляренко предлагает построить копучковые стинродовские гомологии (не путать с копучковыми чеховскими гомологиями, определенными во втором издании книги Бредона!). Такая теория, в самом деле, может быть построена, если взять за основу второе определение стинродовских гомологий из исходной статьи Стинрода (см. §4); подробности будут опубликованы дополнительно.
} 
$T_{i} \cong S^{n-1} \times \mathbb{R}^{n}$ на $\mathbb{R}^{n} \times\{i\}$ и отправляя дополнение к $\bigcup T_{i}$ на компактифицирующую точку. Мы утверждаем, что $[f] \neq 0 \in \stackrel{\Delta}{H}_{2 n-1}\left(E^{n}\right)$.

Предположим, что $S^{2 n-1}=\partial M$ для некоторого компактного ориентированного $2 n$-псевдомногообразия ${ }^{6} M$ такого, что $f$ продолжается до непрерывного отображения $F: M \rightarrow E^{n}$. Подправим $F$ так, чтобы сделать его симплициальным относительно некоторых триангуляций $[-1,1]^{n} \times \mathbb{N}$ и $F^{-1}\left([-1,1]^{n} \times \mathbb{N}\right)$, где каждая точка $x_{i}=(0, i) \in \mathbb{R}^{n} \times \mathbb{N}$ лежит во внутренности некоторого $n$-симплекса. Тогда каждое $M_{i}:=F^{-1}\left(x_{i}\right)$ есть компактное ориентированное $n$-псевдомногообразие с краем $\partial M_{i}=K_{i}$, регулярная окрестность которого в $M$ гомеоморфна $M_{i} \times I^{n}$, см. [19; теорема 1.3.1]. Более того, $M_{i}$ коориентировано в $M$, как только фиксирована ориентация на $\mathbb{R}^{n} \times \mathbb{N} \subset E$. Пусть $z_{2 i}$ - образующая $H^{n}\left(M, M \backslash M_{2 i}\right) \simeq H^{n}\left(M_{2 i} \times I^{n}, M_{2 i} \times \partial I^{n}\right) \simeq H^{0}\left(M_{2 i}\right)$, соответствующая этой коориентации, и пусть $c_{2 i}$ - ее образ в $H^{n}(M)$. Предположим, что некоторая нетривиальная линейная комбинация $m_{0} c_{0}+\cdots+$ $m_{r} c_{2 r} \in H^{n}(M)$ равна нулю. Пусть $R=M_{0} \cup \cdots \cup M_{2 r}$. Тогда $m_{0} z_{0}+\cdots+$ $m_{r} z_{2 r} \in H^{n}(M, M \backslash R)$ есть образ некоторого $b \in H^{n-1}(M \backslash R)$. Ограничение $b$ на $X:=\partial M \backslash\left(K_{0} \cup \cdots \cup K_{2 r}\right)$ равняется $m_{0} D\left[K_{0}\right]+\cdots+m_{r} D\left[K_{2 r}\right]$, где $D: H_{n-1}\left(K_{0} \sqcup \cdots \sqcup K_{2 r}\right) \rightarrow H^{n-1}(X)$ - двойственность Александера. Следовательно, $b\left(m_{0}\left[K_{1}\right]+\cdots+m_{r}\left[K_{2 r+1}\right]\right)=m_{0}^{2} l_{0}+\cdots+m_{r}^{2} l_{r}>0$. С другой стороны, $\left[K_{2 i+1}\right]=0 \in H_{n-1}(M, M \backslash R)$ для каждого $i$, так как $K_{2 i+1}=\partial M_{2 i+1}$. Полученное противоречие показывает, что все $c_{2 i}$ линейно независимы в $H^{n}(M)$. Значит, $H^{n}(M)$ не является конечно порожденной, что приводит к противоречию.

D. Сравнение с литературой. Следует уточнить (по настоянию рецензента), что в том комбинаторном ракурсе, в котором теория стинродовских гомотопий представлена ниже, она ранее не излагалась в литературе, за исключением отдельных фрагментов. ${ }^{7}$ Зато в литературе нет недостатка в изложениях различных близко родственных построений, в связях которых друг с другом и собственно со стинродовскими гомотопиями поначалу очень легко запутаться; основные из этих связей вкратце подытожены далее. В дальнейшем мы не будем заострять внимание на этих технических тонкостях; в частности, большинство “известных результатов", упоминаемых в настоящей статье, строго говоря, являются лишь переформулировками таковых, причем их эквивалентность исходным формулировкам иногда будет очевидна лишь для эксперта.

Основной объект изучения в настоящей статье - стинродовская гомотопическая категория, в случае компактов определенная в начале $§ 2$. В этом случае она совпадает с сильной шейповой категорией, также известной как тонкая шейповая категория [22]-[24] (см. также [21; §5], [25], [26] и обзор в [27; § III.9]). Более того, в случае компактов первоначальная шейповая категория Борсука (см. [28], [29], [27]) имеет те же классы изоморфизма объектов (см. предложе-

\footnotetext{
${ }^{6}$ Напомним, что [псевдосвязное] (ориентируемое) $m$-nсевдомногообразие с краем $\partial M-$ это пара полиэдров $(M, \partial M)$, допускающая триангуляцию $(K, \partial K)$ такую, что $\left[K \backslash K^{(m-2)}\right] \cup$ $\left[\partial K \backslash(\partial K)^{(m-3)}\right]-\left[\right.$ связное] (ориентируемое) $m$-многообразие с краем $\partial K \backslash(\partial K)^{(m-3)}$.

${ }^{7} \mathrm{~B}$ их число входят доказательство теоремы 2 у Милнора [13], доказательство теоремы 4 у Кодамы [20] и первый абзац доказательства теоремы 4 у Ферри [21].
} 
ние 2.7) и, по крайней мере в конечномерном случае, те же изоморфизмы (см. теорему 3.9); ее морфизмы, вообще говоря, менее информативны (см. примеры в $\S 5)$.

В общем случае сильная шейповая категория имеет в качестве объектов топологические пространства и тем самым отлична от стинродовской гомотопической категории, объектами которой являются полные равномерные пространства. $^{8}$ Последнее вызвано многими причинами; наиболее очевидная состоит в том, что точность гомотопической последовательности расслоения в смысле Серра не выполнена для сильных шейповых групп уже в случае накрытий над компактами. ${ }^{9} \mathrm{C}$ точки зрения результатов $\S 7$, в этом нет ничего удивительного: скорее следовало бы ожидать точность гомотопической последовательности равномерного накрытия - которая и в самом деле имеет место в стинродовских гомотопиях. (Специалист по теории шейпов мог бы возразить, что просто не всякое бесконечнолистное накрытие над компактом следует считать расслоением в смысле сильной теории шейпов; однако для геометрического тополога это и означает, что сильная теория шейпов требует доработки.) Другая важная причина состоит в том, что уже простейшее некомпактное топологическое пространство $[0, \infty)$ не имеет конфинальной последовательности в направленном множестве всех покрытий, и поэтому сильная теория шейпов метризуемых топологических пространств не может обойтись без общих обратных спектров (индексированных направленными множествами, отличными от натуральных чисел). Весьма сложно сделать что-либо геометрическое с телескопами столь общих обратных спектров; к счастью, это и не нужно, поскольку стинродовская гомотопическая категория метризуемых полных равномерных пространств прекрасно обходится обратными последовательностями.

Из-за ограничений по объему и по времени распространение стинродовской гомотопической категории на все сепарабельные метризуемые полные равномерные (SMCU) пространства приходится отложить до отдельной работы, ввиду того, что оно включает в себя решение проблемы Исбелла [11; Research Problem $\left.\mathrm{B}_{2}\right]$, спрашивающей, по существу, можно ли определить бесконечномерные равномерные полиэдры разумным образом. Конечномерно-аппроксимируемые SMCU-пространства, т. е. обратные пределы конечномерных равномерных полиэдров, были хорошо поняты после работ Исбелла; краткий набросок распространения стинродовской гомотопической категории на такие пространства дан в $\S 7$ и применяется к изучению стинродовских гомотопий

\footnotetext{
8Это рассогласуется с терминологией [22] и [30], где термин "стинродовские гомотопии" выступает лишь в качестве еще одного синонима для сильного шейпа.

${ }^{9}$ Пусть $X$ - гавайская улитка из примера 5.7 , т. е. тор автогомеоморфизма $\sigma_{n},(r, i) \mapsto$ $(r, i+1), n$-мерной гавайской серьги $E^{n}=\left(\mathbb{R}^{n} \times \mathbb{Z}\right)^{+}$, где $n \geqslant 2$. Тогда, согласно точной последовательности Милнора $(*)$, группа $\pi_{n+1}(X)$ изоморфна $\mathbb{Z}[\mathbb{Z}]$, как модуль над групповым кольцом стинродовской фундаментальной группы $\pi_{1}(X) \simeq \mathbb{Z}$. Универсальное накрывающее $\widetilde{X}$ (неравномерно) гомеоморфно $E^{n} \times \mathbb{R}$ и тем самым имеет сильный шейп $E^{n}$, для которого $\pi_{n+1}\left(E^{n}\right)=0$. Поскольку $E^{n}$ компактна, $(n+1)$-я сильная шейповая группа $E^{n}$ изоморфна $\pi_{n+1}\left(E^{n}\right)$. Значит, $(n+1)$-я сильная шейповая группа $\widetilde{X}$ тривиальна, поэтому $\widetilde{X} \rightarrow X$ не индуцирует изоморфизма $(n+1)$-х сильных шейповых групп. В то же время, согласно (некомпактному случаю) той же самой точной последовательности $(*),(n+1)$-я стинродовская гомотопическая группа $\pi_{n+1}(\widetilde{X})$ изоморфна $\mathbb{Z}[\mathbb{Z}]$; причем в силу естественности $(*) \pi_{n+1}(\tilde{X}) \rightarrow \pi_{n+1}(X)$ - изоморфизм.
} 
компактов в 8 . Мы могли бы с тем же успехом определить равномерную стинродовскую гомотопическую категорию для таких пространств (ограничение которой на равномерные полиэдры - равномерная гомотопическая категория, а не обычная гомотопическая категория), но нам это не потребуется. В [31] специфическая полуравномерная стинродовская гомотопическая категория была определена для конечномерно-аппроксимируемых SMCU-пространств: за основу берется понятие полуравномерной гомотопии, т. е. (вообще говоря, не равномерно непрерывной) гомотопии в классе равномерно непрерывных отображений. Заметим, что прямая $\mathbb{R}$ полуравномерно стягиваема, но не является равномерно стягиваемой; последнему не стоит удивляться, так как $\mathbb{R}$ не является равномерным абсолютным ретрактом (понятие, определение которого не использует каких-либо гомотопий, см. [11]).

Стинродовские гомотопические и гомологические группы $\pi_{i}(X), H_{i}(X)$ и понтрягинские когомологии $H^{i}(X)$, определенные выше в случае замкнутых подмножеств $X$ евклидова пространства, являются инвариантами стинродовского гомотопического типа (откуда, в частности, следует их корректная определенность как равномерных инвариантов). Группы $H_{i}^{\mathrm{lf}}(X)$ и $H_{\mathrm{c}}^{i}(X)$ - инварианты собственного сильного шейпа (в частности, гомеоморфизма и собственной стинродовской гомотопической эквивалентности).

Стинродовские гомотопические группы компактов были введены Кристи [32] и переоткрыты 30 лет спустя Квигли [33]. В литературе их иногда называют "Quigley approaching groups". Стинродовские гомологии и понтрягинские когомологии компактов были введены самими Стинродом [34] и (в конечномерном случае) Понтрягиным [35]. Понтрягин также рассматривал произвольные замкнутые подмножества $X$ евклидова пространства и определил для них понтрягинские когомологии с компактным носителем $H_{\mathrm{c}}^{i}(X)$ - посредством соотношения $\left(* *^{\prime}\right)$, в котором когомологии полиэдров $P_{i}$ тоже берутся с компактным носителем. Поскольку эти группы являются топологическими инвариантами $X$, в доказательстве корректности определения Понтрягину не пришлось заботиться о том, чтобы окрестности $P_{i}$ выбирались равномерными; однако он был последователен в том, что рассматривал лишь прямые и обратные последовательности (индексированные натуральными числами, а не более общими направленными множествами). Понтрягинские когомологии $H^{i}(X)$ рассматривались в [36] и [37] (см. также [38]) под другими названиями. Что касается стинродовских гомологий $H_{i}(X)$ (и гомотопий $\left.\pi_{i}(X)\right)$, то в некомпактном случае, от обсуждения которого Стинрод в указанной статье предусмотрительно воздержался, они, вероятно, не встречаются в литературе. Локально-конечные стинродовские гомологии $H_{i}^{\mathrm{lf}}(X)$ и понтрягинские когомологии с компактным носителем $H_{\mathrm{c}}^{i}(X)$ рассматривались Скляренко (см. [17]) и другими авторами для произвольных $\sigma$-компактов, т. е. локально-компактных сепарабельных метрических пространств $X$.

Гомологии Понтрягина компактов, как правило, встречаются в литературе под названием "когомологии Чеха". Мы прежде всего преследуем цель избежать путаницы между тремя различными объектами: гомологиями (Александрова-)Чеха(-Даукера), которые не образуют теории гомологий, не удовлетворяя аксиоме точности; когомологиями Чеха(-Даукера), которые образуют тео- 
рию гомологий топологических пространств; и понтрягинскими когомологиями, которые образуют теорию гомологий полных равномерных пространств. В настоящей статье понтрягинские когомологии нам понадобятся только в случае компактов, в котором они совпадают с когомологиями Чеха-Даукера; тем не менее, даже в этом случае название "когомологии Понтрягина" представляется полезным для устранения вводящей в заблуждение (а также исторически неоправданной!) ассоциации с неточными гомологиями Александрова-Чеха. ${ }^{10}$

В литературе получили наибольшее развитие два варианта обобщения гомологий Стинрода и когомологий Понтрягина компактов на некомпактные пространства: во-первых, гомологии Стинрода-Ситникова и уже упомянутые выше когомологии Чеха-Даукера, определенные Ситниковым и Даукером в 1950-х годах с использованием прямого предела (спектра, не обязательно являющегося прямой последовательностью); и, во-вторых, "сильные" (или “когерентные") гомологии и когомологии, определенные Лисицей-Мардешичем и Миминошвили в 1980-х годах с использованием гомотопического обратного предела (спектра, не обязательно являющегося обратной последовательностью). Все эти группы являются инвариантами сильного шейпа, в отличие от $H_{n}$ и $H^{n}$, которые являются лишь инвариантами стинродовского гомотопического типа и тем самым способны улавливать более тонкие феномены (несмотря на то, что их, как правило, проще вычислять). Конкретные примеры откладываются до последующей статьи автора, в которой будут также рассмотрены взаимосвязи между упомянутыми теориями гомологий и когомологий.

\section{E. Содержание параграфов. Дадим краткий обзор статьи.}

$\S 2$. Стинродовская гомотопическая категория. Параграф начинается с основных определений стинродовской теории и обоснования их корректности, принадлежащего Милнору (лемма 2.1). Далее мы доказываем (предложение 2.4) их равносильность первоначальным определениям, предложенным Кристи в 1944 г., с помощью техники (лемма 2.5), которая будет использоваться на протяжении статьи.

10Что касается истории, она выглядит так. В статье 1931 г. в "Mathematische Annalen" [35] Понтрягин ввел прямой предел прямой последовательности групп и использовал его для определения когомологий конечномерного компакта [35; гл. III, § II]. (Он не использовал термин “когомологии", который возник лишь несколькими годами позже.) При этом он рассматривал и обратные последовательности, но смог определить гомологии компакта лишь с рациональными коэффициентами (в приложении II), доказав попутно, что всякая обратная последовательность конечномерных векторных пространств над $\mathbb{Q}$ эквивалентна обратной последовательности эпиморфизмов. По всей видимости, Чех не был знаком с этой статьей Понтрягина на тот момент, когда он писал статью [39], вышедшую в 1932 г. - свою самую раннюю работу на данную тему. Однако в ней он и не пытался рассматривать когомологии или прямые пределы, но ввел обратный предел обратного спектра групп и использовал его, чтобы прояснить смысл определения гомологий компакта, данного Александровым (1929), и распространить его на неметризуемые компактные пространства. (Независимые эквивалентные определения гомологий метрических компактов были также даны Вьеторисом (1927) и в конечномерном случае Лефшецем (1930); впрочем, эквивалентность подходов Александрова и Лефшеца с подходом Вьеториса была установлена намного позже [40].) В той же статье Чех также попытался определить гомологии некомпактных пространств, но вместо этого определил нечто, что в локально-компактном случае оказывается гомологиями Александрова-Чеха компактификации Стоуна-Чеха исходного пространства (ср. [41; гл. Х, теорема 9.12]) - которые не являются гомотопическим инвариантом исходного пространства. Лишь в 1950 г. Даукер "исправил" это определение Чеха. 
Дальнейшие результаты $\S 2$ связывает рассмотрение частных ситуаций, позволяющих по-разному взглянуть на стинродовские гомотопии в целом. Вкратце: стинродовские гомотопии и про-гомотопии (предложения 2.6, 2.8 (b)); пунктированные и непунктированные стинродовские гомотопии (предложение 2.9); стинродовски связные и линейно связные компакты (пример 2.10); чеховское распространение функторов (предложение 2.3); клеточноподобные компакты (предложение 2.7). В целом, задача $\S 2$ - изложить новый простой подход к основаниям теории; мы также приводим простое доказательство принадлежащей Дыдаку и Сигалу “теоремы Фокса" для стинродовских гомотопий (предложение $2.8(\mathrm{a}))$.

$\S 3$. Группы гомотопий. Определены стинродовские гомотопические группы, изложены простейшие методы работы с ними (теорема 3.1, пример 3.2 и леммы $3.3,3.4$ ), проиллюстрированные на $\mathrm{UV}_{n}$-компактах (предложение 3.5 ). Свойство “уточненной непрерывности" 11 стинродовских гомотопических групп (теорема 3.1 (с)) автору не удалось отыскать в литературе. Основной результат 33 - "теорема Уайтхеда" для стинродовских гомотопий (теорема 3.6), отвечающая на вопрос А. Коямы 1983 г. Даны также два простых геометрических доказательства теоремы стабильности Эдвардса-Гэгана (теорема 3.10) и простое доказательство теоремы Дыдака и Кодамы о стабильности объединения (следствие 3.14).

Наконец, определяются стинродовские расслоения, которые оказываются упрощенной, но равносильной версией шейповых расслоений Мардешича и Рашинга. Это позволяет упростить доказательства Кэйти точной последовательности в стинродовских гомотопиях (теорема $3.15(\mathrm{~b})$ ) и стинродовской гомотопической эквивалентности слоев (теорема $3.15(\mathrm{c})$ ) для стинродовских расслоений, а также доказательство Мардешича и Рашинга того, что клеточноподобные отображения между конечномерными компактами являются стинродовскими расслоениями (теорема $3.15(\mathrm{~d})$ ). Также показано, что стинродовское расслоение над связным компактом индуцирует сюръекцию на $\pi_{0}$ (теорема $3.15\left(\mathrm{~b}^{\prime}\right)$ ).

$\S 4$. Гомологии и когомологии. Кратко напоминаются определение и основные свойства групп гомологий Стинрода и когомологий Понтрягина (теорема 4.1, предложение 4.2). В качестве иллюстрации приводится элементарное доказательство теорем двойственности Александера (теорема 4.3). Дано аккуратное доказательство (абсолютной) “теоремы Гуревича" для стинродовских гомотопий (теорема 4.4) вместе с контрпримером к "доказательству", доступному ранее.

$\S 5$. Некоторые примеры. За некоторыми исключениями, этот и последующие параграфы предполагают известными лишь утверждения 2.1-2.5, 3.1-3.4, 4.1-4.2 и независимы друг от друга. Параграф 5 - небольшой зверинец компактов, иллюстрирующих различные особенности стинродовских гомотопий и гомологий в сравнении с сингулярными теориями. В их число входят соленоид (пример 5.3), гавайская серьга (пример 5.6) и альтернативное воплощение примера Дыдака и Здравковской (пример 5.8).

\footnotetext{
${ }^{11}$ ср. параграф “Непрерывность против точности” в книге Стинрода и Эйленберга [41].
} 
§6. Сравнение теорий. Этот параграф посвящен сравнению сингулярных, стинродовских и чеховских теорий в предположении различных условий локальной связности, а также сравнению самих этих условий. Основной результат $\S 6$ - гомотопическая теорема реализации (теорема 6.5). Также доказана соответствующая гомологическая $\mathrm{LC}_{n-1}$-теорема реализации (теорема 6.7), анонсированная также Щепиным (неопубликовано) и влекущая результат Эды и Кавамуры 1999 г. Далее приводятся геометрические доказательства теорем сравнения Мардешича (теорема $6.8(\mathrm{~d}))$ и Юссилы (теорема $6.8(\mathrm{~b}))$ и доказываются еще два неопубликованных результата Щепина: реализация стинродовских циклов фрактальными псевдомногообразиями для компактов, удовлетворяющих $\mathrm{HLC}_{n-1}$ (следствие 6.10) и совпадение условия $\mathrm{HLC}_{n}$ с условием $\mathrm{HLC}_{n}$ (теорема $6.8(\mathrm{a})$ ). На пути к этим результатам мы передоказываем гомотопическую теорему сравнения Гуревича, Борсука и др. (теорема 6.1) и принадлежащую Ферри “теорему Гуревича в конечном счете" (лемма 6.3).

В качестве применения предложено геометрическое "объяснение" конечной порожденности стинродовских гомологий $\mathrm{HLC}_{\infty}$-компактов (теорема 6.11).

$\S 7$. Теория накрытий. Дано упрощение формулировки и доказательства теоремы Фокса о классификации наложений (теорема 7.4). Подмечено, что наложения эквивалентны равномерным накрытиям (теорема 7.6). Понятие универсального обобщенного наложения иллюстрируется на примере "ноттингемского компакта" (пример 7.12).

§8. Нульмерные гомотопии. Этот параграф посвящен стинродовски связным (pointed 1-movable) компактам. Получено простое доказательство теоремы Гэгана-Красинкевича о том, что виртуально несвязные по Стинроду компакты имеют пустые стинродовские компоненты связности (теорема 8.4). Контрпример Брина (по-видимому, неопубликованный) к обращению этой теоремы по-видимому восстановлен (пример 8.6). Далее, дается относительно простое доказательство теоремы Красинкевича-Минца (теорема 8.7), из которой следует, в частности, стинродовская связность линейно связных компактов. В качестве применения получена характеризация стинродовски связных компактов в терминах количества компонент равномерной связности их равномерных накрывающих пространств (теорема 8.9). Также приводятся упрощенные доказательства теоремы Макмиллана-Красинкевича о непрерывных образах (теорема 8.2) и теоремы Красинкевича об объединении (теорема 8.3) стинродовски связных компактов.

Алгебраико-топологическое содержание статъи. Получена "классификация" счетных про-групп типа $\omega$ в терминах их $\lim _{\longleftarrow} \lim ^{1}$ (теорема 3.8), что

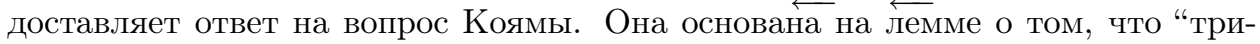
виальность $\lim ^{1}$ влечет условие Миттаг-Леффлера для счетных про-множеств смежных классов" (лемма 3.7 (b)). В статью включены простые изложения известных результатов о $\lim ^{1}$ и об условии Миттаг-Леффлера (утверждения (b) и (d) теоремы 3.1, пример 3.2, леммы 3.3, 3.4, 3.7 (a), 3.11). Приводится также простое геометрическое построение длинной точной последовательности Дж. Г.К. Уайтхеда, в которую включаются гомоморфизмы Гуревича разных размерностей (лемма 4.5). 
Общетопологическое содержание статъи. Вводится понятие сходимости обратной последовательности метризуемых равномерных пространств, обобщающее условие Миттаг-Леффлера для обратных последовательностей множеств (снабжаемых дискретной равномерностью) и предоставляющее "объяснение" бурбаковской “теоремы Миттаг-Леффлера" (следствие 7.10) и тем самым также теоремы Бэра о категории (следствие 7.11).

Благодарности. Эта работа никогда не появилась бы без (i) разговора с Е. Дыдаком, который застиг меня врасплох вопросом о том, задумывался ли я всерьез над проблемой Гильберта-Смита; (ii) 70-летия С. П. Новикова, по причине которого я стал писать 5-страничную заметку на тему проблемы Гильберта-Смита, предназначенную для юбилейного тома; (iii) переписки c А. Н. Дранишниковым, которая убедила меня в том, что я должен прекратить думать над проблемой Гильберта-Смита до тех пор, пока не стану лучше понимать стинродовские гомотопии; (iv) предусмотрительности редакторов упомянутого тома: В. М. Бухштабера, О.К. Шейнмана и Е. В. Щепина - которые путем продуманного подбора сдвигов последних сроков добились загадочного преобразования упомянутой заметки в данную статью.

Я благодарен всем топологам Университета Теннесси за гостеприимство и поддержку в течение моего визита и сверх того Ежи Дыдаку за многочисленные полезные замечания и ссылки на литературу, Коле Бродскому, Пепе (Хосе) Ихесу, Брендону Лабузу и Конраду Плауту за полезные обсуждения и замечания о равномерных пространствах и равномерных накрытиях. Обсуждения с П. М. Ахметьевым, А. Н. Дранишниковым, Р. В. Михайловым, Ю. Б. Рудяком, Ю. Турыгиным, А.В. Чернавским и Е.В. Щепиным, имевшие место на протяжении разных лет, помогли мне лучше понять стинродовские гомологии. Я особенно признателен Мише Скопенкову за внимание к моим рассуждениям, помогшее вовремя обнаружить две ошибки, и за замечания к тексту статьи. Также я глубоко благодарен Н. Мазуренко, которая помогла с переводом англоязычного оригинала работы на русский.

\section{§ 2. Стинродовская гомотопическая категория}

Пусть $X$ - компакт. Тогда $X$ - предел обратной последовательности $P=$ $\left(\cdots \stackrel{p_{1}}{\longrightarrow} P_{1} \stackrel{p_{0}}{\longrightarrow} P_{0}\right)$ компактных полиэдров и кусочно линейных отображений (Х. Фройденталь, 1937; короткое и ясное изложение доказательства можно найти в $[11 ; \mathrm{V} .33])$. Teлескоп $P_{[0, \infty)}$ - это бесконечное объединение

$$
\cdots \cup_{P_{2}} M C\left(p_{2}\right) \cup_{P_{1}} M C\left(p_{1}\right)
$$

цилиндров связующих отображений. Он снабжен проекцией $\pi_{P}: P_{[0, \infty)} \rightarrow$ $[0, \infty)$, отправляющей каждый $M C\left(p_{i}\right)$ на $[i, i+1]$ с сохранением уровней. Для каждого $J \subset[0, \infty)$ обозначим $\pi_{P}^{-1}(J)$ через $P_{J}$. Мы будем предполагать, что $P_{0}$ - точка; это не ограничивает общности, поскольку мы всегда можем дополнить заданную обратную последовательность отображением $P_{0} \rightarrow \mathrm{pt}$ и сдвинуть индексы на единицу (эти операции не изменяют обратного предела). Так всякий $P_{[0, k]}$ становится стягиваемым. 
Предел $P_{[0, \infty]}$ обратной последовательности ретракций $r_{k}: P_{[0, k+1]} \rightarrow P_{[0, k]}$, легко видеть, является компактификацией $P_{[0, \infty)}$ посредством $X$. Проекции $\Pi_{k}: P_{[0, \infty]} \rightarrow P_{[0, k]}$ компонируются с гомотопиями $r_{k} \simeq \operatorname{id}_{P_{[0, k+1]}}$ в гомотопию $\Pi_{t}: P_{[0, \infty]} \rightarrow P_{[0, \infty]}, t \in[0, \infty]$, где $\Pi_{\infty}-$ тождественное отображение. Компакт $P_{[0, \infty]}$ удовлетворяет условию $\mathrm{LC}_{\infty}$, так как он $\varepsilon$-деформационно ретрагируется (посредством $\Pi_{1 / \varepsilon}$ ) на компактный полиэдр для каждого $\varepsilon>0 .{ }^{12}$

Пусть $Y$ - другой компакт, и пусть $Q=\left(\cdots \stackrel{q_{1}^{2}}{\longrightarrow} Q_{1} \stackrel{q_{0}^{1}}{\longrightarrow} Q_{0}\right)$ - обратная последовательность компактных полиэдров и кусочно линейных отображений $\left(Q_{0}=\mathrm{pt}\right)$ с обратным пределом $Y$.

Лемма 2.1. (а) (Милнор) Всякое отображение $f: X \rightarrow Y$ продолжсается до непрерьвного отображения $f_{[0, \infty]}: P_{[0, \infty]} \rightarrow Q_{[0, \infty]}$, которое

(i) совпадает $\mathrm{c} f \mathrm{на} X$ u

(ii) сужается до собственного отображсения $f_{[0, \infty)}: P_{[0, \infty)} \rightarrow Q_{[0, \infty)}$.

(b) Всякие два отображения, удовлетворяющие (i) u (ii), гомотопны в классе отображений, удовлетворяющих (i) и (ii).

Часть (а) - это, по существу, теорема 2 из работы Милнора [13]. Пока статья Милнора оставалась неопубликованной, $\operatorname{ANR} P_{[0, \infty]}$ и лемма 2.1 были переоткрыты в середине 1970-х в [42]-[44] и соответственно [20], [45] (см. также $\left[23\right.$; лемма 3.4]). "Дискретный телескоп" $P_{\mathbb{N} \cup \infty} \subset P_{[0, \infty]}$ и бесконечный телескоп $P_{[0, \infty)}$ были рассмотрены уже Х. Фройденталем (ср. [43]) и С. Лефшецем (ср. [13]) в 1930-х годах. Нижеприведенное доказательство леммы 2.1 упрощает подход Красинкевича [43] .

ДоказАтельство. (а) Триангулируем $P_{[0, \infty)}$ так, чтобы диаметры всех симплексов стремились к нулю при приближении к $X$, и пусть $r: X \cup P_{[0, \infty)}^{(0)} \rightarrow X-$ ретракция, где $P_{[0, \infty)}^{(0)}$ обозначает 0-мерный остов триангуляции. Пусть $r_{J}-$ ограничение $r$ на $P_{J}^{(0)}$. Поскольку $q_{i}^{\infty}, q_{i}^{i+1}$ и $r$ равномерно непрерывны, для каждого $i$ существует $j=j(i)$ такое, что композиция

$$
P_{[j(i), \infty)}^{(0)} \stackrel{r_{[j(i), \infty)}}{\longrightarrow} X \stackrel{q_{i}^{\infty}}{\longrightarrow} Q_{i}
$$

продолжается "по линейности" до отображения $\varphi_{i}: P_{[j(i), \infty)} \rightarrow Q_{i}$, причем композиция

$$
P_{[j(i+1), \infty)} \stackrel{\varphi_{i+1}}{\longrightarrow} Q_{i+1} \stackrel{q_{i}^{i+1}}{\longrightarrow} Q_{i}
$$

гомотопна "по линейности" ограничению отображения $\varphi_{i}$.

Комбинируя подходящие ограничения этих отображений и гомотопий, мы получим продолжение отображения $f$ до непрерывного $F: P_{[k, \infty]} \rightarrow Q_{[0, \infty]}$, где $k=j(1)$, причем $F\left(P_{[k, \infty)}\right) \subset Q_{[0, \infty)}$ по построению. Компактное множество $F\left(P_{k}\right)$ лежит в некотором $Q_{[0, l]}$, которое является стягиваемым. Следовательно, $F$ продолжается до отображения $f_{[0, \infty]}: P_{[0, \infty]} \rightarrow Q_{[0, \infty]}$, удовлетворяющего (i) и (ii).

\footnotetext{
${ }^{12}$ На самом деле, хорошо известно (но не требуется для наших целей), что $P_{[0, \infty]}-\mathrm{ANR}$ (и, следовательно, AR). Это следует из любой из двух известных характеризаций ANR-ов: Лефшеца и Ханнера (приведенных соответственно в “Теориях ретрактов” Борсука и Ху).
} 
(b) Поскольку $P_{[0, \infty]}$ линейно связен и локально линейно связен, ретракция $r$ из доказательства пункта (а) гомотопна тождественному отображению неподвижно на $X$. Так как $\left(P_{[0, \infty]}, X \cup P_{[0, \infty)}^{(0)}\right)$ - пара Борсука, то $r$ продолжается до непрерывного отображения $R: P_{[0, \infty]} \rightarrow P_{[0, \infty]}$, гомотопного тождественному отображению неподвижно на $X$. Тогда заданные отображения $F, G: P_{[0, \infty]} \rightarrow Q_{[0, \infty]}$ гомотопны отображениям $F R$ и $G R$ неподвижно на $X$. Для всякой возрастающей последовательности $k_{1}, k_{2}, \ldots$ телескопическое связующее отображение $\left[q_{0}^{k_{0}}, q_{\infty}^{k_{\infty}}\right]: Q_{\left[k_{0}, \infty\right]} \rightarrow Q_{[0, \infty]}$ гомотопно тождественному отображению неподвижно на $X$. Наконец, $\left[q_{0}^{k_{0}}, q_{\infty}^{k_{\infty}}\right] F R$ гомотопно неподвижно на $X$ отображению $\left[q_{0}^{k_{0}}, q_{\infty}^{k_{\infty}}\right] G R$ для подходящей последовательности $\left(k_{i}\right)$ по доказательству части (a), примененной к $f \times \operatorname{id}_{I}: X \times I \rightarrow Y$.

Следствие 2.2. При $Y=X$ отображение $\left(\mathrm{id}_{X}\right)_{[0, \infty)}: P_{[0, \infty)} \rightarrow Q_{[0, \infty)}-$ собственная гомотопическая эквивалентность.

Стинродовская гомотопическая категория. Мы определяем стинродовский гомотопический класс $X \rightsquigarrow Y$ как собственный гомотопический класс собственного отображения $f: P_{[0, \infty)} \rightarrow Q_{[0, \infty)}$. Благодаря собственным гомотопическим эквивалентностям $\left(\operatorname{id}_{X}\right)_{[0, \infty)}$ и $\left(\operatorname{id}_{Y}\right)_{[0, \infty)}$ это определение не зависит от выбора обратных последовательностей $P$ и $Q$. Композиция $X \rightsquigarrow Y \rightsquigarrow Z$ определяется с помощью взятия композиции подходящих собственных отображений. Относя отображению $f: X \rightarrow Y$ собственный гомотопический класс отображения $f_{[0, \infty)}$, обозначаемый $[f]$, мы тем самым получаем отображение $\stackrel{\Delta}{\tau}$ из множества $[X, Y]^{\triangle}$ гомотопических классов отображений $X \rightarrow Y$ в множество $[X, Y]$ стинродовских гомотопических классов $X \rightsquigarrow Y$, которое естественно по обеим переменным. Стинродовский гомотопический класс $f: X \rightsquigarrow Y$ называется стинродовской гомотопической эквивалентностъю, если существует $g: Y \rightsquigarrow X$ такой, что $g f=\left[\mathrm{id}_{X}\right]$ и $f g=\left[\mathrm{id}_{Y}\right]$, другими словами, если $f$ представляется собственной гомотопической эквивалентностью. Когда такой $f$ существует, говорят, что $X$ и $Y$ - одного шейna (ср. англ. shape: $\underline{\text { Steenrod }}$ homotopy type).

Стинродовские гомотопические классы были введены (под другим названием) Д. Кристи в диссертации 1944 г. [32]. Он дал несколько эквивалентных определений и нашел несчетное множество стинродовских гомотопических классов из точки в двуадический соленоид (относительно них см. пример 5.3). Предложенные Кристи варианты определения стинродовского гомотопического класса (см. предложение 2.4 (а) ниже), так же как и версия Ферри [21; §5] (см. предложение 2.4 (b)), немного проще тем, что частично обходят лемму 2.1, но, с другой стороны, их асимметрия не позволяет определить композицию стинродовских гомотопических классов. По-видимому, сам Кристи не был знаком со статьей Стинрода 1940 г. [34], где были введены стинродовские гомологии (и, кроме прочего, было найдено несчетное множество 0-мерных стинродовских гомологических классов двуадического соленоида). Однако статья Стинрода фигурирует в списке литературы изданной в 1942 г. книги [40] Лефшеца - научного руководителя диссертации Кристи.

В нескольких случаях (в теореме 3.10, предложении 4.2, теореме 7.4) нам понадобятся стинродовские гомотопические классы из компакта в не обязательно 
компактный полиэдр, такой как классифицирующее пространство ${ }^{13}$ счетной группы. Пусть $P=\left(\cdots \rightarrow P_{1} \rightarrow P_{0}\right)$ и $Q=\left(\cdots \rightarrow Q_{1} \rightarrow Q_{0}\right)$ - обратные последовательности (быть может, некомпактных) полиэдров. Тогда отображение $Q_{[0, \infty)} \rightarrow R_{[0, \infty)}$ между двумя бесконечными телескопами называется полусобственным, если для любого $k$ найдется $l$ такое, что $f^{-1}\left(R_{[0, k]}\right) \subset Q_{[0, l]}$. Если теперь все $P_{i}$ компактны и $X=\lim P_{i}$, а $Q_{0}=$ pt и $Q_{i}=K$ для всех $i>0$, где $K$ - некомпактный полиэдр, то мы определяем стинродовский гомотопический класс $X \rightsquigarrow K$ как полусобственный гомотопический класс полусобственного отображения $f: P_{[0, \infty)} \rightarrow Q_{[0, \infty)}$. Другое, но эквивалентное определение будет дано в $\S 7$ в случае, когда $K$ конечномерен. Через $[X, K]$ обозначим множество стинродовских гомотопических классов $X \rightsquigarrow K$. Лемма 2.1 доставляет отображение $\stackrel{\Delta}{\tau}:[X, K]^{\triangle} \rightarrow[X, K]$.

ПреДлОЖеНИе 2.3. (а) Если $K-$ nолиэдp, mo $\stackrel{\Delta}{\tau}:[X, K]^{\triangle} \rightarrow[X, K]-б u$ екция.

(b) Если $K-$ полиэдр и $X$ - предел обратной последовательности полиэдров $P_{i}$, то существует естественная биекция $[X, K] \rightarrow \underset{\lim }{\longrightarrow}\left[P_{i}, K\right]$.

Часть (а) содержится, например, в [23; теорема 4.4]; часть (b) тоже хорошо известна.

Доказательство. (а) Так как $K$ - полиэдр, то в качестве $Q_{[0, \infty)}$ можно взять открытый конус $C K \cup_{K=K \times 0} K \times[0, \infty)$. Каждое полусобственное отображение $P_{[0, \infty)}$ в этот открытый конус сужается до отображения $P_{[k, \infty)} \rightarrow K \times$ $[0, \infty) \simeq K$ для некоторого $k$. С другой стороны, каждое $f: P_{[k, \infty)} \rightarrow K$ гомотопно $f^{\prime}$, удовлетворяющему $\left.f^{\prime}\right|_{P_{i+1}}=\left(\left.f^{\prime}\right|_{P_{i}}\right) p_{i}$ для каждого $i$ и, следовательно, продолжающемуся по непрерывности на $X$. Повторяя ту же конструкцию для гомотопий, получаем композицию $[X, K] \stackrel{\varphi}{\longrightarrow} \underset{\lim }{\longrightarrow} S_{k} \stackrel{\psi}{\longrightarrow} \underset{\longrightarrow}{\lim }\left[P_{[k, \infty)}, K\right] \stackrel{\chi}{\longrightarrow}[X, K]^{\triangle}$, где $S_{k}$ - множество полусобственных гомотопических классов полусобственных отображений $P_{[k, \infty)} \rightarrow K \times[0, \infty)$. С другой стороны, $\stackrel{\Delta}{\tau}$ раскладывается в композицию $[X, K]^{\triangle} \stackrel{\chi^{\prime}}{\longrightarrow} \underset{\lim }{\longrightarrow}\left[P_{[k, \infty)}, K \times[0, \infty]\right] \stackrel{\psi^{\prime}}{\longrightarrow} \underset{\longrightarrow}{\lim } S_{k} \stackrel{\varphi^{\prime}}{\longrightarrow}[X, K]$ по построению. Легко проверить, что $\varphi^{\prime}=\varphi^{-1}, \psi^{\prime}=\psi^{-1}$ и $\chi^{\prime}=\chi^{-1}$. В частности, чтобы убедиться, что $\chi^{\prime} \chi=\mathrm{id}$, заметим, что если два отображения $P_{[k, \infty]} \rightarrow K$ совпадают на $X$, то их ограничения на некоторый $P_{[l, \infty]}$ гомотопны.

(b) Каждый $P_{i}$ есть деформационный ретракт $P_{[i, \infty)}$, поэтому $\underset{\lim }{\longrightarrow}\left[P_{i}, K\right]=$ $\underset{\lim }{\longrightarrow}\left[P_{[i, \infty)}, K\right]$. Последний совпадает с $[X, K]$ по доказательству пункта (а).

Предложение 2.3 доказано.

Вернемся к рассмотрению стинродовских гомотопических классов из компакта в компакт.

ПреДЛОЖЕНИЕ 2.4. Пусть $Y$ - обратный предел обратной последовательности компактных полиэдров $Q_{i}$, причем $Q_{0}$ не предполагается точкой. Стинродовские гомотопические классы $X \rightsquigarrow Y$ находятся в биекиии с:

13 Здесь уместно напомнить, что всякий CW-комплекс гомотопически эквивалентен локально конечному полиэдру. Например, гомотопический тип $K(\mathbb{Z} / 2,1)$ представляется прямым телескопом цепочки включений $\mathbb{R} P^{1} \subset \mathbb{R} P^{2} \subset \cdots$. 
(а) классами сохраняющих уровни отображений $X \times[0, \infty) \rightarrow Q_{[0, \infty)}$ относительно сохраняющей уровни гомотопии;

(b) классами собственных отображений $X \times[0, \infty) \rightarrow Q_{[0, \infty)}$ относительно собственной гомотопии.

Заметим, что (наиболее полезный) случай, когда $X$ является полиэдром, очевиден (если не принимать в расчет использование леммы $2.5(\mathrm{a}))$, так как в качестве $P_{[0, \infty)}$ можно взять открытый конус $C X \cup_{X=X \times\{0\}} X \times[0, \infty)$, а $C X$ можно отбросить заодно с $Q_{[-1,0]}:=C Q_{0}$.

Утверждение части (b) неявно присутствует в определениях [21; §5], а доказательство, быть может, можно извлечь из [23] (см. там, в частности, леммy 3.4).

ДоКАЗАТЕЛЬСТво. Если $Q_{0}=\mathrm{pt}$, то стинродовский гомотопический класс незамедлительно доставляет собственный гомотопический класс как в (b), а по нижеприведенной лемме 2.5 (a) собственный гомотопический класс как в (b) доставляет сохраняющий уровни гомотопический класс как в (а). Общий случай сводится к случаю $Q_{0}=$ pt аналогично лемме 2.5 (a).

Пусть $f_{[0, \infty)}: X \times[0, \infty) \rightarrow Q_{[0, \infty)}$ сохраняет уровни. Из леммы 2.1 (а) следует, что каждое $f_{i}: X \rightarrow Q_{i}$ пропускается через отображение $F_{i}: P_{\left[k_{i}, \infty\right]} \rightarrow Q_{i}$ для некоторого $k_{i}$. Из леммы $2.1(\mathrm{~b})$ следует, что композиция $P_{\left[k_{i+1}, \infty\right]} \stackrel{F_{i+1}}{\longrightarrow}$ $Q_{i+1} \stackrel{q_{i}^{i+1}}{\longrightarrow} Q_{i}$, ограниченная на $P_{\left[l_{i}, \infty\right]}$ для некоторого $l_{i} \leqslant \min \left(k_{i}, k_{i+1}\right)$, гомотопна ограничению $F_{i}$. Не теряя общности, можно считать, что последовательность $l=\left(l_{1}, l_{2}, \ldots\right)$ неубывающая. Тогда $f_{[0, \infty)}$ пропускается через сохраняющее уровни отображение $R_{[0, \infty)} \rightarrow Q_{[0, \infty)}$, где $R_{i}=P_{\left[l_{i}, \infty\right]}$, а связующие отображения $R_{i+1} \rightarrow R_{i}$ суть включения. Тогда $f_{[0, \infty)}$ также пропускается через сохраняющее уровни отображение $f_{[0, \infty)}^{\prime}: P_{[0, \infty)}^{l} \rightarrow Q_{[0, \infty)}$, где $P_{i}^{l}=P_{l_{i}}$.

Аналогичное рассуждение показывает, что если $f_{[0, \infty)}$ гомотопно с сохранением уровней отображению $g_{[0, \infty)}$, то $f_{[0, \infty)}^{\prime}$ и аналогичным образом построенное $g^{\prime}: P_{[0, \infty)}^{m} \rightarrow Q_{[0, \infty)}$ будут гомотопны с сохранением уровней, если их предварить сохраняющими уровни отображениями $P_{[0, \infty)}^{n} \rightarrow P_{[0, \infty)}^{l}$ и $P_{[0, \infty)}^{n} \rightarrow P_{[0, \infty)}^{m}$ для некоторой последовательности $n=\left(n_{i}\right)$, мажорирующей обе последовательности $l$ и $m$.

Лемма 2.5. (а) Каждое собственное отображение $X \times[0, \infty) \rightarrow Q_{[0, \infty)}$ собственно гомотопно сохраняющему уровни отображению. Собственно гомотопные сохраняющие уровни отображения $X \times[0, \infty) \rightarrow Q_{[0, \infty)}$ гомотопны с сохранением уровней.

$\left(\mathrm{b}_{0}\right)$ Каждое собственное отображение $f: P_{[0, \infty)} \rightarrow Q_{[0, \infty)}$ собственно гомотопно такому $f^{\prime}$, что для некоторой бесконечной подпоследовательности $P_{i}^{k}=P_{k_{i}}$ композиция "переиндексирующей" собственной гомотопической эквивалентности $k_{[0, \infty)}: P_{[0, \infty)}^{k} \rightarrow P_{[0, \infty)}$ u $f^{\prime}$ сохраняет уровни.

$\left(\mathrm{b}_{1}\right)$ Если сохраняющие уровни отображения $f, g: P_{[0, \infty)} \rightarrow Q_{[0, \infty)}$ собственно гомотопны, то существует бесконечная возрастающая последовательность $k=\left(k_{i}\right)$ такал, что композичии отображсений $f^{k}, g^{k}: P_{[0, \infty)}^{k} \rightarrow Q_{[0, \infty)}^{k}$ с сохраняющим уровни отображением $\left[p_{0}^{k_{0}}, p_{\infty}^{k_{\infty}}\right): Q_{[0, \infty)}^{k} \rightarrow Q_{[0, \infty)}$ гомотопны с сохранением уровней. 
Части $\left(\mathrm{b}_{0}\right)$ и $\left(\mathrm{b}_{1}\right)$, судя по всему, близки по духу к [22; следствие (3.7.20)].

Доказательство. (а) Если отображение $\Phi: X \times[0, \infty) \rightarrow Q_{[0, \infty)}-$ собственное, то $\varphi:[0, \infty) \rightarrow[0, \infty)$, определенное формулой $\varphi(t)=\inf (p(\Phi(X \times[t, \infty))))$, является собственной неубывающей непрерывной функцией и, следовательно, может быть аппроксимировано гомеоморфизмом $\tilde{\varphi}$ таким, что $\tilde{\varphi}(t) \leqslant \varphi(t)$ для каждого $t$. Тогда $\Phi$ собственно гомотопно $\Phi^{\prime}$, определенному формулой $\Phi^{\prime}(x, t)=\Pi_{\tilde{\varphi}(t)}(\Phi(x, t))$. Наконец, $\Phi^{\prime}$ гомотопно $\Phi^{\prime \prime}$, определенному формулой $\Phi^{\prime \prime}(x, t)=\Phi^{\prime}\left(x, \tilde{\varphi}^{-1}(t)\right)$, которое сохраняет уровни.

Для доказательства второго утверждения достаточно отметить, что гомотопию от $\Phi$ к $\Phi^{\prime \prime}$ можно считать непрерывно зависящей от $\Phi$ и тождественной всякий раз, когда $\Phi$ сохраняет уровни.

$\left(\mathrm{b}_{0}\right)$ и $\left(\mathrm{b}_{1}\right)$. Доказывается аналогично (a). Собственное отображение $k_{[0, \infty)}$ заменяет переход от $\Phi^{\prime}$ до $\Phi^{\prime \prime}$, а при переходе от $\Phi$ к $\Phi^{\prime}$ возникает сохраняющее уровни отображение $\left[p_{0}^{k_{0}}, p_{\infty}^{k_{\infty}}\right)$.

ПреДЛОЖЕНИЕ 2.6. Пусть $X$ и $Y$ - пределы обратных последовательностей компактных полиэдров $P=\left(\cdots \stackrel{p_{1}}{\longrightarrow} P_{1} \stackrel{p_{0}}{\longrightarrow} P_{0}\right)$ и $Q=\left(\cdots \stackrel{p_{1}}{\longrightarrow} P_{1} \stackrel{p_{0}}{\longrightarrow} P_{0}\right)$. Следующие утверждения равносильны:

(i) $X$ и Y имеют один и тот же шейп;

(ii) $P \simeq Q$ в про-гомотопиях, т.е. существуют возрастающая последовательность $k: \mathbb{N} \rightarrow \mathbb{N}$ и сохраняющие уровни отображсения $f_{[0, \infty)}: P_{[0, \infty)}^{k} \rightarrow$ $Q_{[0, \infty)}^{k}$ u $g_{[0, \infty)}: Q_{[0, \infty)}^{k s} \rightarrow P_{[0, \infty)}^{k}$, где $P_{i}^{k}$ обозначает $P_{k(i)}$ u $s(i)=i+1$, maкие, что $g_{i} f_{i+1}: P_{k(i+1)} \rightarrow P_{k(i)}$ и $f_{i} g_{i}: Q_{k(i+1)} \rightarrow Q_{k(i)}$ гомотопны связующим отображсения для всех $i$;

(iii) $P$ и $Q$ эквивалентны относительно отношения эквивалентности, порожденного переходом $\kappa$ подпоследовательностям и гомотопированием связующих отображений.

(iv) $P_{[0, \infty)} u Q_{[0, \infty)}$ имеют один $и$ тот же собственный простой гомотопический тuп.

Доказательство импликации (ii) $\Rightarrow$ (i) восходит к [22; теорема (5.2.9)]. Отношение эквивалентности в (iii) было введено Зибенманом [46].

ДокАЗАтельство. (iii) $\Rightarrow$ (iv) не вызывает затруднений (см. подробности в [46]), a (iv) $\Rightarrow$ (i) непосредственно вытекает из определений. (i) $\Rightarrow$ (ii) получается с помощью леммы $2.5\left(\mathrm{~b}_{0}\right),\left(\mathrm{b}_{1}\right)$. Наконец, предполагая (ii), $P$ и $Q$ эквивалентны в смысле (iii) комбинированной обратной последовательности $\cdots \stackrel{g_{1}}{\longrightarrow} P_{k(1)} \stackrel{f_{1}}{\longrightarrow} Q_{k(1)} \stackrel{g_{0}}{\longrightarrow} P_{k(0)} \stackrel{f_{0}}{\longrightarrow} Q_{k(0)}$.

ПРЕДЛОЖЕНИЕ 2.7 (Лэчер [47]). Компакт X имеет шейn точки, тогда и только тогда, когда он является пределом обратной последовательности отображений между кусочно линейными шарами.

Компакты, удовлетворяющие одному из эквивалентных условий предложения 2.7, в литературе называются клеточноподобными. Отображения с клеточноподобными прообразами точек будут рассмотрены в пунктах (d) и (е) теоремы 3.15 . 
ДокАЗАТЕЛЬСтво. Предположим, что $X$ - предел обратной последовательности полиэдров $P_{i}$. Для доказательства импликации "тогда" достаточно заметить, что если $P_{i}$ стягиваем, $f_{i}: P_{i+1} \rightarrow P_{i}$ - связующее отображение и $h_{i}-$ нульгомотопия $\operatorname{id}_{P_{i}}$, то композиции $h_{i} f_{i}$ и $f_{i} h_{i+1}$ гомотопны.

Наоборот, предположим, что $X$ имеет шейп точки. Тогда по предложению 2.6 для каждого $i$ существует $j=j(i)>i$ такое, что связующее отображение $P_{j} \rightarrow P_{i}$ нульгомотопно. Тогда оно пропускается через включение $P_{j}$ в конус $C P_{j}$. Пусть $j_{0}=0$ и $j_{k+1}=j\left(j_{k}\right)$ и положим $Q_{k}=C P_{j_{k}}$. Тогда $X-$ предел обратной последовательности отображений $Q_{k+1} \rightarrow P_{j_{k}} \subset Q_{k}$. Каждый конус $Q_{k}$ стягиваем, поэтому регулярная окрестность его вложенного экземпляра в некотором евклидовом пространстве является кусочно линейным шаром $B^{n_{k}}$. Тогда $X$ - предел обратной последовательности отображений $B^{n_{k+1}} \rightarrow Q_{k+1} \rightarrow Q_{k} \subset B^{n_{k}}$. Предложение 2.7 доказано.

Заметим, что предложение 2.6 не утверждает, будто $f: X \rightsquigarrow Y$ является стинродовской гомотопической эквивалентностью, если и только если оно представляется изоморфизмом в про-гомотопиях. Известно, что это так в конечномерном случае (см. теорему 3.9); общий случай остается открытым (см. частичные результаты в [23] и [48]). Следующая версия предложения 2.6 “для отображений" - другой результат в этом направлении.

ПрЕДЛОЖЕНИЕ 2.8. Пусть $X и Y-$ компакть.

(а) (Дыдак-Сигал) Всякий стинродовский гомотопический класс $f: X \rightsquigarrow Y$ разложи́м в композицию $h[i]$ вложения $i: X \hookrightarrow Z$ и стинродовской сильной деформационной ретракции (значит, в частности, стинродовской гомотопической эквивалентности) $h: Z \rightsquigarrow Y$.

(b) Пусть $f: X \hookrightarrow Y$ - отображение включения. Следующие утверждения эквивалентны:

(i) $f$ - стинродовская гомотопическая эквивалентность;

(ii) $X$ - стинродовский сильный деформационный ретракт $Y$;

(iii) $(X, Y)$ - обратный предел компактных полиэдралъных пар $\left(Q_{i}, P_{i}\right)$ таких, что если через $f_{[0, \infty)}: P_{[0, \infty)} \rightarrow Q_{[0, \infty)}$ обозначить отображение включения, то существует сохраняющее уровни отображение $g_{[0, \infty)}$ : $Q_{[0, \infty)}^{s} \rightarrow P_{[0, \infty)}$, где $Q_{i}^{s}=Q_{i+1}$ такое, что каждая композиция $g_{i} f_{i+1}$ : $P_{i+1} \rightarrow P_{i}$ равна связующему отображению и каждая композичия $f_{i} g_{i}: Q_{i+1} \rightarrow Q_{i}$ гомотопна неподвижно на $P_{i+1}$ связующему отображению.

Стинродовский гомотопический класс $h: Z \rightsquigarrow Y$ называется стинродовской сильной деформационной ретракцией, если $Y \subset Z$ и пара $(Z, Y)$ - предел обратной последовательности компактных полиэдральных пар $\left(Q_{i}, P_{i}\right)$ такой, что $h$ представляется собственной сильной деформационной ретракцией $Q_{[0, \infty)} \rightarrow P_{[0, \infty)}$

Часть (а) была доказана в [23] с помощью иных построений, занимающих там c. 24-26. Импликация (i) $\Rightarrow$ (ii) связана с [25; (1.15)] (см. также [23; теоремы 4.5, $\left.6.3^{\prime}\right]$ ). Идея "алгебраического" доказательства импликации (iii) $\Rightarrow$ (i) берет начало в [23; теорема 6.2], а построение, использованное в конце "геометрического" доказательства, фигурирует в [22; доказательство предложения (3.7.4)]. 
Доказательство. (а). По лемме $2.5\left(\mathrm{~b}_{0}\right),\left(\mathrm{b}_{1}\right) f$ может быть представлено сохраняющим уровни отображением $f_{[0, \infty)}: P_{[0, \infty)} \rightarrow Q_{[0, \infty)}$, где $\lim _{\longleftarrow} P_{i}=X$ и $\lim _{\leftarrow} Q_{i}=Y$. Каждое связующее отображение $P_{i+1} \rightarrow P_{i}$ продолжается до отображения $M C\left(f_{i+1}\right) \rightarrow M C\left(f_{i}\right) \cup Q_{[i, i+1]} \rightarrow M C\left(f_{i}\right)$ между цилиндрами отображений, которое также сужается до связующего отображения $Q_{i+1} \rightarrow Q_{i}$. Обозначим $R_{i}=M C\left(f_{i}\right)$; ясно, что $R_{[0, \infty)}$ собственно сильно деформационно ретрагируется на $Q_{[0, \infty)}$. Ретракция $h_{[0, \infty)}: R_{[0, \infty)} \rightarrow Q_{[0, \infty)}$ представляет стинродовскую сильную деформационную ретракцию $h: Z \rightsquigarrow Y$, где $Z=\lim R_{i}$. Очевидно, $f_{[0, \infty)}$ собственно гомотопно композиции отображения включения $P_{[0, \infty)} \hookrightarrow R_{[0, \infty)}$ c $h_{[0, \infty)}$.

(b). $\quad(\mathrm{i}) \Rightarrow(\mathrm{ii}) \Rightarrow\left(\right.$ iii). Представим $f$ собственным отображением $f_{[0, \infty)}$ : $P_{[0, \infty)} \rightarrow Q_{[0, \infty)}$. В предположении справедливости $(\mathrm{i}) f_{[0, \infty)}$ является собственной гомотопической эквивалентностью. Тогда, по доказательствам стандартных результатов гомотопической теории продолжения [14; следствие 1.4.10, теорема 1.4.11, упражнение 1.D.2], существует собственная сильная деформационная ретракция $Q_{[0, \infty)}$ на $P_{[0, \infty)}$. Это доказывает (ii). Импликация (ii) $\Rightarrow$ (iii) получается с использованием леммы $2.5(\mathrm{~b})$.

(iii) $\Rightarrow$ (i). Алгебраическое доказательство. Введем обозначение $(Q, P)$ для обратной последовательности $\cdots \rightarrow\left(Q_{1}, P_{1}\right) \rightarrow\left(Q_{0}, P_{0}\right)$; из условия вытекает, что $(Q, P)$ изоморфна $(P, P)$ в про-гомотопиях. Тогда по доказательству предложения $2.6(Y, X)$ имеет шейп $(X, X)$. Если $\varphi:(Y, X) \rightsquigarrow(X, X)$ и $\psi:(X, X) \rightsquigarrow(Y, X)$ таковы, что $\varphi \psi=\left[\operatorname{id}_{(X, X)}\right]$ и $\psi \varphi=\left[\operatorname{id}_{(Y, X)}\right]$, то $\psi: X \rightsquigarrow Y$, $\varphi: X \rightsquigarrow X$ и включение $i: Y \rightarrow X$ удовлетворяют $\psi \varphi[i]=\left[\operatorname{id}_{Y}\right]$ и $[i] \psi \varphi=\left[\operatorname{id}_{X}\right]$. Таким образом, $[i]$ - стинродовская гомотопическая эквивалентность.

Геометрическое доказательство. Поскольку $\left.g_{i}\right|_{P_{i+1}}$ равняется связующему отображению, цилиндр отображения $M C\left(g_{i}\right)$ содержит $P_{[i, i+1]}$. Гомотопия между $f_{i} g_{i}$ и связующим отображением доставляет гомотопическую эквивалентность $\varphi: M C\left(g_{i}\right) \rightarrow Q_{[i, i+1]}$, сужающуюся в тождественное отображение на $Q_{i} \cup P_{[i, i+1]}$. Из $Q_{i}^{\prime}:=M C\left(g_{i}\right)$ и $P_{i}^{\prime}:=P_{[i, i+1]}$ можно составить обратные последовательности со связующими отображениями $Q_{i+1}^{\prime} \stackrel{\pi \varphi}{\longrightarrow} Q_{i+1} \subset Q_{i}^{\prime}$ и $P_{i+1}^{\prime} \stackrel{\pi}{\rightarrow} P_{i+1} \subset P_{i}^{\prime}$, где каждое $\pi$ проектирует цилиндр отображения на его пространство значений. Так как $\cdots \rightarrow\left(Q_{1}^{\prime}, P_{1}^{\prime}\right) \rightarrow\left(Q_{0}^{\prime}, P_{0}^{\prime}\right)$ эквивалентно $\cdots \rightarrow\left(Q_{1}, P_{1}\right) \rightarrow\left(Q_{0}, P_{0}\right)$ относительно отношения эквивалентности из предложения 2.6 (iii), то отображение включения $f_{[0, \infty)}^{\prime}: P_{[0, \infty)}^{\prime} \rightarrow Q_{[0, \infty)}^{\prime}$ представляет стинродовский гомотопический класс отображения $f$. С другой стороны, каждое $Q_{i}^{\prime}$ очевидно сдавливается на $P_{i}^{\prime}$.

Сильная деформационная ретракция $Q_{i}^{\prime}$ на $P_{i}^{\prime}$ доставляет деформационную ретракцию $Q_{i}^{\prime} \times I$ на $P_{i}^{\prime} \times I \cup Q_{i}^{\prime} \times \partial I$. Аналогично, $Q_{[i, i+1]}^{\prime}$ деформационно ретрагируется на $P_{[i, i+1]}^{\prime} \cup Q_{i+1}^{\prime} \cup Q_{i}^{\prime}$. Компонируя последние ретракции с ретракциями $Q_{i}^{\prime} \rightarrow P_{i}^{\prime}$, получаем собственную деформационную ретракцию $r: Q_{[0, \infty)}^{\prime} \rightarrow P_{[0, \infty)}^{\prime} \cup Q_{\mathbb{N}}^{\prime} \rightarrow P_{[0, \infty)}^{\prime}$. Таким образом, $r-$ собственная гомотопическая эквивалентность, обращающая собственный гомотопический класс включения $f_{[0, \infty)}^{\prime}$. Предложение 2.8 доказано.

Отображение $f: X \rightarrow Y$ (соответственно стинродовский гомотопический класс $[F]: X \rightsquigarrow Y$ ) между компактами с базисными точками $x \in X, y \in Y$ на- 
зывается пунктированным, если оно сохраняет базисную точку, т. е. $f(x)=y$ (соответственно если собственное отображение $F: P_{[0, \infty)} \rightarrow Q_{[0, \infty)}$ сохраняет базисный луч, т. е. $F\left(p_{[0, \infty)}\right) \subset q_{[0, \infty)}$, где $p_{i}$ и $q_{i}-$ образы $x$ и $y$ в $P_{i}$ и $\left.Q_{i}\right)$. Множество $[(X, x),(Y, y)]$ пунктированных стинродовских гомотопических классов наделяется естественной структурой пунктированного множества (т. е. множества с отмеченной точкой).

ПРЕДЛОЖЕНИЕ 2.9. Пусть $X$ и $Y$ - связные компакты с базисными точками $x$ и $y$, и пусть $f:(X, x) \rightsquigarrow(Y, y)$ - стинродовский гомотопический класс. Если непунктированный класс $\bar{f}: X \rightsquigarrow Y$ отображения $f$ является стинродовской гомотопической эквивалентностъю, то и $f$ тоже стинродовская гомотопическая эквивалентность.

Это доказано в $[23 ; 4.6]$ и $[25 ; 1.16$ (см. также $1.14,1.15)]$.

ДокАЗАтЕльСтво. В силу пунктированной версии предложения 2.8 (а), мы можем предположить, не теряя общности, что $f$ - отображение включения (тогда, в частности, $x=y$ ). Тогда, в силу импликации (i) $\Rightarrow$ (ii) из предложения 2.8 (b), $X$ - стинродовский сильный деформационный ретракт компакта $Y$. Но из этого вытекает, что $(X, x)$ - стинродовский сильный деформационный ретракт пунктированного компакта $(Y, y)$. Предложение 2.9 доказано.

Компакт $X$ связен по Стинродy, если существует только один стинродовский гомотопический класс из точки в $X$. Предложение 2.9 показывает, что для компакта, связного по Стинроду, нет разницы между пунктированным и непунктированным шейпом.

Пример 2.10 (синусоида топологов). Пусть $X \subset[0,1] \times[-1,1]$ - замыкание графика $\sin (1 / x)$, где $x$ пробегает $(0,1]$. Тогда $X$ стинродовски связен (в действительности, даже клеточноподобен), но не линейно связен.

Связные по Стинроду компакты будут рассмотрены подробнее в 8 . Так, из теоремы 8.7 (принадлежащей Красинкевичу и Минцу) следует, что компакт $X$ связен по Стинроду, если и только если всякие два отображения $\mathrm{pt} \rightarrow X$ представляют один и тот же стинродовский гомотопический класс. В частности, линейно связные компакты связны по Стинроду.

С другой стороны, как подмечено в [49; замечание 10.1], множество стинродовских гомотопических классов pt $\rightsquigarrow X$, представимых отображениями, не является шейповым инвариантом: если $\alpha:$ pt $\rightsquigarrow X$ не представи́м отображением, то предста́вим его сохраняющим уровни отображением $f:[0, \infty) \rightarrow P_{[0, \infty)}$, где $X=\lim _{\longleftarrow} P_{i}$. Тогда $Y:=X \cup f([0, \infty))$ стинродовски гомотопически эквивалентно $X$, но $\alpha$ : pt $\rightsquigarrow Y$ теперь уже представлен посредством $\left.f\right|_{\{0\}}$.

\section{§ 3. Гомотопические группы}

Определение множества $[X, Y]$ стинродовских гомотопических классов $X \rightsquigarrow Y$ обобщается на пары компактов очевидным образом, если опираться на то, что всякая пара компактов есть обратный предел пар компактных полиэдров. Определим тогда стинродовское гомотопическое множество: $\pi_{n}(X ; x)=\left[\left(S^{n}, \mathrm{pt}\right),(X, x)\right]$. Таким образом (см. предложение $\left.2.4(\mathrm{a})\right), \pi_{n}(X ; x)$ 
может быть отождествлено с множеством классов сохраняющих уровни отображений

$$
\left(S^{n} \times[0, \infty), \text { pt } \times[0, \infty)\right) \rightarrow\left(P_{[0, \infty)}, p_{[0, \infty)}\right)
$$

относительно сохраняющей уровни гомотопии, где $P=\left(\cdots \stackrel{p_{1}}{\longrightarrow} P_{1} \stackrel{p_{0}}{\longrightarrow} P_{0}\right)-$ обратная последовательность компактных полиэдров и кусочно линейных отображений с пределом $X$, а $p_{[0, \infty)}$ - телескоп образов $p_{i}$ базисной точки $x$ в полиэдрах $P_{i}$. Согласно предложению 2.3 (а), если $X$ - полиэдр, то отображение $\stackrel{\Delta}{\tau}: \stackrel{\Delta}{\pi}_{n}(X ; x) \rightarrow \pi_{n}(X ; x)$ является биекцией.

Групповые операции (для $n \geqslant 1$ ) и индуцированные отображения $f_{*}$, где $f-$ пунктированное отображение или пунктированный стинродовский гомотопический класс, определяются очевидным образом. Мы опускаем базисную точку в обозначениях и пишем $\pi_{n}(X)$, когда это не приводит к путанице. Далее, пусть $\pi_{n}(X, A ; x)=\left[\left(D^{n}, \partial D^{n}, \mathrm{pt}\right),(X, A, x)\right]$ для любой пары компактов $(X, A)$ с базисной точкой $x \in A$ и любого $n>0$. Более общо, будем допускать произвольный стинродовский гомотопический класс $x: \mathrm{pt} \rightsquigarrow A$ в качестве базисной точки. Мы также пишем $\pi_{n}(X, A)$, когда базисная точка явно не используется. Групповые операции в $\pi_{n}(X, A)$ определяются очевидным способом для $n \geqslant 2$. Имеется действие $\pi_{1}(A)$ на $\pi_{n}(X)$ при $n \geqslant 1$ и на $\pi_{n}(X, A)$ при $n \geqslant 2$ и действие $\pi_{1}(X)$ на $\pi_{1}(X, A)$. Мы оставляем $\pi_{0}(X, A)$ неопределенным.

Топология на $\pi_{n}(X)$. База топологии на $\pi_{n}(X)$ задается прообразами точек отображений $f_{*}: \pi_{n}(X) \rightarrow \pi_{n}(P)$, индуцированных всеми отображениями $f: X \rightarrow P$, где $P$ - компактный полиэдр. Очевидно, групповые операции (при $n>0$ ), так же как и все индуцированные отображения, непрерывны в этой топологии. Если $X$ - предел обратной последовательности полиэдров $P_{i}$, то по лемме 2.1 (а) всякое отображение $f$ из $X$ в полиэдр $Q$ продолжается до отображения $P_{[k, \infty]} \rightarrow Q$ для некоторого $k$ и, таким образом, $f_{*}$ пропускается через $\left(p_{k}^{\infty}\right)_{*}$. Следовательно, в качестве базы топологии на $\pi_{n}(X)$ можно также взять прообразы точек всех отображений $\left(p_{i}^{\infty}\right)_{*}$, индуцированных проекциями $p_{i}^{\infty}: X \rightarrow P_{i}$.

Очевидно, что топологическая группа (пунктированное пространство при $n=0) \pi_{n}(X, x)$ является шейповым инвариантом пары $(X, x)$. Когда $X$ связен по Стинроду (т. е. $\pi_{0}(X)=\mathrm{pt}$ ), по предложению 2.9 она также является шейповым инвариантом $X$. Кроме того, как непунктированное пространство $\pi_{0}(X)$ является, очевидно, шейповым инвариантом $X$.

Производный предел. Пусть $G=\left(\cdots \stackrel{p_{1}}{\longrightarrow} G_{1} \stackrel{p_{0}}{\longrightarrow} G_{0}\right)$ - обратная последовательность групп. Группа $\prod G_{i}$ действует на себе по формуле

$$
\left(x_{0}, x_{1}, \ldots\right)\left[\left(g_{0}, g_{1}, \ldots\right)\right]=\left(x_{0} g_{0} p_{0}\left(x_{1}^{-1}\right), x_{1} g_{1} p_{1}\left(x_{2}^{-1}\right), \ldots\right) .
$$

Стабилизатор единицы при этом действии может быть отождествлен с обратным пределом $\lim G_{i}$. Мы определяем $\lim ^{1} G_{i}$ как пространство орбит этого действия, рассмотренное как пунктированное множество. Заметим, что отображение $f: \prod G_{i} \rightarrow \prod G_{i}$ пунктированных множеств, определенное формулой $f\left(g_{0}, g_{1}, \ldots\right)=\left(g_{0}^{-1} p_{0}\left(g_{1}\right), g_{1}^{-1} p_{1}\left(g_{2}\right), \ldots\right)$, эквивариантно относительно правого регулярного действия $\prod G_{i}$ на области определения и определенного выше действия на пространстве значений. Имеем $\lim G_{i}=\operatorname{ker} f$, тогда как $\lim ^{1} G_{i}$ 
тривиален, если и только если $f$ сюръективно. Когда $G_{i}$ абелевы, $f$ является гомоморфизмом, a $\lim ^{1} G_{i}$ может быть отождествлен с coker $f$ и тем самым наделяется структурой абелевой группы. ${ }^{14}$

Снабдим $\lim G_{i}$ топологией обратного предела, которая индуцируется топологией произведения на произведении $\prod G_{i}$ дискретных групп, a $\lim ^{1} G_{i}-$ фактортопологией, которая всегда антидискретна, так как замена конечного числа компонент любого $\left(g_{1}, g_{2}, \ldots\right) \in \prod G_{i}$ не изменяет его орбиты.

Если группы $G_{i}$ абелевы, то $G \mapsto \lim ^{1} G_{i}$ есть производный функтор функтора обратного предела $G \mapsto \underset{\lim }{\longleftarrow} G_{i}$ в смысле гомологической алгебры. В самом деле, можно показать, что $\lim ^{1} G_{i}$ тривиален, если все связующие отображения в $G$ - эпиморфизмы, а значит,

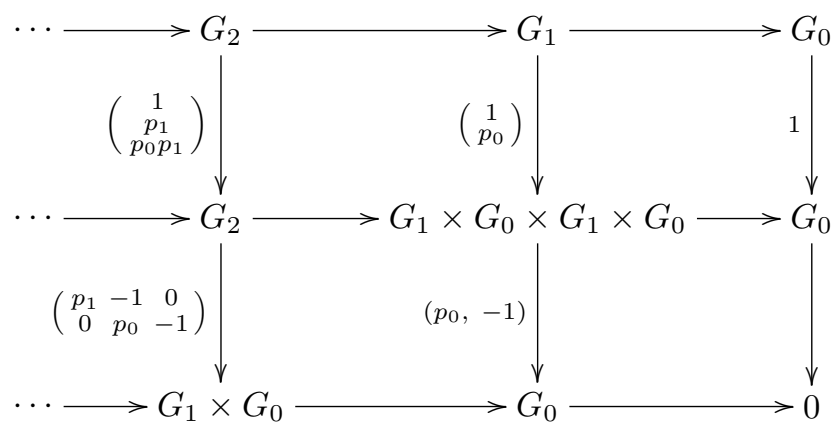

является lim-ацикличной резольвентой $G$ (ее связывают с именем Я.-Э. Роса). В абелевом случае теорема $3.1(\mathrm{~d})$ ниже - это обычная длинная точная последовательность для производных функторов, а теорема 4.1 (iii) ниже (гомологическая версия теоремы 3.1 (b)) - это формула универсальных коэффициентов для производных функторов.

Tеорема 3.1. (а) [50] Если $(X, A)$ - пара компактов с базисной точкой $x \in A$ (или, более общо, $x: \mathrm{pt} \rightsquigarrow A)$, то имеется точная последовательность пунктированных множеств

$$
\cdots \rightarrow \pi_{2}(X, A) \rightarrow \pi_{1}(A) \rightarrow \pi_{1}(X) \rightarrow \pi_{1}(X, A) \rightarrow \pi_{0}(A) \rightarrow \pi_{0}(X),
$$

отображения которой слева от $\pi_{1}(X)$ (соответственно $\left.\pi_{2}(X)\right)$ являются гомоморфизмами групп (соответственно правых $\mathbb{Z} \pi_{1}(A)$-модулей). Кроме того, $\pi_{2}(X) \rightarrow \pi_{2}(X, A)$ также $\pi_{1}(A)$-эквивариантен, $\partial: \pi_{2}(X, A) \rightarrow \pi_{1}(A)$ является скрещенным модулем ${ }^{15}, \pi_{1}(X) \rightarrow \pi_{1}(X, A)$ является $\pi_{1}(X)$-эквивариантным относительно правого регулярного действия на $\pi_{1}(X)$ и нетривиальные прообразы точек $\pi_{1}(X, A) \rightarrow \pi_{0}(A)$ совпадают с орбитами действия $\pi_{1}(X)$.

(b) [33], [50], [51], [22; теорема (5.2.1)], [52] Eсли $(X, A)$ - предел обратной последовательности пар $\left(P_{i}, Q_{i}\right)$ связных компактных полиэдров, то имеется

\footnotetext{
${ }^{14}$ Если для каждого $i$ и всякого $x_{i} \in G_{i}$ найдется $j>i$ такое, что образ всякого $y_{j} \in G_{j}$ в $G_{i}$ коммутирует с $x_{i}$, то на $\lim ^{1} G_{i}$ имеется структура полугруппы; и если дополнительно каждая $G_{i}$ разрешима, она дополняется до групповой структуры [48].

15 т. е. $\partial(s \cdot g)=g^{-1}(\partial s) g$ для $g \in \pi_{1}(A), s \in \pi_{2}(X, A)$ и $s^{-1} t s=t \cdot(\partial s)$ для $s, t \in \pi_{2}(X, A)$.
} 
точная последовательность

$$
1 \rightarrow \lim ^{1} \pi_{n+1}\left(P_{i}, Q_{i}\right) \rightarrow \pi_{n}(X, A) \rightarrow \lim _{\longleftarrow} \pi_{n}\left(P_{i}, Q_{i}\right) \rightarrow 1
$$

пунктированных множеств при $n \geqslant 1$ или $A=Q_{i}=\mathrm{pt} u n=0$, отображения которой суть групповые гомоморбизмы при $n \geqslant 2$ или $A=Q_{i}=$ pt $u n=1$.

(c) Заключение части (b) справедливо также, когда $\left(P_{i}, Q_{i}\right)$ - пары связных компактов.

(d) [53; предложение 2.3 в гл. IX (см. также гл. XI)], [27; с. 168] Ecли $1 \rightarrow$ $K_{i} \stackrel{j_{i}}{\longrightarrow} G_{i} \stackrel{f_{i}}{\longrightarrow} Q_{i} \rightarrow 1$ - точная последовательность обратных последовательностей групп (m.е. $j_{i}$ и $f_{i}$ коммутируют со связующими отображениями), то имеется точная последовательность пунктированных множеств

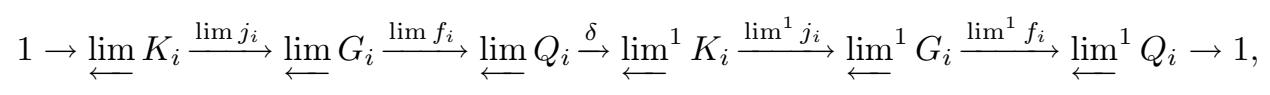

где отображение $\delta$ является $\lim Q_{i}$-эквивариантным относительно правого

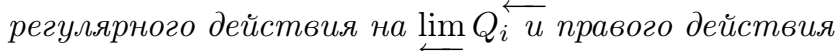

$$
\left[\left(k_{0}, k_{1}, \ldots\right)\right] \cdot\left(g_{0} K_{0}, g_{1} K_{1}, \ldots\right)=\left[\left(g_{0}^{-1} k_{0} p_{0}\left(g_{1}\right), g_{1}^{-1} k_{1} p_{1}\left(g_{2}\right), \ldots\right)\right]
$$

на $\lim ^{1} K_{i}$, причем нетривиальные прообразы точек при $\lim ^{1} j_{i}$ совпадают с орбитами действия

$\left(\mathrm{d}^{\prime}\right)$ [54] Если $1 \rightarrow H_{i} \stackrel{j_{i}}{\longrightarrow} G_{i} \stackrel{f_{i}}{\longrightarrow} G_{i} / H_{i} \rightarrow 1$ - точная последовательность обратных последовательностей пунктированных множеств, причем $G_{i}$ и $H_{i}$ являются группами, а $j_{i}-$ их гомоморфизмами, то имеется точная последовательность пунктированных множеств

$$
1 \rightarrow \lim _{\longleftarrow} H_{i} \stackrel{\lim j_{i}}{\longleftrightarrow} \lim _{\longleftarrow} G_{i} \stackrel{\lim f_{i}}{\longrightarrow} \underset{\lim }{\longleftarrow}\left(G_{i} / H_{i}\right) \stackrel{\delta}{\longrightarrow} \lim ^{1} H_{i} \stackrel{\lim ^{1} j_{i}}{\longleftrightarrow} \lim ^{1} G_{i},
$$

где нетривиальные прообразы точек при $\delta$ совпадают с орбитами очевидного действия $\lim _{\longleftarrow} G_{i}$ на $\underset{\lim }{\longleftarrow}\left(G_{i} / H_{i}\right)$.

Доказательство показывает, что все гомоморфизмы в теореме 3.1 непрерывны и естественны.

ДокАзАтЕЛьство. (а) Это вытекает из гомотопической точной последовательности пары для собственных отображений, которая проверяется непосредственно.

(b) Непосредственная проверка показывает, что последовательность сохраняющих уровни пунктированных гомотопических классов

$$
\begin{aligned}
\cdots \rightarrow\left[\left(D^{n+1}, S^{n}\right) \times \mathbb{N},\left(P_{\mathbb{N}}, Q_{\mathbb{N}}\right)\right]_{\ell}^{*} & \stackrel{b}{\rightarrow}\left[\left(D^{n}, S^{n-1}\right) \times[0, \infty),\left(P_{[0, \infty)}, Q_{[0, \infty)}\right)\right]_{\ell}^{*} \\
& \stackrel{c}{\rightarrow}\left[\left(D^{n}, S^{n-1}\right) \times \mathbb{N},\left(P_{\mathbb{N}}, Q_{\mathbb{N}}\right)\right]_{\ell}^{*} \stackrel{a}{\rightarrow} \cdots
\end{aligned}
$$

точна слева от стрелки $a$ с $n=1$ или с $Q_{i}=$ pt и $n=0$. "Пунктированность" означает, что $\mathrm{pt} \times[0, \infty)$ всегда отображается в $p_{[0, \infty)}$, a $\mathrm{pt} \times \mathbb{N}$ всегда отображается в $p_{\mathbb{N}}$. Здесь $c$ определено ограничением, а $b$ - используя очевидное отождествление между $\left[\left(D^{n+1}, S^{n}\right) \times \mathbb{N},\left(P_{\mathbb{N}}, Q_{\mathbb{N}}\right)\right]_{\ell}^{*}$ и

$$
\left[\left(D^{n} \times[0, \infty), S^{n-1} \times[0, \infty) \cup D^{n} \times \mathbb{N}\right),\left(P_{[0, \infty)}, Q_{[0, \infty)}\right)\right]_{\ell}^{*}
$$

для $n>0$ или при $n=0$ и $Q_{i}=\mathrm{pt}$. 
Наконец, а может быть отождествлено с отображением $f: \prod \pi_{n}\left(P_{i}, Q_{i}\right) \rightarrow$ $\prod \pi_{n}\left(P_{i}, Q_{i}\right)$, заданным как $\left(g_{1}, g_{2}, \ldots\right) \mapsto\left(g_{1}-p_{1 *}\left(g_{2}\right), g_{2}-p_{2 *}\left(g_{3}\right), \ldots\right)$ в абелевом случае (при $n \geqslant 3$ или $Q_{i}=$ pt и $\left.n=2\right)$ и как $\left(g_{1}, g_{2}, \ldots\right) \mapsto\left(g_{1} p_{1 *}\left(g_{2}^{-1}\right)\right.$, $\left.g_{2} p_{2 *}\left(g_{3}^{-1}\right), \ldots\right)$ в групповом случае (при $n \geqslant 2$ или $Q_{i}=$ pt и $\left.n=1\right)$. В случае пунктированных множеств $a$ может быть определено аналогично, если его пространство значений $\left[\left(D^{1}, S^{0}\right) \times \mathbb{N},\left(P_{\mathbb{N}}, Q_{\mathbb{N}}\right)\right]_{\ell}^{*}$ при $n=1$ (соответственно $\left[S^{0} \times \mathbb{N}, P_{\mathbb{N}}\right]_{\ell}^{*}$ при $Q_{i}=$ pt и $\left.n=0\right)$ подправить перемещением базисной точки в $D^{1} \backslash S^{0}$, вопреки стандартному соглашению (соответственно заменить непунктированным гомотопическим множеством $\left.\left[S^{0} \times \mathbb{N}, P_{\mathbb{N}}\right]_{\ell}\right)$.

Отображения в рассматриваемой длинной точной последовательности слева от $a: \prod \pi_{n}\left(P_{i}, Q_{i}\right) \rightarrow \prod \pi_{n}\left(P_{i}, Q_{i}\right)$ (не включительно!) суть групповые гомоморфизмы, если $n=2$ или $Q_{i}=$ pt и $n=1$. Теперь искомое утверждение следует из того, что $\lim _{n} \pi_{n}\left(P_{i}, Q_{i}\right)=\operatorname{ker} f$, а в абелевом случае также $\lim ^{1} \pi_{n+1}\left(P_{i}, Q_{i}\right)=\operatorname{coker} f$. В неабелевом случае, записывая крайний пра-

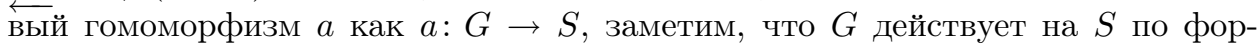
муле из определения $\lim ^{1}$ и $a$ эквивариантен относительно этого действия на $S$ и правого регулярного действия на $G$. Орбиты этого действия суть в точности нетривиальные прообразы точек последующего (вправо) отображения $b$.

(c) Мы опускаем базисные точки. Для ясности обратная последовательность компактов $\cdots \stackrel{p_{1}}{\longrightarrow} P_{1} \stackrel{p_{0}}{\longrightarrow} P_{0}$ будет обозначаться через $\cdots \stackrel{q_{1}}{\longrightarrow} C_{1} \stackrel{q_{0}}{\longrightarrow} C_{0}$.

Представим каждый $C_{i}$ как предел обратной последовательности $P^{(i)}=$ $\left(\cdots \stackrel{p_{1}^{(i)}}{\longrightarrow} P_{1}^{(i)} \stackrel{p_{0}^{(i)}}{\longrightarrow} P_{0}^{(i)}\right)$, где $P_{j}^{(i)}-$ компактные полиэдры, $p_{j}^{(i)}-$ кусочно линейные отображения. Отображения $q^{i}$ дают стинродовские гомотопические классы, представленные собственными отображениями $q_{[0, \infty)}^{i}: P_{[0, \infty)}^{(i+1)} \rightarrow P_{[0, \infty)}^{(i)}$. По лемме $2.5\left(\mathrm{~b}_{0}\right)$, после перехода к подпоследовательности $P^{(1)}$ мы можем предполагать, что $q_{[0, \infty)}^{0}$ сохраняет уровни. Продолжая эту процедуру, мы можем предполагать, что все $q_{[0, \infty)}^{i}$ сохраняют уровни. Рассмотрим $T_{J}:=$ $\left(\cdots \stackrel{q_{J}^{1}}{\longrightarrow} P_{J}^{(1)} \stackrel{q_{J}^{0}}{\longrightarrow} P_{J}^{(i)}\right)$ и введем обозначение $T_{J \times K}$ для $\left(T_{J}\right)_{K}$. По доказательству (b) искомая точная последовательность имеет место при условии, что $\pi_{n}(X)$ заменены группой $G$ послойных гомотопических классов отображений $F: S^{n} \times[0, \infty) \times[0, \infty) \rightarrow T_{[0, \infty) \times[0, \infty)}$, расслоенных над $[0, \infty) \times[0, \infty)$.

Рассмотрим диагональный телескоп-“лестницу"

$$
T^{\Delta}=T_{[0,1] \times 0} \cup T_{1 \times[0,1]} \cup T_{[1,2] \times 1} \cup T_{2 \times[1,2]} \cup \cdots=Q_{[0, \infty)},
$$

где $Q_{2 i}=P_{i}^{(i)}$ и $Q_{2 i+1}=P_{i+1}^{(i)}$. Очевидно, что $X$ гомеоморфен $\underset{\lim }{\longleftarrow} Q_{i}$. С другой стороны, ограничение послойного отображения $F$ на $T^{\Delta}$ доставляет сохраняющее уровни $F^{\Delta}: S^{n} \times[0, \infty) \rightarrow Q_{[0, \infty)}$, и наоборот, каждое $F^{\Delta}$ продолжается до такого $F$, с использованием гомотопий, аналогичных $\Pi_{t}$. Отсюда следует, что $G \simeq \pi_{n}(X)$.

(d) Для разнообразия приведем геометрическое доказательство в случае, когда каждая $G_{i}$ допускает классифицирующее пространство с компактным 3 -мерным остовом $B G_{i}$, а каждая $Q_{i}$ - с компактным 4-мерным остовом $B Q_{i}$; общий случай счетных групп получается аналогичным образом, если использовать определения, данные в конце $§ 7$. Рассматривая цилиндр отображения, 
мы можем предполагать, что $B G_{i} \subset B Q_{i}$, причем это включение индуцирует заданную сюръекцию $G_{i} \rightarrow Q_{i}$ на $\pi_{1}$. Имеем $\pi_{2}\left(B G_{i}\right)=0=\pi_{2}\left(B Q_{i}\right)$ и $\pi_{3}\left(B Q_{i}\right)=0$, откуда $\pi_{3}\left(B Q_{i}, B G_{i}\right)=0$. Пусть $\cdots \rightarrow\left(B G_{1}, B Q_{1}\right) \rightarrow\left(B G_{0}, B Q_{0}\right)-$ обратная последовательность, связующие отображения которой индуцируют заданные $G_{i+1} \rightarrow G_{i}$ (и, следовательно, также $Q_{i+1} \rightarrow Q_{i}$ ). Пусть $(X, A)=$ $\lim \left(B Q_{i}, B G_{i}\right)$. Тогда часть (b) отождествляет члены точной последовательности из (а) с членами последовательности из формулировки (d). Сюръективность крайнего правого отображения вытекает из того, что, в обозначениях пункта $(\mathrm{b})$, если $\pi_{0}\left(P_{i}\right), \pi_{0}\left(Q_{i}\right)$ и $\pi_{1}\left(P_{i}, Q_{i}\right)$ тривиальны, то $\pi_{0}(A) \rightarrow \pi_{0}(X)$ сюръективно по доказательству (b).

(d') Аналогично (d); см. также доказательство леммы 3.7 (a) ниже.

ПримеР 3.2. В качестве иллюстрации к теореме 3.1 (d) вычислим группу $\lim ^{1}(\cdots \stackrel{p}{\rightarrow} \mathbb{Z} \stackrel{p}{\rightarrow} \mathbb{Z})$. Данная обратная последовательность включается в короткую точную с $G=(\cdots \stackrel{\text { id }}{\longrightarrow} \mathbb{Z} \stackrel{\mathrm{id}}{\longrightarrow} \mathbb{Z})$ и $Q=\left(\cdots \stackrel{\bmod p^{2}}{\longrightarrow} \mathbb{Z} / p^{2} \stackrel{\bmod p}{\longrightarrow} \mathbb{Z} / p \rightarrow 1\right)$. Очевидно, $\lim ^{1} G$ тривиален, поэтому $\lim ^{1}(\cdots \stackrel{p}{\longrightarrow} \mathbb{Z} \stackrel{p}{\rightarrow} \mathbb{Z}) \simeq \underset{\lim }{\longleftarrow} / \stackrel{\lim }{\longleftarrow}=\mathbb{Z}_{p} / \mathbb{Z}$.

Сходство между этим вычислением и доказательством леммы 3.3 ниже будет проанализировано и применено в доказательстве теоремы 8.4.

Говорят, что обратная последовательность $\cdots \rightarrow G_{1} \rightarrow G_{0}$ удовлетворяет условию Миттаг-Леффлера, если для каждого $i$ найдется такое $j>i$, что для каждого $k>j$ образ $G_{k} \rightarrow G_{i}$ равен образу $G_{j} \rightarrow G_{i}$. Если $G_{i}$ - группы, то в качестве несложного, но поучительного упражнения читатель может проверить, что выполнение условия Миттаг-Леффлера влечет тривиальность $\lim ^{1} G_{i}$. Следующая лемма показывает, что обратное справедливо, если каждая $G_{i}$ счетна.

Лемма 3.3 [55], [56], [29], [57]. Пусть $G_{i}$ - обратная последовательность счетных групп. Если $\lim ^{1} G_{i}$ счетен (например, тривиален), то $G_{i}$ удовлетворяют условию Миттаг-Лебблера.

Мы приводим доказательство, поскольку оно понадобится далее в нескольких местах. Приводимое изложение наиболее близко к [55], где лемма 3.3 сформулирована при дополнительном предположении.

ДоказАтельство. Допустим, что $G_{i}$ не удовлетворяют условию МиттагЛеффлера. Тогда существует $k$ такое, что образы $A_{i}$ групп $G_{k+i}$ в $G_{k}$ не стабилизируются. Так как каждое отображение $G_{i} \rightarrow A_{i}$ сюръективно, то $\lim ^{1} G_{i} \rightarrow$ $\lim ^{1} A_{i}$ сюръективно, поэтому $\lim ^{1} A_{i}$ счетен. Если $\left(g_{0}, g_{1}, \ldots\right)$ и $\left(h_{0}, \overleftarrow{h_{1}}, \ldots\right)-$ элементы $\prod A_{i}$, представляющие один и тот же элемент $\lim ^{1} A_{i}$, то каждый $g_{i}$ имеет вид $x_{i} h_{i} x_{i+1}^{-1}$ для некоторых $\left(x_{0}, x_{1}, \ldots\right) \in \prod A_{i}$. Тогда каждое произведение $g_{0} \cdots g_{n}$ равно $x_{0} h_{0} \cdots h_{n} x_{n+1}^{-1}$. Следовательно, $\left(g_{0} A_{1}, g_{0} g_{1} A_{2}, \ldots\right)$ и $\left(h_{0} A_{1}, h_{0} h_{1} A_{2}, \ldots\right)$ лежат в одной и той же орбите левого действия $A_{0}$ на $A:=\lim A_{0} / A_{i}$. Поскольку это рассуждение допускает обращение, мы получаем биекцию между $\lim ^{1} A_{i}$ и множеством орбит $A_{0} \backslash A$. Однако $A$ допускает биекцию с несчетным множеством $\left(A_{0} / A_{1}\right) \times\left(A_{1} / A_{2}\right) \times \cdots$, поэтому $A_{0} \backslash A$ должно быть несчетно. Лемма 3.3 доказана. 
Говорят, что обратная последовательность пунктированных множеств $G_{i}$ удовлетворяет двойственному условию Миттаг-Лефблера, если существует такое $k$, что для каждого $j>k$ существует $i>j$, для которого ядро отображения из $G_{i}$ в $G_{k}$ равно ядру отображения из $G_{i}$ в $G_{j}$ (cp. [58]). Когда это условие выполнено, легко видеть, что $\lim G_{i}$ отображается инъективно в некоторое $G_{k}$.

Лемма 3.4. Пусть $G_{i}$ - обратная последовательность пунктированных множеств. B пунктах (b) u (c) дополнительно предположим, что или $G_{i}$ является обратной последовательностъю групп, или же каждое $G_{i}$ конечно.

(а) $Е с л и G_{i}$ удовлетворяет условию Миттаг-Лефблера и $\lim G_{i}=0$, то для каждого $i$ найдется такое $j>i$, что $G_{j} \rightarrow G_{i}$ тривиально.

(b) Ecли $\lim G_{i}$ дискретен, он отображается ингективно в некоторое $G_{k}$.

(c) Пусть $\overleftarrow{G}_{i}$ удовлетворяют условию Миттаг-Лефблера. Если $\lim G_{i} \partial и с-$ кретен, то $G_{i}$ удовлетворяют двойственному условию Миттаг-Лефблера.

Обратные утверждения к (a), (b) и (c) верны, причем очевидны. Дополнительное предположение в пунктах (b) и (c) может быть опущено, если $\lim G_{i}$, рассмотренный как обратный предел дискретных равномерных пространств, предположить дискретным как равномерное пространство.

Часть (b) пригодится немного позже (в теореме 3.10 и также в §6). Часть (c) будет использована в теореме 3.12 .

ДоказАтельство. (а) Пусть $L_{i}$ обозначает стабильный образ множеств $G_{j}$, $j>i$, в $G_{i}$. Тогда $L_{i+1}$ отображается на $L_{i}$ и, следовательно, $\lim L_{j}$ отображается на $L_{i}$. Композиция $\lim L_{j} \rightarrow L_{i} \subset G_{i}$ пропускается через $\lim G_{i}$, поэтому $L_{i}$ тривиален.

(b) Если $g_{1}, g_{2}, \ldots$ - последовательность элементов предела $\underset{\lim }{\longleftarrow} G_{i}$ такая, что каждое $g_{i}$ отображается тривиально в $G_{i}$, то эта последовательность сходится к тождественному отображению в топологии обратного предела. Остается рассмотреть случай конечных множеств. Предположим, что $g_{1}, h_{1}, g_{2}, h_{2}, \ldots$ последовательность элементов предела $\lim G_{i}$ такая, что $g_{i}$ и $h_{i}$ отображаются в один и тот же элемент $G_{i}$. Так как $\longleftarrow \lim _{\longleftarrow} G_{i}$ - компакт, то существует бесконечная возрастающая последовательность $n_{i}$ такая, что $g_{n_{i}}$ и $h_{n_{i}}$ сходятся к некоторым $g$ и $h$ соответственно. Тогда $g=h$, и так как $\lim G_{i}$ дискретен, то $g_{n_{i}}=g$ и $h=h_{n_{i}}$ для достаточно большого $i$.

(c) Пусть $L_{i}-$ стабильный образ групп $G_{j}, j>i$, в группе $G_{i}$. Тогда $\lim _{\longleftarrow} G_{j}$ отображается на $L_{i}$. По условию существует $k$ такое, что $\lim G_{j} \rightarrow L_{i}$ биективно

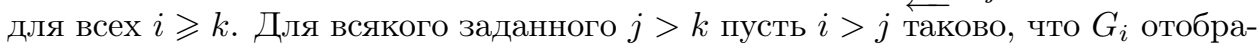
жается в $L_{j}$. Поскольку $L_{j} \rightarrow L_{k}$ биективно, $\operatorname{ker}\left(G_{i} \rightarrow G_{j}\right)=\operatorname{ker}\left(G_{i} \rightarrow G_{k}\right)$.

ПреДЛОжеНИЕ 3.5. Пусть $X$ - компакт, и зафиксируем некоторое $m \geqslant 0$.

(а) $X$ - предел обратной последовательности $m$-связных полиэдров, если и только если $\pi_{n}(X)=0$ для всех $n<m u \pi_{m}(X)$ антидискретна.

(b) $X$ - предел обратной последовательности $(m+1)$-связных отображений между $m$-связными полиэдрами, если и только если $\pi_{n}(X)=0$ для всех $n \leqslant m$.

Напомним, что отображение $f: X \rightarrow Y$ называется $m$-связным, если его цилиндр $M C(f)$ относительно $m$-связен, т. е. $\pi_{i}(M C(f), X)=0$ при $i \leqslant m$. Из точной гомотопической последовательности пары следует, что отображение 
$f: X \rightarrow Y$ между $m$-связными полиэдрами $(m+1)$-связно, если и только если $\pi_{m+1}(X) \rightarrow \pi_{m+1}(Y)-$ сюръекция.

Компакты, удовлетворяющие любому из эквивалентных условий предложения $3.5(\mathrm{a})$, именуются в литературе $\mathrm{UV}_{m}$-компактами или аппроксимативно $m$-связными или же $m$-щейпово связными. Это понятие фигурирует уже в [32; § 5], наряду с импликациями “только если” обеих частей предложения 3.5. Часть (а) хорошо известна (см., например, [29; доказательство 8.3.2]).

ДокАзАтельство. (а) Часть “только если" очевидна. Обратно, предположим, что $X$ - предел обратной последовательности полиэдров $P_{i}$. По теореме $3.1(\mathrm{~b}), \lim \pi_{n}\left(P_{i}\right)=0=\lim ^{1} \pi_{n}\left(P_{i}\right)$ при $n \leqslant m$. По леммам 3.3 и $3.4(\mathrm{a})$, для

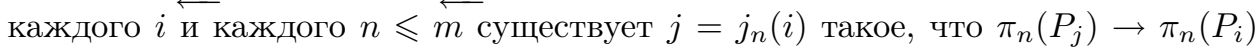
тривиально. Если $j=j_{0}\left(j_{1}\left(\ldots j_{k}\left(P_{i}\right)\right)\right)$, то, применяя индукцию по $k$, получим, что ограничение отображения $P_{j} \rightarrow P_{i}$ на $k$-мерный остов $P_{j}^{(k)}$ некоторой фиксированной триангуляции полиэдра $P_{j}$ нульгомотопно. Положим $i_{0}=0$ и $i_{k+1}=j_{0}\left(j_{1}\left(\ldots j_{m}\left(i_{k}\right)\right)\right)$, и пусть $Q_{k}$ - объединение $P_{i_{k}}$ с конусом $C P_{i_{k}}^{(m)}$. Тогда связующее отображение $p_{i_{k}}^{i_{k+1}}: P_{i_{(k+1)}} \rightarrow P_{i_{k}}$ продолжается до отображения $Q_{k+1} \rightarrow P_{i_{k}}$. Значит, $X-$ предел обратной последовательности отображений $Q_{k+1} \rightarrow P_{i_{k}} \subset Q_{k}$.

(b) Часть “только если" следует из теоремы 3.1 (b). Обратно, используя (a) и лемму 3.3 соответственно, предположим, что $X$ - предел обратной последовательности $m$-связных полиэдров $P_{i}$ и $G_{i}:=\pi_{m+1}\left(P_{i}\right)$ удовлетворяет условию Миттаг-Леффлера. Таким образом, для каждого $i$ существует $j(i)>i$ такое, что для каждого $k>j$ образ $p_{i}^{k}: G_{k} \rightarrow G_{i}$ равен образу $p_{i}^{j}$. Группа $G_{j}$ конечно порождена - в силу теоремы Гуревича, если $m>0$. Пусть $g_{1}, \ldots, g_{r}-$ множество порождающих элементов. Тогда $p_{i}^{j}\left(g_{l}\right)=p_{i}^{j+1}\left(h_{l}\right)$ для некоторых $h_{1}, \ldots, h_{r} \in G_{j+1}$. Пусть $f_{l}:\left(S^{m+1}, \mathrm{pt}\right) \rightarrow\left(P_{j}, p_{j}\right)-$ сфероид, представляющий $g_{l}^{\prime}=g_{l}^{-1} p_{i}^{i+1}\left(h_{l}\right)$, и пусть $Q_{j}$ получено склеиванием $f_{1}, \ldots, f_{r}$ по $(m+2)$-клеткам. Тогда $P_{j} \rightarrow P_{i}$ продолжается до отображения $Q_{j} \rightarrow P_{i}$, и композиция $P_{j+1} \rightarrow$ $P_{j} \subset Q_{j}$ индуцирует эпиморфизм на $\pi_{m+1}$. Полагая $i_{0}=0$ и $i_{n+1}=j\left(i_{n}\right)$, имеем $X=\underset{\lim }{\longleftarrow} Q_{i_{n}}$, где каждое $Q_{i_{n}} m$-связно и каждое связующее отображение $Q_{i_{n+1}} \rightarrow \overleftarrow{Q_{i_{n}}}(m+1)$-связно.

ЗАмЕЧАНИЕ. Хорошо известный пример, опирающийся на один глубокий результат Дж. Ф. Адамса, показывает, что предложение 3.5 не обобщается на случай $m=\infty$, т. е. существует бесконечномерный компакт $X$, не являющийся клеточным, для которого $\pi_{n}(X)=0$ при всех $n$ (см. [22; пример (5.5.10)], [29; пример (10.3.1)]).

Следующая “теорема Уайтхеда для стинродовских гомотопий” отвечает на вопрос Коямы.

ТеОрема 3.6. Пусть $X, Y$ - связные компакты размерностей $\leqslant m u \leqslant$ $m+1$ соответственно, и рассмотрим стинродовский гомотопический класс $f:(X, x) \rightsquigarrow(Y, y)$. Если $f_{*}: \pi_{n}(X) \rightarrow \pi_{n}(Y)$ - биекция при $n \leqslant m$ и сюргекиия при $n=m+1$, то $f$ является стинродовской гомотопической эквивалентностью. 
Так же, как и в случае классической теоремы Уайтхеда, доказательство теоремы 3.6 приводит к немного более общим утверждениям (a) и (b) ниже; добавление (c) следует из самой теоремы 3.6 вместе с последним утверждением добавления (b).

ДОБАВЛЕНИЯ к ТЕОРЕМЕ 3.6. Пусть $X$ u $Y$ - связные компакты $u f$ : $(X, x) \rightsquigarrow(Y, y)$ - стинродовский гомотопический класс.

(a) Eсли $\operatorname{dim} X \leqslant m, \operatorname{dim} Y \leqslant m+1$ u $i: Y \hookrightarrow Z-$ включение в связный компакт $Z$ такое, что композичия $\pi_{n}(X) \stackrel{f_{*}}{\longrightarrow} \pi_{n}(Y) \stackrel{i_{*}}{\longrightarrow} \pi_{n}(Z)$ является биекцией при $n \leqslant m$ и сюргекцией при $n=m+1$, то существует $g: Y \rightsquigarrow X$ такой, что $g f=\left[\mathrm{id}_{X}\right] u[i] f g=[i]$. В частности, $f_{*}: \pi_{n}(X) \rightarrow \pi_{n}(Y)$ есть расщепляющаяся ингекция для каждого $n$.

(b) Eсли $\operatorname{dim} Y \leqslant m+1$ u $f_{*}: \pi_{n}(X) \rightarrow \pi_{n}(Y)$ - биекиия при $n \leqslant m$ и сюроекиия при $n=m+1$, то существует $g: Y \rightsquigarrow X$ такой, что $f g=\left[\mathrm{id}_{Y}\right]$; и если $i: Z \hookrightarrow X$ - включение связного компакта $Z$ размерности $\leqslant m$, то $g$ может быть выбрано так, чтобы дополнительно $g f[i]=[i]$. В частности, $f_{*}: \pi_{n}(X) \rightarrow \pi_{n}(Y)$ есть расщепляющаяся сюргекция для каждого $n$.

(c) Если $\operatorname{dim} X \leqslant m, \operatorname{dim} Y \leqslant m u f_{*}: \pi_{n}(X) \rightarrow \pi_{n}(Y)-$ биекция при всех $n \leqslant m$, то $f$ является стинродовской гомотопической эквивалентностью.

Про-групповая версия теоремы 3.6 содержится в [22; теорема (5.5.6)] вместе с эскизом доказательства; другое доказательство дано в [23] (см. также [29] и [59; §4]). Кояма использовал эту версию для доказательства добавления (с) к теореме 3.6 при двух дополнительных предположениях: (i) $f_{*}: \check{\pi}_{n}(X) \rightarrow$ $\check{\pi}_{n}(Y)$ - биекция при $n \leqslant m$; (ii) техническое условие, которое немного слабее, чем требование, чтобы $Y$ было пределом обратной последовательности полиэдров с абелевыми фундаментальными группами [60]. Далее он спросил, не являются ли эти условия (i) и (ii) излишними [60; проблема 2]. Безнадежно ошибочное доказательство добавления (с) к теореме 3.6 приведено в [61], где без каких-либо пояснений $\pi_{1}(P, Q)$ считается группой для любой полиэдральной пары $(P, Q)$, а тривиальность " $\lim ^{1}$ " обратной последовательности таких

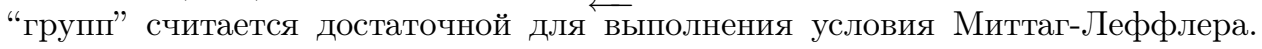
Корректная часть рассуждений, приведенных в [61], может быть расценена как доказательство того, что предположение (i) излишне в условии теоремы Коямы; однако никаких продвижений в вопросе о необходимости (ii) она не дает.

ДоказАТЕЛЬСтво теОРемЫ 3.6. Пусть $f_{[0, \infty)}: P_{[0, \infty)} \rightarrow Q_{[0, \infty)}$ представляет $f$. Без ограничения общности можно считать, что все $P_{i}$ и $Q_{i}$ связны. По лемме $2.5\left(\mathrm{~b}_{0}\right)$, после перехода к подпоследовательности $P_{i}$, мы можем предполагать, что $f_{[0, \infty)}$ сохраняет уровни. Для любого данного $J \subset$ $[0, \infty)$ пусть $f_{J}$ обозначает ограничение $P_{J} \rightarrow Q_{J}$ отображения $f$, а $M_{J}$ - цилиндр отображения $f_{J}$. Мы можем считать, что размерность $M_{[0, \infty)}$ не превышает $m+2$. Из условия и доказательства теоремы 3.1 (а) получаем, что $\pi_{n}\left(M_{[0, \infty)}, P_{[0, \infty)}\right)=0$ при $1 \leqslant n \leqslant m+1$. По доказательству теоремы $3.1(\mathrm{~b})$, имеем короткие точные последовательности

$$
0 \rightarrow \lim ^{1} \pi_{n+1}\left(M_{i}, P_{i}\right) \rightarrow \pi_{n}\left(M_{[0, \infty)}, P_{[0, \infty)}\right) \rightarrow \underset{\longleftarrow}{\lim } \pi_{n}\left(M_{i}, P_{i}\right) \rightarrow 0
$$


при $n \geqslant 1$, причем $\lim ^{1} \pi_{1}\left(P_{i}\right) \rightarrow \lim ^{1} \pi_{1}\left(M_{i}\right)$ сюръективно. Тогда $\pi_{n}\left(M_{i}, P_{i}\right)$ имеют тривиальный обратный предел при $1 \leqslant n \leqslant m+1$ и тривиальный производный предел при $2 \leqslant n \leqslant m+2$. По лемме 3.3 и лемме 3.7 ниже $\pi_{n}\left(M_{i}, P_{i}\right)$ удовлетворяет условию Миттаг-Леффлера при всех $1 \leqslant n \leqslant m+2$.

По лемме 3.4 (а) для любого $i$ найдется такое $j$, что $\pi_{n}\left(M_{j}, P_{j}\right) \rightarrow \pi_{n}\left(M_{i}, P_{i}\right)$ тривиально при $1 \leqslant n \leqslant m+1$. Переходя к подпоследовательности, мы можем предполагать, что $\pi_{n}\left(M_{i+1}, P_{i+1}\right) \rightarrow \pi_{n}\left(M_{i}, P_{i}\right)$ тривиально для $1 \leqslant n \leqslant m+1$. Мы также можем предполагать, что образ $\pi_{m+2}\left(M_{i+1}, P_{i+1}\right)$ в $\pi_{m+2}\left(M_{i}, P_{i}\right)$ равен образу $\pi_{m+2}\left(M_{j}, P_{j}\right)$ для всех $j>i$. Положим $i_{k}=(2 m+3) k$ и зафиксируем некоторую триангуляцию каждой пары $\left(M_{i_{k}}, P_{i_{k}}\right)$. Индукцией по $j=$ $0,1, \ldots, m+1$ устанавливается, что связующее отображение $p: M_{i_{k}} \rightarrow M_{i_{k}-j}$ гомотопно неподвижно на $P_{i_{k}}$ отображению $\varphi_{j}$, которое отправляет $j$-мерный остов $M_{i_{k}}^{(j)}$ в $P_{i_{k}-j}$. Таким образом, вводя обозначение $i_{k}^{\prime}=i_{k}-m-1$, получаем, что результирующее отображение $\psi_{k}:=\varphi_{m+1}$ отправляет $M_{i_{k}}$ в $P_{i_{k}^{\prime}}$, сужается до связующего отображения $p: P_{i_{k}} \rightarrow P_{i_{k}^{\prime}}$ и гомотопно связующему отображению $p: M_{i_{k}} \rightarrow M_{i_{k}^{\prime}}$ некоторой гомотопией $\Psi_{k}$.

Тогда $\mu: M_{i_{k+1}} \stackrel{p}{\longrightarrow} M_{i_{k}} \stackrel{\psi_{k}}{\longrightarrow} P_{i_{k}^{\prime}}$ и $\nu: M_{i_{k+1}} \stackrel{\psi_{k+1}}{\longrightarrow} P_{i_{k+1}^{\prime}} \stackrel{p}{\longrightarrow} P_{i_{k}^{\prime}}$ гомотопны со значениями в $M_{i_{k}^{\prime}}$ некоторой гомотопией $h_{k}$. Вводя обозначение $i_{k}^{\prime \prime}=$ $i_{k}^{\prime}-m-1=i_{k-1}+1$, получаем, как и выше, что композиции $\mu$ и $\nu$ со связующим отображением $P_{i_{k}^{\prime}} \rightarrow P_{i_{k}^{\prime \prime}}$ гомотопны некоторой гомотопией $h_{k}^{\prime}: M_{i_{k+1}} \times$ $I \rightarrow M_{i_{k}^{\prime \prime}}$, ограничение которой на $M_{i_{k}}^{(m)} \times I$ принимает значения в $P_{i_{k}^{\prime \prime}}$. Для каждого $(m+1)$-симплекса $\Delta$ триангуляции $M_{i_{k+1}}$ имеем $h_{k}^{\prime}(\partial(\Delta \times I)) \subset P_{i_{k}^{\prime \prime}}$. Пусть $\alpha_{\Delta}:(\Delta \times I, \partial) \rightarrow\left(M_{i_{k+1}^{\prime}}, P_{i_{k+1}^{\prime}}\right)$ - относительный сфероид, композиция которого со связующим отображением $M_{i_{k+1}} \rightarrow M_{i_{k-1}}$ гомотопна в $\left(M_{i_{k-1}}, P_{i_{k-1}}\right)$ композиции $\left.h_{k}^{\prime}\right|_{\Delta \times I}$ и связующего отображения $M_{i_{k}^{\prime \prime}} \rightarrow M_{i_{k-1}}$. Подправляя $\psi_{k+1}$ всеми $\alpha_{\Delta}$, мы получим, что композиции $\mu$ и подправленного $\nu$ со связующим отображением $P_{i_{k}^{\prime}} \rightarrow P_{i_{k-1}}$ гомотопны (со значениями в $P_{i_{k-1}}$ ) некоторой гомотопией $h_{k}^{\prime \prime}$. Таким образом, композиции $g_{k+1}: M_{i_{k+1}} \stackrel{\psi_{k+1}}{\longrightarrow} P_{i_{k+1}^{\prime}} \stackrel{p}{\longrightarrow} P_{i_{k-1}}$ являются целочисленными срезами сохраняющего уровни отображения $g_{[0, \infty)}: M_{[0, \infty)}^{i s} \rightarrow$ $P_{[0, \infty)}^{i}$, где $s(j)=j+2$ и $M_{j}^{i}=M_{i_{j}}$, ограничение которого на $P_{[0, \infty)}^{i s}$ совпадает со сдвигом $\left[p_{i_{0}}^{i_{2}}, p_{i_{\infty}}^{i_{\infty}}\right): P_{[0, \infty)}^{i s} \rightarrow P_{[0, \infty)}^{i}$.

По построению ${ }^{16} g_{[0, \infty)}$ собственно гомотопно $\left[p_{i_{0}}^{i_{2}}, p_{i_{\infty}}^{i_{\infty}}\right): M_{[0, \infty)}^{i s} \rightarrow M_{[0, \infty)}^{i}$. В самом деле, исправленное $\psi_{k+1}$ гомотопно себе исходному некоторой гомотопией $\Psi_{k+1}^{\prime}$ со значениями в $M_{i_{k+1}^{\prime}}$. Гомотопия $h_{k}^{\prime \prime}$ гомотопна композиции $h_{k}^{\prime}$ и связующего отображения $P_{i_{k}^{\prime \prime}} \rightarrow P_{i_{k-1}}$ посредством 2-гомотопии, ограниченной композициями $\Psi_{k}^{\prime}$ и $\Psi_{k+1}^{\prime}$ со связующими отображениями. В свою очередь, $h_{k}^{\prime}$ относительно гомотопна композиции $h_{k}$ и связующего отображения $P_{i_{k}^{\prime}} \rightarrow P_{i_{k}^{\prime \prime}}$. Наконец, $h_{k}$ гомотопна связующему отображению $M_{i_{k+1}} \rightarrow M_{i_{k}^{\prime}}$ посредством 2-гомотопии, ограниченной композициями $\Psi_{k}$ и $\Psi_{k+1}$ со связующими отображениями.

Лемма 3.7. Пусть $G_{i}$ - обратная последовательность групп и $H_{i}$ - обратная последовательность их подгрупп.

\footnotetext{
${ }^{16}$ или, иначе, ввиду импликации (iii) $\Rightarrow$ (i) из предложения 2.8 (b).
} 
(а) [54] Если обратная последовательность из пунктированных множеств $G_{i} / H_{i}$ правых смежных классов удовлетворяет условию Миттаг-Лефблера, то $\lim ^{1} H_{i} \rightarrow \lim ^{1} G_{i}$ сюоргективно.

(b) Если все $G_{i}$ счетны, то верно и обратное.

Поскольку доказательство леммы 3.3 уже использует и левое, и правое действия, кажется маловероятным, чтобы лемма 3.7 (b) могла быть аналогично доказана прямым подсчетом мощностей. Мы используем теорему Бэра о категории (см. следствие 7.11, в котором она выведена из бурбаковской “теоремы Миттаг-Леффлера").

Часть (а) пригодится немного позже (в доказательстве теоремы $3.15\left(\mathrm{~b}^{\prime}\right)$ ).

ДокАзАтельство. (а) Для разнообразия приведем набросок геометрического доказательства в конечнопредставленном случае. Пусть $\cdots \rightarrow\left(P_{2}, Q_{2}\right) \rightarrow$ $\left(P_{1}, Q_{1}\right)$ - обратная последовательность пар связных компактных полиэдров с $\pi_{1}\left(Q_{i}\right)=H_{i}$ и $\pi_{1}\left(P_{i}\right)=G_{i}$, причем включение $H_{i} \subset G_{i}$ индуцируется включением $Q_{i} \subset P_{i}$. Пусть $(X, A)=\underset{\lim }{\longleftarrow}\left(P_{i}, Q_{i}\right)$ и положим $S_{i}=\pi_{1}\left(P_{i}, Q_{i}\right)$. Предполагая, что $\left.\operatorname{im}\left[S_{i+1} \rightarrow S_{i}\right]=\operatorname{im} \overleftarrow{\left[S_{j}\right.} \rightarrow S_{i}\right]$ для всех $i$ и всех $j>i$, докажем, что $\pi_{0}(A) \rightarrow \pi_{0}(X)$ сюръективно. Представим любой наперед заданный элемент $\pi_{0}(X, b)$, где $b \in A$, собственным лучом $b_{[0,1]} \ell_{1} b_{[1,2]} \ell_{2} \ldots:[0, \infty) \rightarrow P_{[0, \infty)}$, где $b_{i}=p_{i}^{\infty}(b)$, a $\ell_{i}:(I, \partial I) \rightarrow\left(P_{i}, b_{i}\right)$ - петли. Тогда путь $b_{[0,1]} \ell_{1} b_{[1,2]}$ гомотопен со значениями в $\left(P_{[0,2]}, Q_{[0,2]} ; b_{2}\right)$ некоторому пути $\ell_{1}^{\prime}:(I, \partial I ;\{0\}) \rightarrow\left(P_{2}, Q_{2} ; b_{2}\right)$. Аналогично, $\ell_{1}^{\prime} \ell_{2} b_{[2,3]}$ гомотопен со значениями в $\left(P_{[1,3]}, Q_{[1,3]} ; b_{3}\right)$ некоторому пути $\ell_{2}^{\prime}:(I, \partial I ;\{0\}) \rightarrow\left(P_{3}, Q_{3} ; b_{3}\right)$. Комбинируя все такие гомотопии, получим гомотопию между исходным собственным лучом и некоторым собственным лучом со значениями в $Q_{[0, \infty]}$.

(b) Предположим, что $G_{i} / H_{i}$ не удовлетворяют условию Миттаг-Леффлера. Тогда существует $k$ такое, что образы $G_{k+i} / H_{k+i}$ в $G_{k} / H_{k}$ не стабилизируются. Заметим, что они суть то же самое, что и образы композиций $G_{k+i} \rightarrow$ $G_{k} \rightarrow G_{k} / H_{k}$. Пусть $A_{i}$ - образ $G_{k+i}$ в $G_{k}$, и пусть $B_{i}=A_{i} \cap H_{k}$. Тогда образы $A_{i}$ в $A_{0} / B_{0}$ не стабилизируются, равно как и прообразы $A_{i} B_{0}$ этих образов. Антиавтоморфизм $g \mapsto g^{-1}$ отправляет их в $B_{0} A_{i}$, откуда получаем, что каждое пунктированное множество $B_{0} \backslash A_{i} / A_{i+1}$ двойных смежных классов нетривиально. Каждое $G_{k+i} \rightarrow A_{i}$ сюръективно, поэтому $\lim ^{1} G_{k+i} \rightarrow \lim ^{1} A_{i}$ сюръективно. Композиция $\lim ^{1} H_{k+i} \rightarrow \lim ^{1} G_{k+i} \rightarrow \lim ^{1} A_{i}$ пропускается через $\lim ^{1} B_{i}$, следовательно, $\lim ^{1} \overleftarrow{B_{i}} \rightarrow \lim ^{1} A_{i}$ сюръективно. Теперь доказательство

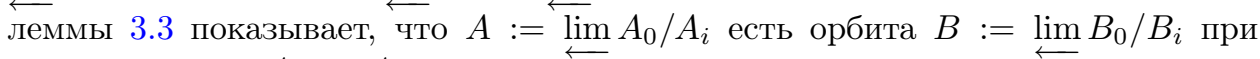
левом действии $A_{0}$ на $A$.

Топология обратного предела на $A$ индуцирована ультраметрикой $d$, определенной как $d(x, y)=1 / n$, если $p_{n}^{\infty}(x)=p_{n}^{\infty}(y) \in A_{0} / A_{n}$, но $p_{n+1}^{\infty}(x) \neq p_{n+1}^{\infty}(y) \in$ $A_{0} / A_{n+1}$. Очевидно, что $A$ полно в этой метрике (в сущности, оно является пополнением $\left.A_{0} / \cap A_{i}\right)$. Шар радиуса $1 / n$ является прообразом точки при отображении $p_{n}^{\infty}$. Если такой шар $\left(p_{n}^{\infty}\right)^{-1}\left(g A_{n}\right)$ пересекает $B$, то $g A_{n}$ содержит некоторый $b \in B_{0}$. В этом случае пусть $B_{0} a A_{n+1}-$ какой-нибудь нетривиальный элемент $B_{0} \backslash A_{n} / A_{n+1}$. Тогда $b a A_{n+1}$ содержится в $g A_{n}$ и не пересекает $B_{0}$. Поэтому $\left(p_{n+1}^{\infty}\right)^{-1}\left(b a A_{n+1}\right)$ содержится в $\left(p_{n}^{\infty}\right)^{-1}\left(g A_{n}\right)$ и не пересекает $B$. Мы 
доказали, что $B$ нигде не плотно в $A$. Следовательно, по теореме Бэра о категории, $A$ не может быть объединением счетного количества сдвигов $g B$ множества $B$ при левом действии $A_{0}$ на $A$ изометриями. Противоречие. Лемма 3.7 доказана.

Понтрягин рассматривал отношение эквивалентности на обратных последовательностях групп, порожденное операцией перехода к подпоследовательности [35; гл. III, § I]. Аналогично доказательству предложения 2.6, (ii) $\Leftrightarrow$ (iii), две обратные последовательности $G_{i}, H_{i}$ абелевых групп эквивалентны в смысле Понтрягина тогда и только тогда, когда они связаны про-изоморбизмом, который является набором отображений $f_{i}: G_{i} \rightarrow H_{i}$, коммутирующих со связующими отображениями, таким, что существуют возрастающая последовательность $k: \mathbb{N} \rightarrow \mathbb{N}$ и гомоморфизмы $g_{i}: H_{k(i+1)} \rightarrow G_{k(i)}$ такие, что композиции $g_{i} f_{k(i+1)}: G_{k(i+1)} \rightarrow G_{k(i)}$ и $f_{k(i)} g_{i}: H_{k(i+1)} \rightarrow H_{k(i)}$ равняются связующим отображениям.

ТЕОрема 3.8. Набор гомоморфизмов $f_{i}: G_{i} \rightarrow H_{i}$ между обратными последовательностями счетных групп, коммутирующих со связующими отображениями, является про-изоморбизмом, если и только если $\lim _{i} f_{i}: \lim _{\leftarrow} G_{i} \rightarrow$ $\underset{\lim }{\longleftarrow} H_{i}$ u $\lim ^{1} f_{i}: \lim ^{1} G_{i} \rightarrow \lim ^{1} H_{i}$ суть биеки,и

Этим дается ответ на вопрос Коямы [60; проблема 1], установившего частный случай, в котором каждая $f_{i}\left(G_{i}\right)$ нормальна в $H_{i}[60 ;$ лемма 2]. Конечнопорожденный абелев случай был вначале анонсирован Кислингом [62; теорема 2.4].

Оставшаяся часть доказательства теоремы 3.8 (за исключением леммы 3.7) аналогична доказательству [60; лемма 2], но ввиду того, что чтение последнего осложнено опечаткой и несколькими дальнейшими ссылками, для удобства приведем подробности.

ДоКАЗАТЕЛьСтво. Часть “только если” следует из того, что для всякой обратной последовательности групп $\Gamma_{i}$ связующие отображения индуцируют биекции $\lim \Gamma_{i+1} \rightarrow \underset{\lim }{ } \Gamma_{i}$ и $\lim ^{1} \Gamma_{i+1} \rightarrow \lim ^{1} \Gamma_{i}$.

Обрат $\overleftarrow{н о}$, каждое $\overleftarrow{f_{i}}$ доставляет короткие точные последовательности $1 \rightarrow$ $K_{i} \rightarrow G_{i} \rightarrow f_{i}\left(G_{i}\right) \rightarrow 1$ и $1 \rightarrow f_{i}\left(G_{i}\right) \rightarrow H_{i} \rightarrow L_{i} \rightarrow$ *, где $K_{i}=\operatorname{ker} f_{i}$ и $L_{i}-$ пунктированные множества $H_{i} / f\left(G_{i}\right)$ правых смежных классов. Из условия и шестичленной точной последовательности в теореме 3.1 (части $(\mathrm{d})$ и $\left(\mathrm{d}^{\prime}\right)$ ) вытекает, что $\lim _{\longleftarrow} K_{i}=\lim ^{1} K_{i}=1$ и $\lim _{\longleftarrow} L_{i}=*$. По лемме 3.3 условию МиттагЛеффлера удовлетворяет $K_{i}$, а по лемме 3.7 также и $L_{i}$. Пусть $k(0)=0$, и предположим, что $k(i)$ определено. Тогда по лемме 3.4 (а) существуют $j>k(i)$ и $k(i+1)>j$ такие, что $L_{k(i+1)} \rightarrow L_{j}$ и $K_{j} \rightarrow K_{k(i)}$ тривиальны. Для всякого наперед заданного $h \in H_{k(i+1)}$ его образ в $H_{j}$ равен $f_{j}\left(g_{j}\right)$ для некоторого $g_{j} \in G_{j}$. Для всякого наперед заданного $g_{j}^{\prime} \in G_{j}$ с $f_{j}\left(g_{j}^{\prime}\right)=f_{j}\left(g_{j}\right)$ его образ $g \in G_{i}$ равен образу $g_{j}$. Следовательно, $g_{i}: H_{k(i+1)} \rightarrow G_{k(i)}, h \mapsto g$, определено корректно. Непосредственная проверка показывает, что это гомоморфизм и что $g_{i}$ обращают $h_{i}$, как и требовалось.

Теорема 3.8 доказана.

Упомянем два известных следствия теоремы 3.6; третье появится в теореме 3.15 (e). 
Следующий результат был доказан в [23], а также следует из $\pi_{\infty}$-критерия Зибенмана [63], равно как и из [22; теорема (5.5.6)].

Теорема 3.9. Пусть $X$ и $Y$ - связные конечномерные компакты. Стинродовский гомотопический класс $f:(X, x) \rightsquigarrow(Y, y)$ является стинродовской гомотопической эквивалентностью, если и только если он представлен сохраняющим уровни отображением $f_{[0, \infty)}:\left(P_{[0, \infty)}, p_{[0, \infty)}\right) \rightarrow\left(Q_{[0, \infty)}, q_{[0, \infty)}\right)$, являющимся изоморбизмом в про-гомотопиях.

Определение изоморфизма в про-гомотопиях см. в формулировке предложения 2.6. Интересное альтернативное доказательство теоремы 3.9 дано в [48].

ДокАЗАтельство. Часть “только если” следует из леммы $2.5\left(\mathrm{~b}_{0}\right),\left(\mathrm{b}_{1}\right)$. Обратно, индуцированные отображения $\left(f_{i}\right)_{*}: \pi_{n}\left(P_{i}, p_{i}\right) \rightarrow \pi_{n}\left(Q_{i}, q_{i}\right)$ доставляют изоморфизм про-групп для каждого $n$. Поэтому, в силу части "только если" теоремы 3.8 и теоремы $3.1(\mathrm{~b}), f_{*}: \pi_{n}(X) \rightarrow \pi_{n}(Y)$ является изоморфизмом для всех $n$. Следовательно, по теореме $3.6 f$ есть стинродовская гомотопическая эквивалентность.

Теорема 3.9 доказана.

Если $P$ - некомпактный полиэдр, то $\pi_{n}(P):=\left[\left(S^{n}, \mathrm{pt}\right),(P, \mathrm{pt})\right]$ изоморфна $\stackrel{\Delta}{\pi}_{n}(P)$ по предложению $2.3(\mathrm{a})$.

ТеОрема 3.10 (Эдвардс-Гэган). Пусть $X-m$-мерный связный компакт. Следующие утверждения равносильны:

(i) $\pi_{n}(X)$ дискретна для всех $n \leqslant m$;

(ii) $X$ пунктированно стинродовски гомотопически доминируется некоторым компактным полиэдром;

(iii) $X$ допускает отображение в полиэдр, индуцирующее изоморбизмы на $\pi_{n}$ для всех $n \leqslant m$;

(i') $\pi_{n}(X)$ дискретна для всех $n$;

(ii') $X$ пунктированно стинродовски гомотопически доминируется некоторым компактным т-полиэдром;

(iii') $X$ допускает отображение в $(m+1)$-полиэдр, индуцирующее изоморфизмына $\pi_{n}$ для всех $n$.

В литературе имеется немало различных доказательств теоремы 3.10. Правда, многие из них весьма туманны с геометрической точки зрения: в оригинальном доказательстве в работах [64], [65] (см. также упрощение в [66]) используются несепарабельные полиэдры; Дыдак [67; 7.4] заменяет связующие отображения серровскими расслоениями; Ферри [21; теорема 4 и первый абзац ее же доказательства] работает с $Q$-многообразиями $(Q$ - гильбертов куб). Замкнутое геометрическое доказательство можно извлечь из статей Кодамы [20], [68], о чем автор настоящей статьи узнал лишь после того, как были записаны оба нижеприведенных доказательства. Второе из них, в действительности, можно считать упрощенной версией рассуждения Кодамы.

ДокАзАтельство. (i) $\Rightarrow$ (ii). Эта импликация будет доказана комбинацией добавления (а) к теореме 3.6 с приемом из доказательства предложения 3.5 (b). Ниже будет дано другое доказательство, опирающееся на теорему 3.12 . 
Предположим, что $X$ - предел обратной последовательности компактных $m$-полиэдров $P_{i}$. Так как группа $\pi_{n}(X)$ хаусдорфова для каждого $n \leqslant m$, по теореме $3.1(\mathrm{~b})$ и лемме 3.3 обратная последовательность $\pi_{n}\left(P_{i}\right)$ удовлетворяет условию Миттаг-Леффлера для каждого $n \leqslant m+1$. Применяя переиндексацию, мы можем считать, что для каждого $i$ и каждого $n \leqslant m+1$ образ $G_{i}^{[n]}$ группы $\pi_{n}\left(P_{i+1}\right)$ в $\pi_{n}\left(P_{i}\right)$ равен образу каждой $\pi_{n}\left(P_{j}\right)$ с $j>i$. Тогда каждая $G_{i+1}^{[n]}$ сюръектируется на $G_{i}^{[n]}$ и на нее же сюръектируется $\pi_{n}(X)$ для каждого $n \leqslant m+1$.

Так как $\pi_{n}(X)$ дискретна при $n \leqslant m$, то по лемме $3.4(\mathrm{~b})$ она инъектируется в $\pi_{n}\left(P_{k}\right)$ для некоторого $k$, которое можно считать одинаковым для всех $n \leqslant m$. Положим $q=k+m+1$. Так как $G_{q}^{[1]}$ сюръектируется на $G_{q-1}^{[1]}$, то каждое $x \in \pi_{1}\left(P_{q}\right)$ имеет тот же образ в $\pi_{1}\left(P_{q-1}\right)$, что и некоторый $y_{x} \in G_{q}^{[1]}$. Приклеивая 2 -клетку к $P_{q}$ по некоторой представляющей петле элемента $x y_{x}^{-1}$ для каждого $x \in \pi_{1}\left(P_{q}\right)$, мы получаем (быть может, некомпактный) полиэдр $P_{q}^{[1]}$ такой, что связующее отображение $P_{q} \rightarrow P_{q-1}$ пропускается через включение $P_{q} \subset P_{q}^{[1]}$ и $\pi_{1}(X)$ отображается на $\pi_{1}\left(P_{q}^{[1]}\right)$. Если клетки приклеивать к $P_{q} \times[0, \infty)$, а не к $P_{q}$, то легко обеспечить локальную компактность результирующего полиэдра. Далее, поскольку $\pi_{2}\left(P_{q-1}\right)$ отображается в $G_{q-2}^{[2]}$, туда же идет и $\pi_{2}\left(P_{q}^{[1]}\right)$. Следовательно, каждый $x \in \pi_{2}\left(P_{q}^{[1]}\right)$ имеет тот же образ в $\pi_{2}\left(P_{q-2}\right)$, что и некоторый элемент $y_{x}$ образа $G_{q}^{[2]}$ в $\pi_{1}\left(P_{q}\right)$. Продолжая процесс, мы, в конечном счете, пропустим связующее отображение $P_{q} \rightarrow P_{k}$ через последовательность включений $P_{q} \subset P_{q}^{[1]} \subset \cdots \subset P_{q}^{[m+1]}=: Q$ таких, что $\pi_{n}(X)$ отображается на $\pi_{n}(Q)$ для каждого $n \leqslant m+1$.

Так как группа $\pi_{n}(X)$ инъектируется в $\pi_{n}\left(P_{k}\right)$ для всех $n \leqslant m$, она инъектируется и в $\pi_{n}(Q)$ для всех $n \leqslant m$. Таким образом, тройка $X \rightarrow P_{q} \rightarrow Q$ удовлетворяет условию добавления (а) к теореме 3.6 , за исключением того, что $Q$ не обязательно компактен. Тем не менее, доказательство добавления (а) применимо и показывает, что $P_{q}$ пунктированно стинродовски гомотопически доминирует $X$.

(ii) $\Rightarrow$ (iii) и $\left(\right.$ ii' $\left.^{\prime}\right) \Rightarrow\left(\right.$ iii' $\left.^{\prime}\right)$. Если $K-$ компактный полиэдр и $d:(K, b) \rightsquigarrow(X, x)$ и $u:(X, x) \rightsquigarrow(K, b)$ таковы, что $d u=\left[\mathrm{id}_{X}\right]$, то рассмотрим дважды бесконечный телескоп $P:=\operatorname{Tel}(\cdots \stackrel{q}{\rightarrow} K \stackrel{q}{\rightarrow} K \stackrel{q}{\rightarrow} \cdots)$, где $q:(K, b) \rightarrow(K, b)-$ кусочно линейное отображение, представляющее стинродовский гомотопический класс $u d$ в силу предложения 2.3 (а). Из приема Мезера (см. [69]) и предложения 2.3 (а) получается собственное отображение $X \times \mathbb{R} \rightarrow P$ такое, что композиция $X \times\{0\} \subset X \times \mathbb{R} \rightarrow P$ индуцирует изоморфизм на всех $\pi_{n}$.

(i) $\Rightarrow\left(\right.$ i' $^{\prime}$, (ii) $\Rightarrow\left(\right.$ ii' $\left.^{\prime}\right)$, (iii) $\Rightarrow($ i $), \quad\left(\right.$ iii $\left.^{\prime}\right) \Rightarrow\left(\right.$ i' $\left.^{\prime}\right)$. Очевидны.

ЗАмЕчАНИЕ. Как подмечено в [64], компакты, удовлетворяющие равносильным условиям теоремы 3.10, не обязаны иметь шейп компактного полиэдра. Действительно, пусть $P$ - полиэдр, гомотопически доминированный компактным полиэдром $K$, но не гомотопически эквивалентный никакому компактному полиэдру (см. [69]). Если $d: K \rightarrow P$ и $u: P \rightarrow K$ таковы, что $d u \simeq \mathrm{id}_{P}$, то предел $X$ обратной последовательности $\widehat{K}:=(\cdots \stackrel{u d}{\longrightarrow} K \stackrel{u d}{\longrightarrow} K)$ таков, что проекция $f: X \rightarrow P$ индуцирует изоморфизм на стинродовских гомотопических 
группах. Действительно, нетрудно видеть, что сохраняющие уровни отображения $d_{[0, \infty)}: \widehat{K}_{[0, \infty)} \rightarrow P \times[0, \infty)$ и $u_{[0, \infty)}: P \times[0, \infty) \rightarrow \widehat{K}_{[0, \infty)}$, определенные соответственно с помощью $d$ и $u$ на целочисленных уровнях и продолженные на все уровни с использованием гомотопий $d \simeq d u d$ и $u d u \simeq u$, взаимно обратны в полусобственных гомотопиях. Если же существует стинродовская гомотопическая эквивалентность $g: L \rightsquigarrow X$ для некоторого компактного полиэдра $L$, то $[f] g: L \rightsquigarrow P$ представи́мо отображением $h: L \rightarrow P$ по предложению 2.3 (а). Так как $h$ индуцирует изоморфизм гомотопических групп, оно является гомотопической эквивалентностью в силу классической теоремы Уайтхеда, что противоречит сделанному предположению.

ЗАмечАниЕ. Ферри доказал, допуская возможность $m=\infty$, что для связного компакта $X$ группа $\pi_{n}(X)$ дискретна при всех $n<m$, если и только если $X$ имеет шейп компакта, являющегося локально $n$-связным при всех $n<m$ [21]. Случай $m=1$ был изначально доказан Красинкевичем (см. [29]); по поводу части "если" см. §6. В частности, следующие утверждения могут быть добавлены к списку теоремы 3.10:

(iv) $X$ имеет шейп локально $m$-связного компакта;

$\left(\mathrm{iv}^{\prime}\right) X$ имеет шейп компакта, удовлетворяющего условию $\mathrm{LC}_{\infty}$.

Лемма 3.11 (Дыдак). Пусть $A_{i} \rightarrow B_{i} \rightarrow C_{i} \rightarrow D_{i}-$ точная последовательность обратных последовательностей групп - или, более общо, пунктированных множеств, где каждое $A_{i}$ - группа, действующая на $B_{i}$ так, что непустые прообразы точек отображения $B_{i} \rightarrow C_{i}$ являются в точности орбитами этого действия.

Если $A_{i}$ и $C_{i}$ удовлетворяют условиям Миттаг-Леффлера и $D_{i}$ удовлетворяют двойственному условию Миттаг-Лефблера, то $B_{i}$ удовлетворяют условию Миттаг-Лефблера.

Лемма 3.11 была доказана в [70], где она сформулирована в ослабленной форме (недостаточной для ее применения ниже). В $\S 8$ нам понадобится не только сама лемма 3.11, но и ее доказательство, поэтому для удобства мы воспроизведем его, стремясь к относительной читабельности.

ДокАЗАТЕЛЬСтво. Проведя перенумерацию этажей, мы можем предполагать, что $\operatorname{im}\left(A_{i+1} \rightarrow A_{i}\right)=\operatorname{im}\left(A_{i+2} \rightarrow A_{i}\right), \operatorname{im}\left(C_{i+1} \rightarrow C_{i}\right)=\operatorname{im}\left(C_{i+2} \rightarrow C_{i}\right)$ и $\operatorname{ker}\left(D_{i} \rightarrow D_{i-1}\right)=\operatorname{ker}\left(D_{i} \rightarrow D_{i-2}\right)$ для каждого $i$. Достаточно доказать, что $\operatorname{im}\left(B_{n+2} \rightarrow B_{n}\right)=\operatorname{im}\left(B_{n+3} \rightarrow B_{n}\right)$ для каждого $n$.

Итак, пусть $b_{n}$ - образ некоторого $b_{n+2} \in B_{n+2}$. Так как $\operatorname{im}\left(C_{n+2} \rightarrow C_{n+1}\right)=$ $\operatorname{im}\left(C_{n+4} \rightarrow C_{n+1}\right)$, то образ $b_{n+2}$ в $C_{n+1}$ является образом некоторого $c_{n+4} \in$ $C_{n+4}$. Тогда образ $c_{n+4}$ в $D_{n+1}$ равен образу $b_{n+2}$ в $D_{n+1}$, который тривиален в силу точности строк. Поскольку $\operatorname{ker}\left(D_{n+4} \rightarrow D_{n+1}\right)=\operatorname{ker}\left(D_{n+4} \rightarrow D_{n+3}\right)$, образ $c_{n+4}$ должен быть тривиален уже в $D_{n+3}$. Значит, образ $c_{n+4}$ в $C_{n+3}$ является образом некоторого $b_{n+3} \in B_{n+3}$. По построению $b_{n+2}$ и $b_{n+3}$ отображаются в один и тот же элемент в $C_{n+1}$. Следовательно, их образы в $B_{n+1}$ связаны действием некоторого $a_{n+1} \in A_{n+1}$. Так как $\operatorname{im}\left(A_{n+1} \rightarrow A_{n}\right)=\operatorname{im}\left(A_{n+3} \rightarrow A_{n}\right)$, то образ $a_{n+1}$ в $A_{n}$ является образом некоторого $a_{n+3} \in A_{n+3}$. Наконец, результат действия $a_{n+3}$ на $b_{n+3}$ отображается на $b_{n}$. Лемма 3.11 доказана. 
ЗАмечАниЕ. Вышеприведенное рассуждение показывает, что для фиксированного $n$ : (i) если образы $A_{i}$ в $A_{n}$ стабилизируются, $C_{i}$ удовлетворяют условию Миттаг-Леффлера, а $D_{i}$ удовлетворяют двойственному условию Миттаг-Леффлера, то образы $B_{i}$ в $B_{n}$ стабилизируются; (ii) если образы $C_{i}$ в $C_{n}$ стабилизируются, каждое отображение $A_{i+1} \rightarrow A_{i}$ является изоморфизмом и $D_{i}$ удовлетворяют двойственному условию Миттаг-Леффлера, то образы $B_{i}$ в $B_{n}$ стабилизируются. Это будет использовано в $\S 8$.

Если $P$ - локально компактный полиэдр, то скажем, что $P$ собственно $m$-связен на бесконечности, если всякое компактное подмножество $Q$ полиэдра $P$ содержится в таком компактном подмножестве $R$ полиэдра $P$, что всякое собственное отображение $\mathbb{R}^{n} \rightarrow \mathrm{Cl}(P \backslash R)$ при условии $n \leqslant m$ продолжается до собственного отображения $\mathbb{R}^{n} \times[0, \infty) \rightarrow \mathrm{Cl}(P \backslash Q)$. Ясно, что это условие влечет локальную $n$-связность одноточечной компактификации полиэдра $P$. Обратная импликация не имеет места (см. теорему 6.12 (а) ниже).

ТеОрема 3.12 (Кодама). Пусть $X$ - предел обратной последовательности компактных полиэдров $P_{i}$, и зафиксируем некоторое $m \geqslant 0$. Тогда $\pi_{n}(X)$ дискретна для всех $n<m$, если и только если $P_{[0, \infty)}$ собственно $m$-связен на бесконечности.

Теорема 3.12 является по существу переформулировкой некоторых результатов работ [20], [68]. Рассуждения Кодамы заметно упрощены ниже благодаря использованию лемм 3.11 и 3.13. Гомологический аналог теоремы 3.12 был получен Дыдаком (см. теорему $6.12(\mathrm{~b}))$.

ДокАЗАТЕЛЬство. Мы рассмотрим лишь случай $m>0$. Теорема 3.1 (а) доставляет следующую точную последовательность обратных последовательностей:

$$
\pi_{n}(X) \rightarrow \pi_{n}\left(P_{[k, \infty]}\right) \rightarrow \pi_{n}\left(P_{[k, \infty]}, X\right) \rightarrow \pi_{n-1}(X) \rightarrow \pi_{n-1}\left(P_{[k, \infty]}\right) .
$$

Легко видеть, что $P_{[k, \infty]}$ стинродовски деформационно ретрагируется на $P_{k}$, так что их стинродовские гомотопические группы (пунктированные множества при $n=0)$ можно отождествить.

Если $\pi_{n}(X)$ дискретна для каждого $n<m$, то по теореме 3.1 (b) и лемме 3.3 группы $\pi_{n}\left(P_{k}\right)$ удовлетворяют условию Миттаг-Леффлера для каждого $n \leqslant m$. По лемме $3.4($ с $)$ группы $\pi_{n}\left(P_{k}\right)$ удовлетворяют двойственному условию Миттаг-Леффлера для каждого $n<m$. Значит, по лемме 3.11 группы $\pi_{n}\left(P_{[k, \infty]}, X\right)$ удовлетворяют условию Миттаг-Леффлера для каждого $n \leqslant m$. С другой стороны, по теореме 3.1 (c) $\left.\lim _{n} \pi_{[k, \infty]}, X\right)$ изоморфен группе $\pi_{n}(X, X)$, которая тривиальна. Тогда по лемме 3.4 (а) для каждого $i$ существует $j>i$ такое, что отображение $\pi_{n}\left(P_{[j, \infty]}, X\right) \rightarrow \pi_{n}\left(P_{[i, \infty]}, X\right)$ тривиально для каждого $n \leqslant m$.

Обратно, предположим, что для каждого $i$ существует $j>i$ такое, что $\pi_{n}\left(P_{[j, \infty]}, X\right) \rightarrow \pi_{n}\left(P_{[i, \infty]}, X\right)$ тривиально. Тогда $\pi_{n-1}(X) \rightarrow \pi_{n-1}\left(P_{j}\right)$ инъективно согласно вышеприведенной точной последовательности. Значит, $\pi_{n-1}(X)$ дискретна.

Для завершения доказательства используем лемму 3.13 ниже. Ясно, что сохраняющее базисный луч собственное отображение $\mathbb{R}^{n} \rightarrow P_{[k, \infty)}$ продолжа- 
ется до собственного отображения $\mathbb{R}^{n} \times[0, \infty) \rightarrow P_{[k, \infty)}$, если и только если оно "нульгомотопно" сохраняющей базисный луч собственной гомотопией. Более того, всякое собственное отображение $\mathbb{R}^{n} \rightarrow P_{[k, \infty)}$ собственно гомотопно такому, которое сохраняет базисный луч, если только всякое сохраняющее базисный луч отображение $\mathbb{R}^{1} \rightarrow P_{[k, \infty)}$ "нульгомотопно" сохраняющей базисный луч собственной гомотопией.

Лемма 3.13. Пусть $X$ - предел обратной последовательности компактньх полиэдров $P_{i}$. Имеется естественная пунктированная биекция между $\pi_{n}\left(P_{[k, \infty]}, X\right)$ и множеством сохраняющих базисный луч собственных отображсений $\mathbb{R}^{n} \rightarrow P_{[k, \infty)}$ с точностъю до сохраняющей базисный луч собственной гомотопии.

Обратная последовательность пунктированных множеств (групп при $n>1$ ) $\pi_{n}\left(P_{[k, \infty]}, X\right)$ рассматривалась Кодамой под названием "про-группа подвижности" [42]; точнее, Кодама определял их посредством утверждения леммы 3.13, очевидно не догадываясь, что они совпадают с относительными стинродовскими гомотопическими множествами (с которыми он был хорошо знаком).

ДокАзАТЕЛЬство. По определению $\pi_{n}\left(P_{[k, \infty]}, X\right)$ есть множество классов сохраняющих базисный луч собственных отображений

$$
\left(\mathbb{R}^{n} \times[0, \infty), \mathbb{R}^{n} \times\{0\} \backslash B^{n}\right) \rightarrow\left(P_{[k, \infty)} \times[0, \infty), P_{[k, \infty)} \times\{0\}\right)
$$

с точностью до сохраняющей базисный луч собственной гомотопии, где $B^{n} \subset$ $\mathbb{R}^{n} \times\{0\}-$ некоторый $n$-мерный шар (мы опускаем базисный луч в обозначениях). Поскольку $[0, \infty)$ деформационно ретрагируется на $\{0\}$, то, не ограничивая общности, мы можем предполагать, что $B^{n}$ все же переводится в $P_{[k, \infty)} \times\{0\}$ при всех рассматриваемых отображениях и гомотопиях. Отсюда получаем требуемое описание.

Из теоремы 3.12 немедленно вытекает следующее утверждение.

СледСтвиЕ 3.14 (Дыдак [71]). Если $X$ u $Y$ - компакты такие, что $\pi_{i}(X)$ $u \pi_{i}(Y)$ дискретны для всех $i \leqslant n, a \pi_{i}(X \cap Y)$ дискретны для всех $i \leqslant n-1$, то $\pi_{i}(X \cup Y)$ дискретны для всех $i \leqslant n$.

Доказательство Дыдака использует гомологии, универсальные накрытия и почти связные по Стинроду компакты (относительно последних см. теорему 6.12). В более сильном предположении о дискретности $\pi_{i}(X \cap Y)$ для всех $i \leqslant n$ следствие 3.14 было получено Кодамой [20], [68]. Случай $n=0$ был получен Красинкевичем (см. теорему 8.3 ниже).

ДРУГОЕ ДОКАЗАТЕЛЬСТВО ТЕОРЕМЫ 3.10. (i) $\left.\Rightarrow\left(\mathrm{ii}^{\prime}\right)\right)$. В терминологии $\S 6$ это будет стинродовской версией конструкции Ферри. А именно, мы построим стинродовскую ретракцию $P_{[k, \infty]} \rightsquigarrow X$ для некоторого $k$. Отсюда будет следовать, что $P_{k}$ стинродовски гомотопически доминирует $X$, поскольку легко видеть, что $P_{[k, \infty]}$ стинродовски деформационно ретрагируется на $P_{k}$.

Зафиксируем триангуляцию $P_{[0, \infty]}$, диаметры симплексов которой стремятся к нулю при приближении к $X$. Для некоторых заданных $n$ и $k_{n}$ введем обозначение $Q^{n}=\left(\cdots \rightarrow Q_{k_{n}+1}^{n} \rightarrow Q_{k_{n}}^{n}\right)$ для обратной последовательности $\cdots \rightarrow P_{\left[k_{n}, k_{n}+2\right]}^{(n)} \rightarrow P_{\left[k_{n}, k_{n}+1\right]}^{(n)} \rightarrow P_{k_{n}}^{(n)}$, пределом которой является $n$-мерный 
остов $P_{\left[k_{n}, \infty\right]}^{(n)}$. Тогда стинродовская ретракция $P_{\left[k_{n}, \infty\right]}^{(n)} \cup X \rightsquigarrow X-$ это собственный гомотопический класс собственной ретракции $r^{n}: Q_{[0, \infty)}^{n} \cup P_{[0, \infty)} \rightarrow P_{[0, \infty)}$. Поскольку $\pi_{n}(X)$ дискретна при всех $n<0$ (пустое условие), то по теореме 3.12 для каждого $i$ существует $j=j(i)>i$ такое, что каждая вершина $P_{[j, \infty)}$ есть начало собственного луча $[0, \infty) \rightarrow P_{[i, \infty)}$. Это доставляет $r^{0}$, причем с $k_{0}=j(0)$. Предположив, что $r^{n}$ уже определена, ретракцию $r^{n+1}$ возможно определить, поскольку по теореме 3.12 для каждого $i$ существует $j=j(i)>i$ такое, что всякое собственное отображение $f: S^{n} \times[0, \infty) \rightarrow P_{[j, \infty)}$ такое, что $f\left(S^{n} \times\{0\}\right)=\partial \Delta$ для некоторого $(n+1)$-симплекса $\Delta$ триангулированного полиэдра $P_{[j, \infty)}$, продолжается до собственного отображения $\bar{f}: B^{n+1} \times[0, \infty) \rightarrow P_{[i, \infty)}$ такого, что $\bar{f}\left(B^{n+1} \times\{0\}\right)=\Delta$. Наконец, $\left[r^{m}\right]: P_{[k, \infty]} \rightsquigarrow X-$ требуемая стинродовская ретракция.

Стинродовские расслоения. Пусть $P=\left(\cdots \stackrel{p_{1}}{\longrightarrow} P_{1} \stackrel{p_{0}}{\longrightarrow} P_{0}\right)$ и $Q=\left(\cdots \stackrel{q_{1}}{\longrightarrow}\right.$ $\left.Q_{1} \stackrel{q_{0}}{\longrightarrow} Q_{0}\right)$ - обратные последовательности компактных полиэдров и кусочно линейных отображений, и пусть $w: P \rightarrow Q-\mathrm{PL}-$ поэтажное отображение, т. е. последовательность кусочно линейных отображений $w_{i}: P_{i} \rightarrow Q_{i}$ таких, что $w_{i} p_{i}=q_{i} w_{i+1}$ для каждого $i$. Скажем, что $w$ удовлетворяет свойству поднятия гомотопии, если для каждого $i$ существует $j>i$ такое, что для любого наперед заданного компактного полиэдра $K$, гомотопии $f_{t}: K \rightarrow Q_{j}$ и поднятия $\bar{f}_{0}: K \rightarrow P_{j}$ отображения $\bar{f}_{0}$ (т. е. $w_{j} \bar{f}_{0}=f_{0}$ ) существует поднятие $\tilde{f}_{t}: K \rightarrow P_{i}$ отображения $q_{i}^{j} f_{t}$ (т. е. $w_{i} \tilde{f}_{t}=q_{i}^{j} f_{t}$ ) такое, что $\tilde{f}_{0}=p_{i}^{j} \bar{f}_{0}$. Наконец, отображение $f: E \rightarrow B$ между компактами назовем стинродовским расслоением, если $E=\lim _{\longleftarrow} P, B=\lim _{\longleftarrow} Q$ и $f=\lim _{\longleftarrow} w$ для некоторых $P, Q$ и $w$ как выше, причем $w$ удовлетворяет свойству поднятия гомотопии.

Ясно, что свойство поднятия гомотопии выполнено с $j(i)=i$, если

(i) каждое $f_{i}$ является расслоением в смысле Серра.

В частности, это так, если

(ii) каждое $f_{i+1}$ является композицией серровского расслоения $\varphi_{i+1}: P_{i+1} \rightarrow$ $R_{i+1}$ и пулбека (т. е. обратного образа) $q_{i}^{*}\left(f_{i}\right): R_{i+1} \rightarrow Q_{i+1}$ отображения $f_{i}$.

В последней ситуации (ii) $f$ будет и серровским расслоением, поскольку оно отождествлено с отображением $\psi_{0}^{\infty}$, соответствующим разложению $E=$ $\underset{\lim }{\longleftarrow}\left(\cdots \stackrel{\psi_{1}}{\longrightarrow} B_{1} \stackrel{\psi_{0}}{\longrightarrow} B_{0}=B\right)$, где каждое $\psi_{i}-$ пулбек отображения $\varphi_{i}$. В более общей ситуации (i) $f$ уже не обязано быть серровским расслоением, как показывают следующие примеры.

Кусочно линейное отображение $f:[0,2] \rightarrow[0,1], x \mapsto \min (x, 1)$, является стинродовским расслоением (даже с $f_{i}$, являющимися расслоениями), но не серровским расслоением. Другой такой пример - проекция синусоиды топологов (см. пример 2.10) на $[0,1]$. С другой стороны, если $B$ - компакт, не содержащий невырожденных путей (например, псевдодуга), то всякое отображение $f: E \rightarrow B$ является серровским расслоением. Как замечено в [72; пример 4], оно не обязано быть стинродовским расслоением, например, если $E=B \vee B$ (для произвольного выбора неизолированной базисной точки) и $f$ - складывающее отображение. 
Теорема 3.15. Пусть $f: E \rightarrow B$ - отображение между компактами

(а) Предположим, что $E=\lim _{\longleftarrow} R$ u $B=\lim _{\longleftarrow} Q$, где $R=\left(\cdots \stackrel{r_{1}}{\longrightarrow} R_{1} \stackrel{r_{0}}{\longrightarrow} R_{0}\right)$ u $Q=\left(\cdots \stackrel{q_{1}}{\longrightarrow} Q_{1} \stackrel{q_{0}}{\longrightarrow} Q_{0}\right)$ - обратные последовательности компактных полиэдров и кусочно линейных отображсений. Тогда существуют обратная последовательность $P=\left(\cdots \stackrel{p_{1}}{\longrightarrow} P_{1} \stackrel{p_{0}}{\longrightarrow} P_{0}\right)$ компактных полиэдров и кусочно линейных отображений $c \lim P=E$ u PL-поэтажсные отображения $u: P \rightarrow R$ $u w: P \rightarrow Q$ maкue, чmо $\overleftarrow{\lim } u=\operatorname{id}_{E} u \lim w=f$.

(b) $[73 ; 3.3]$ Предположим , что $f$-стинродовское расслоение, а точка $b \in B$ такова, что $F:=f^{-1}(b)$ непуст; выберем некоторую $\tilde{b} \in F:=f^{-1}(b)$. Тогда имеются естественные действия $\pi_{1}(E)$ на $\pi_{n}(F)$ при $n \geqslant 1$ и $\pi_{1}(B)$ на $\pi_{0}(F)$ и естественная точная последовательность пунктированных множеств

$$
\cdots \rightarrow \pi_{1}(F) \rightarrow \pi_{1}(E) \stackrel{f_{*}}{\longrightarrow} \pi_{1}(B) \rightarrow \pi_{0}(F) \rightarrow \pi_{0}(E) \stackrel{f_{*}}{\longrightarrow} \pi_{0}(B),
$$

отображения которой слева от $\pi_{1}(B)$ (соответственно $\left.\pi_{2}(B)\right)$ являются гомоморфизмами групп (соответственно правых $\mathbb{Z} \pi_{1}(E)$-модулей). Кроме того, $\pi_{2}(B) \rightarrow \pi_{1}(F)$ тоже $\pi_{1}(E)$-эквивариантен, $\pi_{1}(F) \rightarrow \pi_{1}(E)$ является скрещенным модулем, $\pi_{1}(B) \rightarrow \pi_{0}(F)$ является $\pi_{1}(B)$-эквивариантным относительно правого регулярного действия на $\pi_{1}(B)$, а нетривиальные прообразы точек при $\pi_{0}(F) \rightarrow \pi_{0}(E)$ совпадают с орбитами действия $\pi_{1}(B)$.

$\left(\mathrm{b}^{\prime}\right)$ В обозначениях пункта $(\mathrm{b})$, если $B$ связна, то $f_{*}: \pi_{0}(E) \rightarrow \pi_{0}(B)$ сюргективно.

(c) $[73 ; 3.4]$ Если $f$ - стинродовское расслоение и $b, b^{\prime} \in B$ представляют один и тот же элемент группы $\pi_{0}(B)$, то $f^{-1}(b)$ u $f^{-1}\left(b^{\prime}\right)$ имеют один и тот же шейn.

(d) Если E конечномерен $u f$ клеточноподобно, т.е. компакт $f^{-1}(b)$ клеточноподобен для каждой $b \in B$, то $f$ - стинродовское расслоение.

(е) [23] Клеточноподобное отображение между связными конечномерными компактами является стинродовской гомотопической эквивалентностью.

Из части (а) следует, что класс стинродовских расслоений совпадает с (априори более широким) классом шейповых расслоений, которые были введены в $[72]{ }^{17}$

Ослабленная версия части (c) была первоначально доказана в [72; теорема 3]. Часть (d) усиливает теорему 5 работы [72]. Ослабленные версии (d) были первоначально получены Дж. Дыдаком (1975) и К. Моритой (1975) (см. [67; теорема 8.5]), доработавшими более ранние результаты С. А. Богатого [74], Ю. Кодамы (1974) и К. Куперберг [75].

Предположение конечномерности необходимо в (е) (см. [29; пример 10.3.1]), а также в (d) [72; пример 6].

ДоказАтельство. (а) Пусть $\Gamma \subset E \times B-$ график отображения $f$. Пусть $P_{i}-$ полиэдральная окрестность образа графика $\Gamma$ в $R_{i} \times Q_{i}$ такая, что каждое $P_{i+1}$

\footnotetext{
${ }^{17}$ Покажем больше: если $f$ - шейповое расслоение, а $R, Q$ - такие, как в (а), то существуют $P, u$ и $w$ такие, как в (а), и такие, что $w$ удовлетворяет свойству поднятия гомотопии. В самом деле, используя (а), мы можем предполагать, что имеется PL-поэтажное отображение $v: R \rightarrow Q$ с $\lim v=f$. Тогда, по теореме 1 из [72], $v$ удовлетворяет свойству AHLP из [72]. Требуемые $P, u$ и $w$ теперь доставляются доказательством теоремы 2 из [72].
} 
отображается в $P_{i}$ и $\lim P_{i}=\Gamma$. Определим $u_{i}: P_{i} \rightarrow R_{i}$ и $w_{i}: P_{i} \rightarrow Q_{i}$ как ограничения проекций на сомножители произведения $R_{i} \times Q_{i}$.

(b) Пусть $P, Q$ и $w$ - такие, как в определении стинродовского расслоения, причем $j(i)=i+1$ в свойстве поднятия гомотопии.

Докажем свойство поднятия гомотопии для сохраняющих уровни представителей стинродовских гомотопических классов. Рассмотрим полиэдр $X$ и сохраняющее уровни отображение $\varphi_{[0, \infty)}: X \times I \times[0, \infty) \rightarrow Q_{[0, \infty)}$ вместе с поднятием $\bar{\varphi}_{[0, \infty]}: X \times[0, \infty) \rightarrow P_{[0, \infty)}$ его ограничения на множество $X \times\{0\} \times$ $[0, \infty)$. По условию каждый его сдвинутый уровень $q_{i-1} \varphi_{i}$ поднимается в отображение $\tilde{\varphi}_{i-1}: X \times I \rightarrow P_{i}$, сужающееся в $p_{i-1} \bar{\varphi}_{i}$ на $X \times\{0\}$. Поскольку $q_{[i-2, i-1]}^{[i, i+1]}: Q_{[i, i+1]} \rightarrow Q_{[i-2, i-1]}$ пропускается через $q_{i-1} \times \operatorname{id}_{I}: Q_{i} \times I \rightarrow Q_{i-1} \times I$, мы также получаем поднятие $\tilde{\varphi}_{[i-2, i-1]}$ композиции $q_{[i-2, i-1]}^{[i, i+1]} \varphi_{[i, i+1]}$, сужающееся до $p_{[i-2, i-1]}^{[i, i+1]} \bar{\varphi}_{[i, i+1]}$ на $X \times\{0\} \times[i, i+1]$. Пара последовательных применений этого построения доставляет два потенциально различных поднятия $\tilde{\varphi}_{i-2}^{\prime}$ и $\tilde{\varphi}_{i-2}^{\prime \prime}$ композиции $q_{i-2}^{i} \varphi_{i}$, сужающихся до $p_{i-2}^{i} \bar{\varphi}_{i}$ на $X \times\{0\}$. По условию $p_{i-3} \tilde{\varphi}_{i-2}^{\prime}$ гомотопно $p_{i-3} \tilde{\varphi}_{i-1}^{\prime \prime}$ в классе поднятий отображения $q_{i-3}^{i} \varphi_{i}$, сужающихся в $p_{i-3}^{i} \bar{\varphi}_{i}$ на $X \times\{0\}$. Отсюда следует, что $p_{[i-3, i-2]} \tilde{\varphi}_{[i-2, i-1]}$ гомотопно с носителем над $[i-3, i-2.5]$ новому поднятию $\tilde{\varphi}_{[i-3, i-2]}^{\prime}$ композиции $q_{[i-3, i-2]}^{[i, i+1]} \varphi_{[i, i+1]}$, теперь уже согласованному со своей соседкой $\tilde{\varphi}_{[i-4, i-3]}^{\prime} \cdot$ Таким образом, мы получаем поднятие $\tilde{\varphi}_{[0, \infty)}: X \times[0, \infty) \rightarrow P_{[0, \infty)}$ композиции $q_{[-3, \infty-3]}^{[0, \infty)} \varphi_{[0, \infty)}$, сужающееся до $p_{[-3, \infty-3]}^{[0, \infty)} \bar{\varphi}_{[0, \infty)}$ на $X \times\{0\} \times[0, \infty)$.

Из установленного свойства легко вытекает биективность индуцированного включением отображения $\pi_{n}(C F, F ; \tilde{b}) \rightarrow \pi_{n}(M C(f), E ; \tilde{b})$ при $n \geqslant 1 .^{18}$ Поскольку $C F$ клеточноподобен, будучи обратным пределом конусов, то отображение $\partial: \pi_{n}(C F, F ; \tilde{b}) \rightarrow \pi_{n-1}(F ; \tilde{b})$ биективно по теореме $3.1(\mathrm{a})$. Тогда утверждение следует из точной гомотопической последовательности пары $(M C(f), E)$, доставляемой теоремой $3.1(\mathrm{a})$.

$\left(\mathrm{b}^{\prime}\right)$ Можно считать, что $E$ связно; в противном случае можно заменить его связной компонентой, содержащей $\tilde{b}$, и проверить, что ограничение отображения $f$ является стинродовским расслоением. Пусть $P, Q$ и $w$ - такие, как в определении стинродовского расслоения, с $j(i)=i+1$ в свойстве поднятия гомотопии. Тогда по теореме $3.1(\mathrm{~b})$ отображение $\pi_{0}(E) \rightarrow \pi_{0}(B)$ можно отождествить с $\lim ^{1}\left(w_{i}\right)_{*}: \lim ^{1} \pi_{1}\left(P_{i}\right) \rightarrow \lim ^{1} \pi_{1}\left(Q_{i}\right)$. Без ограничения общности можно считать, что все $\check{P}_{i}$ связны, значит, коядро $\left(w_{i}\right)_{*}: \pi_{1}\left(P_{i}\right) \rightarrow \pi_{1}\left(Q_{i}\right)$ можно отождествить с $S_{i}:=\pi_{0}\left(M C\left(w_{i}\right), P_{i}\right)$. Ввиду леммы 3.7 (а) достаточно показать, что обратная последовательность $\cdots \rightarrow S_{1} \rightarrow S_{0}$ удовлетворяет условию Миттаг-Леффлера. В самом деле, пусть $F_{i}=w_{i}^{-1}\left(b_{i}\right)$, где $b_{i}=p_{i}^{\infty}(b)$. В силу свойства поднятия гомотопии каждое связующее отображение $S_{i+1} \rightarrow S_{i}$ пропускается через $T_{i}:=\pi_{1}\left(M C\left(\left.w_{i}\right|_{F_{i}}\right), F_{i}\right)$. Каждое $T_{i}$ можно отождествить с $\pi_{0}\left(F_{i}\right)$, и потому оно конечно. Значит, $S_{i}$ удовлетворяют условию МиттагЛеффлера.

\footnotetext{
18 Другой возможный подход основан на том, что $f_{*}: \pi_{n}(E, F ; \tilde{b}) \rightarrow \pi_{n}(B ; b)$ биективно. Это приводит к более слабым заключениям: точная последовательность становится короче на один член; $\pi_{1}(E)$-действия сводятся, посредством ограничения скаляров, к $\pi_{1}(F)$-действиям; $\pi_{1}(B)$-действие сводится к $\pi_{1}(E)$-действию.
} 
(c) Пусть $P, Q$ и $w$ - такие, как в определении стинродовского расслоения, причем $j(i)=i+1$ в свойстве поднятия гомотопии. Пусть $R_{i}=w_{i}^{-1}\left(b_{i}\right)$ и $R_{i}^{\prime}=w_{i}^{-1}\left(b_{i}^{\prime}\right)$, где $b_{i}=q_{i}^{\infty}(b)$ и $b_{i}^{\prime}=q_{i}^{\infty}\left(b^{\prime}\right)$, и пусть $v: R_{[0, \infty)} \rightarrow[0, \infty)-$ сохраняющая уровни проекция. Выберем сохраняющий уровни стинродовский путь $\ell_{t}:[0, \infty) \rightarrow Q_{[0, \infty)}$, соединяющий $b_{[0, \infty)}$ с $b_{[0, \infty)}^{\prime}$. Тогда по доказательству части $(\mathrm{b})$ собственная гомотопия $q_{(-3, \infty-3)}^{[0, \infty)} v \ell_{t}: R_{[0, \infty)} \rightarrow Q_{[0, \infty)}$ поднимается в гомотопию $h_{t}: R_{[0, \infty)} \rightarrow P_{[0, \infty)}$ такую, что $h_{0}$ есть композиция включения и $p_{(-3, \infty-3)}^{[0, \infty)}$. В частности, мы получаем собственное отображение $h_{1}: R_{[0, \infty)} \rightarrow R_{[0, \infty)}^{\prime}$; аналогично конструируем $h_{1}^{\prime}: R_{[0, \infty)}^{\prime} \rightarrow R_{[0, \infty)}$. Так как складывающее отображение $[-1,1] \rightarrow[0,1], x \mapsto|x|$, нульгомотопно неподвижно на концах, то $h_{1} h_{1}^{\prime}$ и $h_{1}^{\prime} h_{1}$ собственно гомотопны тождественному отображению.

(d) Пусть $P, Q$ и $w$ - такие, как в (а), причем $\operatorname{dim} P_{i} \leqslant m$ для каждого $i$. Триангулируем каждое $Q_{i}$ так, чтобы каждое $q_{i}$ было симплициальным относительно триангуляции $Q_{i+1}$ и некоторого измельчения второго барицентрического измельчения триангуляции $Q_{i}$. Выберем точку $v \in B$, и для каждого $i$ пусть $N_{i}^{v}$ - симплициальная окрестность $q_{i}^{\infty}(v)$ в $Q_{i}$. Тогда каждое $q_{i}\left(N_{i+1}^{v}\right)$ лежит в $N_{i}^{v}$ и $\lim N_{i}^{v}=\{v\}$. Более того, объединение всех $N_{i+1}^{u}$, пересекающих $N_{i+1}^{v}$, отображено в $N_{i}^{v}$ благодаря нашему выбору триангуляций. Пусть $M_{i}^{v}=w_{i}^{-1}\left(N_{i}^{v}\right)$. Используя клеточноподобность $f^{-1}(v)=\lim _{i} M_{i}^{v}$ и неравенство $\operatorname{dim} P_{i} \leqslant m$, по доказательству предложения 3.5 (а) мы можем предполагать, что каждое $\left.p_{i}\right|_{M_{i+1}^{v}}: M_{i+1}^{v} \rightarrow M_{i}^{v}$ нульгомотопно.

Пусть $Q_{i}^{\prime}=\bigcup_{v \in B} N_{i}^{v}$, другими словами, $Q_{i}^{\prime}$ - симплициальная окрестность $q_{i}^{\infty}(B)$, и пусть $P_{i}^{\prime}=\bigcup_{v \in B} N_{i}^{v} \times M_{i}^{v} \subset Q_{i}^{\prime} \times P_{i}$ (заметим, что объединение конечно). Пусть $p_{i}^{\prime}=\left.\left(q_{i} \times p_{i}\right)\right|_{P_{i}^{\prime}}$ и $q_{i}^{\prime}=\left.q_{i}\right|_{Q_{i}^{\prime}}$, и пусть $w_{i}^{\prime}: P_{i}^{\prime} \rightarrow Q_{i}^{\prime}$ - ограничение проекции. Для любого наперед заданного линейного отображения $\varphi: \Delta^{n+1} \rightarrow Q_{i+2}^{\prime}$ и частичного поднятия $\bar{\varphi}_{\partial}: \partial \Delta^{n+1} \rightarrow P_{i+2}^{\prime}$ имеем $\varphi\left(\Delta^{n+1}\right) \subset N_{i+2}^{v}$ для некоторого $v \in X$ и, следовательно, $\bar{\varphi}_{\partial}\left(\partial \Delta^{n+1}\right)$ лежит в объединении всех произведений $N_{i+2}^{u} \times M_{i+2}^{u}$, где $N_{i+2}^{u}$ пересекает $N_{i+2}^{v}$. Тогда $p_{i+1}^{\prime} \bar{\varphi}_{\partial}\left(\partial \Delta^{n+1}\right) \subset N_{i+1}^{v} \times M_{i+1}^{v}$. Пусть $\tilde{\varphi}_{\partial}: \partial \Delta^{n+1} \rightarrow M_{i+1}^{v}-$ композиция $p_{i+1}^{\prime} \bar{\varphi}_{\partial}$ и проекции. Тогда сдвиг $p_{i} \tilde{\varphi}_{\partial}: \partial \Delta^{n+1} \rightarrow M_{i}^{v}$ продолжается до отображения $\tilde{\varphi}: \Delta^{n+1} \rightarrow M_{i}^{v}$. Пусть $\bar{\varphi}:=\left(q_{i}^{i+2} \varphi\right) \times \tilde{\varphi}: \Delta^{n+1} \rightarrow N_{i}^{v} \times M_{i}^{v}$. Таким образом, $\bar{\varphi}: \Delta^{n+1} \rightarrow P_{i}^{\prime}-$ поднятие отображения $q_{i}^{\prime} q_{i+1}^{\prime} \varphi$, продолжающее $p_{i}^{\prime} p_{i+1}^{\prime} \bar{\varphi}_{\partial}$. В частности, $w^{\prime}: P^{\prime} \rightarrow Q^{\prime}$ удовлетворяет свойству поднятия гомотопии.

(e) Следует из частей $(\mathrm{b})$ и $(\mathrm{d})$, соединенных с теоремой 3.6.

\section{§ 4. Гомологии и когомологии}

Гомологии Стинрода. Следуя [34; $\mathrm{IV}]$, мы определяем $H_{n}(X, A)$ как группу локально-конечных гомологий $H_{n+1}^{\mathrm{lf}}\left(P_{[0, \infty)}, P_{0} \cup Q_{[0, \infty)}\right)$, где $(X, A)-$ предел обратной последовательности компактных полиэдральных пар $\left(P_{i}, Q_{i}\right)$. Напомним, что локально-конечные гомологии $H_{i}^{\mathrm{lf}}\left(P_{[0, \infty)}, P_{0} \cup Q_{[0, \infty)}\right)$ можно определить как группу классов ориентированных собственных псевдобордизмов собственных отображений из ориентированных $i$-псевдомногообразий $(M, \partial M)$ в $\left(P_{[0, \infty)}, P_{0} \cup Q_{[0, \infty)}\right)$. Так как локально-конечные гомологии являют- 
ся инвариантом собственного гомотопического типа, то $H_{n}(X, A)$ - шейповый инвариант пары $(X, A)$ ввиду следствия 2.2. (Отсюда, в частности, следует топологическая инвариантность гомологий Стинрода.) Индуцированные отображения $f_{*}$, где $f$ - отображение или стинродовский гомотопический класс, и граничные отображения $\delta_{*}$ (которые также являются стинродовскими гомотопическими инвариантами) определяются очевидным образом.

Теорема 4.1 (Милнор [13]). Гомологии Стинрода удовлетворяют всем аксиомам Эйленберга-Стинрода и, кроме того, обладают следующими свойствами:

(i) Аксиома вырезания для отображений: $f_{*}: H_{n}(X, A) \rightarrow H_{n}(Y, B)-$ изоморфизм для произвольного отображения $f:(X, A) \rightarrow(Y, B)$, сужающегося до гомеоморфизма $X \backslash A \rightarrow Y \backslash B$.

(ii) Аксиома грозди: $H_{n}\left(\left(\bigsqcup X_{i} \backslash \mathrm{pt}\right)^{+}\right.$, pt $) \simeq \prod H_{n}\left(X_{i}\right)$ естественно для произвольных компактов $X_{1}, X_{2}, \ldots$, где $Y^{+}$обозначает одноточечную компактификаиию $Y$.

(iii) Точная последовательность Милнора: если $(X, A)$ есть обратный предел $\underset{\lim }{\longleftarrow}\left(\cdots \stackrel{q^{1}}{\longrightarrow}\left(Y_{1}, Z_{1}\right) \stackrel{q^{0}}{\longrightarrow}\left(X_{0}, Z_{0}\right)\right)$, где $\left(Y_{i}, Z_{i}\right)$ - пары компактов, то существует естественная короткая точная последовательность групп

$$
0 \rightarrow \lim ^{1} H_{n+1}\left(Y_{i}, Z_{i}\right) \rightarrow H_{n}(X, A) \rightarrow \lim _{\longleftarrow} H_{n}\left(Y_{i}, Z_{i}\right) \rightarrow 0 .
$$

(iv) Стинродовские гомологии - единственная ординарная теория гомологий (в смысле аксиом Эйленберга-Стинрода) на парах компактов, удовлетворяющая аксиоме (ii) для компактных полиэдров $X_{i}$ и аксиоме (i).

Отметим, что (ii) есть частный случай (iii), что легко видеть, если учесть, что гроздъ $\left(\bigsqcup X_{i} \backslash \mathrm{pt}\right)^{+}$гомеоморфна $\lim \left(\cdots \rightarrow X_{1} \vee X_{2} \vee X_{3} \rightarrow X_{1} \vee X_{2} \rightarrow X_{1}\right)$.

Некоторая форма утверждения (iii) фигурирует уже в [34; теорема 8].

Понтрягинские когомологии. Группа $H^{n}(X, A)$ определяется как группа когомологий с компактным носителем $H_{\mathrm{c}}^{n+1}\left(P_{[0, \infty)}, P_{0} \cup Q_{[0, \infty)}\right)$, где $(X, A)-$ предел обратной последовательности компактных полиэдральных пар $\left(P_{i}, Q_{i}\right)$. Замечания, аналогичные сделанным об $H_{n}(X, A)$, применимы и к когомологиям, и $H_{n}(X, A ; G)$ может быть определена аналогичным образом. Кроме того,

$$
H_{\mathrm{c}}^{n+1}\left(P_{[0, \infty)}, P_{0}\right) \simeq \lim _{\longrightarrow} H^{n+1}\left(P_{[0, \infty)}, P_{0} \cup P_{[k, \infty)}\right) \simeq \underline{\lim _{\longrightarrow}} H^{n}\left(P_{[k, \infty)}\right) \simeq \underline{\lim } H^{n}\left(P_{k}\right),
$$

где второй изоморфизм имеет место, поскольку $H^{n+1}\left(P_{[0, \infty)}, P_{0}\right)=0$ ввиду того, что $P_{[0, \infty)}$ деформационно ретрагируется на $P_{0}$, а третий - поскольку $P_{[k, \infty)}$ деформационно ретрагируется на $P_{k}$. Сквозной изоморфизм

$$
H^{n}(X) \simeq \underset{\lim }{\longrightarrow} H^{n}\left(P_{k}\right)
$$

представляет собой первоначальное определение группы $H^{n}(X)$, принадлежащее в конечномерном случае Понтрягину [35; гл. III, § II]. В качестве следствия этого изоморфизма и предложения 2.3 (b) получаем следующее утверждение.

ПреДЛОЖЕНИЕ 4.2. Существует естественный изоморфизм $H^{n}(X ; G) \simeq$ $[X, K(G, n)]$. 
Когомологический аналог теоремы 4.1 имеет место, с заменой прямого произведения в (ii) прямой суммой, а точной последовательности (iii) - естественным изоморфизмом $H^{n}(X, A) \simeq \underset{\lim }{\longrightarrow} H^{n}\left(Y_{i}, Z_{i}\right)$ [13].

ТеОрема 4.3. Если $X \subset \mathbb{R}^{m}$ - компакт, то существуют естественные (относительно включений) изоморфизмы $H^{n}(X) \simeq \widetilde{H}_{m-n-1}\left(\mathbb{R}^{m} \backslash X\right)$ u $H_{n}(X) \simeq$ $\widetilde{H}^{m-n-1}\left(\mathbb{R}^{m} \backslash X\right)$.

Второй изоморфизм был установлен Стинродом [34]. Первый изоморфизм восходит к первоначальной теореме двойственности Александера (1922), которая была сформулирована для чисел Бетти по модулю 2 и для полиэдров $X$ (быть может, дико вложенных). Эти ограничения были постепенно сняты Александровым, Франклем, Лефшецем и в конечном счете Понтрягиным (см. работу [35] и ссылки там).

Как известно, при принятии аксиомы вырезания для отображений эти изоморфизмы становятся очевидными, если не принимать в расчет использование двойственности Пуанкаре на некомпактном многообразии $S^{m} \backslash X$ (см., например, [76]). Поскольку проверка аксиомы вырезания для отображений - предмет для отдельного обсуждения, полезно иметь в виду и непосредственную версию этого рассуждения:

ДокАЗАТЕЛЬство. Пусть $\cdots \subset U_{2} \subset U_{1} \subset U_{0}$ - последовательность полиэдральных окрестностей $X$ такая, что $\bigcap U_{i}=X$. Тогда из определения $H_{n}(X)$ и подходящего варианта двойственности Пуанкаре получаем

$$
H_{n}(X) \simeq H_{n+1}^{\mathrm{lf}}\left(U_{[0, \infty)}, U_{0}\right) \simeq H^{m-n}\left(U_{[0, \infty)}, \operatorname{Fr} U_{[0, \infty)}\right)
$$

Используя вырезание, точную последовательность пары и тривиальность группы $\widetilde{H}^{i}\left(\mathbb{R}^{m} \times[0, \infty)\right)$, заключаем, что последняя группа изоморфна

$$
\widetilde{H}^{m-n-1}\left(\mathbb{R}^{m} \times[0, \infty) \backslash U_{[0, \infty)}\right) \simeq \widetilde{H}^{m-n-1}\left(\mathbb{R}^{m} \backslash X\right) .
$$

Последний изоморфизм имеет место, поскольку проекция $\mathbb{R}^{m} \times[0, \infty) \backslash U_{[0, \infty)} \rightarrow$ $\mathbb{R}^{m} \backslash X$ является гомотопической эквивалентностью. Аналогично,

$$
\begin{aligned}
H^{n}(X) & \simeq H_{\mathrm{c}}^{n+1}\left(U_{[0, \infty)}, U_{0}\right) \simeq H_{m-n}\left(U_{[0, \infty)}, \operatorname{Fr} U_{[0, \infty)}\right) \\
& \simeq \widetilde{H}_{m-n-1}\left(\mathbb{R}^{m} \times[0, \infty) \backslash U_{[0, \infty)}\right) \simeq \widetilde{H}_{m-n-1}\left(\mathbb{R}^{m} \backslash X\right)
\end{aligned}
$$

ЗАмечАниЕ. Приведенное рассуждение доставляет элементарное доказательство известной теоремы Жордана, использующее только простейшие факты о (ко)гомологиях полиэдра, двойственность Пуанкаре и лемму 2.1 (последняя необходима по той причине, что $H^{1}\left(S^{1}\right)=H_{\mathrm{c}}^{2}\left(P_{[0, \infty)}, P_{0}\right)$ вычисляется дважды: при $P_{i}=U_{i}$ и при $\left.P_{i}=S^{1}\right)$.

По аналогии с интерпретацией понтрягинских когомологий как прямого предела удобно зарезервировать специальное обозначение для обратных пределов, фигурирующих в коротких точных последовательностях Милнора. 
Чеховские гомотопии и гомологии. Если $X$ - обратный предел полиэдров $P_{i}$, то рассмотрим топологическую группу (пунктированное пространство при $n=0) \check{\pi}_{n}(X, x):=\lim _{n}\left(P_{i}, p_{i}\right)$ и еще топологическую группу $\check{H}_{n}(X):=$ $\lim H_{n}\left(P_{i}\right)$. По предложению $2.6 \check{\pi}_{n}$ (соответственно $\left.\check{H}_{n}\right)$, да и вся короткая точная последовательность теоремы 3.1 (b) (соответственно теоремы 4.1 (iii)) является шейповым инвариантом $(X, x)$ (соответственно $X)$. Канонические эпиморфизмы $\pi_{n}(X) \rightarrow \check{\pi}_{n}(X)$ и $H_{n}(X) \rightarrow \check{H}_{n}(X)$ будем обозначать $\check{\tau}$.

Вот (абсолютная) “теорема Гуревича для стинродовских гомотопий".

Tеорема 4.4. Пусть $m>1$ u $X$ - компакт такой, что $\pi_{n}(X)=0$ для всех $n<m$. Тогда гомоморфизм Гуревича $\pi_{n}(X) \rightarrow H_{n}(X)$ является изоморфизмом при $n \leqslant m$ и эпиморфизмом при $n=m+1$.

Первое утверждение теоремы 4.4 было высказано в качестве гипотезы в [32; c. 300] и доказано 40 лет спустя Лисицей [61]. Его доказательство второго утверждения, однако, содержит ошибку: в случае $m=2$ оно опирается на предположение, что если $X$ - предел обратной последовательности полиэдров $P_{i}$, то уже $\lim ^{1} \pi_{m+2}\left(P_{i}\right) \rightarrow \lim ^{1} H_{m+2}\left(P_{i}\right)$ якобы является эпиморфизмом. Однако

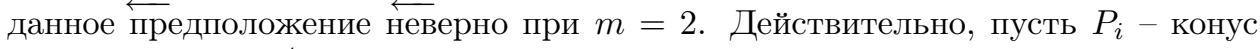
композиции $S^{3} \stackrel{p^{i}}{\longrightarrow} S^{3} \stackrel{h}{\longrightarrow} S^{2}$ отображения степени $p^{i}$ и отображения Хопфа. Тождественное отображение $S^{2}$ продолжается до отображения $f_{i}: P_{i+1} \rightarrow P_{i}$; пусть $X=\lim _{\longleftarrow}\left(\cdots \rightarrow P_{2} \rightarrow P_{1}\right)$. Очевидно, что каждое $\left(f_{i}\right)_{*}: H_{4}\left(P_{i+1}\right) \rightarrow H_{4}\left(P_{i}\right)$ является включением $\mathbb{Z} \stackrel{p}{\rightarrow} \mathbb{Z}$ на подгруппу индекса $p$, поэтому $\lim ^{1} H_{4}\left(P_{i}\right) \neq 0$. Так как $P_{i}$ односвязны, то каждое отображение $S^{4} \rightarrow P_{i}$ гомотопно такому, при котором вершина конуса имеет ровно один прообраз; отсюда вытекает, что $\pi_{4}\left(h p^{i}\right)$ отображается сюръективно на $\pi_{4}\left(P_{i}\right)$. Из точной последовательности

$$
\pi_{4}\left(S^{2}\right) \rightarrow \pi_{4}\left(h p^{i}\right) \rightarrow \pi_{3}\left(S^{3}\right) \stackrel{h p^{i}}{\longrightarrow} \pi_{3}\left(S^{2}\right)
$$

заключаем, что $\pi_{4}\left(h p^{i}\right)$ является факторгруппой группы $\pi_{4}\left(S^{2}\right) \simeq \mathbb{Z} / 2$. Значит, каждая $\pi_{4}\left(P_{i}\right)$ конечна и $\lim ^{1} \pi_{4}\left(P_{i}\right)=0$. Поэтому $\lim ^{1} \pi_{4}\left(P_{i}\right) \rightarrow \underset{\lim ^{1}}{\longleftarrow} H_{4}\left(P_{i}\right)$ не может быть сюръективным.

ЗАмЕЧАНИЕ. В приведенном примере положение можно поправить, используя сюръективность связующего гомоморфизма $\lim \pi_{3}\left(P_{i}\right) \rightarrow \lim ^{1} H_{4}\left(P_{i}\right)$ для короткой точной последовательности обратных последовательностей

$$
0 \rightarrow H_{4}\left(P_{i}\right) \rightarrow \Gamma_{3}\left(P_{i}\right) \rightarrow \pi_{3}\left(P_{i}\right) \rightarrow 0,
$$

полученной из леммы 4.5 ниже. Действительно, каждое $\Gamma_{3}\left(P_{i+1}\right) \rightarrow \Gamma_{3}\left(P_{i}\right)$ есть изоморфизм (между экземплярами $\mathbb{Z})$. Значит, $\lim ^{1} \Gamma_{3}\left(P_{i}\right)=0$ и, стало быть, связующий гомоморфизм должен быть сюръективен.

ЛЕмма 4.5 (Уайтхед [77]). Пусть $K$ - односвязный полиэдр с фиксированной триангуляиией, а $\Gamma_{n}(K)$ - образ отображения $\pi_{n}\left(K^{(n-1)}\right) \rightarrow \pi_{n}\left(K^{(n)}\right)$. Гомоморфизмы Гуревича включаются в длинную точную последовательность

$$
\cdots \rightarrow \Gamma_{n}(K) \rightarrow \pi_{n}(K) \rightarrow H_{n}(K) \rightarrow \Gamma_{n-1}(K) \rightarrow \cdots
$$


Использование геометрического языка позволяет упростить рассуждение Уайтхеда.

ДокАзАтельство. Утверждение леммы становится очевидным, если $\Gamma_{n}$ заменить группой $\Gamma_{n}^{\prime}$ классов $\partial$-сферических сингулярных ориентированных псевдобордизмов $\partial$-сферических сингулярных ориентированных $(n+1)$-псевдомногообразий в $K$. (Говорим, что $n$-псевдомногообразие $M$ с краем $\partial$-сберическое, если $\partial M$ PL-гомеоморфно $S^{n-1}$. Псевдобордизм $W$ между такими $M_{0}, M_{1} \partial$-сферический, если замыкание $\partial W \backslash\left(\partial M_{0} \cup \partial M_{1}\right)$ PL-гомеоморфно $S^{n-1} \times I$.) Поскольку $K$ односвязно, то $\Gamma_{n}^{\prime}$ изоморфна группе $\Gamma_{n}^{\prime \prime}$ классов односвязных $\partial$-сферических сингулярных ориентированных псевдобордизмов односвязных $\partial$-сферических сингулярных ориентированных $(n+1)$-псевдомногообразий в $K$.

Если $M-\partial$-сферическое ориентированное $(n+1)$-псевдомногообразие, то оно сдавливается на объединение $L$ остова $M^{(n-1)}$ с некоторыми $n$-симплексами $M$. Если, кроме того, $M$ односвязно, то гомоморфизм Гуревича $\pi_{n}\left(L, L^{(n-1)}\right) \rightarrow$ $H_{n}\left(L, L^{(n-1)}\right)$ является изоморфизмом. Далее, $[\partial M] \in H_{n}(M)$ тривиален, в частности, он отображается тривиально в $H_{n}\left(L, L^{(n-1)}\right)$. Отсюда вытекает, что включение $\partial M \subset M$ гомотопно отображению $\partial M \rightarrow L^{(n-1)}=M^{(n-1)}$. Аналогично, если $W$ - односвязный $\partial$-сферический ориентированный псевдобордизм между $M_{0}$ и $M_{1}$, то любые отображения $\partial M_{0} \rightarrow M_{0}^{(n-1)}$ и $\partial M_{1} \rightarrow M_{1}^{(n-1)}$, гомотопные включениям $\partial M_{0} \subset M_{0}$ и $\partial M_{1} \subset M_{1}$, гомотопны со значениями B $W^{(n)}$.

ДОКАЗАТЕЛЬСТВо ТЕОРЕМЫ 4.4. По предложению 3.5 (а) $X$ - предел обратной последовательности $(m-1)$-связных полиэдров $P_{i}$. Очевидно, что гомоморфизмы Гуревича включаются в длинную точную последовательность

$$
\cdots \rightarrow \Gamma_{n}^{\prime}(X) \rightarrow \pi_{n}(X) \rightarrow H_{n}(X) \rightarrow \Gamma_{n-1}^{\prime}(X) \rightarrow \cdots,
$$

где $\Gamma_{n}^{\prime}(X)$ - группа классов собственных сингулярных ориентированных псевдобордизмов, сужающихся до собственной гомотопии на крае, собственных сингулярных ориентированных $(n+2)$-псевдомногообразий с краем $S^{n} \times[0, \infty)$ в $P_{[0, \infty)}$. Аналогично доказательству теоремы $3.1(\mathrm{~b})$, имеется короткая точная последовательность

$$
0 \rightarrow \lim ^{1} \Gamma_{n+1}^{\prime}\left(P_{i}\right) \rightarrow \Gamma_{n}^{\prime}(X) \rightarrow \lim _{\longleftarrow} \Gamma_{n}^{\prime}\left(P_{i}\right) \rightarrow 0
$$

где $\Gamma_{n}^{\prime}\left(P_{i}\right)$ определена в доказательстве леммы 4.5 и совпадает с $\Gamma_{n}\left(P_{i}\right)$, как показано в том доказательстве. Так как каждый $P_{i}$ является $(m-1)$-связным, то $\Gamma_{n}\left(P_{i}\right)=0$ при $n \leqslant m$. Из условия и теоремы $3.1(\mathrm{~b})$ следует, что $\lim ^{1} \pi_{m}\left(P_{i}\right)=0$, значит, по лемме $3.3 \pi_{m}\left(P_{i}\right)$ удовлетворяет условию Миттаг-Леффлера. Тогда по лемме 4.6 ниже $\Gamma_{m+1}\left(P_{i}\right)$ удовлетворяет условию Миттаг-Леффлера и, следовательно, $\lim ^{1} \Gamma_{m+1}\left(P_{i}\right)=0$. Таким образом, $\Gamma_{n}^{\prime}(X)=0$ при $n \leqslant m$, что приводит к искомому утверждению.

Лемма 4.6. Пусть $P_{i}$ - обратная последовательность $(m-1)$-связных полиэдров, где $m>1$. Если $\pi_{m}\left(P_{i}\right)$ удовлетворяют условию Миттаг-Лефблера, то ему удовлетворяют и $\Gamma_{m+1}\left(P_{i}\right)$. 
ДокАзАтЕЛЬство. Пусть $\pi_{m}\left(P_{k}\right)$ отображается на образ $\pi_{m}\left(P_{j}\right)$ в $\pi_{m}\left(P_{i}\right)$, $k>j$. Применяя клеточную аппроксимацию, имеем, что $\pi_{m}\left(P_{k}^{(m)}\right)$ отображается на образ $\pi_{m}\left(P_{j}^{(m)}\right)$ в $\pi_{m}\left(P_{i}^{(m+1)}\right)$. Возьмем какую-нибудь гомотопическую эквивалентность $f: \bigvee S^{m} \rightarrow P_{j}^{(m)}$, и пусть $\alpha_{1}, \ldots, \alpha_{r} \in \pi_{m}\left(\bigvee S^{m}\right)-$ базис, определяемый лепестками букета. Тогда существуют $\beta_{1}, \ldots, \beta_{r} \in \pi_{m}\left(P_{k}^{(m)}\right)$, отображающиеся на образы $\alpha_{1}, \ldots, \alpha_{r}$ в $\pi_{m}\left(P_{i}^{(m+1)}\right)$. Комбинируя их представителей, получаем отображение $g: \bigvee S^{m} \rightarrow P_{k}^{(m)}$ такое, что две композиции $h_{1}: \bigvee S^{m} \stackrel{g}{\rightarrow} P_{k}^{(m)} \rightarrow P_{i}^{(m+1)}$ и $h_{2}: \bigvee S^{m} \stackrel{f}{\rightarrow} P_{j}^{(m)} \rightarrow P_{i}^{(m+1)}$ индуцируют одно и то же отображение на $\pi_{m}$. Тогда $h_{1}$ и $h_{2}$ гомотопны. Так как $f$ обратимо с точностью до гомотопии, то $P_{j}^{(m)} \rightarrow P_{i}^{(m+1)}$ пропускается с точностью до гомотопии через $P_{k}^{(m)} \rightarrow P_{i}^{(m+1)}$. Следовательно, $\pi_{m+1}\left(P_{k}^{(m)}\right)$ отображается на образ $\pi_{m+1}\left(P_{j}^{(m)}\right)$ в $\pi_{m+1}\left(P_{i}^{(m+1)}\right)$. Значит, $\Gamma_{m+1}\left(P_{k}\right)$ отображается на образ $\Gamma_{m+1}\left(P_{j}\right)$ в $\Gamma_{m+1}\left(P_{i}\right)$.

ЗАмЕчАниЕ. В литературе присутствуют следующие варианты теоремы 4.4.

Д. Кристи $[32$; теорема $(5.12)]$ доказал, что если $X$ является $U V_{m-1}$-компактом, где $m>1$, то гомоморфизм Гуревича $\check{\pi}_{m}(X) \rightarrow \check{H}_{m}(X)$ является изоморфизмом. Этот же факт был переоткрыт К. Куперберг (1972) и частично Т. Портером (1973). М. Артин и Б. Мазур [59; 4.5] доказывают про-групповую версию этого результата, из которой дополнительно следует, что при тех же предположениях $\pi_{m-1}(X) \rightarrow H_{m-1}(X)$ тоже является изоморфизмом; более простое доказательство имеется в [29]. Оба этих результата вытекают напрямую из предложения 3.5 (а) и классической теоремы Гуревича.

Ю. Кодама и А. Кояма [78], [79] доказывают, что если компакт $X$ удовлетворяет условию $\mathrm{UV}_{m-1}$, где $m>2$, то гомоморфизм Гуревича $\pi_{m}(X) \rightarrow H_{m}(X)$ является изоморфизмом. Этот результат вытекает из доказательства теоремы 4.4, если взамен леммы 4.6 использовать, что каждая $\Gamma_{m+1}\left(P_{i}\right)$ конечна при $m>2$. (Последнее имеет место, поскольку $\pi_{m+1}\left(\bigvee S^{m}\right)$ конечна при $m>2$, что, в свою очередь, легко увидеть, используя конструкцию Понтрягина-Тома.) Как отмечено в [78], этот результат не распространяется на случай $m=2$, что видно из рассмотрения надстройки над $p$-адическим соленоидом для некоторого $p$.

Наконец, как отмечено в [80], из $\check{\pi}_{n}(X)=0$ для всех $n<3$ не следует, что $\check{\pi}_{3}(X) \simeq \check{H}_{3}(X)$. Действительно, рассмотрим двукратную надстройку над $p$-адическим соленоидом с нечетным $p$. Требуемое утверждение вытекает из того, что отображение из $S^{3}$ в себя нечетной степени индуцирует тождество на $\pi_{4}\left(S^{3}\right) \simeq \mathbb{Z} / 2$, что, в свою очередь, легко увидеть, используя конструкцию Понтрягина-Тома.

ПРЕДЛОЖЕНИЕ 4.7. Если $\pi_{0}(X)=0$, то гомоморфизм Гуревича $\check{\pi}_{1}(X) \rightarrow$ $\check{H}_{1}(X)$ сюрвективен - с ядром, равным замыканию коммутанта.

Первое утверждение было доказано Мардешичем и Унгаром, а сформулировано Дыдаком [81]. Оно не обобщается на случай связного компакта $X$ [81]. Если $E$ - гавайская серьга (см. пример 5.6), то коммутант группы $\check{\pi}_{1}(E)$ не замкнут в этой группе [81]. Ошибочная версия предложения 4.7, не учитывавшая эти два контрпримера к ней, была заявлена без доказательства в [32]. 
ДоКАЗАтельСтво. Предположим, что $X$ - обратный предел полиэдров $P_{i}$. Обозначим $\pi_{1}\left(P_{i}\right)=G_{i}$. Каждый $G_{i} \rightarrow H_{1}\left(P_{i}\right)$ сюръективен с ядром $G_{i}^{\prime}$. По теореме 3.1 (b) и лемме $3.3 G_{i}$ удовлетворяет условию Миттаг-Леффлера. Тогда коммутант устойчивого образа групп $G_{j}, j>i$, в $G_{i}$ является устойчивым образом их коммутантов $G_{j}^{\prime}, j>i$, поэтому $G_{i}^{\prime}$ также удовлетворяют условию Миттаг-Леффлера. Значит, $f: \lim G_{i} \rightarrow \lim H_{1}\left(P_{i}\right)$ - сюръекция. Так как $f-$ непрерывный гомоморфизм в абелеву группу, то его ядро содержит замыкание коммутанта группы $\lim G_{i}$. Обратно, если нить $\left(g_{0}, g_{1}, \ldots\right)$ принадлежит $\operatorname{ker} f$, то каждый $g_{i}$ принадлежит $G_{i}^{\prime}$, причем $g_{i}$ лежит в устойчивом образе групп $G_{j}$, $j>i$. Тогда $g_{i}=\left[h_{i 1}, h_{i 1^{\prime}}\right] \cdots\left[h_{i r}, h_{i r^{\prime}}\right]$, где каждый $h_{i k}$ лежит в устойчивом образе групп $G_{j}$. Пусть $\hat{g}_{i}=\left[h_{1}, h_{1}^{\prime}\right] \cdots\left[h_{r}, h_{r}^{\prime}\right]$ для некоторых прообразов $h_{k}$ элементов $h_{i k}$ в $\lim G_{j}$, тогда $\hat{g}_{0}, \hat{g}_{1}, \ldots$ сходится к нити $\left(g_{0}, g_{1}, \ldots\right)$. Предложение 4.7 доказано.

В заключение отметим, что если $X-$ связный компакт, то $\pi_{0}(X) \rightarrow H_{0}(X)$ является эпиморфизмом по классической теореме Гуревича и теореме 3.1 (b).

\section{§ 5. Некоторые примеры}

Этот параграф имеет двоякую функцию: проиллюстрировать рассмотрения предшествующих параграфов и мотивировать рассмотрения последующих.

Пример 5.1 (нуль-последовательность). Пусть $\mathbb{N}^{+}$- одноточечная компактификация счетного дискретного пространства $\mathbb{N}$. Из теоремы 4.1 (iі) следует, что $H_{0}\left(\mathbb{N}^{+}\right) \simeq \lim H_{0}(\{1, \ldots, i\}) \simeq \prod \mathbb{Z}$ (счетное произведение). С другой стороны, поскольку сингулярный 0-цикл имеет носитель лишь в конечном множестве точек, то $\stackrel{\Delta}{\tau}: \stackrel{\Delta}{H}_{0}\left(\mathbb{N}^{+}\right) \rightarrow H_{0}\left(\mathbb{N}^{+}\right)$- вложение на подгруппу $\langle(1,1, \ldots)\rangle \oplus(\bigoplus \mathbb{Z})$ (счетная сумма). В то же время, легко видеть, что как $\pi_{0}\left(\mathbb{N}^{+}\right)$, так и $\stackrel{\Delta}{\pi}_{0}\left(\mathbb{N}^{+}\right)$ гомеоморфны $\mathbb{N}^{+}$.

Пример 5.2 (канторово множество). Пусть $\mathbb{Z}_{p}=\underset{\lim }{\lim }\left(\cdots \rightarrow \mathbb{Z} / p^{2} \rightarrow \mathbb{Z} / p\right)-$ топологическая группа целых $p$-адических чисел, где каждая $\mathbb{Z} / p^{n}$ снабжена дискретной топологией. Имеем $\pi_{0}\left(\mathbb{Z}_{p}\right) \cong \lim _{0} \pi_{0}\left(\mathbb{Z} / p^{i}\right) \cong \mathbb{Z}_{p}$ (как пространства). Очевидно, что $\stackrel{\Delta}{0}_{0}\left(\mathbb{Z}_{p}\right) \stackrel{\stackrel{\Delta}{\tau}}{\rightarrow} \pi_{0}\left(\mathbb{Z}_{p}\right)$ - гомеоморфизм. С другой стороны, легко видеть, что $\stackrel{\Delta}{H}_{0}\left(\mathbb{Z}_{p}\right)$ изоморфна как топологическая группа аддитивной группе группового кольца $\mathbb{Z}\left[\mathbb{Z}_{p}\right]$. В то же время, уже единичный элемент группы $H_{0}\left(\mathbb{Z}_{p}\right) \simeq \lim H_{0}\left(\mathbb{Z} / p^{i}\right) \simeq \lim \mathbb{Z}\left[\mathbb{Z} / p^{i}\right]$ вполне может "вовлекать" все точки $\mathbb{Z}_{p}$. Например, элементы $\sum_{i=0}^{n-1} m_{i} t^{i} \in \mathbb{Z}\left[\mathbb{Z} / 2^{n}\right]$, где $m_{\alpha_{0}+2 \alpha_{1}+\cdots+2^{n-1} \alpha_{n-1}}=$ $(-1)^{\alpha_{0}}+(-1)^{\alpha_{1}} \cdot 2+\cdots+(-1)^{\alpha_{n}} \cdot 2^{n-1} \neq 0$ (здесь каждое $\alpha_{i}$ равно 0 или 1 ), образуют нить в $\lim _{\longleftarrow} \mathbb{Z}\left[\mathbb{Z} / 2^{n}\right] \simeq H_{0}\left(\mathbb{Z}_{2}\right)$.

ПримеР 5.3 ( тор гомеоморфизма $h: \mathbb{Z}_{p} \rightarrow \mathbb{Z}_{p}, h(a)=a+1$. Из точной последовательности пары $\left(\Sigma_{p}, \mathbb{Z}_{p}\right)$ получаем $0 \rightarrow \mathbb{Z} \stackrel{\partial}{\rightarrow} \mathbb{Z}_{p} \rightarrow \stackrel{\Delta}{\pi}_{0}\left(\Sigma_{p}\right) \rightarrow 0$ откуда $\stackrel{\Delta}{\pi}_{0}\left(\Sigma_{p}\right) \cong \mathbb{Z}_{p} / \mathbb{Z}$ (как пространства). Поскольку то же рассуждение применимо и к $\pi_{0}\left(\Sigma_{p}\right)$ (см. теорему $3.1(\mathrm{a}))$, то $\stackrel{\Delta}{\pi}_{0}\left(\Sigma_{p}\right) \stackrel{\stackrel{\Delta}{\tau}}{\rightarrow} \pi_{0}\left(\Sigma_{p}\right)-$ биекция. Стинродовская группа $\pi_{0}\left(\Sigma_{p}\right)$ 
может быть вычислена и иначе - с использованием точной последовательности Милнора из теоремы $3.1(\mathrm{~b}): \lim \pi_{0}\left(S^{1}\right)=$ pt, значит, $\pi_{0}\left(\Sigma_{p}\right) \simeq \lim ^{1} \pi_{1}\left(S^{1}\right) \simeq$ $\mathbb{Z}_{p} / \mathbb{Z}$ (см. пример 3.2).

Что же касается гомологий, $\stackrel{\Delta}{H}_{0}\left(\Sigma_{p}\right) \stackrel{\stackrel{\Delta}{\rightarrow}}{\rightarrow} H_{0}\left(\Sigma_{p}\right)$ имеет нетривиальное ядро, вопреки утверждению из [76; с. 152]. Имеем $\widetilde{H}_{0}\left(\Sigma_{p}\right)=\lim ^{1}(\cdots \stackrel{p}{\rightarrow} \mathbb{Z} \stackrel{p}{\rightarrow} \mathbb{Z}) \simeq$

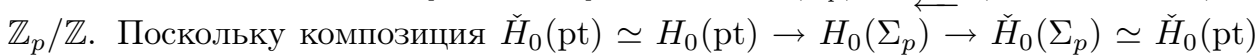
есть тождество, то $\check{H}_{0}\left(\Sigma_{p}\right)$ выделяется прямым слагаемым в $H_{0}\left(\Sigma_{p}\right)$. Значит, $H_{0}\left(\Sigma_{p}\right) \simeq \mathbb{Z} \oplus\left(\mathbb{Z}_{p} / \mathbb{Z}\right)$. С другой стороны, из точной последовательности пары $\left(\Sigma_{p}, \mathbb{Z}_{p}\right)$ получаем $\stackrel{\Delta}{H}_{0}\left(\Sigma_{p}\right) \simeq \mathbb{Z}\left[\mathbb{Z}_{p} / \mathbb{Z}\right]$. (Вычисление $H_{0}\left(\Sigma_{p}\right)$ из пары $\left(\Sigma_{p}, \mathbb{Z}_{p}\right)$ также возможно, но гораздо менее тривиально; см. [82; приложение].)

ЗАМЕчАНИЕ. В действительности, хорошо известно, что если $X$ - предел обратной последовательности расслоений в смысле Серра (или даже стинродовских расслоений) между полиэдрами, то $\stackrel{\Delta}{\pi}_{n}(X) \stackrel{\stackrel{\Delta}{\tau}}{\rightarrow} \pi_{n}(X)-$ биекция для всех $n$ [83], [53; гл. IX, теорема 3.1], [21; следствие 5.5(i)].

ПримеР 5.4 (гроздь соленоидов). Пусть $X$ - гроздь соленоидов, т. е. одноточечная компактификация счетного дизъюнктного семейства экземпляров проколотого соленоида $\Sigma_{p} \backslash$ pt с компактифицирующей точкой $b$. Мы утверждаем, что $\stackrel{\Delta}{\tau}: \stackrel{\Delta}{H}_{0}(X) \rightarrow H_{0}(X)$ не сюръективно. По аксиоме грозди (см. теорему 4.1), $H_{0}(X, b)$ естественно изоморфно счетному произведению экземпляров $\widetilde{H}_{0}\left(\Sigma_{p}\right) \simeq \mathbb{Z}_{p} / \mathbb{Z}$. Всякий сингулярный 0-цикл имеет носитель в некотором конечном подбукете $\Sigma_{p} \vee \cdots \vee \Sigma_{p}$ грозди. Значит, $\stackrel{\Delta}{H}_{0}(X, b)$ отображается в (в действительности, на) счетную сумму $\bigoplus \mathbb{Z}_{p} / \mathbb{Z} \subset \prod \mathbb{Z}_{p} / \mathbb{Z}$.

Пример 5.5 (букет соленоидов). Утверждается, что $\stackrel{\Delta}{\tau}: \stackrel{\Delta}{\pi}_{0}(X) \rightarrow \pi_{0}(X)$, где $X=\Sigma_{p} \vee \Sigma_{p}$, не сюръективно. Поскольку гомоморфизм Гуревича $h: \pi_{0}(X) \rightarrow$ $\widetilde{H}_{0}(X)$ сюръективен (см. заключительное замечание в $\left.\S 4\right)$, он переводит некоторый элемент $\pi_{0}(X)$ в $(\alpha+\mathbb{Z}, \alpha+\mathbb{Z}) \in\left(\mathbb{Z}_{p} / \mathbb{Z}\right) \oplus\left(\mathbb{Z}_{p} / \mathbb{Z}\right)$ для некоторого $\alpha \in \mathbb{Z}_{p} \backslash \mathbb{Z}$. $\mathrm{C}$ другой стороны, представитель всякого элемента $\stackrel{\Delta}{\pi}_{0}(X, b)$, где $b$ - отмеченная точка букета, имеет носитель в одном из двух сомножителей букета. Следовательно, $\stackrel{\Delta}{\pi}_{0}(X, b)$ отображается в (в действительности, на) подмножество $\left[\left(\mathbb{Z}_{p} / \mathbb{Z}\right) \oplus 0\right] \cup\left[0 \oplus\left(\mathbb{Z}_{p} / \mathbb{Z}\right)\right]$ группы $H_{0}(X, b)$.

ЗАМЕчАНИЕ. Всякий одномерный стинродовски связный компакт имеет шейп либо гавайской серьги, либо конечного букета окружностей, либо точки (А. Трыбулец; см. [29; 7.3.3]).

ПримеР 5.6 (гавайская серьга). Пусть $E$ - гавайская серьга, т. е. одноточечная компактификация $\mathbb{R} \times \mathbb{N}$. Тогда $\pi_{1}(E) \simeq \lim _{\longleftarrow} \pi_{1}\left(\bigvee_{i=1}^{r} S^{1}\right) \simeq \underset{\lim }{\longleftarrow} F_{r}$, где $F_{r}$ обозначает свободную группу $\left\langle x_{1}, \ldots, x_{r} \mid\right\rangle . \overleftarrow{\mathrm{C}}$ другой стороны, если $f:\left(S^{1}, \mathrm{pt}\right) \rightarrow(E, x)$ - отображение, где $x$ - компактифицирующая точка, то можно предполагать, что $f$ трансверсально каждой $x_{n}:=(0, n) \in \mathbb{R}^{n} \times \mathbb{N}$. Прообраз всех этих точек является оснащенным 0-подмногообразием $S^{1} \backslash x$ с компонентами, проиндексированными буквами $x_{1}^{ \pm 1}, x_{2}^{ \pm 1}, \ldots$ так, что каждая $x_{i}$ встречается только конечное количество раз. Аналогично обычной конструкции 
Понтрягина-Тома, легко проверяется, что $\stackrel{\Delta}{1}_{1}(E)$ изоморфна группе бордизмов таких оснащенных многообразий. Из этого вытекает, что $\stackrel{\Delta}{\pi}_{1}(E)$ изоморфна подгруппе $L$ обратного предела $\lim F_{r}$, состоящей из всех нитей $\left(\ldots, g_{2}, g_{1}\right)$ таких, что если $g_{i}$ написать как приведенные слова $w_{i}$ в своих свободных группах, то каждое $x_{j}$ встретится лишь ограниченное количество раз (т. е. не более $n(j)$ раз для некоторой функции $n(j))$ в каждом $w_{i}$. Значит, $\stackrel{\Delta}{\pi}_{1}(E) \stackrel{\Delta}{\rightarrow} \pi_{1}(E)$ имеет нетривиальное коядро.

Напротив, ясно, что абелианизация $\stackrel{\Delta}{H}_{1}(E)$ группы $L$ отображается сюръективно на $H_{1}(E) \simeq \prod_{i=1}^{\infty} \mathbb{Z}$. Оказывается, ядро этой сюръекции выделяется прямым слагаемым в $\stackrel{\Delta}{H}_{1}(E)$ и изоморфно $\bigoplus_{\mathfrak{c}} \mathbb{Q} \oplus \bigoplus_{p} A_{p}$, где $\mathfrak{c}-$ мощность континуума, $p$ пробегает все простые числа, а $A_{p}-p$-адическое пополнение $\bigoplus_{\mathfrak{c}} \mathbb{Z}_{p}$ [84]. Это установлено прямым вычислением абелианизации $L$.

Пример 5.7 (гавайская улитка). Пусть $E^{n}-n$-мерная гавайская серьга, рассматриваемая теперь как одноточечная компактификация пространства $\mathbb{R}^{n} \times \mathbb{Z}$. Пусть $\sigma_{n}: E^{n} \rightarrow E^{n}-$ гомеоморфизм, заданный сдвигом $\mathbb{Z} \rightarrow \mathbb{Z}$, $i \mapsto i+1$. Пусть $X$ - тор гомеоморфизма $\sigma_{n}$, причем $n \geqslant 2$. Из точной последовательности Милнора легко видеть, что $\pi_{n}(X) \simeq H_{n}(X)=0$. С другой стороны, если $\widetilde{X}$ - универсальное накрывающее $X$, легко видеть, что $\stackrel{\Delta}{\pi}_{n}(\tilde{X})$ содержит $\mathbb{Z}\left[\mathbb{Z}\right.$. Следовательно, $\stackrel{\Delta}{\pi}_{n}(X)$ не отображается инъективно в $\pi_{n}(X)$.

Приступая к вычислению $\stackrel{\Delta}{H}_{n}(X)$, сначала заметим, что по теореме Гуревича (доказанной в требуемой общности, например, в [14]) $\stackrel{\Delta}{H}_{n}\left(E^{n}\right) \simeq \Delta_{n}\left(E^{n}\right)$ при $n>1$, а по конструкции Понтрягина-Тома (см. пример 5.6 или доказательство теоремы 1.1 ; ср. [85]) $\stackrel{\Delta}{\pi}_{n}\left(E^{n}\right) \simeq \prod_{i=1}^{\infty} \mathbb{Z}$ при $n>1$. Тогда из точной последовательности пары $\left(X, E^{n}\right)$ получаем $\prod_{i=1}^{\infty} \mathbb{Z} \stackrel{\partial}{\rightarrow} \prod_{i=1}^{\infty} \mathbb{Z} \rightarrow \stackrel{\Delta}{H}_{n}(X) \rightarrow 0$, где граничное отображение $\partial$ задано формулой $1-t: \mathbb{Z}\left[\left[t^{ \pm 1}\right]\right] \rightarrow \mathbb{Z}\left[\left[t^{ \pm 1}\right]\right]$. Но это - изоморфизм (обратное отображение задается формулой $\left.1+t+t^{2}+\cdots\right)$, значит, $\stackrel{\Delta}{H}_{n}(X)=0$.

ПримеР 5.8 (гавайская улитка с перегородками). Пусть $X$ - тор гомеоморфизма $\sigma_{n-1}$ из предыдущего примера, причем $n \geqslant 3$. Рассмотрим локально $(n-1)$-связный компакт $Y$, полученный из $X$ присоединением маленьких $n$-дисков $D_{1}, D_{2}, \ldots$ Мы покажем, что $\check{\tau} \stackrel{\Delta}{\tau}: \stackrel{\Delta}{\pi}_{n}(Y) \rightarrow \check{\pi}_{n}(Y)$ не сюръективно. (Отметим, что, согласно доказанной ниже теореме $6.5, \check{\tau} \stackrel{\Delta}{\tau}: \stackrel{\Delta}{\pi}_{n}\left(Y^{+}\right) \rightarrow$ $\check{\pi}_{n}\left(Y^{+}\right)$сюръективно, где $Y^{+}$получено приклейкой к $Y$ двумерного диска вдоль орбиты компактифицирующей точки.)

Ясно, что $H_{n}(X) \simeq \check{H}_{n}(X) \simeq \mathbb{Z}$ и $\pi_{n}(X) \simeq \check{\pi}_{n}(X) \simeq \mathbb{Z}[\mathbb{Z}]$. Выберем некоторое $a \in \pi_{n}(X)$, образ которого в $H_{n}(X)$ нетривиален, и пусть $b \in \pi_{n}(Y)-$ образ $a$. Допустим, $b=\stackrel{\Delta}{\tau}([f])$ для некоторого сфероида $f: S^{n} \rightarrow Y$. Тогда $f$ поднимается в универсальное накрывающее $Y_{\infty}$. Так как $S^{n}$ компактна, то образ поднятия $f$ содержится в объединении некоторого конечного количества $r$ сдвинутых экземпляров фундаментальной области $F_{0}$ действия $\mathbb{Z}$ на $Y_{\infty}$. Пусть $f_{r+1}: S^{n} \rightarrow Y_{r+1}$ - поднятие $f$ в $(r+1)$-кратное циклическое накрывающее $Y$. 
Тогда $f_{r+1}\left(S^{n}\right)$ не пересекает некоторого сдвинутого экземпляра $F_{i}$ фундаментальной области действия $\mathbb{Z} /(r+1)$.

По теореме $3.15(\mathrm{~b}) \pi_{n}\left(X_{r+1}\right) \rightarrow \pi_{n}(X)$ - изоморфизм, где $X_{r+1}-(r+1)$ кратное циклическое накрывающее $X$. Значит, $a$ - образ некоторого $a_{r+1} \in$ $\pi_{n}\left(X_{r+1}\right)$. Образ $b_{r+1} \in \pi_{n}\left(Y_{r+1}\right)$ элемента $a_{r+1}$ представляется сдвинутым экземпляром $f_{r+1}$, поэтому образ $b_{r+1}$ в $\pi_{n}\left(Y_{r+1}, \mathrm{Cl}\left(Y_{r+1} \backslash F_{j}\right)\right)$ тривиален для некоторого сдвинутого экземпляра $F_{j}$ фундаментальной области. В частности, $b_{r+1}$ отображается тривиально в $H_{n}\left(Y_{r+1}, \mathrm{Cl}\left(Y_{r+1} \backslash F_{j}\right)\right)$. Пусть $F_{j}^{\prime}=F_{j} \cap X_{r+1}-$ соответствующая фундаментальная область накрытия $X_{r+1} \rightarrow X$. Поскольку $Y_{r+1} n$-мерно, то $H_{n}\left(X_{r+1}, \mathrm{Cl}\left(X_{r+1} \backslash F_{j}^{\prime}\right)\right) \rightarrow H_{n}\left(Y_{r+1}, \mathrm{Cl}\left(Y_{r+1} \backslash F_{j}\right)\right)$ инъективно. Так как $\mathrm{Cl}\left(X_{r+1} \backslash F_{j}^{\prime}\right)$ деформационно ретрагируется на экземпляр $(n-1)$-мерного компакта $E^{n-1}$, то $H_{n}\left(X_{r+1}\right) \rightarrow H_{n}\left(X_{r+1}, \mathrm{Cl}\left(X_{r+1} \backslash F_{j}^{\prime}\right)\right)$ инъективно. Значит, $a_{r+1}$ отображается тривиально в $H_{n}\left(X_{r+1}\right)$. Но тогда $а$ отображается тривиально и в $H_{n}(X)$, что противоречит нашему выбору $a$.

ЗАмечАнИЕ. Другим локально $(n-1)$-связным компактом $Y$, для которого $\check{\tau} \stackrel{\Delta}{\tau}: \stackrel{\pi}{\pi}_{n}(Y) \rightarrow \check{\pi}_{n}(Y)$ не сюръективно, является $Y=S^{1} \vee E^{n}$, где в качестве отмеченной точки гавайской серьги $E^{n}$ взята ее компактифицирующая точка. Это было замечено Дыдаком [67; пример 8.10] и Здравковской [86], о чем автор не подозревал при составлении примера 5.8. Здравковская также показала, что $\stackrel{\Delta}{n}_{n}(X) \rightarrow \pi_{n}(X)$ не сюръективно ни для какого компакта $X$, стинродовски гомотопически эквивалентного $Y$. Напротив, Ферри доказал, что всякий $\mathrm{UV}_{1}$-компакт стинродовски гомотопически эквивалентен такому компакту $X$, что $\stackrel{\Delta}{\tau}:[Z, X]^{\triangle} \rightarrow[Z, X]$ - биекция для всякого конечномерного компакта $Z[21]$. Его построение всегда приводит к бесконечномерному $X$.

\section{§6. Сравнение теорий}

Напомним, что компакт $X$ локально $n$-связен или удовлетворяет условию $\stackrel{\Delta}{\mathrm{C}}_{n}$, если любая окрестность $U$ всякой точки $x \in X$ содержит окрестность $V$ этой точки такую, что $\stackrel{\Delta}{\pi}_{i}(V) \rightarrow \stackrel{\Delta}{\pi}_{i}(U)$ тривиально для всех $i \leqslant n$. Говорят, что $X$ удовлетворяет условию $п л y-\stackrel{\mathrm{L}}{\mathrm{C}}_{n}$, если он удовлетворяет $\mathrm{L}_{n-1}$ и каждая точка $x \in X$ имеет окрестность $V$ такую, что отображение $\stackrel{\Delta}{\pi}_{n}(V) \rightarrow \stackrel{\Delta}{\pi}_{n}(X)$ тривиально.

Сингулярная фундаментальная группа $\stackrel{\Delta}{\pi}_{n}(X)$ может быть топологизирована аналогично $\pi_{n}(X)$, т. е. ее подмножество объявляется открытым, если оно является прообразом открытого подмножества $\check{\pi}_{n}(X)$; или, что эквивалентно, если оно является прообразом точки при гомоморфизме, индуцированном некоторым отображением $X$ в полиэдр. Заметим, что $\stackrel{\Delta}{\pi}_{n}(X)$ и $\pi_{n}(X)$, вообще говоря, нехаусдорфовы, в то время как $\check{\pi}_{n}(X)$ метризуема.

Теорема 6.1. Зафиксируем некоторое $n \geqslant 0$, и путь $X$ - компакт.

(а) Следующие условия эквивалентны:

$\stackrel{\mathrm{LC}}{n}_{n}: \quad X$ локально $n$-связен относительно $\stackrel{\Delta}{\pi}_{i}(\cdot) ;$

$\mathrm{LC}_{n}: X$ локально $n$-связен относительно $\pi_{i}(\cdot)$; 
$\check{L}_{n}: X$ локально $n$-связен относителъно $\check{\pi}_{i}(\cdot)$.

(b) Если $X$ удовлетворяет $\mathrm{LC}_{1}$ или $n=0$, то следующие условия также эквиваленты предъдущим:

$\mathrm{HLC}_{n}: \quad X$ локалъно п-связен относительно $\stackrel{\Delta}{H}_{i}(\cdot) ;$

$\mathrm{HLC}_{n}: X$ локально $n$-связен относительно $H_{i}(\cdot)$;

$\mathrm{HLC}_{n}: \quad X$ локалъно $n$-связен относителъно $\check{H}_{i}(\cdot)$.

(с) Если $X$ удовлетворяет $\stackrel{\Delta}{\mathrm{L}}_{n}$, mо $\stackrel{\Delta}{\pi}_{n}(X) \stackrel{\stackrel{\Delta}{\rightarrow}}{\rightarrow} \pi_{n}(X)$ u $\pi_{n}(X) \stackrel{\check{\tau}}{\rightarrow} \check{\pi}_{n}(X)$ биективны.

(d) Если $X$ удовлетворяет полу-L $\stackrel{\Delta}{\mathrm{C}}_{n}$, то $\stackrel{\Delta}{\pi}_{n}(X) \stackrel{\check{\tau} \stackrel{\Delta}{\longrightarrow}}{\longrightarrow} \check{\pi}_{n}(X)$ биективно.

(е) Если $X$ удовлетворяет $\stackrel{\Delta}{\mathrm{L}}_{n-1}$, то $\stackrel{\Delta}{\pi}_{n}(X) \stackrel{\check{\tau} \stackrel{\Delta}{\tau}}{\longrightarrow} \check{\pi}_{n}(X)$ есть отображение на плотное подмножество.

(f) $X$ удовлетворяет полу-L $\stackrel{\Delta}{\mathrm{C}}_{n}$, если и только если $X$ удовлетворяет $\stackrel{\Delta}{\mathrm{C}}_{n-1} u \stackrel{\Delta}{\pi}_{n}(X)$ дискретна.

(g) $X$ удовлетворяет полу-LC $\mathrm{LC}_{n} u \stackrel{\Delta^{*}}{n}(X) \stackrel{\stackrel{\Delta}{\tau}}{\rightarrow} \pi_{n}(X)$ сюрвективно, если $u$ только если $X$ удовлетворяет $\mathrm{LC}_{n-1} u \pi_{n}(X)$ дискретна.

(h) $X$ удовлетворяет полу-LС $\check{C}_{n}$, если и только если $X$ удовлетворяет $\mathrm{LC}_{n-1} u \check{\pi}_{n}(X)$ дискретна.

Бо́льшая часть теоремы 6.1 содержится в литературе. Гуревич доказал, что компакт $X$ удовлетворяет $\mathrm{HLC}_{n}$, а также $\mathrm{LC}_{1}$ тогда и только тогда, когда он удовлетворяет $\mathrm{L}_{n}$ [87]. Борсук доказал эквивалентную форму второго утверждения (c) [88]. Часть (d) была доказана Куперберг [75], а в предположении $\stackrel{\Delta}{\mathrm{C}}_{n}$ - также Богатым [74]. Дыдак доказал (е), и что если $X$ удовлетворяет $\stackrel{\Delta}{\mathrm{L}}_{n}$, то $\check{\pi}_{n}(X)$ дискретна [67; теорема 8.7]. Он устанавливает эти утверждения, заодно с обоими утверждениями пункта (c), модификацией доказательства своего про-группового варианта теоремы Смейла (см. теорему 3.15 (e)); этот подход, заключающийся, подобно спектральной последовательности Зимана, в глобальном анализе локальной информации, представляет собой содержательную альтернативу методам, использованным в данном параграфе.

Для ясности в доказательстве мы опускаем все упоминания о базисных точках и предоставляем читателю убедиться, что это не приводит к недоразумениям. Доказательства всех частей опираются на следующее построение.

Конструкция ФЕРри [21; с. 381]. Представим $X$ как предел обратной последовательности $P$ полиэдров и PL-отображений и будем использовать обозначения, введенные в начале $\S 2$. Триангулируем $P_{[0, \infty)}$ так, чтобы диаметры симплексов стремились к нулю при приближении к $X$. Предполагая, что $X$ удовлетворяет $\stackrel{\Delta}{\mathrm{C}}_{n}$, заключаем индукцией по $n$, что для каждого $\varepsilon>0$ существует $k$ такое, что $Y:=X \cup P_{[k, \infty)}^{(n+1)}$ ретрагируется на $X$ с помощью ретракции $r_{k}^{(n+1)}, \varepsilon$-близкой к тождественному отображению. Так как $r_{k}^{(n+1)}$ близка к тождественному отображению и $P_{[0, \infty]}$ удовлетворяет $\stackrel{\Delta}{\mathrm{C}}_{\infty}$, то для каждого $l$ 
существует $k$ такое, что $r_{k}^{(n+1)}$ гомотопна тождественному отображению посредством гомотопии $Y \times I \rightarrow P_{[l, \infty]}$, неподвижной на $X$. Если $X$ удовлетворяет только полу-L $\stackrel{\Delta}{C}_{n}$, то ограничение $r_{k}^{(n)}$ на $P_{[k, \infty)}^{(n)}$ по-прежнему продолжается до отображения $P_{[k, \infty)}^{(n+1)} \rightarrow X$, которое, однако, уже не близко к тождественному отображению на симплексах старшей размерности.

ДоКАЗАТЕЛЬСТво тЕОРемы 6.1. (е) Представим заданный $\alpha \in \check{\pi}_{n}(X)$ стинродовским сфероидом $\Phi: S^{n} \times[0, \infty) \rightarrow P_{[0, \infty)}$. Можно предполагать, что каждое $\varphi_{i}=\left.\Phi\right|_{S^{n} \times\{i\}}: S^{n} \rightarrow P_{i}$ идет в $n$-мерный остов. Если $X$ удовлетворяет $\stackrel{\Delta}{\mathrm{C}}_{n-1}$, то для каждого $l$ существует $k$ такое, что $r_{k}^{(n)}$ гомотопно тождественному отображению внутри $P_{[l, \infty]}$ и, следовательно, $\psi_{k}:=r_{k}^{(n)} \varphi_{k}: S^{n} \rightarrow X$ гомотопно $\varphi_{k}$ внутри $P_{[l, \infty]}$. Сильная деформационная ретракция $P_{[l, \infty]}$ на $P_{l}$ переводит эту гомотопию в гомотопию между $p_{l}^{\infty} \psi_{k}$ и $p_{l}^{k} \varphi_{k}$. Она также переводит $\left.\Phi\right|_{S^{n} \times[l, k]}$ в гомотопию между $p_{l}^{k} \varphi_{k}$ и $\varphi_{l}$. Значит, $\left[\varphi_{l}\right] \in \pi_{n}\left(P_{l}\right)$ - образ $\left[\psi_{k}\right] \in \stackrel{\Delta}{\pi}_{n}(X)$. Отсюда вытекает, что $\check{\tau} \stackrel{\Delta}{\tau}\left(\left[\psi_{k}\right]\right) \in \check{\pi}_{n}(X)$ сходятся к $\alpha$.

(d): Сюрбективность. Будем использовать обозначения из доказательства части (е). Если $X$ удовлетворяет полу-L $\stackrel{\Delta}{C}_{n}$, то каждое $\psi_{k}$ гомотопно $\psi_{k+1}$ посредством $\left.r_{k}^{(n+1)} \Phi\right|_{S^{n} \times[k, k+1]}$. Значит, все $\check{\tau} \stackrel{\Delta}{\tau}\left(\left[\psi_{k}\right]\right)$ равны. Так как $\check{\pi}_{n}(X)$ хаусдорфова, они также равняются своему пределу $\alpha$.

(d): Ингективность [21; с. 381]. Докажем, что $\stackrel{\pi}{n}_{n}(X) \stackrel{\check{\tau} \stackrel{\Delta}{\tau}}{\longrightarrow} \check{\pi}_{n}(X) \stackrel{p_{k}^{\infty}}{\longrightarrow} \pi_{n}\left(P_{k}\right)$ инъективно. Если задан сингулярный сфероид $\varphi: S^{n} \rightarrow X$ в ядре этой композиции, то он ограничивает нульгомотопию $\hat{\varphi}: B^{n+1} \rightarrow P_{[l, \infty]}$. По теореме о клеточной аппроксимации можно предполагать, что ее образ попадает в $(n+1)$-мерный остов (не считая $\partial B^{n+1}$, которая отображена в $\left.X\right)$. Тогда $r_{k}^{(n+1)} \hat{\varphi}$ - нульгомотопия сфероида $\varphi$.

(c) Следует как из пункта (d), теоремы 3.1 (b) и части 1) следующей леммы, так и из инъективности в (d), теоремы 3.1 (b) и части 2) следующей леммы.

Лемма 6.2. Зафиксируем некоторое $n \geqslant 0$, и пусть $X-$ компакт.

1) (Борсук [88]) Если $X$ удовлетворяет ${\stackrel{\Delta}{C_{n}}}_{n}$, то $\pi_{n}(X) \stackrel{\check{\tau}}{\rightarrow} \check{\pi}_{n}(X)$ ингективно.

2) Если $X$ удовлетворяет $\stackrel{\mathrm{LC}}{n}_{n}$, то $\stackrel{\Delta}{\pi}_{n}(X) \stackrel{\Delta}{\rightarrow} \pi_{n}(X)$ сюрвективно.

3) (Гуревич [87]) Если $X$ удовлетворяет $\mathrm{LC}_{n} u \stackrel{\Delta}{\mathrm{C}}_{n-1}$, mо $\stackrel{\Delta}{\pi}_{n}(X) \stackrel{\check{\tau} \stackrel{\Delta}{\tau}}{\longrightarrow} \check{\pi}_{n}(X)$ ингективно.

ДоказАТЕЛьство. 1) [21; с. 381] Пусть задано отображение $\varphi: S^{n+1} \rightarrow P_{k}$. Можно предполагать, что его образ лежит в $(n+1)$-мерном остове, и тогда $r_{k}^{(n+1)} \varphi: S^{n+1} \rightarrow X$ гомотопно $\varphi$ со значениями в $P_{[l, \infty)}$. Значит, $\stackrel{\Delta}{\pi}_{n+1}(X)$ отображается на образ $\pi_{n+1}\left(P_{k}\right)$ в $\pi_{n+1}\left(P_{l}\right)$. Отсюда вытекает, что $\pi_{n+1}\left(P_{i}\right)$ удовлетворяет условию Миттаг-Леффлера, и утверждение следует из теоремы $3.1(\mathrm{~b})$.

2 ) Для заданного стинродовского сфероида $\chi: S^{n} \times[0, \infty) \rightarrow P_{[0, \infty)}$ с образом в $(n+1)$-мерном остове каждое $t \in[k, \infty)$ доставляет сингулярный сфероид 
$\varphi_{t}=\left.r_{k}^{(n+1)} \chi\right|_{S^{n} \times\{t\}}: S^{n} \rightarrow X$. Образ $\left[\varphi_{k}\right]$ в $\pi_{n}(X)$ представляется отображением $\Phi_{k}^{+}: S^{n} \times[0, \infty) \rightarrow P_{[0, \infty)}$, определенным как $\Phi_{k}^{+}(x, t)=\Pi_{t}\left(\varphi_{k}(x)\right)$ (гомотопия $\Pi_{t}$ определена в начале $\left.\S 2\right)$. Семейство отображений $\Phi_{t}: S^{n} \times[t, \infty) \rightarrow$ $P_{[t, \infty)}$, определенное как $\Phi_{t}(x, s)=\Pi_{s}\left(\varphi_{t}(x)\right)$, доставляет собственную гомотопию между $\Phi_{k}$ и отображением $\Psi: S^{n} \times[k, \infty) \rightarrow P_{[k, \infty)}$, определенным как $\Psi(x, t)=\Phi_{t}(x, t)$. С другой стороны, то же семейство $\Phi_{t}$ доставляет гомотопию между $\Psi$ и $\left.r_{k}^{(n+1)} \chi\right|_{S^{n} \times[k, \infty)}$. Последнее гомотопно $\left.\chi\right|_{S^{n} \times[k, \infty)}$, так как $r_{k}^{(n+1)}$ гомотопно тождественному отображению. Результирующая гомотопия $h: S^{n} \times[k, \infty) \times I \rightarrow P_{[0, \infty]}$ между $\left.\chi\right|_{S^{n} \times[k, \infty)}$ и $\Psi$ такова, что $h^{-1}(C)-$ компакт для каждого компакта $C \subset P_{[0, \infty)}$. Поэтому ее можно снять с $X$ так, чтобы она стала собственной гомотопией внутри $P_{[k, \infty)}$. Результирующая собственная гомотопия между $\left.\chi\right|_{S^{n} \times[k, \infty)}$ и $\Phi_{k}$ может быть продолжена до собственной гомотопии между $\chi$ и $\Phi_{k}^{+}$с помощью леммы Борсука.

3) [89; §3]. Пусть $\varphi: S^{n} \rightarrow X-$ сингулярный сфероид, тривиальный по Чеху. Рассуждая, как в доказательстве инъективности в теореме $6.1(\mathrm{~d})$, получаем, что он ограничивает нульгомотопию $\Phi: B^{n+1} \rightarrow P_{[k, \infty]}$ для произвольного наперед заданного $k$. В частности, для заданного $\varepsilon>0$ мы можем выбрать $k$ так, чтобы $r_{k}^{(n)}$ было $\varepsilon$-близко к тождественному отображению и симплексы триангуляции $P_{[k, \infty]}$ имели диаметры $<\varepsilon$. В силу клеточной аппроксимации мы можем предполагать, что $\Phi$ переводит $n$-мерный остов $T^{(n)}$ некоторой триангуляции $T$ внутренности шара $B^{n+1}$ в $n$-мерный остов $P_{[k, \infty]}$ и, более того, что $\Phi(\partial \sigma)$ имеет диаметр $<3 \varepsilon$ для каждого $(n+1)$-симплекса $\sigma$ триангуляции $T$. Тогда образ ограничения $\varphi_{\sigma}: \partial \sigma \rightarrow X$ ретракции $r_{k}^{(n)} \varphi: T^{(n)} \rightarrow X$ имеет диаметр $<5 \varepsilon$. Более того, так как $P_{[k, \infty]}$ удовлетворяет $\stackrel{\Delta}{\mathrm{C}}_{\infty}$, то для произвольного заданного $\delta>0$ мы можем выбрать $\varepsilon$ так, чтобы каждое $\varphi_{\sigma}$ было $\delta$-нульгомотопно в $P_{[k, \infty]}$. Так как $X$ удовлетворяет $\mathrm{LC}_{n}$, то $\varphi_{\sigma}$ ограничивает $\delta$-нульгомотопию $\Phi_{\sigma}: \sigma \rightarrow P_{\left[k_{1}, \infty\right]}$ для произвольного наперед заданного $k_{1}$.

Теперь можно повторить весь процесс, с меньшим $\varepsilon$, отдельно для каждого $\varphi_{\sigma}: S^{n} \rightarrow X$, где $\sigma$ пробегает все $(n+1)$-симплексы $T$. Конструкция сходится к отображению $f$ в $X$, определенному на объединении $U n$-остовов $T_{i}^{(n)}$ последовательности последовательных измельчений $T_{i}$ триангуляции $T$ таких, что каждый $(n+1)$-симплекс $\Delta$ триангуляции $T_{i}$ имеет диаметр $<2^{-i}$ и $f(\partial \Delta)$ имеет диаметр $<2^{-i}$. Так как $U-$ плотное подмножество в $B^{n+1}$, это также определяет $f$ на всем $B^{n+1}$, и такое продолжение, как легко видеть, корректно определено и непрерывно.

ДоКАЗАТЕЛЬСТво тЕОРЕмы 6.1 (ПРОДолЖЕниЕ). (а) Если $X$ удовлетворяет $\stackrel{\Delta}{C}_{n}$, то по локальной версии 19 леммы $\left.6.2,2\right), X$ удовлетворяет $\mathrm{LC}_{n}$. (Отметим, что альтернативное доказательство утверждения 2) может быть получено из (d) и 1).) Если $X$ удовлетворяет $\mathrm{LC}_{n}$, то оно также удовлетворяет $\mathrm{LC}_{n}$, так

\footnotetext{
${ }^{19}$ Под "локальной версией $\mathfrak{X} "$ понимается "подходящее обобщение $\mathfrak{X}$ (доказываемое аналогично $\mathfrak{X})$, примененное к окрестностям точек". Так, например, сейчас речь идет о следующем обобщении леммы $6.2,2)$ : если $Z \subset Y \subset X$ - компакты, где $X$ удовлетворяет $\stackrel{\Delta}{C}_{n}$, а $Y-$ окрестность $Z$ в $X$, то образ $\stackrel{\Delta}{\tau}: \stackrel{\Delta}{\pi}_{n}(Y) \rightarrow \pi_{n}(Y)$ содержит образ $i_{*}: \pi_{n}(Z) \rightarrow \pi_{n}(Y)$. В некоторых случаях $\mathfrak{X}$ удается применить напрямую, используя теорему 6.11 .
} 
как $\check{\tau}$ - всегда эпиморфизм. Наконец, чтобы доказать, что LС $\check{\mathrm{C}}_{n}$ влечет $\stackrel{\Delta}{\mathrm{C}}_{n}$, мы можем предполагать, рассуждая по индукции, что $X$ удовлетворяет $\mathrm{L}_{n-1}$. Тогда утверждение следует из локальной версии леммы $6.2,3)$.

$(\mathrm{h}), \Rightarrow$. Если некоторый элемент группы $\pi_{n}(X)$ отображается тривиально в $\pi_{n}\left(P_{k}\right)$, то он может быть представлен сохраняющим уровни стинродовским сфероидом $\chi: S^{n} \times[0, \infty) \rightarrow P_{[0, \infty)}$, сужение которого на $S^{n} \times\{k\}$ ограничивает нульгомотопию $\varphi: D^{n+1} \rightarrow P_{k}$. Пусть $\psi: \mathbb{R}^{n+1} \rightarrow P_{[k, \infty)}$ - собственное отображение, полученное совмещением $\varphi$ с ограничением $\chi$ на $S^{n} \times[k, \infty)$. Мы можем предполагать, что $\psi$ клеточное по отношению к некоторой триангуляции $\mathbb{R}^{n+1}$. Так как $X$ удовлетворяет $\stackrel{\Delta}{C}_{n-1}$, то ретракция $r_{k}^{(n)}: Y \rightarrow X$ гомотопна тождественному отображению посредством гомотопии $h: Y \times I \rightarrow P_{[l, \infty]}$, неподвижной на $X$. Если $\Delta-(n+1)$-симплекс $\mathbb{R}^{n+1}$, пусть $\eta:(\partial \Delta) \times I \rightarrow P_{[l, \infty]}$ - ограничение $h$ на $\psi(\partial \Delta) \times I$. Применяя гомотопию $\Pi_{t}$ из начала $\S 2$, мы можем предполагать, что $\eta$ сужается до собственного отображения $(\partial \Delta) \times[0,1) \rightarrow P_{[l, \infty)}$. Так как $X$ удовлетворяет полу-L $\check{C}_{n}$, этот стинродовский сфероид тривиален по Чеху, если только $l$ достаточно велико. Поскольку $\Delta-$ произвольный, отсюда вытекает, что $\chi$ также тривиален по Чеху.

$(\mathrm{h}), \Leftarrow$. Согласно лемме $3.4(\mathrm{~b})$, из условия следует, что $\check{\pi}_{n}(X)$ отображается инъективно в $\pi_{n}\left(P_{k}\right)$ для некоторого $k$. С другой стороны, если $U \subset X$ столь мала, что ее образ в $P_{k}$ лежит в звезде некоторой вершины $P_{k}$, то композиция $\check{\pi}_{n}(U) \rightarrow \check{\pi}_{n}(X) \rightarrow \pi_{n}\left(P_{k}\right)$ тривиальна. Тогда и $\check{\pi}_{n}(U) \rightarrow \check{\pi}_{n}(X)$ должно быть тривиальным.

$(\mathrm{g}), \Rightarrow$. Для удобства обозначений предположим, что $n>0$; случай $n=0$ аналогичен. По условию любой элемент $\pi_{n}(X)$ представи́м сфероидом $\varphi$ : $S^{n} \rightarrow X$. Если композиция $S^{n} \stackrel{\varphi}{\longrightarrow} X \stackrel{p_{k}^{\infty}}{\longrightarrow} P_{k}$ нульгомотопна для некоторого $k$, то $\varphi$ ограничивает диск $\psi: D^{n+1} \rightarrow P_{[k, \infty)}$. Так как $X$ удовлетворяет $\stackrel{\Delta}{\mathrm{C}}_{n-1}$, то для любого заданного $\varepsilon>0$ первый шаг конструкции Гуревича (из доказательства леммы $6.2,1)$ ) представляет $[\psi] \in \stackrel{\Delta}{\pi}_{n}(X)$ в виде конечной суммы классов композиций $\left(S^{n}, \mathrm{pt}\right) \stackrel{p}{\rightarrow}\left(S^{n} \vee I, 0\right) \stackrel{f_{i}}{\longrightarrow}(X, x)$, где $p$ стягивает полусферу $S^{n}$ на вибриссу (whisker) $I$, отправляя базисную точку в конец вибриссы, и диаметр каждого $f_{i}\left(S^{n}\right)$ не превышает $\varepsilon$. Так как $X$ удовлетворяет полу-LC ${ }_{n}$, каждая из композиций стинродовски нульгомотопна. Таким образом, $\stackrel{\Delta}{\tau}([\varphi])=0$. Это доказывает, что композиция $\pi_{n}(X) \stackrel{\check{\tau}}{\longrightarrow} \check{\pi}_{n}(X) \stackrel{p_{k}^{\infty}}{\longrightarrow} \pi_{n}\left(P_{k}\right)$ инъективна для некоторого $k$, откуда и следует искомое утверждение.

$(\mathrm{g}), \Leftarrow$. По условию $\pi_{n}(X) \stackrel{\check{\tau}}{\rightarrow} \check{\pi}_{n}(X)-$ биекция, причем $\check{\pi}_{n}(X)$ дискретно. Тогда по (е) $\stackrel{\Delta}{\pi}_{n}(X) \stackrel{\stackrel{\Delta}{\tau}}{\rightarrow} \pi_{n}(X)$ сюръективно, а по (g) $X$ удовлетворяет полу-LC ${ }_{n}$.

(f) Если $X$ удовлетворяет полу-L $\stackrel{\Delta}{C}_{n}$, то $\check{\pi}_{n}(X)$ дискретна по доказательству инъективности в теореме $6.1(\mathrm{~d})$. (Иначе, из локальной версии (е), принимая во внимание, что $\check{\pi}_{n}(X)$ хаусдорфова, мы получаем, что $X$ удовлетворяет полу-LC $\check{C}_{n}$, и утверждение следует из (h).)

Обратно, если $\stackrel{\Delta}{n}_{n}(X)$ дискретна, то, по определению ее топологии, она отображается инъективно в $\check{\pi}_{n}(X)$; пусть $G_{n}$ обозначает образ этой инъекции. Если $n=0$, то, как множество, $\check{\pi}_{n}(X)$, очевидно, может быть отождествлено 
с множеством квазикомпонент $X$ (которое совпадает с множеством компонент, поскольку $X-$ компакт). Поэтому $G_{0}=\check{\pi}_{0}(X)$. Если $n>0$, то топологическая группа $\check{\pi}_{n}(X)$ может быть снабжена левоинвариантной метрикой, определенной как предел обратной последовательности метрик на $\pi_{n}\left(P_{i}\right)$, где расстояние между каждыми двумя точками равно 1. Так как индуцированная метрика на $G_{n}$ также левоинвариантна и индуцированная ей топология дискретна, можно заключить, что индуцированная ею равномерная структура дискретна. Значит, всякая последовательность Коши в $G_{n}$ постоянна начиная с некоторого момента, и, стало быть, $G_{n}$ замкнута в $\check{\pi}_{n}(X)$. Поэтому по пункту (е) $G_{n}=\check{\pi}_{n}(X)$, и мы заключаем, что $\check{\pi}_{n}(X)$ дискретна в обоих случаях $(n=0$ и $n>0)$. Тогда по пункту $(\mathrm{h}) X$ удовлетворяет полу-LC $\check{L}_{n}$ и, следовательно, также и полу-L $\stackrel{\Delta}{C}_{n}$.

(b) Если $X$ удовлетворяет $\stackrel{\Delta}{C}_{n}$, то $\stackrel{\Delta}{H}_{n}(X) \stackrel{\stackrel{\Delta}{\rightarrow}}{\rightarrow} H_{n}(X)$ и $H_{n}(X) \stackrel{\check{\tau}}{\rightarrow} \check{H}_{n}(X)$ суть изоморфизмы, что устанавливается аналогично доказательству (c), с использованием псевдомногообразий вместо $S^{n} \times[0, \infty)$. Если $X$ удовлетворяет $\stackrel{\Delta}{\mathrm{C}}_{n-1}$ и $\mathrm{HL}_{\mathrm{C}}$, это рассуждение по-прежнему работает: ретракция $r_{k}^{(n+1)}$ становится определенной "с точностью до присоединения ручек к симплексам старшей размерности", т. е. она теперь определена на $Y^{\prime}:=X \cup Q_{k}$, где $Q_{k}-$ полиэдр, полученный заменой каждого симплекса $\Delta^{n+1}$ старшей размерности в $P_{[k, \infty)}^{(n+1)}$ некоторым $(n+1)$-псевдомногообразием с краем $\partial \Delta^{n+1}$. Ретракция $r_{k}^{(n+1)}: Y^{\prime} \rightarrow X$ гомотопна проекции $Y^{\prime} \rightarrow Y$ (взамен $\operatorname{id}_{Y}$ ) посредством гомотопии $Y^{\prime} \times I \rightarrow P_{[l, \infty)}$, неподвижной на $X$.

Очевидно, что $\stackrel{\Delta}{C}_{0}$ эквивалентно HLС $\stackrel{\Delta}{0}_{0}$ и LС̆ $\check{C}_{0}$ эквивалентно HLC̆ $\check{C}_{0}$. Согласно вышесказанному, HLС $\stackrel{\Delta}{0}_{0}$ влечет $\mathrm{HLC}_{0}$, которое, в свою очередь, влечет HLС̆ Так как все сингулярные 1 -циклы - сферические, то $\stackrel{\Delta}{\mathrm{C}}_{1}$ влечет $\mathrm{HL}_{\mathrm{C}}$. Тогда, согласно вышесказанному, оно также влечет $\mathrm{HLC}_{1}$ и HLC $\check{C}_{1}$. Остается рассмотреть случай $n>1$. Рассуждая по индукции, мы можем предполагать, что $X$ удовлетворяет $\stackrel{\Delta}{\mathrm{C}}_{n-1}$. Тогда, по вышесказанному, $\mathrm{HLC}_{n}$ влечет $\mathrm{HLC}_{n}$, кото-

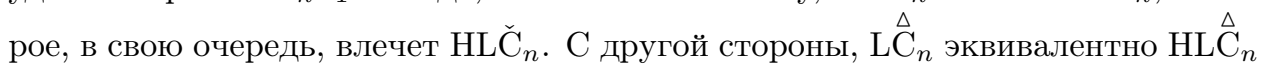
по лемме 6.3 (a) ниже, a L $\check{C}_{n}$ эквивалентно $\mathrm{HLC}_{n}$ по лемме 6.3 (b) ниже вместе с теоремой $6.1(\mathrm{~d})$.

Лемма 6.3. Пусть $f: X \rightarrow Y$ - отображение между компактами такое, что для любого отображения $g$ из $(n-1)$-полиэдра в $X$ композиция $f g$ нульгомотопна. Тогда:

(a) $\operatorname{im}\left[\stackrel{\Delta}{H}_{n}(X) \rightarrow \stackrel{\Delta}{H}_{n}(Y)\right]$ содержится в $\operatorname{im}\left[\Delta_{n}(Y) \rightarrow \stackrel{\Delta}{H}_{n}(Y)\right]$, и если $n>1$, mo $\operatorname{ker}\left[\Delta_{n}(X) \rightarrow \stackrel{\Delta}{H}_{n}(X)\right]$ содержится в $\operatorname{ker}\left[\stackrel{\Delta}{\pi}_{n}(X) \rightarrow \stackrel{\Delta}{\pi}_{n}(Y)\right]$;

(b) если $X$ удовлетворяет $\stackrel{\Delta}{\mathrm{C}}_{n-2} u n>1$, mо $\operatorname{im}\left[\check{H}_{n}(X) \rightarrow \check{H}_{n}(Y)\right] \subset$ $\operatorname{im}\left[\check{\pi}_{n}(Y) \rightarrow \check{H}_{n}(Y)\right] u \operatorname{ker}\left[\check{\pi}_{n}(X) \rightarrow \check{H}_{n}(X)\right] \subset \operatorname{ker}\left[\check{\pi}_{n}(X) \rightarrow \check{\pi}_{n}(Y)\right]$

Второе утверждение пункта (а) является, по существу, известной “теоремой Гуревича в конечном счете" ("Eventual Hurewicz Theorem"), сформулированной Ферри [90; 3.1] (название этой теоремы, произошедшее, по-видимому, из 
“Концов отображений - I" Ф. Квинна, утвердилось в литературе). Действительно, условие леммы 6.3 удовлетворено, если $f$ - композиция $f_{n-1} \cdots f_{0}$, где каждое $f_{i}$ тривиально на $\stackrel{\Delta}{\pi}_{i}$.

Из теоремы 6.7 будет следовать, что первое утверждение пункта (b) также выполнено при $n=1$, если предположить, что $X$ удовлетворяет $\stackrel{\Delta}{\mathrm{C}}_{0}$.

ДокАзАтЕльство. (а) Если задан сингулярный цикл $\varphi: Z \rightarrow X$, где $Z-$ $n$-псевдомногообразие с фиксированной триангуляцией, то по условию $f \varphi$ гомотопно такому $\psi$, которое отправляет $Z^{(n-1)}$ в базисную точку. Таким образом, $f_{*}[\varphi]=\sum\left[\psi_{i}\right]$, где $\psi_{i}: S^{n} \rightarrow Y$ доставляются ограничением $\psi$ на симплексы старшей размерности.

Если задана сингулярная $(n+1)$-цепь $\varphi: Z \rightarrow X$ с $\partial Z=S^{n}$, то по условию $f \varphi$ гомотопно такому $\psi$, которое отправляет $Z^{(n-1)}$ в базисную точку. Это $\psi$ пропускается через факторпространство $Z^{\prime}=Z / Z^{(n-1)}$, причем $\pi_{n}\left(Z^{\prime}\right) \rightarrow H_{n}\left(Z^{\prime}\right)$ является изоморфизмом по классической теореме Гуревича. Таким образом, $S^{n}=\partial Z$ нульгомотопна в $Z^{\prime}$ и, следовательно, в $Y$.

(b) Предположим, что $X$ и $Y$ - пределы обратных последовательностей компактных полиэдров $P_{i}$ и $Q_{i}$. Для каждого $l$ существует $k>l$ такое, что $r_{k}^{(n-1)}: X \cup P_{[k, \infty)} \rightarrow X$ определено и гомотопно, со значениями в $P_{[l, \infty]}$, тождественному отображению (см. конструкцию Ферри). Тогда включение $\varphi: K \rightarrow P_{k}$ $(n-1)$-мерного остова $K$ некоторой триангуляции полиэдра $P_{k}$ гомотопно, со значениями в $P_{[l, \infty]}$, некоторому $\psi: K \rightarrow X$. По условию $f \psi: K \rightarrow Y$ нульгомотопно. По леммам 2.1 и $2.5\left(\mathrm{~b}_{0}\right)$ мы можем предполагать (после отбрасывания некоторых $P_{i}$ ), что $f$ продолжается до сохраняющего уровни отображения $F: P_{[0, \infty]} \rightarrow Q_{[0, \infty]}$. Тогда $F \varphi$ нульгомотопно со значениями в $Q_{[l, \infty]}$. Значит, $\left.F\right|_{P_{k}}: P_{k} \rightarrow Q_{[l, \infty]}$ пропускается, с точностью до гомотопии, через $(n-1)$-связный полиэдр $L:=P_{k} / K$. По теореме Гуревича $\pi_{n}(L) \simeq H_{n}(L)$.

Рассмотрим стинродов сфероид $\varphi: S^{n} \times[0, \infty) \rightarrow P_{[0, \infty)}$ такой, что каждое $\varphi_{k}=\left.\varphi\right|_{S^{n} \times k}: S^{n} \rightarrow P_{k}$ представляет тривиальный элемент $H_{n}\left(P_{k}\right)$. Тогда $F \varphi_{k}$ представляет тривиальный элемент $\pi_{n}\left(Q_{[l, \infty]}\right)$. Поскольку $\varphi_{k}$ гомотопно, со значениями в $P_{[l, k]}$, отображению $\varphi_{l}$, то гомотопический класс $\left[F \varphi_{l}\right]$ также тривиален в $\pi_{n}\left(Q_{[l, \infty]}\right)$. Так как $Q_{[l, \infty]}$ деформационно ретрагируется на $Q_{l}$, то класс $\left[F \varphi_{l}\right]$ тривиален в $\pi_{n}\left(Q_{l}\right)$.

Пусть $\psi: M \rightarrow P_{[0, \infty)}-$ стинродовский цикл, и рассмотрим его уровни $\psi_{k}=$ $\left.\psi\right|_{\psi^{-1}\left(P_{k}\right)}$. Тогда $F \psi_{k}$ гомологично, со значениями в $Q_{[l, \infty]}$, сферическому циклу $\varphi_{l}: S^{n} \rightarrow Q_{[l, \infty]} \rightarrow Q_{l}$. Так как каждое $\psi_{k}$ гомологично $\psi_{k+1}$ со значениями в $P_{[k, k+1]}$, то каждое $\varphi_{l}$ гомотопно $\varphi_{l+1}$ со значениями в $Q_{[m+1, \infty]}$, где $l=l(m+1)$ выбирается аналогично $k=k(l)$. Тогда $\chi_{m}:=\Pi_{m} \varphi_{l}$ и $\chi_{m+1}$ гомотопны с сохранением уровней, со значениями в $Q_{[m, m+1]}$ (гомотопия $\Pi_{t}$ определена в $\S 2$ ). Таким образом, $\chi_{m}-$ уровни стинродовского сфероида $S^{n} \times[0, \infty) \rightarrow Q_{[0, \infty)}$. С другой стороны, так как $\psi_{m}$ гомологично $\psi_{k}$ со значениями в $P_{[m, k]}$ и $Q_{[m, \infty]}$ деформационно ретрагируется на $Q_{m}$, то композиция $F \psi_{m}$ гомологична $\chi_{m}$ со значениями в $Q_{m}$.

Сфероиды с хоботами. Будем называть $n$-сфероидом с хоботами в компакте $X$ всякое равномерно непрерывное отображение $\varphi:(\partial R, \mathrm{pt}) \rightarrow\left(X, x_{0}\right)$, где равномерное пространство $R$ является регулярной $\varepsilon$-окрестностью собствен- 
но вложенного бесконечного двоичного дерева в $\mathbb{R}^{n+1}$ по отношению к некоторой собственной функции $\varepsilon: \mathbb{R}^{n+1} \rightarrow(0,1]$. Заметим, что $\partial R$ неравномерно гомеоморфна дополнению до ручного канторова множества в $S^{n}$.

Если $X$ - предел обратной последовательности $P$ полиэдров и PL-отображений, то, аналогично доказательству леммы 2.1, каждый сфероид с хоботами $\varphi: \partial R \rightarrow X$ продолжается до равномерно непрерывного отображения $\bar{\Phi}: R \rightarrow P_{[0, \infty]}$, сужающегося до собственного отображения $\Phi: \operatorname{Int} R \rightarrow P_{[0, \infty)}$, и любые два такие продолжения гомотопны в классе таких продолжений. Будем говорить, что $\varphi$ представляет $[\Phi] \in \pi_{n}(X)$.

ПРЕДЛОЖЕНИЕ 6.4. Если компакт $X$ удовлетворяет $\mathrm{LC}_{n} u n \geqslant 0$, то любой элемент $\pi_{n+1}(X)$ представи́м $(n+1)$-сфероидом с хоботами.

ЗАмЕчАНИЕ. Предложение 6.4 может быть обобщено следующим образом. Если компакт $X$ удовлетворяет $\mathrm{LC}_{n}$, то каждый элемент $\pi_{n+d}(X)$ может быть представлен равномерно непрерывным отображением $\partial R \rightarrow X$, где $R$ - peгулярная $\varepsilon$-окрестность в $\mathbb{R}^{n+d+1}$ собственно вложенного телескопа обратной последовательности $(d-1)$-полиэдров $P_{i}$ (начиная с $P_{0}=\mathrm{pt}$ ) относительно некоторой собственной функции $\varepsilon: \mathbb{R}^{n+d+1} \rightarrow(0,1]$. В действительности, $P_{i}$ можно более точно описать как двойственные остовы последовательных барицентрических измельчений некоторой триангуляции сферы $S^{n+d}$, с очевидными связующими отображениями. В этом случае, если $2 d-1 \leqslant n+d$, то $\lim _{\longleftarrow} P_{i}$ го-

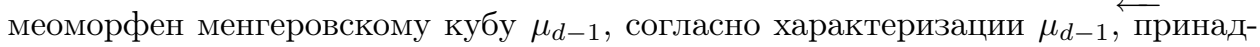
лежащей Бествине (см. [91]). Таким образом, $\partial R$ неравномерно гомеоморфна дополнению к ручному (по построению) экземпляру $\mu_{d-1}$ в сфере $S^{n+d}$.

ДокАЗАТЕЛЬство. Пусть $\Phi: S^{n+1} \times[0, \infty) \rightarrow P_{[0, \infty)}-$ стинродовский сфероид. Пусть $K^{[1]}, K^{[2]}, \ldots$ - последовательные барицентрических измельчения некоторой триангуляции $S^{n+1}$. (Строго говоря, чтобы получить в результате двоичное, а не $(n+1)$ !-арное дерево, нам следовало бы разложить каждую операцию барицентрического измельчения в последовательность дихотомических измельчений.) Пусть $L_{i}-n$-мерный остов $K^{[i]}$, и пусть $N_{i}$ - третья производная окрестность $L_{i}$ в $K^{[i]}$. Заметим, что $S^{n+1} \backslash \bigcup \operatorname{Int} N_{i}$ - ручное канторово множество $C$ и существует непрерывное отображение $\varphi: S^{n+1} \rightarrow S^{n+1}$, для которого $\varphi\left(\bigcup \operatorname{Int} N_{i}\right)=\bigcup L_{i}$ и $\varphi(C)=S^{n+1}$. (Оно строится аналогично отображению $[0,1] \rightarrow[0,1]$, которое конвертирует бесконечную троичную дробь $A=0 . \alpha_{1} \alpha_{2}$ в двоичную $B=0 . \beta_{1} \beta_{2} \ldots$, заменяя все двойки единицами и останавливаясь после первого появления единицы в $A$.) Пусть $N=\bigcup N_{i} \times[i, \infty) \subset S^{n+1} \times[0, \infty)$. С другой стороны, пусть $N^{\prime}-$ третья производная окрестность $\bigcup L_{i} \times[i, \infty)$ в $S^{n+1} \times[0, \infty)$. Пусть $E$ и $E^{\prime}-$ внешности $N$ и $N^{\prime}$ соответственно в $S^{n+1} \times[0, \infty)$.

Пусть $f: N^{\prime} \times[0,1] \rightarrow P_{[l, \infty]}$ - гомотопия между $\left.\Phi\right|_{N^{\prime}}$ и $\left.r_{k}^{(n+1)} \Phi\right|_{N^{\prime}}$. Используя гомотопию $\Pi_{t}$ из начала $\S 2$, мы можем предполагать, что $f^{-1}(X)=N^{\prime} \times\{1\}$. Пусть $h: S^{n+1} \times[0, \infty) \rightarrow E^{\prime} \cup \partial N^{\prime} \times[0,1)$ - гомеоморфизм такой, что $h(E)=E^{\prime}$. Пусть $\Phi^{\prime}$ определено как $\Phi h$ на $E$ и как $f h$ на $N$. Тогда $\Phi^{\prime}$ собственно гомотопно $\Phi$ (с помощью $f$ ) и продолжается по непрерывности до равномерно непрерывного отображения $\bar{\Phi}^{\prime}: S^{n+1} \times[0, \infty] \backslash C \times\{\infty\} \rightarrow P_{[0, \infty]}$. 
Теорема 6.5. Пусть $X$ - компакт, удовлетворяющий $\mathrm{LC}_{n-1}, u n \geqslant 1$.

(a) Eсли $\stackrel{\Delta}{\pi}_{1}(X)=1$, mо $\stackrel{\Delta}{\pi}_{n}(X) \stackrel{\stackrel{\Delta}{\tau}}{\rightarrow} \pi_{n}(X)$ - эпиморфизм.

(b) Если $\stackrel{\Delta}{\pi}_{1}(X)$ действует тривиально на $\stackrel{\Delta}{\pi}_{n}(X)$, mо $\stackrel{\Delta}{\pi}_{n}(X) \stackrel{\check{\tau} \stackrel{\Delta}{\longrightarrow}}{\longrightarrow} \check{\pi}_{n}(X)-$ эпиморфизм.

Заметим, что в случае $n \geqslant 2$ условия утверждений (а) и (b) могут быть ослаблены, с помощью теоремы $3.15(\mathrm{~b})$, до “ $\stackrel{\Delta}{1}_{1}(X)$ конечна" и "подгруппа конечного индекса в $\stackrel{\Delta}{\pi}_{1}(X)$ действует тривиально на $\stackrel{\Delta}{\pi}_{n}(X)$ " соответственно.

Необходимость односвязности в теореме 6.5 показана примером 5.8 .

ДоказАТЕЛЬСтво. Пусть $\varphi:(\partial R, \mathrm{pt}) \rightarrow(X, x)-n$-сфероид с хоботами, реализующий заданный элемент из $\pi_{n}(X)$ по предложению 6.4. Мы можем предполагать, что бесконечное двоичное дерево $T$, собственно вложенное в $\mathbb{R}^{n+1}$, является полным подкомплексом некоторой триангуляции $\mathbb{R}^{n+1}$, которую мы фиксируем с настоящего момента, и что $R$ является производной окрестностью $T$. Так как $X$ удовлетворяет $\mathrm{L}_{n-1}$, то $\varphi$ продолжается до отображения $\partial R \cup K \rightarrow X$, также обозначаемого $\varphi$, где $K$ - объединение двойственных клеток ко всем, за исключением конечного количества, граням $T$. Пусть $B_{0}, B_{1}, \ldots$ - замыкания компонент $R \backslash K$. Таким образом, все, кроме конечного количества, $B_{i}$ являются двойственными клетками вершин $T$, а остальные производными окрестностями конечных поддеревьев $T$. Без ограничения общности можно считать, что $B_{0}$ является производной окрестностью конечного поддерева $T_{1} \subset T$ и $B_{1}, B_{2}, \ldots$ - двойственные клетки вершин $T$. В частности, все $B_{i}$ являются PL-шарами.

Пусть $U_{i}=\left\{U_{i \alpha}\right\}$ - последовательность покрытий $X$ такая, что каждое $U_{i \alpha}$ имеет диаметр, не превышающий $2^{-i}$, и каждое $U_{i+1, \alpha}$ содержится в некотором $U_{i \beta}$. Без ограничения общности можно считать, что диаметр $X$ не превышает 1 , и, раз так, мы можем предполагать, что $U_{0}=\{X\}$. Тогда $T$ - объединение возрастающей цепи конечных (симплициальных) поддеревьев $T_{1} \subset T_{2} \subset$ $T_{3} \subset \cdots$ такой, что если $B_{j}$ - двойственная клетка некоторой вершины из $T \backslash T_{i}$, то $\varphi\left(\partial B_{j}\right)$ содержится в некотором $U_{i+1, \beta}$. Пусть $R_{i}$ - производная окрестность $T_{i}$, и пусть $B_{i 1}, \ldots, B_{i r_{i}}$ - двойственные клетки вершин $T_{i+1} \backslash T_{i}$ (где $\left.T_{0}=\varnothing\right)$. С помощью перенумерации и повторов элементов каждого $U_{i}$ несложно добиться, чтобы каждое $\varphi\left(\partial B_{i j}\right)$ лежало в $U_{i j}$. Пусть $\varphi_{0}: S^{n} \rightarrow x \in X-$ постоянное отображение. Предполагая, что $\varphi_{i}:\left(S^{n}, \mathrm{pt}\right) \rightarrow(X, x)$ определено, определим $\varphi_{i+1}$ присоединением каждого $\left.\varphi\right|_{\partial B_{i j}}$ к $\varphi_{i}$ вдоль некоторого пути в $U_{k l}$ из точки в $\varphi\left(\partial B_{i j}\right)$ в точку в $\varphi_{i}\left(S^{n}\right)$, где $U_{k l}$ содержит $U_{i j}$ и если $k<m<i$, то любое $U_{m p}$, содержащее $U_{i j}$, не пересекает $\varphi_{i}\left(S^{n}\right)$.

Так как $X-$ компакт, то $k=k(i) \rightarrow \infty$ при $i \rightarrow \infty$. Так как каждое $\varphi_{i+1}$ является $2^{-k(i)}$-близким к $\varphi_{i}$, то последовательность $\varphi_{1}, \varphi_{2}, \ldots$ равномерно сходится к непрерывному отображению $\varphi_{\infty}:\left(S^{n}, \mathrm{pt}\right) \rightarrow(X, x)$. Пусть $P_{i}-$ нерв покрытия $U_{i}$ (с опущенными повторяющимися элементами); зафиксируем симплициальные связующие отображения $P_{i+1} \rightarrow P_{i}$, возникающие благодаря тому, что $U_{i+1}$ вписано в $U_{i}$, и пусть $p_{i}^{\infty}: X \rightarrow P_{i}$ - проекция. Каждое $p_{i}^{\infty} \varphi_{\infty}:\left(S^{n}, \mathrm{pt}\right) \rightarrow\left(P_{i}, p_{i}\right)$ гомотопно $p_{i}^{\infty} \varphi_{i}$ "прямолинейной" гомотопией. 
Последовательные пары этих гомотопий доставляют гомотопии $h_{i}^{(1)}$ между $p_{i}^{\infty} \varphi_{i+1}$ и $p_{i}^{\infty} \varphi_{i} . \quad$ Представитель $\Phi: \operatorname{Int} R \rightarrow P_{[0, \infty)}$ класса $[\varphi]$, продолжающийся до равномерно непрерывного отображения $\bar{\Phi}: R \rightarrow P_{[0, \infty]}$, совпадающего с $\varphi$ на $\partial R$, может быть получен с помощью совмещения некоторых гомеоморфизмов $f_{i}:\left(S^{n}, \mathrm{pt}\right) \rightarrow\left(\partial R_{i}, \mathrm{pt}\right)$ с “прямолинейными" гомотопиями $h_{i}^{(2)}:\left(S^{n}, \mathrm{pt}\right) \times I \rightarrow\left(P_{[i, i+1]}, p_{[i, i+1]}\right)$ между отображениями $p_{i}^{\infty} \varphi f_{i+1}$ и $p_{i}^{\infty} \varphi f_{i}$.

По построению каждое $\varphi_{i}$ имеет вид $\left(\psi_{11}+\cdots+\psi_{1 r_{1}}\right)+\cdots+\left(\psi_{i 1}+\cdots+\psi_{i r_{i}}\right)$, где каждое $\psi_{i j}:\left(S^{n}, \mathrm{pt}\right) \rightarrow(X, x)$ свободно (т. е. непунктированно) гомотопно $\left.\varphi\right|_{\partial B_{i j}}$. Аналогично, каждое $\varphi f_{i}$ имеет вид $\left(\chi_{11}+\cdots+\chi_{1 r_{1}}\right)+\cdots+\left(\chi_{i 1}+\right.$ $\left.\cdots+\chi_{i r_{i}}\right)$, где каждое $\chi_{i j}:\left(S^{n}, \mathrm{pt}\right) \rightarrow(X, x)$ свободно гомотопно $\left.\varphi\right|_{\partial B_{i j}}$. Так как $\Delta_{1}(X)$ действует тривиально на $\stackrel{\Delta}{\pi}_{n+1}(X)$, то забывающее отображение $\stackrel{\Delta}{n}_{n}(X) \rightarrow$ $\left[S^{n}, X\right]^{\triangle}$ в множество свободных гомотопических классов является биекцией. Таким образом, каждое $\psi_{i j}$ (пунктированно) гомотопно $\chi_{i j}$ некоторой гомотопией $h_{i j}$, и, следовательно, каждое $\varphi_{i}$ гомотопно $\varphi f_{i}$ некоторой гомотопией $h_{i}$. Более того, если $\stackrel{\Delta}{\pi}_{1}(X)=1$, то гомотопии $h_{i j}$ могут быть взяты в классе отображений $\left(S^{n}, \mathrm{pt}\right) \rightarrow(Y, 0) \rightarrow(X, x)$ (где $Y$ - одноточечная компактификация $\left.\mathbb{R}^{n} \sqcup[0, \infty)\right)$ таких, что образ $\mathbb{R}^{n}$ содержится в $U_{i j}$. Значит, каждое отображение $S^{n+1} \rightarrow P_{i}$, заданное "прямолинейными" нульгомотопиями $p_{i}^{\infty} \psi_{i j}$ и $p_{i}^{\infty} \chi_{i j}$ наряду с гомотопией $p_{i}^{\infty} h_{i j}$, нульгомотопно ("прямолинейной" гомотопией). Отсюда вытекает, что каждое $p_{i}^{\infty} h_{i+1}$ гомотопно $p_{i}^{\infty} h_{i}$ "прямолинейной" гомотопией, продолжающей $h_{i}^{(1)}$ и $h_{i}^{(2)}$. Теорема 6.5 доказана.

Из теоремы 6.5 и теоремы 6.1 (g) вытекает следующее утверждение.

СледСтвиЕ 6.6. Пусть $X-$ компакт $с \stackrel{\Delta}{\pi}_{1}(X)=1$, и пусть $n \geqslant 1$. Компакт $X$ удовлетворяет условию полу-LC ${ }_{n}$ тогда и только тогда, когда $X$ удовлетворяет $\mathrm{LC}_{n-1} u \pi_{n}(X)$ дискретна.

Нульмерный аналог следствия 6.6 (без предположения односвязности) получен в $\S 8$ (см. следствие 8.8). Вслед за ним дан набросок альтернативного подхода к доказательству утверждения, близкого к следствию 6.6; автор надеется, что этот подход поможет пролить свет на вопрос о необходимости предположения односвязности в формулировке следствия 6.6.

Рассмотрим теперь гомологические версии вышеприведенных результатов.

Теорема 6.7 (Эда-Кавамура, Щепин). Пусть компакт X удовлетворяет условию $\mathrm{LC}_{n-1}$ u $n \geqslant 1$. Тогда $\stackrel{\Delta}{H}_{n}(X) \stackrel{\stackrel{\Delta}{\tau}}{\rightarrow} H_{n}(X)$ - эпиморфизм.

Случай $n=1$ был установлен Е. В. Щепиным [92; теорема 4.1], с использованием диссертации Бествины и других нетривиальных структурных результатов топологии компактов. Около пяти лет назад автор узнал от Щепина, что тот также может доказать общий случай теоремы 6.7; это доказательство не было записано. Следует отметить, что некоторые ключевые части рассуждения из [92], по-видимому, не обобщаются на случай произвольного $n$, и автору неизвестно, каким образом Щепину удалось преодолеть эти трудности. Эда и Кавамура [93] доказали такое следствие теоремы 6.7: если $X$ удовлетворяет $\mathrm{L}_{n-1}$ и $n \geqslant 1$, то $\stackrel{\Delta}{H}_{n}(X) \stackrel{\check{\tau} \stackrel{\Delta}{\tau}}{\longrightarrow} \check{H}_{n}(X)$ - эпиморфизм. 
Ограничение $n \geqslant 1$ необходимо в теореме 6.7, как показывает пример 5.4.

ДокАзАтельство. Аналогично предложению 6.4 можно показать, что если $X$ удовлетворяет $\stackrel{\Delta}{\mathrm{C}}_{n-1}$ (или даже только $\mathrm{HL} \stackrel{\Delta}{\mathrm{C}}_{n-1}$ ), то каждый элемент $H_{n}(X), n \geqslant 1$, может быть представлен равномерно непрерывным отображением $M \rightarrow X$ из ориентированного $n$-псевдомногообразия, допускающего собственное $\varepsilon$-отображение $M \rightarrow T$ (т. е. отображение, при котором прообразы точек имеют диаметры $<\varepsilon$ ) на бесконечное двоичное дерево, относительно некоторой собственной функции $\varepsilon: T \rightarrow(0,1]$. Так как $X$ удовлетворяет $\stackrel{\Delta}{\mathrm{C}}_{n-1}$ (или даже только $\mathrm{HL}_{\mathrm{C}} \stackrel{\Delta}{n-1}$ ), “каждый хобот" в этом "цикле с хоботами" может быть разложен в бесконечную сумму маленьких сингулярных циклов. Тогда по лемме 6.3 (а) условие $\stackrel{\mathrm{L}}{\mathrm{C}}_{n-1}$ позволяет заменить $M$ суммой сингулярного компактного псевдомногообразия $M_{0} \rightarrow X$ и конечным количеством сфероидов с хоботами $\partial R_{i} \rightarrow X$. Последние можно преобразовать в своих стинродовских гомологических классах (но не в своих стинродовских гомотопических классах!) в настоящие сфероиды $S^{n} \rightarrow X$, следуя доказательству теоремы 6.5 (которое упрощается, поскольку теперь не требуется следить за базисными точками).

Теорема 6.8. Зафиксируем некоторое целое $n \geqslant 0$, и пусть $X$-компакт.

(a) (Щепин) $\mathrm{HL}_{n} \Rightarrow \mathrm{HLC}_{n} \Leftrightarrow \mathrm{HLC}_{n}$.

(b) (Юссила [94]; см. также [12; 2-е изд., VI.10.6]) Если X удовлетворяет полу-HLC ${ }_{n}$, mо $H_{n}(X) \stackrel{\check{\tau}}{\rightarrow} \check{H}_{n}(X)-$ изоморбизм.

(c) Если $X$ удовлетворяет $\mathrm{HL}_{\mathrm{C}}, \stackrel{\Delta}{n}$, то $\stackrel{\Delta}{H}_{n}(X) \stackrel{\Delta}{\rightarrow} H_{n}(X)$ - изоморбизм.

(d) (Мардешич [95]; см. также [12; 2-е изд., VI.12.6]) Если X удовлетворяет полу-НL $\stackrel{\Delta}{\mathrm{C}}_{n}$, то композиция $\stackrel{\Delta}{H}_{n}(X) \stackrel{\check{\tau} \stackrel{\Delta}{\tau}}{\longrightarrow} \check{H}_{n}(X)$ - изоморфизм.

(е) Если $X$ удовлетворяет $\mathrm{HL}_{n-1}$, mо $\stackrel{\Delta}{H}_{n}(X) \stackrel{\check{\tau} \check{t}}{\longrightarrow} \check{H}_{n}(X)-$ сюргекция на плотное подмножество.

(f) $X$ удовлетворяет полу-НL $\stackrel{\Delta}{\mathrm{C}}_{n}$, если и только если $X$ удовлетворяет $\mathrm{HL}_{\text {C }}$ u $u \stackrel{\Delta}{H}_{n}(X)$ дискретна.

(g) $X$ удовлетворяет полу-НLC ${ }_{n}$, если и только если $X$ удовлетворяет $\mathrm{HLC}_{n-1}$ и $H_{n}(X)$ дискретна.

(h) $X$ удовлетворяет полу-НLС $\check{C}_{n}$, если и только если X удовлетворяет $\mathrm{HLC}_{n-1} u \check{H}_{n}(X)$ дискретна.

Часть (g) следует непосредственно из (a), (b) и (h). Часть (c) следует из (a), (b) и (d) (cp. [12; 2-е изд., следствие V.12.15 и 1-е изд., следствие 5.11.9]), но мы также дадим прямое доказательство.

Часть (а) была доказана Щепиным [92; теорема 4.10] в случае $n=1$. Около пяти лет назад автор узнал от Щепина, что тот также может доказать общий случай (а); это доказательство не было записано. Приводимое доказательство (а) основано на той же идее, что и рассуждение из [92], а именно, на конструкции Гуревича (см. доказательство леммы $6.2,3)$ ); однако воплощение этой идеи в случае высоких размерностей опирается на более тонкую 
технику геометрического подхода к когомологиям, развитого Буонкристиано, Рурком и Сандерсоном [96].

Импликация $\mathrm{HLC}_{\infty} \Rightarrow \mathrm{HLC}_{\infty}$ не может быть обращена для грозди экземпляров произвольного неодносвязного ацикличного 2-полиэдра [97].

Псевдокомногообразия. Мы называем собственное кусочно линейное отображение $f: W \rightarrow P$ между полиэдрами (косвязным) $k$-псевдокомногообразием в $P$, если существует триангуляция $P$ такая, что прообраз $f^{-1}\left(\Delta^{i}\right)$ каждого ее $i$-симплекса является (псевдосвязным) $(i-k)$-псевдомногообразием с краем, равным прообразу края $f^{-1}\left(\partial \Delta^{i}\right)$. Такая триангуляция $P$ будет называться трансверсальной к $f$. Комногообразия появились в книге [96], где они называются "mock bundles".

Вложенное $k$-псевдокомногообразие $f: W \hookrightarrow P$ называется коориентируемъмм, если группа $H_{k}(P, P \backslash W)$ не содержит кручения. В этом случае группа $H^{k}(P, P \backslash W)$ - свободная абелева, и выбор набора ее образующих, представимых коциклами с дизъюнктными носителями, называется коориентацией $f$. (Для сравнения, $k$-псевдомногообразие $M$ без края ориентируемо, если и только если когомологии с компактным носителем $H_{\mathrm{c}}^{k}(M)$ не содержат кручения; в этом случае локально-конечные гомологии $H_{k}^{\mathrm{lf}}(M)$ - свободная абелева группа, и выбор набора ее образующих, представимых циклами с дизъюнктными носителями, соответствует ориентации $M$.) Если $f: W \rightarrow P$ - произвольное псевдокомногообразие, где полиэдр $P$ компактен, то оно является проекцией вложенного псевдокомногообразия $\bar{f}: W \hookrightarrow P \times \mathbb{R}^{N}$ для некоторого $N$, и коориентированность (коориентацию) $f$ можно корректно определить как коориентированность (коориентацию) $\bar{f}$.

Легко видеть, что $H^{k}(P ; \mathbb{Z} / 2)$ (соответственно $\left.H^{k}(P)\right)$ изоморфна группе классов (ориентированных) псевдокобордизмов (ориентированных) $k$-псевдокомногообразий в $P$, ср. [96].

Пусть $f: W \rightarrow P$ - ориентированное $k$-псевдокомногообразие. Заметим, что если $P$ - ориентированное $i$-псевдомногообразие по отношению к некоторой триангуляции $P$, трансверсальной к $f$, то $W$ будет ориентированным $(i-k)$-псевдомногообразием, см. [96; II.1.2]. С другой стороны, если $\varphi: M \rightarrow P$ симплициально относительно некоторой триангуляции $P$, трансверсальной к $f$, то пулбек (т. е. обратный образ) $\varphi^{*}(f): N \rightarrow M$ будет ориентированным $k$-псевдокомногообразием, см. [96; с. 23, внизу]. Если же $\varphi$ оказалось ориентированным сингулярным $i$-псевдомногообразием, мы получаем ориентированное сингулярное $(i-k)$-псевдомногообразие $f^{!}(\varphi): N \rightarrow W$. Используя технику [96], легко показать, что трансфер $f^{!}: H_{i}(P) \rightarrow H_{i-k}(W)$ корректно определен. Более того, $f_{*} f^{!}[\varphi]=[\varphi] \frown[f]$, см. [96; с. 29]. Сказанное иллюстрирует следующая диаграмма:

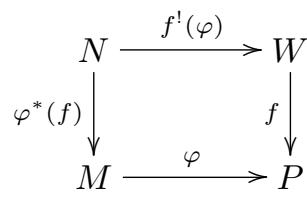

Если $f: W \rightarrow P$ - косвязное ориентированное 0-псевдокомногообразие, легко видеть, что $[f]= \pm\left[\operatorname{id}_{P}\right] \in H^{0}(P)$. Будем называть такое $f$ доработкой по- 
лиэдра $P$, если в точности $[f]=\left[\operatorname{id}_{P}\right] \in H^{0}(P)$. Тогда, в частности, $f_{*} f^{!}=$ $\mathrm{id}: H_{i}(P) \rightarrow H_{i}(P)$ для каждого $i$. Поэтому $H_{i}(P)$ можно отождествить с прямым слагаемым в $H_{i}(W)$. Кроме того, используя еще раз косвязность $f$, получим, что для каждого ориентированного сингулярного $i$-псевдомногообразия $\varphi: M \rightarrow P$, симплициального относительно триангуляции $P$ и трансверсального $f$, доработка $\varphi^{*}(f): N \rightarrow M$ индуцирует изоморфизм $H_{i}(N) \rightarrow H_{i}(M)$ в силу псевдосвязности $N$.

ДоРАБОТАННАЯ КОНСТРУКцИЯ ФЕРРИ. Предполагая, что $X$ удовлетворяет $\mathrm{HL}_{n} \stackrel{\Delta}{\mathrm{C}}_{n}$, мы заменим ретракцию $r_{k}^{(n+1)}: P_{[k, \infty)}^{(n+1)} \cup X \rightarrow X$ и ее гомотопию тождественному отображению со значениями в $P_{[l, \infty]}$, неподвижную на $X$ (см. “конструкцию Ферри” в начале настоящего параграфа), следующими данными:

(i) доработкой $q^{(n+1)}: Q^{n+1} \rightarrow P_{[k, \infty)}^{(n+1)}$;

(ii) ретракцией $\bar{r}_{k}^{(n+1)}: Q^{n+1} \cup X \rightarrow X$;

(iii) псевдокобордизмом $W^{n+2} \rightarrow P_{[k, \infty)}^{(n+1)}$ между $q^{(n+1)}$ и $\operatorname{id}_{P_{[k, \infty)}^{(n+1)}}$;

(iv) отображением $W^{n+2} \cup X \rightarrow P_{[l, \infty]}$, сужающимся в $\bar{r}_{k}^{(n+1)}$ на $Q^{n+1} \cup X$ и во включение на $P_{[k, \infty)}^{(n+1)}$.

Эти данные строятся аналогично конструкции Ферри, причем пункт (iii) возникает из определения доработки.

Если $X$ удовлетворяет полу-НL $\stackrel{\Delta}{C}_{n}$, то $\bar{r}_{k}^{(n)}: Q^{n} \cup X \rightarrow X$, ограниченное на $Q^{n}$, продолжается до непрерывного отображения $Q^{n+1} \rightarrow X$.

ДОКАЗАТЕЛЬСтво тЕОРЕмы 6.8 (c), (d), (е). Эти части могут быть доказаны аналогично соответствующим частям теоремы 6.1 с использованием доработанной конструкции Ферри.

Фрактализованные псевдомногообразия. Назовем обратную последовательность $\cdots \stackrel{f_{1}}{\longrightarrow} P_{1} \stackrel{f_{0}}{\longrightarrow} P_{0}$ полиэдров и кусочно линейных отображений фрактализующей, если каждое $f_{i}$ является доработкой, трансверсальной к триангуляции $T_{i}$ полиэдра $P_{i}$, такой, что $f_{i}$ симплициально по отношению к $T_{i+1}$ и некоторому измельчению барицентрического измельчения $T_{i}$. Такую последовательность триангуляций $\left(T_{0}, T_{1}, \ldots\right)$ будем также называть фрактализующей. Предел $F$ такой обратной последовательности - фрактализованный $P_{0}$, а $p_{0}^{\infty}: F \rightarrow P_{0}-$ фрактализация $P_{0}$.

Если $Q-n$-псевдомногообразие и $f: M \rightarrow Q$ - его фрактализация, то в силу сказанного $f_{*}: H_{n}(M) \rightarrow H_{n}(Q)$ - изоморфизм и, в частности, $M$ имеет корректно определенный фундаментальный класс $[M]$ в гомологиях Стинрода. Если $X$ - компакт и $\varphi: M \rightarrow X$ - отображение, мы говорим, что оно представляет $\varphi_{*}([M]) \in H_{n}(X)$.

Лемма 6.9. Пусть компакт $X$ удовлетворяет $\mathrm{HLC̆}_{n}$, u nусть $\varphi: M \rightarrow X-$ сингулярное фрактализованное ориентированное $n$-псевдомногообразие. Если $\check{\tau}([\varphi])=0 \in \check{H}_{n}(X)$, то $\varphi$ ограничивает сингулярный фрактализованный ориентированный нуль-псевдобордизм.

Идея доказательства основана на конструкции Гуревича (см. доказательство леммы $6.2,3))$. Случай $n=1$ был получен Щепиным [92; теорема 4.4]. Этот 
случай особ тем, что двумерные фрактальные псевдомногообразия сводятся к двумерным фрактальным многообразиям, которые все гомеоморфны друг другу, см. [92].

ДокАЗАТЕльство. Мы можем предполагать, что утверждение справедливо в размерностях $<n$. Предположим, что $M-$ предел фрактализующей обратной последовательности $\cdots \stackrel{f_{1}}{\longrightarrow} Q_{1} \stackrel{f_{0}}{\longrightarrow} Q_{0}$, где $Q_{0}$ - ориентированное $n$-псевдомногообразие. Предположим, что $X$ - предел обратной последовательности полиэдров $P_{i}$ и PL-отображений $p_{i}$. Без ограничения общности $\varphi$ продолжается до сохраняющего уровни отображения $\varphi_{[0, \infty]}: Q_{[0, \infty]} \rightarrow P_{[0, \infty]}$ (см. $\S 2$ ). Допуская вольность речи, переопределим $Q_{[0, \infty]}$, заменяя каждый цилиндр $M C\left(f_{i}\right)$ псевдокобордизмом между доработкой $f_{i}: Q_{i+1} \rightarrow Q_{i}$ и тождественным отображением $Q_{i}$. Тогда $\varphi_{[0, \infty)}$ - стинродовский цикл, представляющий $[\varphi]$, значит, по условию каждое $\varphi_{i}$ ограничивает сингулярное ориентированное псевдомногообразие $\hat{\varphi}_{i}: \widehat{Q}_{i} \rightarrow P_{i}$. Для заданного $\varepsilon>0$ существует $k$ такое, что найдется триангуляция пары $\left(\widehat{Q}_{k}, Q_{k}\right)$, продолжающая некоторую триангуляцию полиэдра $Q_{k}$ из фрактализующей последовательности триангуляций и такая, что образ каждого ее симплекса при отображении $\hat{\varphi}_{k}$ является $\varepsilon$-близким к $X$ и имеет диаметр, не превышающий $\varepsilon$. Заметим, что для каждого симплекса $\Delta$ этой триангуляции $Q_{k}$ обратная последовательность $\cdots \rightarrow\left(f_{k}^{k+2}\right)^{-1}(\Delta) \rightarrow$ $\left(f_{k}^{k+1}\right)^{-1}(\Delta) \rightarrow \Delta$ является фрактализующей. Тогда, аналогично конструкции Ферри (см. доказательство теоремы 6.1), из предположения индукции следует, что для каждого $\delta>0$ число $\varepsilon$ может быть выбрано так, что $\varphi_{[k+1, \infty]}$ продолжается до сохраняющего уровни отображения $\psi_{[k+1, \infty]}: R_{[k+1, \infty]} \rightarrow P_{[k+1, \infty]}$, где обратная последовательность $\cdots \stackrel{g_{k+1}}{\longrightarrow} R_{k+1} \stackrel{g_{k}}{\longrightarrow} R_{k}:=Q_{k} \cup \widehat{Q}_{k}^{(n)}$ является фрактализующей, каждое $g_{i}^{-1}\left(Q_{i}\right) \subset R_{i+1}$ отождествлено с $Q_{i+1}$ так, что $\left.g_{i}\right|_{Q_{i+1}}$ отождествлено с $\left.f_{i}\right|_{Q_{i+1}}$, и $\psi_{[k+1, \infty]}$ является $\delta$-близким к композиции проекции на $R_{k}$ и ограничения $\hat{\varphi}_{k}$. (Здесь $R_{[k+1, \infty]}$ состоит из псевдокобордизмов, а не цилиндров связующих отображений.)

Пусть $\Delta-(n+1)$-симплекс $\widehat{Q}_{k}$, и пусть $S_{i}=\left(g_{k}^{i}\right)^{-1}(\partial \Delta) \subset R_{i}$ для каждого $i>k$. Пусть $S_{\infty}-$ предел фрактализующей обратной последовательности $\cdots \rightarrow S_{k+1} \rightarrow S_{k}$. Тогда ограничение $\chi_{[k+1, \infty)}$ отображения $\psi_{[k+1, \infty)}$ на $S_{[k+1, \infty)}$ - стинродовский цикл, представляющий образ фундаментального класса $S_{\infty}$ в $H_{n}(X)$. Вспомним, что образ $S_{\infty}$ в $X$ имеет диаметр, не превышающий $\delta$. Так как $X$ удовлетворяет $\mathrm{HLC}_{n}$, то для каждого $\gamma>0$ это $\delta$ может тем самым быть выбрано так, чтобы $\chi_{[k+1, \infty)}$ был тривиально по Чеху с носителем в $\gamma$-окрестности образа $S_{\infty}$ в $X$.

Тогда каждое $\chi_{i}$ ограничивает сингулярное связное ориентированное псевдомногообразие $\hat{\chi}_{i}: \widehat{S}_{i} \rightarrow P_{i}$ с образом в $\gamma$-окрестности $\chi_{i}\left(S_{i}\right)$. Для любого конкретного значения $i$ ограничение $S_{i} \rightarrow \partial \Delta$ отображения $g_{k}^{i}$ можно продолжить до отображения $\widehat{S}_{i} \rightarrow \Delta$, симплициального по отношению к некоторому измельчению барицентрического измельчения $\Delta$. Повторяя то же для каждого $(n+1)$-симплекса $\Delta$ триангуляции $\widehat{Q}_{k}$, мы получим продолжение $g_{k}^{i}: R_{i} \rightarrow Q_{k} \cup \widehat{Q}_{k}^{(n)}$ до доработки $\hat{g}_{k}^{i}: \widehat{R}_{i} \rightarrow \widehat{Q}_{k}$ такой, что различные $\hat{\chi}_{i}$ совмещаются в отображение $\hat{\psi}_{i}: \widehat{R}_{i} \rightarrow P_{j}$. Так как $\hat{\psi}_{i}$ и $\hat{\varphi}_{k} \hat{g}_{k}^{i}$ являются $\gamma$-близкими, то для любого заданного $l$ мы можем выбрать $\gamma$ так, чтобы они продолжались до 
отображения $\hat{\psi}_{[k, i]}: \widehat{R}_{[k, i]} \rightarrow P_{[l, i]}$ псевдокобордизма $\hat{g}_{k}^{[i, k]}: \widehat{R}_{[k, i]} \rightarrow \widehat{Q}_{k}$ между $\hat{g}_{k}^{i}$ и тождественным отображением $\widehat{Q}_{k}$, которое сужается до $\psi_{[k, i]}: R_{[k, i]} \rightarrow P_{[k, i]}$.

Теперь, для любого заданного $l^{\prime}$, мы имеем аналогичный ряд зависимостей $l^{\prime} \mapsto \gamma^{\prime} \mapsto \delta^{\prime} \mapsto \varepsilon^{\prime} \mapsto k^{\prime}$, и вышеописанная процедура применима к $\hat{\chi}_{i}$ с $i=k^{\prime}$ вместо $\hat{\psi}_{k}$. Действуя таким образом, в конечном счете получим фрактализующую обратную последовательность $\left(\cdots \rightarrow T_{1} \rightarrow T_{0}\right)=(\cdots \rightarrow$ $\left.\widehat{R}_{i} \rightarrow \widehat{Q}_{k}\right)$ и собственное отображение $T_{[0, \infty)} \rightarrow P_{[l, \infty)}$, которое, по построению, продолжается до непрерывного отображения обратного предела $T_{\infty} \rightarrow X$, что доставляет требуемый сингулярный фрактализованный ориентированный нуль-псевдобордизм для $\varphi$. Лемма 6.9 доказана.

ФРАКТАЛИзОВАННАЯ КОНСТРУКция ФЕРРИ. Предполагая, что компакт $X$ удовлетворяет $\mathrm{HLC}_{n}$, лемму 6.9 можно применить для того, чтобы построить:

(i) фрактализующую обратную последовательность $\cdots \stackrel{q_{1}}{\longrightarrow} Q_{1} \stackrel{q_{0}}{\longrightarrow} Q_{0}=$ $P_{[k, \infty)}^{(n+1)}$ с обратным пределом $Q_{\infty}^{n+1}$;

(ii) ретракцию $\tilde{r}_{k}^{(n+1)}: Q_{\infty} \cup X \rightarrow X$;

(iii) “телескопический псевдокобордизм" $q_{0}^{[0, \infty]}: W_{[0, \infty]} \rightarrow P_{[k, \infty)}^{(n+1)}$, совмещающий псевдокобордизмы $q_{i}^{[i, i+1]}: W_{[i, i+1]} \rightarrow Q_{i}$ между $q_{i}$ и $\operatorname{id}_{Q_{i}}$ (таким образом, $\left.W_{\mathbb{N} \cup \infty}=Q_{\mathbb{N} \cup \infty}\right)$

(iv) "телескопическую гомотопию" $h_{k}^{(n+2)}: W_{[0, \infty]} \cup X \rightarrow P_{[l, \infty]}$, сужающуюся в $\tilde{r}_{k}^{(n+1)}$ на $Q_{\infty} \cup X$ и во включение на $P_{[k, \infty)}^{(n+1)}$.

СлЕДСтвие 6.10 (Е.В. Щепин). Если компакт X удовлетворяет $\mathrm{HLC}_{n}$, то каждый элемент $H_{n}(X)$ представи́м сингулярным фрактализованным ориентированным п-псевдомногообразием.

Это следует из фрактализованной конструкции Ферри аналогично доказательству леммы $6.2,2)$. Около пяти лет назад автор узнал от Е. В. Щепина, что тот может доказать некоторый вариант следствия 6.10 , используя другое (быть может, отличное) понятие фрактализованного псевдомногообразия, определенное прямым индуктивным построением.

ПРОДОЛЖЕНИЕ ДОКАЗАТЕЛЬСТВА ТЕОРЕМЫ 6.8. (а) ИМПликация $\mathrm{HLC}_{n} \Rightarrow$ $\mathrm{HLC}_{n}$ тривиальна. Обратная импликация следует из локальных версий следствия 6.10 и леммы 6.9. Импликация $\mathrm{HL}_{n} \stackrel{\Delta}{C}_{n} \operatorname{HLC}_{n}$ следует из $(\mathrm{d})$, или, иначе, импликация $\mathrm{HLC}_{n} \Rightarrow \mathrm{HLC}_{n}$ следует из (c).

(b) Доказательство похоже на доказательство следствия 6.6, но проще.

Представим заданный элемент группы $H_{n}(X)$ собственным сингулярным ориентированным $(n+1)$-псевдомногообразием $\varphi_{[0, \infty)}: Q_{[0, \infty)} \rightarrow P_{[0, \infty)}$. (Здесь каждое $Q_{[i, i+1]}$ - произвольный ориентированный псевдобордизм, а не цилиндр связующего отображения.) Если образ класса $\left[\varphi_{[0, \infty)}\right]$ при композиции

$$
H_{n}(X) \stackrel{\check{\tau}}{\longrightarrow} \check{H}_{n}(X) \stackrel{p_{k}^{\infty}}{\longrightarrow} H_{n}\left(P_{k}\right)
$$


тривиален, то мы можем предполагать, что $\varphi_{k}: Q_{k} \rightarrow P_{k}$ ограничивает сингулярное ориентированное $(n+1)$-псевдомногообразие $\hat{\varphi}_{k}: \widehat{Q}_{k} \rightarrow P_{k}$. Без ограничения общности стинродовский цикл $\varphi_{[k, \infty)} \cup \hat{\varphi}_{k}: Q_{[k, \infty)} \cup \widehat{Q}_{k} \rightarrow P_{[0, \infty)}$ отображается симплициально в $P_{[k, \infty)}^{(n+1)}$. Введем обозначения $Q=Q_{[k, \infty)} \cup \widehat{Q}_{k}$ и $\pi=\varphi_{[k, \infty)} \cup \hat{\varphi}_{k}: Q \rightarrow P_{[0, \infty)}$. Для заданного $\varepsilon>0$ мы можем выбрать $k$ так, что применима фрактализованная конструкция Ферри с $\tilde{r}_{k}^{(n)}$, переводящим $\left(q_{0}^{\infty}\right)^{-1}(\partial \Delta)$, для каждого $(n+1)$-симплекса $\Delta$ из $P_{[0, \infty)}$, в подмножество $X$ диаметра $<\varepsilon$. Для заданного $(n+1)$-симплекса $\Delta$ из $Q$ пусть $\psi_{\Delta}$ обозначает стинродовский цикл $\left.\left.\varphi\right|_{\Delta} \cup h_{k}^{(n+1)}\right|_{R_{[0, \infty)}}: \Delta \cup R_{[0, \infty)} \rightarrow P_{[0, \infty)}$, где $R_{i}=\left(q_{0}^{i}\right)^{-1}(\varphi(\partial \Delta))$ образуют фрактализующую обратную последовательность $\cdots \rightarrow R_{1} \rightarrow R_{0}$. Тогда $[\psi]=\left[\bigsqcup_{\Delta<Q} \psi_{\Delta}\right]$ уже на уровне локально конечных симплициальных цепей в $P_{[0, \infty)}$.

Так как $X$ компактен, он может быть покрыт конечным набором множеств $U_{1}, \ldots, U_{r}$ диаметров, не превышающих $2 \varepsilon$, так что каждое $\tilde{r}_{k}^{(n)}\left(\left(q_{0}^{\infty}\right)^{-1}(\partial \Delta)\right)$ содержится в $U_{i}$ для некоторого $i=i(\Delta)$. Для каждого $i$ рассмотрим стинродовский цикл $\psi_{i}:=\bigsqcup_{i=i(\Delta)} \psi_{\Delta}$. Имеем $[\varphi]=\left[\psi_{1}\right]+\cdots+\left[\psi_{r}\right]$ уже на уровне локально конечных цепей. С другой стороны, каждое $\left[\psi_{i}\right]$ лежит в образе $H_{n}\left(U_{i}\right)$ и, следовательно, тривиально по условию. Это доказывает, что композиция $H_{n}(X) \stackrel{\check{\tau}}{\longrightarrow} \check{H}_{n}(X) \stackrel{p_{k}^{\infty}}{\longrightarrow} H_{n}\left(P_{k}\right)$ инъективна для некоторого $k$, откуда следует искомое утверждение.

(f), (h). Доказываются аналогично соответствующим частям теоремы 6.1.

Зафиксируем один побочный продукт предыдущего обсуждения.

Теорема 6.10'. Если $n>0$, то следствие 6.10 справедливо при более слабом условии $\mathrm{HLC}_{n-1}$.

ДокАЗАТЕльство. Доказательство аналогично доказательству теоремы 6.5 , но проще, поскольку нет нужды беспокоиться о базисных точках и предложение 6.4 может быть заменено построением из доказательства теоремы 6.8 (b).

Теорема 6.11. Пусть $X-$ компакт, $n \geqslant 0$.

(а) Следующие утверждения равносильны:

(i) $X$ удовлетворяет $\mathrm{LC}_{n}$;

(ii) для произвольных замкнутых $Y, Z \subset X$ mаких, что $Y \subset$ Int $Z$, включение $Y \hookrightarrow Z$ пропускается через компакт $W$, удовлетворяющий $\mathrm{LC}_{n}$;

(iii) для произвольных замкнутых $Y, Z \subset X$ mаких, что $Y \subset \operatorname{Int} Z$, образ $\operatorname{im}\left[\pi_{i}(Y) \rightarrow \pi_{i}(Z)\right]$ счетен для всех $i \leqslant n$.

(b) Следующие утверждения равносилъны:

(i) $X$ удовлетворяет $\mathrm{HLC}_{n}$;

(ii) для произвольных замкнутых $Y, Z \subset X$ mаких, что $Y \subset \operatorname{Int} Z$, $Y$ содержится в компакте $W$, удовлетворяющем $\mathrm{LC}_{n}$ и таком, что включение $Y \hookrightarrow Z$ пропускается через включение $Y$ в компакт $\widehat{W}$ такой, что $\widehat{W} \backslash Y-$ фрактализация $W \backslash Y$

(iii) для произвольных замкнутых $Y, Z \subset X$ mаких, ито $Y \subset \operatorname{Int} Z$, групnа $\operatorname{im}\left[H_{i}(Y) \rightarrow H_{i}(Z)\right]$ конечно порождена для всех $i \leqslant n$. 
Импликация (ii) $\Rightarrow$ (iii) в пункте (b) принадлежит Борелю и Муру [16], а обратная импликация - Дыдаку [98]. Раньше всего был доказан когомологический аналог для (i) $\Leftrightarrow$ (iii), принадлежащий Уайлдеру (см. [12]).

ДоказАтельство. (а) Предположим (i). Пусть $Y$ и $Z$ - такие, как в (ii), и предположим, что $(X, Y)=\underset{\lim }{\lim }\left(P_{i}, Q_{i}\right)$. В силу конструкции Ферри (см. доказательство теоремы 6.1$), P_{[k, \infty)}^{(\overleftarrow{n+1})} \cup X$ ретрагируется на $X$ для некоторого $k$. Выберем $l>k$ такое, что эта ретракция переводит $W:=Q_{[l, \infty)}^{(n+1)} \cup Y$ в $Z$. Очевидно, $W$ удовлетворяет $\stackrel{\stackrel{\Delta}{C}}{n}_{n}$.

Предположим (ii). По пунктам (h) и (c) теоремы $6.1, \pi_{i}(W)$ дискретна для всех $i \leqslant n$. Значит, по лемме $3.4(\mathrm{~b})$, каждая $\pi_{i}(W)$ счетна, что влечет (iii).

Предположим (iii). Пусть $\cdots \subset N_{2} \subset N_{1}$ - фундаментальная последовательность замкнутых окрестностей произвольно заданной точки $x \in X$. Тогда по теореме $3.1(\mathrm{c}) \stackrel{\lim }{\longleftarrow} \pi_{k}\left(N_{i}\right)=0=\lim ^{1} \pi_{k}\left(N_{i}\right)$ для всех $k$. Если $G_{i}=$

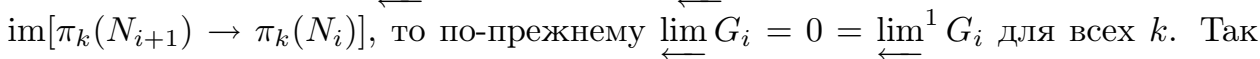
как $G_{i}$ счетны для всех $k \leqslant n$, то по леммам 3.3 и 3.4 (а) для каждого $i$ существует $j>i$ такое, что $G_{j} \rightarrow G_{i}$ тривиально для всех $k \leqslant n$. Это влечет (i).

(b) Импликация (iii) $\Rightarrow$ (i) доказывается аналогично соответствующей импликации в пункте (a). Импликация (i) $\Rightarrow$ (ii) доказывается аналогично соответствующей импликации в пункте (a), с использованием фрактализованной конструкции Ферри.

Предположим (ii). Аналогично доказательству импликации (ii) $\Rightarrow$ (iii) в (a), получаем, что $H_{i}(W)$ отображается инъективно в конечнопорожденную абелеву группу. Следовательно, она конечно порождена, и утверждение следует из коммутативности диаграммы

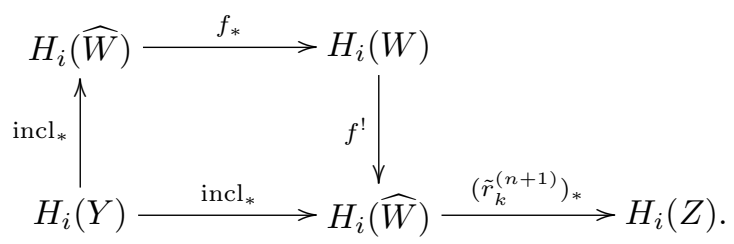

Теорема 6.11 доказана.

Говорят, что обратная последовательность групп $G_{i}$ nочтu ("nearly") удовлетворяет условию Миттаг-Леффлера, если для каждого $i$ существует $j>i$ такое, что для каждого $k>j$ образ $G_{j} \rightarrow G_{i}$ содержится в нормальном замыкании образа $G_{k} \rightarrow G_{i}$. Если $X$ - предел обратной последовательности компактных связных полиэдров $P_{i}$, то свойство обратной последовательности $\pi_{1}\left(P_{i}\right)$ почти удовлетворять условию Миттаг-Леффлера, очевидно, не зависит от выбора базисной точки $X$ и является инвариантом отношения эквивалентности из предложения 2.6 (iii). Тогда можно назвать $X$ nочти связным по Cmuнродy, если это свойство удовлетворено. Таким образом, свойство быть почти связным по Стинроду является (непунктированным) шейповым инвариантом. Оно было введено Макмилланом [99] под другим названием; в [99] доказано, что непрерывный образ почти связного по Стинроду компакта почти связен 
по Стинроду. Очевидны (см. теоремы 3.1 (b), 4.1 (iii) и лемму 3.3) следующие импликации:

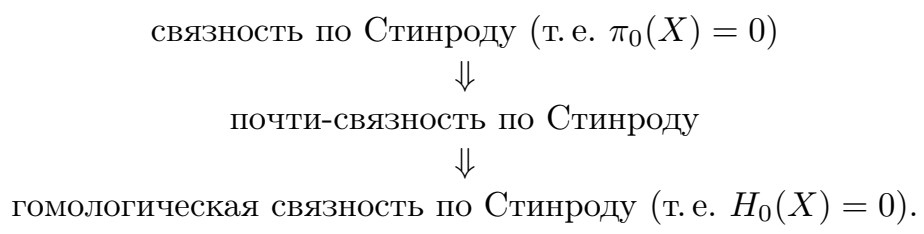

Теорема 6.12. Пусть $X$ - предел обратной последовательности компактных связных полиэдров $P_{i}$. Рассмотрим одноточечную компактификацию $P_{[0, \infty]} / X$ полиэдра $P_{[0, \infty)}$.

(а) (Шриханде [100]) $P_{[0, \infty]} / X$ удовлетворяет $\mathrm{LC}_{1}$ тогда и только тогда, когда $X$ почти связен по Стинроду.

(b) (Дъдак [98]) $P_{[0, \infty]} / X$ удовлетворяет $\mathrm{HLC}_{n}$ тогда и только тогда, когда $H_{i}(X)$ дискретна для всех $i<n$.

Следует отметить, что по теореме 6.1 компакт $P_{[0, \infty]} / X$ удовлетворяет $\mathrm{LC}_{n}$, если и только если он удовлетворяет $\mathrm{LC}_{1}$ и $\mathrm{HLC}_{n}$ одновременно.

ДокАзАтельство (b). Аналогично доказательству теоремы 3.12 устанавливается, что $H_{i}(X)$ дискретна при $i<n$, если и только если для каждого $i$ найдется $j>i$ такое, что $H_{k}\left(P_{[j, \infty]}, X\right)$ тривиально отображается в $H_{k}\left(P_{[i, \infty]}, X\right)$ для каждого $k<n$. По аксиоме вырезания для отображений из теоремы 4.1 (i) последнее эквивалентно выполнению условия $\mathrm{HLC}_{n}$ для $P_{[0, \infty]} / X$ в компактифицирующей точке $X / X$. Теорема 6.12 доказана.

Доказательство части (а) проводится параллельно доказательству теоремы 3.12 ; при необходимости читатель легко восстановит подробности.

\section{§ 7. Теория накрытий}

ТеОРема 7.1. Пусть $X-$ связный локально связный компакт, и пусть $d \in\{1,2, \ldots, \infty\}$. Тогда отображение монодромии доставляет биекцию междy d-кратными накрытиями над $X$ (с точностью до послойного гомеоморфизма) и представлениями топологической группь
(a) $\stackrel{\Delta}{\pi}_{1}(X)$;
(b) $\check{\pi}_{1}(X)\left(\right.$ или $\left.\pi_{1}(X)\right)$

в дискретно топологизированную симметрическую группу $S_{d}$ (с точностью до внутреннего автоморфизма $\left.S_{d}\right)$. Связные накрывающие пространства соответствуют транзитивным представлениям.

Если $X$ полулокально-односвязен, то $\stackrel{\Delta}{\pi}_{1}(X)$ и $\check{\pi}_{1}(X)$ совпадают и являются дискретными по пунктам (d), (f), (h) теоремы 6.1, поэтому обычная теория накрытий - частный случай каждого из утверждений (a) и (b) теоремы 7.1.

Фокс упоминает теорему 7.1 (a) [28; с. 48] со следующим комментарием: "Я доказал это [утверждение] на лекциях в университете Мехико летом 1951 г. С тех пор оно было независимо обнаружено другими и встречается, например, 
в [учебнике Спеньера, [14]], с. 82 [с. 108-109 русского перевода]". Относительно "других" см., в частности, [59].

Используя теорему 6.1 (e), можно вывести утверждение 7.1 (b) непосредственно из 7.1 (a).

ДоКАЗАТЕЛЬСТво ЧАСТи (b). Из следствия 2.5.3, леммы 2.5.11 и теоремы 2.5.13 работы [14] следует, что классы послойного гомеоморфизма связных $d$-кратных накрытий $p: \widetilde{X} \rightarrow X$ находятся в биективном соответствии с классами сопряженности подгрупп индекса $d$ сингулярной фундаментальной группы $\stackrel{\Delta}{\pi}_{1}(X)$, содержащими прообраз некоторой окрестности 1 в $\check{\pi}_{1}(X)$ при композиции $\stackrel{\Delta}{1}_{1}(X) \stackrel{\stackrel{\Delta}{\tau}}{\rightarrow} \pi_{1}(X) \stackrel{\check{\tau}}{\rightarrow} \check{\pi}_{1}(X)$. Так как $\check{\pi}_{1}(X)$ нульмерна, то базис окрестностей 1 задается всеми открыто-замкнутыми множествами. Следовательно, рассматриваемые подгруппы - в точности те же, что содержат ядро композиции $\check{\tau} \stackrel{\Delta}{\tau}$ с некоторым непрерывным представлением $\check{\pi}_{1}(X)$ в дискретную группу. С другой стороны, по теореме 6.1 (е), образ $\check{\tau} \stackrel{\Delta}{\tau}$ плотен в $\check{\pi}_{1}(X)$. Значит, классы послойного гомеоморфизма связных $d$-кратных накрывающих $X$ находятся в биективном соответствии с классами сопряженности подгрупп индекca $d$ группы $\check{\pi}_{1}(X)$, содержащими ядро какого-либо непрерывного представления в какую-либо дискретную группу. Если $H$ - подобная подгруппа индекса $d$, то пересечение $K$ всех сопряженных ей подгрупп содержит ядро гомоморфизма в дискретную группу, поэтому $\check{\pi}_{1}(X) / K$ дискретна. Действие $\check{\pi}_{1}(X) / K$ на правых смежных классах своей подгруппы $H / K$ индекса $d$ доставляет транзитивное представление $\check{\pi}_{1}(X) / K \rightarrow S_{d}$. Этим доказан транзитивный случай пункта (b), из которого уже следует и общий, если учесть, что локально-связный компакт имеет открытые компоненты связности.

Чтобы обобщить теорему 7.1 на пространства, не являющиеся локальносвязными, Фокс "подправил" понятие накрытия [28], [101]. Небольшая неточность в теории Фокса была, в свою очередь, исправлена в [102]. См. также [103] и ссылки там. Напомним, что накрытие - это отображение $p: \widetilde{X} \rightarrow X$ такое, что существует покрытие $\left\{U_{\alpha}\right\}$ пространства $X$, удовлетворяющее следующему условию:

(i) каждое $p^{-1}\left(U_{\alpha}\right)$ есть $\bigsqcup_{\lambda} U_{\alpha}^{\lambda}$, причем каждое $\left.p\right|_{U_{\alpha}^{\lambda}}-$ гомеоморфизм.

В этом случае мы будем говорить, что покрытие $\left\{U_{\alpha}^{\lambda}\right\}$ пространства $\widetilde{X}$ лежит над $\left\{U_{\alpha}\right\}$. Cтруктура наложения на $p$ - это покрытие $\left\{U_{\alpha}^{\lambda}\right\}$ пространства $\tilde{X}$, лежащее над некоторым покрытием пространства $X$ и дополнительно удовлетворяющее условию

(ii) если $U_{\alpha}^{\lambda} \cap U_{\beta}^{\mu}$ и $U_{\alpha}^{\lambda} \cap U_{\beta}^{\nu}$ оба непусты, то $\mu=\nu$.

Две структуры наложения $\left\{U_{\alpha}^{\lambda}\right\}$ и $\left\{V_{\alpha}^{\lambda}\right\}$ на $p$ эквивалентны, если существует структура наложения $\left\{W_{\alpha}^{\lambda}\right\}$ на $p$, в которую каждая из них вписывается (как покрытие пространства $\widetilde{X}$ ). Наложение - это класс эквивалентности структур наложения на накрытии. Изоморфизм наложений $\left[p: \widetilde{X} \rightarrow X ;\left\{U_{\alpha}^{\lambda}\right\}\right]$ и $\left[p^{\prime}: \widetilde{X}^{\prime} \rightarrow X ;\left\{V_{\beta}^{\lambda}\right\}\right]$ - это послойный гомеоморфизм $h: \widetilde{X} \rightarrow \widetilde{X}^{\prime}$ (т. е. $p h=p^{\prime}$ ) такой, что $\left\{h\left(U_{\alpha}^{\lambda}\right)\right\}$ и $\left\{V_{\beta}^{\lambda}\right\}$ эквивалентны как структуры наложения на $p^{\prime}$.

ПРЕДЛОЖЕНИЕ 7.2 [101], [102]. Если Х локалъно-связно, то каждое накрьтие над $X$ допускает единственную структуру наложения. 
Доказательство Мура [102] помогает уяснить смысл вышеприведенных определений, поэтому мы его воспроизводим.

ДоказАтельство (см. [102]). Если $\left\{V_{\beta}\right\}$ - покрытие $X$ связными множествами, ясно, что любые две структуры наложения на заданном накрывающем отображении $p$, лежащие над $\left\{V_{\beta}\right\}$, будут совпадать с точностью до перенумерации. Для построения такого $\left\{V_{\beta}\right\}$ заметим, что каждая точка $x \in X$ имеет связную окрестность, так как по условию компонента связности $X$, содержащая $x$, содержит и окрестность $x$. Следовательно, в любое покрытие $\left\{U_{\alpha}\right\}$ пространства $X$ можно вписать покрытие $\left\{V_{\beta}\right\}$, в котором каждое $V_{\beta}$ связно. Если $\left\{U_{\alpha}^{\lambda}\right\}$ - структура наложения, лежащая над $\left\{U_{\alpha}\right\}$, то условие (ii) гарантирует, что в нее можно вписать $\left\{V_{\beta}^{\lambda}\right\}$, лежащее над $\left\{V_{\beta}\right\}$. Таким образом, любые две структуры наложения на заданном накрытии $X$ эквивалентны.

С другой стороны, предположим, что $\left\{U_{\alpha}^{\lambda}\right\}$ лежит над $\left\{U_{\alpha}\right\}$, a $\left\{V_{\beta}\right\}$ вписано в $\left\{U_{\alpha}\right\}$. Если каждое $V_{\beta}$ связно, то в $\left\{U_{\alpha}^{\lambda}\right\}$ можно вписать $\left\{V_{\beta}^{\lambda}\right\}$, лежащее над $\left\{V_{\beta}\right\}$. Если, дополнительно, $V_{\beta} \cap V_{\gamma} \neq \varnothing$ влечет $V_{\beta} \cup V_{\gamma} \subset U_{\alpha}$ для некоторого $\alpha$, то $\left\{V_{\beta}^{\lambda}\right\}$ будет структурой наложения. Для построения такого $\left\{V_{\beta}\right\}$ рассмотрим какое-нибудь покрытие $\left\{W_{\gamma}\right\}$ пространства $X$ множествами диаметра не больше $\lambda / 2$, где $\lambda$ - число Лебега покрытия $\left\{U_{\alpha}\right\}$ (т. е. всякое подмножество $X$ диаметром не больше $\lambda$ содержится хотя бы в одном $\left.U_{\alpha}\right)$. Тогда $W_{\beta} \cap W_{\gamma}=\varnothing$ влечет $W_{\beta} \cup W_{\gamma} \subset U_{\alpha}$ для некоторого $\alpha$. Наконец, $\left\{V_{\beta}\right\}$ определяется как произвольное покрытие со связными элементами, вписанное в $\left\{W_{\gamma}\right\}$. Предложение 7.2 доказано.

Существует накрытие над $\mathbb{N} \times I \cup[0, \infty] \times \partial I$, не допускающее структуры наложения [101; рис. 1, 2 и с. 78]. Это особенно легко увидеть, используя лемму 7.3 (а), ср. [102; пример 1].

Если $X$ - одномерная гавайская улитка из примера 5.7 , т. е. тор вложения из $\{0\} \cup\left\{2^{-n} \mid n \in \mathbb{N}\right\}$ в себя, определенного формулой $x \mapsto x / 2$, то ее универсальное накрытие допускает несчетное количество попарно неэквивалентных структур наложения. Однако все они изоморфны друг другу посредством накрывающих преобразований, которые не являются авто-изоморфизмами никакой структуры наложения (ср. [28; пример 3]). Используя это замечание и лемму $7.3(\mathrm{~b})$, легко построить накрытие над $S^{1} \vee X$ (где базисная точка пространства $X$ не лежит на предельной окружности), допускающее несчетное количество попарно неизоморфных структур наложения [102; пример 3]. (Заметим, что пример 2 в [102] ошибочен, что видно из леммы 7.3 (а), или следствия 7.5, или же теоремы 7.6.)

Следующая лемма доставляет упрощение [28; теорема о продолжении 5.2], [102; теорема 1.5].

Лемма 7.3. (а) Накрытие над компактом допускает структуру наложения, если и только если оно индуцировано из накрытия над полиэдром.

(b) Наложения $f^{*}(p), g^{*}(q)$ над компактом $X$, индуцированные из накрьтий $p, q$ над полиэдрами $P, Q$, изоморфны, если и только если $f$ и $g$ разлагаются с точностъю до гомотопии как $f: X \stackrel{h}{\rightarrow} R \stackrel{f_{1}}{\longrightarrow} P$ u $g: X \stackrel{h}{\longrightarrow} R \stackrel{g_{1}}{\longrightarrow} Q$, где $R$ - полиэдр и $f_{1}^{*}(p)=g_{2}^{*}(q)$. 
Доказательство. (а) Ясно, что всякое накрытие, индуцированное из наложения, приходит вместе с индуцированной структурой наложения. Обратно, предположим, что задано накрытие $p$ над компактом $X$ со структурой наложения $\left\{U_{\alpha}^{\beta}\right\}$, лежащей над $\left\{U_{\alpha}\right\}$. Если $U_{\alpha}^{\lambda} \cap U_{\beta_{1}}^{\mu_{1}} \cap \cdots \cap U_{\beta_{r}}^{\mu_{r}}$ и $U_{\alpha}^{\lambda} \cap U_{\beta_{1}}^{\nu_{1}} \cap \cdots \cap U_{\beta_{r}}^{\nu_{r}}$ оба непусты, то каждое $\mu_{i}$ равно $\nu_{i}$. Следовательно, очевидное отображение $q$ нерва покрытия $\left\{U_{\alpha}^{\lambda}\right\}$ на нерв $N$ покрытия $\left\{U_{\alpha}\right\}$ является накрытием. Таким образом, $p$ индуцировано из накрытия $q$ посредством проекции $X \rightarrow N$.

(b) Пусть $\left\{U_{\alpha}\right\}$ и $\left\{V_{\beta}\right\}$ - покрытия $P$ и $Q$ открытыми звездами вершин некоторых триангуляций. Каждое $U_{\alpha} \cap U_{\gamma}$ и каждое $V_{\beta} \cap V_{\delta}$ связны, поэтому $p$ и $q$ допускают структуры наложения, лежащие над $\left\{U_{\alpha}\right\}$ и $\left\{V_{\beta}\right\}$. Пусть $\left\{W_{\gamma}^{\lambda}\right\}-$ структура наложения на накрытии $f^{*}(p)=g^{*}(q)$, вписанная сразу в обе индуцированные структуры наложения. Если $\left\{W_{\gamma}^{\lambda}\right\}$ лежит над $\left\{W_{\gamma}\right\}$, то $f$ и $g$ пропускаются с точностью до гомотопии через нерв $N$ накрытия $\left\{W_{\gamma}\right\}$ и отображения $N \rightarrow P$ и $N \rightarrow Q$ индуцируют одно и то же накрытие из $p$ и $q$.

Лемма 7.3 доказана.

Следующий результат представляет собой упрощение формулировки теоремы Фокса о классификации наложений [28].

Теорема 7.4. Пусть $X-$ связный компакт $u d \in\{1,2, \ldots, \infty\}$. Существует естественная биекиия между $\left[X, B S_{d}\right]$ и множеством классов изоморфизма d-кратных наложений над $X$.

Заметим, что если $X$ - полиэдр, это всего лишь переформулировка полиэдрального случая теоремы 7.1.

ДокАЗАТЕльство. По лемме 7.3 (а) каждое наложение над $X$ индуцировано из накрытия над некоторым полиэдром $P$ посредством отображения $f: X \rightarrow P$. Это доставляет отображение $X \rightarrow P \rightarrow B S_{d}$, гомотопический класс которого корректно определен по лемме $7.3(\mathrm{~b})$. Обратно, если $X$ - предел обратной последовательности полиэдров $P_{i}$, то по лемме 2.1 (а) каждое отображение $f: X \rightarrow B S_{d}$ продолжается на $P_{[i, \infty]}$ для некоторого $i$. Значит, оно пропускается с точностью до гомотопии через $X \rightarrow P_{i}$ и тем самым порождает наложение. Если $f$ гомотопно отображению $g: X \rightarrow B S_{d}$, которое продолжается на $P_{[j, \infty]}$, то по лемме 2.1 (b) два имеющихся продолжения гомотопны на $P_{[k, \infty]}$ для некоторого $k$ и, следовательно, определяют одно и то же наложение. Утверждение теоремы теперь следует из предложения 2.3 (а). Теорема 7.4 доказана.

По предложению $2.3(\mathrm{~b}),\left[X, B S_{d}\right]=\underline{\lim }\left[P_{i}, B S_{d}\right]$, где компакт $X$ - обратный предел полиэдров $P_{i}$. С другой стороны, $\overrightarrow{\lim }\left[P_{i}, B S_{d}\right]$ может быть отождествлен с множеством орбит действия $S_{d}$ на $\lim \overrightarrow{\operatorname{Hom}}\left(\pi_{1}\left(P_{i}\right), S_{d}\right)$, порожденного внутренними автоморфизмами $S_{d}$. Известно (и несложно видеть), что обратные последовательности групп, удовлетворяющие условию Миттаг-Леффлера, сводятся к своим топологическим обратным пределам [104; §2]. Отсюда получаем следующее обобщение теоремы 7.1 .

СлеДСтвиЕ 7.5 [105]. Пусть $X$ - связный по Стинроду компакт $u d \in$ $\{1,2, \ldots, \infty\}$. Отображение монодромии доставляет биекцию между классами изоморфизма $d$-кратных наложений над $X$ и классами сопряженности 
представлений топологической группь $\check{\pi}_{1}(X)\left(\right.$ или $\left.\pi_{1}(X)\right)$ в дискретно топологизированную симметрическую группу $S_{d}$.

Следствие 7.5 не выполнено для произвольных компактов: существует свободное действие группы $\mathbb{Z} / 3$ на 2-адическом соленоиде $\Sigma_{2}$, с пространством орбит, снова гомеоморфным $\Sigma_{2}$; при этом $\check{\pi}_{1}\left(\Sigma_{2}\right)=0$.

Отметим, что добавление о транзитивных представлениях, входящее в теорему 7.1, опущено в следствии 7.5: и вправду, оно ложно в такой общности, как видно из рассмотрения одномерной гавайской улитки (см. [28; пример 3], ср. [101]). Можно показать, однако, что оно будет выполнено, если "связность" понимать в смысле равномерных, а не топологических пространств, как в следующей теореме.

ТеОрема 7.6. Пусть X - компакт. Существует естественная биекиия между множеством классов эквивалентности структур наложения на накрытии $p: \widetilde{X} \rightarrow X$ и множеством равномерностей на $\widetilde{X}$, согласованных с $p$.

Мы говорим, что равномерность (т. е. равномерная структура) на $\widetilde{X}$ согласована с накрытием $p: \widetilde{X} \rightarrow X$, где $X-$ компакт, если каждая $x \in X$ имеет замкнутую окрестность $U$ такую, что

(iii) $p^{-1}(U)=\bigsqcup_{\lambda} U^{\lambda}$ как равномерные пространства, причем каждое $\left.p\right|_{U^{\lambda}}-$ гомеоморфизм.

Таким образом, условия Фокса (i) и (ii) могут быть заменены единым условием (iii). Условие (iii) можно сформулировать следующим равносильным образом:

$\left(\right.$ iii' $\left.^{\prime}\right) p^{-1}(U)$ равномерно гомеоморфно $U \times F$, где $F$ дискретно, посредством гомеоморфизма $h$ такого, что $p h^{-1}: U \times F \rightarrow U-$ проекция.

Подчеркнем, что $F$ предполагается дискретным как равномерное пространство, что является более сильным условием, чем дискретность индуцированной топологии. Например, $\{\ln 1, \ln 2, \ldots\} \subset \mathbb{R}$ дискретно как топологическое подпространство $\mathbb{R}$, но не дискретно как равномерное подпространство $\mathbb{R}$, поскольку $\ln (n+1)-\ln n \rightarrow 0$ при $n \rightarrow \infty$. За информацией о равномерных пространствах мы отсылаем читателя к книге Исбелла [11].

Приведенное выше определение равносильно введенному Я. М. Джеймсом понятию накрытия в категории равномерных пространств (см. [106], где определение Джеймса немного подправлено). Теорему 7.6 можно рассматривать как усовершенствование результата работы [102], но автор пришел к ней, анализируя пример 5.8 .

ДокАЗАТЕЛЬСтво. Предположим, что $X$ - предел обратной последовательности компактных полиэдров $P_{i}$. Мы можем предполагать, что они триангулированы так, что каждое связующее отображение симплициально как отображение $P_{i+1} \rightarrow P_{i}^{\prime}$ в барицентрическое измельчение $P_{i}$. По лемме 7.3 (a) (см. также доказательство теоремы 7.4) мы можем предполагать, что заданное наложение $p$ индуцировано из накрытия $q_{k}$ над некоторым $P_{k}$. Для каждого $j>k$ пусть $q_{j}$ - накрытие над $P_{j}$, индуцированное из $q_{k}$, и пусть $E_{j}$ - его тотальное пространство. Тогда $\widetilde{X}-$ предел обратной последовательности равномерно непрерывных отображений $E_{j+1} \rightarrow E_{j}$, где каждое $E_{j}$ снабжено стандартной 
метрикой, которая соответствует триангуляции $E_{j}$, в которой $q_{j}$ симплициально. Этим определена равномерность на $X$, и по лемме 7.3 (а) (см. также доказательство теоремы 7.4) она не зависит от выбора $q_{k}$.

Для заданной равномерности на $\tilde{X}$, согласованной с $p$, пусть $\left\{U_{\alpha}\right\}-$ покрытие $X$, где каждое $U_{\alpha}$ удовлетворяет условию (iii'). Не теряя общности, мы можем предполагать, что это покрытие конечно. Зафиксируем некоторую метрику на $X$, и пусть $d$ - диаметр $X$. Рассмотрим метрику на $F$, в которой расстояние между любыми двумя точками равно $2 d$. Представим равномерность на $\widetilde{X}$ с помощью некоторой метрики. Пусть $\delta>0$ таково, что $\delta$-близкие точки пространства $\widetilde{X}$ остаются $d$-близкими под действием каждого $h_{\alpha}: p^{-1}\left(U_{\alpha}\right) \rightarrow U_{\alpha} \times F$, и пусть $\varepsilon>0$ таково, что $\varepsilon$-близкие точки каждого $U_{\alpha} \times F$ остаются $\delta$-близкими под действием каждого $h_{\alpha}^{-1}$. Впишем в $\left\{U_{\alpha}\right\}$ покрытие $\left\{V_{\beta}\right\}$ такое, что каждое $\left\{V_{\beta}\right\}$ имеет диаметр не больше $\varepsilon$. Если $V_{\beta}$ лежит одновременно в $U_{\alpha}$ и в $U_{\gamma}$, то ограничения $V_{\beta} \times F \stackrel{h_{\alpha}^{-1}}{\longrightarrow} p^{-1}\left(V_{\beta}\right) \stackrel{h_{\gamma}}{\longrightarrow} V_{\beta} \times F$ обязаны иметь вид $\operatorname{id}_{V_{\beta}} \times \sigma$ для некоторой перестановки $\sigma \in S_{F}$. Значит, если $V_{\beta}^{\lambda}=h_{\alpha}\left(V_{\beta} \times\{\lambda\}\right)$, то покрытие $\left\{V_{\beta}^{\lambda}\right\}$ пространства $\widetilde{X}$ корректно определено. Если $\left\{V_{\beta}\right\}$ выбрано так, что, кроме того, $V_{\beta} \cap V_{\gamma} \neq \varnothing$ влечет $V_{\beta} \cup V_{\gamma} \subset U_{\alpha}$ для некоторого $\alpha$ (см. доказательство предложения 7.2), то $\left\{V_{\beta}^{\lambda}\right\}-$ структура наложения. Ясно, что любые две структуры наложения, определенные таким образом, эквивалентны.

СЛЕДСТВИЕ 7.7. Послойный гомеоморфизм налегающих пространств является изоморфизмом тогда и только тогда, когда он является равномерным гомеоморфизмом соответствующих равномерностей.

СЛЕДСТВИЕ 7.8 [101], [102]. Всякое конечнократное накрытие над компактом $X$ допускает единственную структуру наложения.

Оставшаяся часть настоящего параграфа посвящена наброску того, каким образом налегающие пространства компактов могут быть включены в стинродовскую теорию гомотопий. Эти построения пригодятся нам кое-где в следующем параграфе (в первую очередь - в доказательстве теоремы 8.9) и, в свою очередь, предполагают знакомство читателя с метризуемыми равномерными пространствами (см. [11]).

Равномерный компактифицированный телескоп. Если $X$ - метризуемое равномерное пространство, то конус $C X:=X \times I / X \times\{0\}$ наделяется фактор-равномерностью, которая метризуема (см. [107]) в отличие от фактортопологии. Цилиндр $M C(f)$ равномерно непрерывного отображения $f: X \rightarrow Y$ между метризуемыми равномерными пространствами - это образ $\Gamma_{f} \times I \subset$ $X \times Y \times I$ в метризуемом равномерном пространстве $C X \times Y$. Если $X$ полно, то полон и $C X$, и если дополнительно $Y$ полно, то полон и $M C(f)$.

Далее, если $A$ - замкнутое подмножество метризуемого равномерного пространства $X$, то равномерное факторпространство $X / A$ метризуемо (см. [107]). Отсюда несложно получить, с использованием стандартной техники равномерных абсолютных ретрактов (см. [11], где они называются “injective spaces"), что всякая ограниченная метрика на $A$, индуцирующая его равномерность подпространства, продолжается до ограниченной метрики на $X$, индуцирующей его 
исходную равномерность. (Аналогичная лемма о продолжении известна для псевдометрик [11; III.16].)

Склейка $X \cup_{h} Y$ метризуемых равномерных пространств по равномерному гомеоморфизму $h$ между замкнутым подмножеством $X$ и замкнутым подмножеством $Y$ наделяется факторравномерностью $(X \sqcup Y) / h$, которая метризуема (см. [108; лемма I.5.24]) и корректно определена в силу вышеупомянутой леммы о продолжении метрик. Если $X$ и $Y$ полны, полна и $X \cup_{h} Y$.

Рассмотрим обратную последовательность $X=\left(\cdots \stackrel{f_{1}}{\longrightarrow} X_{1} \stackrel{f_{0}}{\longrightarrow} X_{0}\right)$ равномерно непрерывных отображений между метризуемыми равномерными пространствами. Пусть $X_{[0, \infty]}$ - обратный предел конечных телескопов $X_{[0, n]}=$ $M C\left(f_{0}\right) \cup_{X_{1}} M C\left(f_{1}\right) \cup_{X_{2}} \cdots \cup_{X_{n-1}} M C\left(f_{n-1}\right)$ и очевидных ретракций $[0, n+1] \rightarrow$ $X_{[0, n]}$. Если задано $J \subset[0, \infty]$, обозначим через $X_{J}$ прообраз $J$ при проекции $X_{[0, \infty]} \rightarrow[0, \infty]$. Согласно вышесказанному, если каждое $X_{i}$ полно, то полон и $X_{[0, \infty]}$. В этом случае он является пополнением бесконечного телескопа $X_{[0, \infty)}$.

Сходимость и условие Коши для обратных последовательностей. В обозначениях предыдущего абзаца, будем называть обратную последовательность $X$ сходящейся, если каждая равномерная окрестность $\lim _{\longleftarrow} X$ в $X_{\mathbb{N} \cup \infty}$ (или, что равносильно, в $\left.X_{[0, \infty]}\right)$ содержит все, кроме конечного числа, $X_{i}$. Скажем, что $X$ является обратной последовательностью Коши, если для каждого $\varepsilon>0$ существует $k$ такое, что для каждого $j>k \varepsilon$-окрестность $X_{j}$ в $X_{\mathbb{N}}$ (или, что равносильно, в $\left.X_{[0, \infty)}\right)$ содержит $X_{k}$. Ясно, что введенные понятия корректно определены в категории равномерных пространств. Вот пример обратной последовательности Коши, которая расходится: $\cdots \subset(0,1 / 4] \subset(0,1 / 2] \subset(0,1]$. Обратная последовательность $\cdots \subset[2, \infty) \subset[1, \infty) \subset[0, \infty)$ не является последовательностью Коши. ${ }^{20}$

Имеющая место прямая аналогия с определениями сходимости и условия Коши для последовательности точек в метрическом пространстве может в действительности быть формализована. Если $M$ - метрическое пространство, то пространство $2^{M}$ всех непустых замкнутых подмножеств $M$ наделяется метрикой Хаусдорфа $d_{H}\left(A_{1}, A_{2}\right)=\min \left(\max \left(d_{1}, d_{2}\right), 1\right)$, где $d_{i}=\sup \left\{d\left(a, A_{i}\right) \mid a \in A_{j}\right.$, $j \neq i\}$. Если $Y$ - метризуемое равномерное пространство, то индуцированная равномерная структура на $2^{Y}$ корректно определена, и если $Y$ полон или компактен, то же выполнено и для $2^{Y}$ [11; II.48, II.49]. Мы получаем, что обратная последовательность $X$ сходится (удовлетворяет условию Коши), если и только если последовательность замкнутых подмножеств $X_{i}$ пространства $X_{\mathbb{N} \cup \infty}$ сходится (удовлетворяет условию Коши) как последовательность точек пространства $2^{X_{\mathbb{N} \cup \infty}}$ или, равносильно, пространства $2^{X_{[0, \infty]}}$. Отсюда следуют утверждения (a) и (b) следующей леммы.

Лемма 7.9. Пусть $X=\left(\cdots \stackrel{f_{1}}{\longrightarrow} X_{1} \stackrel{f_{0}}{\longrightarrow} X_{0}\right)$ - обратная последовательность равномерно непрерывных отображений между метризуемыми равномерными пространствами.

(а) Если каждое $X_{i}$ компактно, то $X$ сходится.

\footnotetext{
20 Читатель, знакомый с "резольвентами" Мардешича, легко убедится, что сходящаяся обратная последовательность равномерно непрерывных отображений между метризуемыми полными равномерными пространствами является резольвентой Мардешича.
} 
(b) Если X сходится, то она удовлетворяет условию Коши; обратное имеет место, когда каждое $X_{i}$ полно.

(с) $X$ сходится, если и только если для каждого $i$ каждая равномерная окрестность $f_{i}^{\infty}\left(\lim _{\longleftarrow} X\right)$ в $X_{i}$ содержит все, кроме конечного числа, $f_{i}^{j}\left(X_{j}\right)$.

(d) $X$ удовлетворяет условию Коши, если и только если для каждого $i$ и каждого $\varepsilon>0$ существует $k$ такое, что для каждого $j>k$-окрестность $f_{i}^{j}\left(X_{j}\right)$ в $X_{i}$ содержит $f_{i}^{k}\left(X_{k}\right)$.

(е) Если каждое $X_{i}$ равномерно дискретно, то $X$ сходится, если и только если она удовлетворяет условию Миттаг-Лефблера.

(f) Если $X$ сходится и каждое $X_{i}$ непусто (соответственно равномерно связно), то $\lim X$ непуст (соответственно равномерно связен).

(g) Если $Y=\left(\cdots \stackrel{f_{1}}{\longrightarrow} X_{1} \stackrel{f_{0}}{\longrightarrow} X_{0}\right)$ - еще одна обратная последовательность равномерно непрерывных отображений между метрическими пространствами, $f_{i}: X_{i} \rightarrow Y_{i}$ - сюргекиии, коммутирующие со связующими отображсениями, и $X$ сходится (удовлетворяет условию Коши), то $Y$ сходится (удовлетворяет условию Коши).

ДокАзАтельство. (а) Выше мы уже вывели (а) из общих фактов, но может представлять интерес и элементарное доказательство. Если $X$ расходится, то существует открытая окрестность $U$ пространства $X$ в $X_{\mathbb{N} \cup \infty}$, дополнение которой имеет непустое пересечение $C_{i}$ с $X_{\{i, i+1, \ldots, \infty\}}$ для каждого $i$. По теореме Кантора компакты $C_{i}$ имеют непустое пересечение. Оно содержится в $\bigcap X_{\{i, i+1, \ldots, \infty\}}=X$ и, в то же время, в дополнении к $U$, что приводит к противоречию.

(c) Покрытие пространства $\lim X_{\{0, \ldots, i\}}$ равномерно, если и только если в него можно вписать прообраз (см. [11]). Тогда окрестность $X_{\infty}$ в $X_{\mathbb{N} \cup\{\infty\}}$ равномерна, если и только если она содержит прообраз $V$ некоторой равномерной окрестности $U$ образа $f_{i}^{\infty}\left(X_{\infty}\right)$ в $X_{\{0, \ldots, i\}}$ для некоторого $i$. Но это $U$ содержит $f_{i}^{j}\left(X_{j}\right)$ (которые лежат в $\left.X_{i} \subset X_{\{0, \ldots, i\}}\right)$ для почти всех $j>i$, если и только если $V$ содержит $X_{j}$ для почти всех $j>i$.

(d)-(g). Часть (d) доказывается аналогично (c). Части (e) и (f) следуют из (c). Часть (g) следует из (c) и (d).

СлеДствиЕ 7.10 (бурбаковская “теорема Миттаг-Леффлера”). Пусть L предел обратной последователъности $\cdots \stackrel{f_{1}}{\longrightarrow} X_{1} \stackrel{f_{0}}{\longrightarrow} X_{0}$ равномерно непреръвных отображений между метризуемыми полными равномерными пространствами. Если каждый $f_{i}\left(X_{i+1}\right)$ плотен в $X_{i}$, то тогда и $f_{0}^{\infty}(L)$ плотен в $X_{0}$.

ДокАЗАТЕЛЬСтво. Из условия следует, что каждый $f_{i}^{j}\left(X_{j}\right)$ плотен в $X_{i}$. Значит, по лемме 7.9 (d) $X$ удовлетворяет условию Коши. Тогда по лемме 7.9 (b) $X$ сходится. Если $U$ - равномерная окрестность $f_{0}^{\infty}(L)$, то по лемме 7.9 (c) она содержит $f_{0}^{j}\left(X_{j}\right)$ для некоторого $j$. Если $U$ замкнута, отсюда следует, что $U=X_{0}$. Поскольку $U$ произвольна, $f_{0}^{\infty}(L)$ плотен в $X_{0}$. Следствие 7.10 доказано.

Известно, что этот результат обобщается на случай обратных спектров, отличных от обратных последовательностей (см. "Общую топологию” Бурбаки), 
и, в другом направлении, на случай непрерывных, но не равномерно непрерывных связующих отображений [109]. Небольшой модификацией рассуждения из [109] мы также получаем следующее утверждение.

СледСтвиЕ 7.11 (теорема Бэра о категории). Пересечение счетного семейства плотных открытых множеств в полном метризуемом равномерном пространстве плотно.

ДокАЗАТЕЛЬство. Не теряя общности, мы можем предполагать, что данные плотные открытые множества $U_{i}$ упорядочены включением: $U_{1} \supset U_{2} \supset \cdots$. Если $d$ - метрика на $U_{0}:=X$, пусть $d_{0}=d$, и определим метрику на $U_{i+1}$ формулой

$$
d_{i+1}(x, y)=d_{i}(x, y)+\left|\frac{1}{d_{i}\left(x, U_{i} \backslash U_{i+1}\right)}-\frac{1}{d_{i}\left(y, U_{i} \backslash U_{i+1}\right)}\right|
$$

Тогда $d_{i+1}$ - полная метрика на $U_{i+1}$ такая, что отображение id: $\left(U_{i+1}, d_{i+1}\right) \rightarrow$ $\left(U_{i+1}, d_{i}\right)$ - равномерно непрерывный гомеоморфизм.

Утверждение следует из теоремы Миттаг-Леффлера, примененной к обратной последовательности $\cdots \rightarrow\left(U_{1}, d_{1}\right) \rightarrow\left(U_{0}, d_{0}\right)$.

Расширение стинродовской гомотопической категории. Мы называем равномерное пространство $X$ конечномерно-аппроксимируемым, если во всякое равномерное покрытие $X$ можно вписать покрытие конечной кратности (т. е. с конечномерным нервом); в [31] такие пространства названы "finitistic". Метризуемая равномерность на конечномерном полиэдре определена в [11]; она является полной. Конечномерно-аппроксимируемые метризуемые полные равномерные пространства - это в точности пределы сходящихся обратных последовательностей конечномерных равномерных полиэдров и равномерно непрерывных связующих отображений [11; V.33]. В частности, они включают все налегающие пространства над компактами.

Пусть $X$ и $Y$ - пределы сходящихся обратных последовательностей $X=$ $\underset{\lim }{\longleftarrow}\left(\cdots \stackrel{p_{1}}{\longrightarrow} P_{1} \stackrel{p_{0}}{\longrightarrow} P_{0}\right)$ и $Y=\underset{\lim }{\longleftarrow}\left(\cdots \stackrel{q_{1}}{\longrightarrow} Q_{1} \stackrel{q_{0}}{\longrightarrow} Q_{0}\right)$ конечномерных равно-

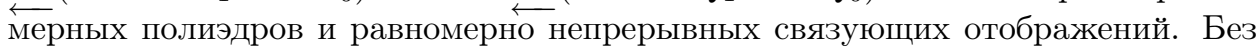
ограничения общности можно считать, что $P_{0}=$ pt и $Q_{0}=p t$. Тогда, аналогично доказательству леммы 2.1 , всякое равномерно непрерывное отображение $f: X \rightarrow Y$ продолжается до равномерно непрерывного отображения $f_{[0, \infty]}: P_{[0, \infty]} \rightarrow Q_{[0, \infty]}$, переводящего $P_{[0, \infty)}$ в $Q_{[0, \infty)}$. Более того, всякие два таких продолжения соединяются равномерно непрерывной (как отображение $\left.P_{[0, \infty]} \times I \rightarrow Q_{[0, \infty]}\right)$ гомотопией неподвижно на $X$, переводящей $P_{[0, \infty)} \times I$ в $Q_{[0, \infty)}$. Напомним, что отображение $F: P_{[0, \infty)} \rightarrow Q_{[0, \infty)}$ мы называем полусобственным, если для любого $k$ существует $l$ такое, что $f^{-1}\left(Q_{[0, k]}\right) \subset P_{[0, l]}$.

Определим стинродовский гомотопический класс $X \rightsquigarrow Y$ как полусобственный гомотопический класс полусобственного отображения $f: P_{[0, \infty)} \rightarrow Q_{[0, \infty)}$. Благодаря полусобственным гомотопическим эквивалентностям $\left(\operatorname{id}_{X}\right)_{[0, \infty)}$ и $\left(\operatorname{id}_{Y}\right)_{[0, \infty)}$, смысл этого определения не зависит от выбора обратных последовательностей $P$ и $Q$. Стинродовские гомотопические группы конечномерноаппроксимируемого метризуемого полного равномерного пространства определяются теперь по аналогии с $\S 3$ (частный случай был рассмотрен во введении). 
Также по аналогии с $\S 4$ определяются стинродовские гомологии и понтрягинские когомологии такого пространства, однако для целей настоящей статьи они не потребуются.

Универсальное обобщенное наложение. Пусть $X$ - стинродовски связный компакт (к общему случаю связного компакта мы еще будем неоднократно возвращаться в следующем параграфе). Если $X$ - предел обратной последовательности компактных полиэдров $P_{i}$, пусть $\widetilde{X}=\longleftarrow \lim \left(\cdots \rightarrow \widetilde{P}_{1} \rightarrow \widetilde{P}_{0}\right)$, где $\widetilde{P}_{i}$ - их универсальные накрывающие. В силу самого их построения они наделены равномерными структурами (унаследованными из полиэдров $P_{i}$ при помощи накрывающих отображений). Поскольку $P_{i}$ сходятся, сходятся и $\widetilde{P}_{i}$. Отсюда вытекает, что стинродовский гомотопический тип конечномерно-аппроксимируемого метризуемого полного равномерного пространства $\widetilde{X}$ не зависит от выбора полиэдров $P_{i}$. С некоторыми усилиями можно показать даже, что от этого выбора не зависит и класс послойного равномерного гомеоморфизма $\widetilde{X}[110]$ (другое доказательство должно появиться в качестве побочного продукта в планируемом продолжении настоящей статьи). По определению, $p: \widetilde{X} \rightarrow X-$ стинродовское расслоение; оно также является серровским расслоением (см. условие (ii) в обсуждении перед формулировкой теоремы 3.15). Каждый слой $p^{-1}(\mathrm{pt})$ равномерно нульмерен, т. е. является обратным пределом (счетных) дискретных равномерных пространств. Очевидно, $\pi_{0}(\widetilde{X})=\mathrm{pt}$ и $\pi_{1}(\widetilde{X})=1$. В случае, когда $\check{\pi}_{1}(X)$ дискретна, $p$ будет наложением, которое можно назвать универсальным наложением компакта $X$.

ПРИмеР 7.12 (ноттингемский компакт). Если $R$ - коммутативное ассоциативное кольцо с единицей, то множество формальных степенных рядов $f(x)=$ $x+a_{2} x^{2}+a_{3} x^{3}+\cdots$ с коэффициентами $a_{i} \in R$ образует группу относительно композиции: $f g(x)=f(g(x))$. Эта группа $N(R)$ действует эффективно и $R$-линейно на кольце формальных степенных рядов $R[[x]]$. В случае $R=\mathbb{Z}_{p}$ (целые $p$-адические числа) $N(R)$ известна в алгебре как ноттингемская груп$n a$, а в случае $R=\mathbb{Z}$ она связана с алгеброй стабильных когомологических операций в комплексных кобордизмах [111], [112]. Если $R$ - топологическое кольцо, то снабжая $N(R)$ топологией произведения $\prod_{i=2}^{\infty} R$, мы получим топологическую группу. Пусть $N_{n}(R)$ - подгруппа $N(R)$, состоящая из всех формальных рядов с $a_{2}=\cdots=a_{n}=0$, и пусть $N^{n}(R)$ обозначает факторгруппу $N(R) / N_{n}(R)$. Ясно, что $N(R)$ - обратный предел отсекающих отображений $\cdots \rightarrow N^{2}(R) \rightarrow N^{1}(R)=1$.

Рассмотрим теперь топологические факторгруппы $X=N(\mathbb{R}) / N(\mathbb{Z})$ и $P_{n}=$ $N^{n}(\mathbb{R}) / N^{n}(\mathbb{Z})$. Поскольку связующие отображения в $\cdots \rightarrow N^{2}(\mathbb{Z}) \rightarrow N^{1}(\mathbb{Z})$ сюръективны, то $X-$ предел обратной последовательности $\cdots \rightarrow P_{2} \rightarrow P_{1}$.

Универсальные накрывающие $P_{i}$ суть $N^{i}(\mathbb{R})$, значит, $N(\mathbb{R})$ - универсальное обобщенное налегающее компакта $X$. Поскольку $p: N(\mathbb{R}) \rightarrow X-$ серровское расслоение, а $N(\mathbb{R})$ очевидно стягиваема, мы находим, что $\stackrel{\Delta}{\pi}_{1}(X) \simeq N(\mathbb{Z})$ (как топологические группы) и $\stackrel{\Delta}{\pi}_{i}(X)=0$ при $i>1$. (Этот факт был подмечен в [113].) Так как $p: N(\mathbb{R}) \rightarrow X$ - также и стинродовское расслоение, аналогично заключаем (cp. теорему $3.15(\mathrm{~b})$ ), что стинродовские гомотопические группы $X$ точно такие же; более того, $\stackrel{\Delta}{\tau}: \stackrel{\Delta}{\pi}_{i}(X) \rightarrow \pi_{i}(X)$ - изоморфизм для всех $i$. 
С алгебраической точки зрения это неожиданное совпадение сингулярных гомотопий со стинродовскими оказывается возможным лишь благодаря нильпотентной аппроксимируемости группы $N(Z)=\pi_{1}(X) .^{21}$

В самом деле, рассмотрим следующее вложение гавайской серьги $E$ (см. §5) в компакт $X$. Поскольку каждое отсекающее отображение $p_{n}: P_{n+1} \rightarrow P_{n}-$ расслоение со слоем $S^{1}$, мы можем вложить $(n+1)$-кратный букет окружностей $Q_{n+1}$ в $P_{n+1}$, объединяя некоторое сечение $p_{n}$ над $n$-кратным букетом $Q_{n}$ и слой $p_{n}$ над базисной точкой этого букета. Это доставляет вложение обратного предела $E$ букетов $Q_{n}$ в компакт $X$.

Напомним (см. пример 5.6), что в образе $\stackrel{\Delta}{\tau}: \stackrel{\Delta}{\pi}_{1}(E) \rightarrow \pi_{1}(E)$ не лежит, например, класс такой стинродовской петли $\ell$ :

$$
\left(\ldots,\left[a_{1}, a_{2}\right]\left[a_{1}, a_{3}\right]\left[a_{1}, a_{4}\right],\left[a_{1}, a_{2}\right]\left[a_{1}, a_{3}\right],\left[a_{1}, a_{2}\right]\right) \in \underset{\longleftarrow}{\lim }\left(\cdots \rightarrow F_{4} \rightarrow F_{3} \rightarrow F_{2}\right) .
$$

Он не представляется отображением $S^{1} \rightarrow E$ по той простой причине, что подобное отображение не может намотаться на первую окружность гавайской серьги бесконечное число раз, тогда как литера $a_{1}$ считывается конструкцией Понтрягина-Тома именно при каждом обходе первой окружности в положительном направлении.

Каков класс $\ell$ в $\pi_{1}(X)$ ? Несложно проверить, что (в группе $N(R)$ с произвольным $R$ ) если $\alpha=x+a x^{n}+\cdots$ и $\beta=x+b x^{m}+\cdots$, то $\alpha^{-1} \beta^{-1} \alpha \beta=$ $x+a b(m-n) x^{m+n-1}+\cdots$, где многоточие символизирует члены более высоких степеней, ср. [114]. ${ }^{22}$ Таким образом, благодаря соотношениям в группе $N(\mathbb{Z})$ коммутаторы $\left[a_{1}, a_{i}\right]$ попадают во все более глубокие подгруппы $N_{i}(\mathbb{Z})$ этой группы и потому представляются все более короткими (экспоненциально укорачивающимися) петлями. Так что их произведение тоже представимо петлей, как мы и ожидали.

ЗАмечАнИЕ. Заметим, между прочим, что универсальное обобщенное налегающее $\widetilde{E}$ гавайской серьги не является линейно связным. Действительно, пусть $F$ - слой стинродовского расслоения $\widetilde{E} \rightarrow E$. В обозначениях предыдущего примера, образ $[\ell]$ в $\pi_{0}(F)$ (см. теорему $\left.3.15(\mathrm{~b})\right)$ представи́м отображением $t: S^{0} \rightarrow F$ в силу нульмерности $F$. Образ же $[t]$ в $\stackrel{\Delta}{\pi}_{0}(\widetilde{E})$ нетривиален в силу выбора $\ell$ и стинродовской односвязности $\widetilde{E}$.

\section{§ 8. Нульмерные гомотопии}

В нульмерном случае теорема 3.12 допускает следующую модификацию.

ПредЛожениЕ 8.1 (Красинкевич [115; теорема 1.1]). Пусть X - предел обратной последовательности компактных полиэдров $\cdots \rightarrow P_{2} \rightarrow P_{1}$. Тогда $X$ стинродовски связен, если и только если для каждого $k$ существует такое $j>k$, что для каждого $i>j$ каждый путь $(I, \partial I) \rightarrow\left(P_{[j, \infty]}, X\right)$ гомотопен неподвижно на концах I и со значениями в $P_{[k, \infty]}$ некоторому пути в $P_{[i, \infty]}$.

\footnotetext{
21 а также благодаря асферичности $X$ - см. пример 8.6 и замечание к нему.

${ }^{22}$ Из этого равенства немедленно следует, что $N^{n}(R)$ нильпотентна.
} 
Первоначальное доказательство Красинкевича было геометрическим.

ДокАЗАТЕЛЬСтво. По теореме 3.1 и лемме $3.3 X$ стинродовски связен тогда и только тогда, когда $\pi_{1}\left(P_{i}\right)$ удовлетворяют условию Миттаг-Леффлера. По лемме 3.11 это имеет место тогда и только тогда, когда $\stackrel{\Delta}{\pi}_{1}\left(P_{[i, \infty]}, X\right)$ удовлетворяют условию Миттаг-Леффлера. Гомотопию $h$ пути можно преобразовать в неподвижную на втором конце, используя ограничение $h$ на этот конец.

Теорема 8.2 (Макмиллан [99]; Красинкевич [29; следствие 7.2.4], [115]). Непрерывный образ стинродовски связного компакта стинродовски связен.

При этом непрерывная сюръекция между компактами не обязана индуцировать эпиморфизм на $\pi_{0}$, как показывает рассмотрение проекции $\Sigma_{p} \sqcup \Sigma_{p} \rightarrow$ $\Sigma_{p} \vee \Sigma_{p}$ (см. примеры 5.3 и 5.5 ).

Приведенное доказательство упрощает подход Макмиллана благодаря использованию предложения 8.1. Оно также намного проще обоих доказательств Красинкевича. Еще одно доказательство, с использованием неметризуемых компактов, приведено в третьей статье из серии [24].

ДокАзАТЕЛьство. Пусть $f: X \rightarrow Y$ - заданная сюръекция. Используя доказательство теоремы 3.15 (а), мы можем предполагать, что

$$
X=\lim _{\longleftarrow} P_{i}, \quad Y=\lim _{\longleftarrow} Q_{i}, \quad f=\lim _{\longleftarrow} f_{i}
$$

для некоторых компактных $P_{i}$ и $Q_{i}$ и PL-отображений $f_{i}: P_{i} \rightarrow Q_{i}$, коммутирующих со связующими отображениями. Возьмем точку $v \in Y$, и пусть $v_{k}-$ ее образ в $Q_{k}$. Выберем полиэдральные окрестности $N_{k}(v)$ точки $v_{k}$ в $Q_{k}$ так, чтобы каждая $N_{k}(v)$ содержала образ $N_{k+1}(v)$ и чтобы выполнялось равенство $\lim _{i} N_{i}(v)=\{v\}$. Пусть $M_{k}(v)=f_{k}^{-1}\left(N_{k}(v)\right)$, тогда $\lim _{\longleftarrow} M_{i}(v)=f^{-1}(v)$; в частно-

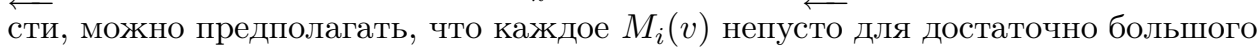
$i=i(v)$.

Согласно конструкции Ферри для компактов, удовлетворяющих L $\stackrel{\leftrightarrow}{C}_{-1}$ (см. начало $\S 6)$, для каждого $k$ существуют $j$ и $n$ такие, что путь $\ell:(I, \partial I) \rightarrow$ $\left(Q_{[j, \infty]}, Y\right)$ гомотопен неподвижно на крае со значениями в $Q_{[k, \infty]}$ пути $\ell^{\prime}$ такому, что $y_{i}:=\ell^{\prime}(i / n) \in Y$ для каждого $i=0,1, \ldots, n$ и $q_{k}^{\infty}\left(y_{i}\right) \in N_{k}\left(y_{i+1}\right)$ для каждого $i<n$. Тогда существует такое $h>k$, что $q_{k}^{h}\left(N_{h}\left(y_{i}\right)\right) \subset N_{k}\left(y_{i+1}\right)$ для каждого $i<n$. Выберем некоторую точку $x_{i} \in M_{h}\left(y_{i}\right)$ для каждого $i=0,1, \ldots, n$. Заметим, что $p_{k}^{h}\left(x_{i}\right) \in M_{k}\left(y_{i+1}\right)$. Согласно конструкции Ферри для компактов, удовлетворяющих $\mathrm{L}_{-1}$, для заданного $l$ мы можем выбрать $k$ так, что $x_{i}$ соединяется с некоторой точкой $x_{i}^{-} \in f^{-1}\left(y_{i}\right)$ путем в $M_{[l, \infty]}\left(y_{i}\right)$ и с некоторой точкой $x_{i}^{+} \in f^{-1}\left(y_{i+1}\right)$ путем в $M_{[l, \infty]}\left(y_{i+1}\right)$. Пусть $\ell_{i}:(I, \partial I) \rightarrow$ $\left(P_{[0, \infty]}, X\right)$ - конкатенация этих двух путей, соединяющая $x_{i}^{-}$с $x_{i}^{+}$. Так как $Q_{[0, \infty]}$ удовлетворяет $\stackrel{\Delta}{\mathrm{C}}_{\infty}$, то для заданного $m$ мы можем выбрать $l$ так, что $f_{[0, \infty]}\left(\ell_{i}\right)$ и $\left.\ell^{\prime}\right|_{\left[\frac{i}{n}, \frac{i+1}{n}\right]}$ гомотопны неподвижно на крае и со значениями в $Q_{[m, \infty]}$. С другой стороны, по предложению 8.1 мы можем выбрать $l=l(m)$ так, что для каждого $t$ каждый $\ell_{i}$ гомотопен неподвижно на крае и со значениями в $P_{[m, \infty]}$ некоторому пути в $P_{[t, \infty]}$. 
Теорема 8.3 (Красинкевич [115]). Связное объединение двух стинродовски связных компактов стинродовски связно.

Это утверждение - частный случай следствия 3.14, но поскольку оно будет существенно использовано ниже, мы приведем немного другое, более прямое доказательство.

ДоказАтельство. Мы можем представить $X$ как $\underset{\lim }{\longleftarrow}\left(\cdots \rightarrow P_{2} \rightarrow P_{1}\right)$, где каждое $P_{i}$ - объединение двух компактных полиэдров $Q_{i}$ и $R_{i}$ таких, что $Y=\lim _{\longleftarrow} Q_{i}$ и $Z=\lim _{\Delta} R_{i}$. Согласно конструкции Ферри для компактов, удовлетворяющих $\stackrel{\Delta}{\mathrm{C}}-1_{-}$(см. начало $\left.\S 6\right)$, для каждого $k$ существует $j$ такое, что каждый путь $l:(I, \partial I) \rightarrow\left(P_{[j, \infty]}, X\right)$ гомотопен неподвижно на крае $I$ и со значениями в $P_{[k, \infty]}$ конкатенации путей $\ell_{i}$, пролегающих поочередно в $\left(Q_{[k, \infty]}, Y\right)$ и в $\left(R_{[k, \infty]}, Z\right)$. Для заданного $l$ по предложению 8.1 число $k>l$ может быть выбрано так, что для произвольного $h>k$ каждый $\ell_{2 i+1}$ гомотопен неподвижно на крае $I$ и со значениями в $Q_{[l, \infty]}$ некоторому пути в $Q_{[h, \infty]}$ и каждый $\ell_{2 i}$ гомотопен неподвижно на крае $I$ и со значениями в $R_{[l, \infty]}$ некоторому пути в $R_{[h, \infty]}$. Если путей $\ell_{i}$ бесконечно много, можно обойтись лишь конечным количеством нетождественных гомотопий. Следовательно, $\ell$ гомотопен неподвижно на крае $I$ и со значениями в $P_{[l, \infty]}$ некоторому пути в $P_{[h, \infty]}$.

ЗАмечАниЕ. Доказательство предложения 8.1 показывает (см. замечание к лемме 3.11$)$, что образы групп $\pi_{1}\left(P_{i}\right)$ в $\pi_{1}\left(P_{k}\right)$ стабилизируются, если и только если стабилизируются (с тем же $k$ ) образы групп $\stackrel{\Delta}{\pi}_{1}\left(P_{[i, \infty]}, X\right)$ в $\stackrel{\Delta}{\pi}_{1}\left(P_{[k, \infty]}, X\right)$. В таком случае назовем связный компакт $X$ стинродовски связным над $P_{k}$ (или, более подробно, над $p_{k}^{\infty}: X \rightarrow P_{k}$ ). Легко видеть, что наличие этого свойства не зависит от выбора $P_{k+1}, P_{k+2}, \ldots$.

По теореме 3.1 (b) и лемме 3.3 компакт стинродовски связен тогда и только тогда, когда он стинродовски связен над всяким отображением в полиэдр. По доказательству теоремы 8.3 имеем, что если $X$ - объединение компактов $Y$ и $Z$ и $f: X \rightarrow P$ - такое отображение в полиэдр, что $Y$ и $Z$ стинродовски связны над $\left.f\right|_{Y}$ и $\left.f\right|_{Z}$, то $X$ стинродовски связен над $f$.

Говорят, что обратная последовательность групп $G_{i}$ удовлетворяет виртуалъному условию Миттаг-Леффлера, если для каждого $i$ найдется такое $j>i$, что для всякого $k>j$ образ отображения $G_{k} \rightarrow G_{i}$ имеет конечный индекс в образе $G_{j} \rightarrow G_{i}$. Если $X$ - предел обратной последовательности компактных связных полиэдров $P_{i}$, выполнение для обратной последовательности $\pi_{1}\left(P_{i}\right)$ виртуального условия Миттаг-Леффлера, очевидно, не зависит от выбора базисной точки $X$ и является инвариантом отношения эквивалентности из предложения 2.6 (iii). Значит, мы можем назвать $X$ виртуально связным по Стинродy, если $\pi_{1}\left(P_{i}\right)$ удовлетворяют виртуальному условию Миттаг-Леффлера; причем свойство быть виртуально связным по Стинроду является (непунктированным) шейповым инвариантом.

Например, $\cdots \stackrel{f}{\rightarrow} F_{2} \stackrel{f}{\rightarrow} F_{2}$, где $F_{2}$ - свободная группа $\langle x, y \mid\rangle$ и $f(x)=x^{p}$, $f(y)=y$, не удовлетворяет виртуальному условию Миттаг-Леффлера. В частности, букет $p$-адического соленоида и $S^{1}$ не является виртуально связным по Стинроду. 
Теорема 8.4 (Гэган-Красинкевич [49]). Если связный компакт $X$ не является виртуалъно связным по Стинроду, то отображение $\stackrel{\Delta}{\pi}_{0}(X) \stackrel{\stackrel{\Delta}{\rightarrow}}{\rightarrow} \pi_{0}(X)$ не сторгективно.

Мы модифицируем доказательство из [49], заменяя некоторые излишние построения алгебраическим анализом компактифицированных телескопов, который пригодится в оставшейся части настоящего параграфа.

ДоКАЗАТЕЛЬство. Предположим, что $X$ - предел обратной последовательности компактных связных полиэдров $P_{i}$, и пусть $G_{i}=\pi_{1}\left(P_{i}\right)$. По условию существует $k$ такое, что образы $A_{i}$ групп $G_{k+i}$ в $G_{k}$ не стабилизируются виртуально, т. е. пунктированные множества $A_{i} / A_{i+1}$ бесконечны для бесконечного количества значений $i$. Тогда если $A:=\lim A_{0} / A_{i}$ снабжено топологией обратного предела (где каждое $A_{0} / A_{i}$ дискретно), то каждый компакт в $A$ нигде не плотен в $A$ (подробности аналогичны приводимым в доказательстве леммы 3.7$)$. Поскольку $A$ полно, по теореме Бэра о категории $A$ не является счетным объединением компактов.

Оставшаяся часть доказательства излагается в двух вариантах.

ПРОСТОЕ РАССУЖДЕНИЕ (с использованием стинродовских гомотопий равномерных пространств, см. §7). Если $K-$ компакт, то $\stackrel{\Delta}{\pi}_{0}(K)$ компактно, поскольку $K$ является непрерывным образом канторова множества. Пусть $\widetilde{X} \rightarrow X-$ пулбек (обратный образ) универсального накрытия $\widetilde{P}_{k} \rightarrow P_{k}$. Тогда $\widetilde{X}$ - счетное объединение компактов. В частности, $\stackrel{\pi}{0}_{0}(\widetilde{X})$ - счетное объединение компактов.

Пусть $\widetilde{P}_{k+i}-$ пулбек $\widetilde{P}_{k}$ над $P_{k+i}$. Тогда обратная последовательность $\cdots \rightarrow \widetilde{P}_{1} \rightarrow \widetilde{P}_{0}$ сходится, с обратным пределом $\widetilde{X}$. Легко видеть, что $\pi_{0}\left(\widetilde{P}_{k+i}\right)$ можно отождествить с $A_{0}$-множеством $A_{0} / A_{i}$, и, следовательно,

$$
\check{\pi}_{0}(\widetilde{X}):=\lim _{\longleftarrow} \pi_{0}\left(\widetilde{P}_{i}\right)
$$

является $A_{0}$-гомеоморфным компакту $A$. Поскольку $\check{\tau}: \pi_{0}(\widetilde{X}) \rightarrow \check{\pi}_{0}(X)$ является сюръекцией аналогично теореме $3.1(\mathrm{~b})$, то $\pi_{0}(\tilde{X})$ не является счетным объединением компактов.

Значит, $\stackrel{\Delta}{\tau}: \stackrel{\Delta}{\pi}_{0}(\widetilde{X}) \rightarrow \pi_{0}(\tilde{X})$ не сюръективно. Но из точных гомотопических последовательностей стинродовского расслоения (аналогично теореме $3.15(\mathrm{~b})$ ) и серровского расслоения следует, что это отображение является $A_{0}$-эквивариантным поднятием отображения $\stackrel{\Delta}{\tau}: \stackrel{\Delta}{\pi}_{0}(X) \rightarrow \pi_{0}(X)$. Стало быть, и последнее - не сюръекция.

ЭЛЕМЕНтАРноЕ РАССУЖДЕНИЕ. Базисная точка $x \in X$ порождает базисный луч $p_{[k, \infty)}$ в $P_{[k, \infty)}$. Так как каждое $P_{[i, \infty)}$ деформационно ретрагируется на $P_{i}$, то каждое $A_{0} / A_{i}$ можно отождествить, как $A_{0}$-множество, с $\pi_{1}\left(P_{[k, \infty)}\right.$, $\left.P_{[i, \infty)} ; p_{k}\right)$. Аналогично доказательству теоремы $3.1(\mathrm{~b})$, это доставляет сюръекцию $\varphi: G \rightarrow A$, где $G$ - пунктированное множество собственных гомотопических классов собственных отображений $([0, \infty), 0) \rightarrow\left(P_{[k, \infty)}, p_{k}\right) .^{23}$ Тогда

\footnotetext{
${ }^{23}$ По лемме $3.13 G$ можно отождествить с $\pi_{1}\left(P_{[k, \infty]}, X\right)$.
} 
пространство орбит $A_{0} \backslash G$ левого действия $A_{0}$ на $G$ может быть отождествлено с $\pi_{0}(X){ }^{24}$ Из точной гомотопической последовательности пары следует, что $\stackrel{\Delta}{\pi}_{0}(X ; x)$ является пространством орбит $A_{0} \backslash H$ левого действия $A_{0} \simeq$ $\stackrel{\Delta}{\pi}_{1}\left(P_{[k, \infty]} ; x\right)$ на $H:=\stackrel{\Delta}{\pi}_{1}\left(P_{[k, \infty]}, X ; x\right)$. Используя гомотопию $\Pi_{t}$ из $\S 2$, каждое $h \in H$ можно представить отображением $[-\infty, \infty] \rightarrow P_{[k, \infty]}$, отправляющим $[-\infty, 0]$ гомеоморфно в $p_{[k, \infty]}$ и $[0, \infty)$ в $P_{[k, \infty)}$; и любые два таких представителя $h$ гомотопны в классе таких отображений. Это доставляет $A_{0}$-эквивариантное отображение $\psi: H \rightarrow G$ такое, что отображение пространств орбит $A_{0} \backslash H \rightarrow$ $A_{0} \backslash G$ можно отождествить с $\stackrel{\Delta}{\tau}: \stackrel{\Delta}{\pi}_{0}(X) \rightarrow \pi_{0}(X)$. В частности, если $\stackrel{\Delta}{\tau}$ сюръективно, то $\psi$ тоже сюръективно. Топологизируем $H$ и $G$, объявив базисными открытыми множествами прообразы точек отображений в $\stackrel{\Delta}{1}_{1}\left(P_{[k, \infty]}, P_{[i, \infty]}\right) \simeq$ $\pi_{1}\left(P_{[k, i]}, P_{i}\right)$, соответственно в $\stackrel{\Delta}{\pi}_{1}\left(P_{[k, \infty)}, P_{[i, \infty)}\right) \simeq \pi_{1}\left(P_{[k, i]}, P_{i}\right)$. Тогда $\psi$ и $\varphi$ непрерывны.

Пусть $T_{1}, T_{2}, \ldots$ - некоторые триангуляции $P_{1}, P_{2}, \ldots$ такие, что каждое связующее отображение $P_{k+1} \rightarrow P_{k}$ симплициально относительно $T_{k+1}$ и некоторого измельчения барицентрического измельчения триангуляции $T_{k}$. Если $Q_{k}$ обозначает двойственный 0 -мерный остов $T_{k}$, то, относя каждому симплексу старшей размерности $\sigma$ триангуляции $T_{k+1}$ симплекс триангуляции $T_{k}$, содержащий образ $\sigma$, мы получаем сюръекцию $Q_{k+1} \rightarrow Q_{k}$ с конечными прообразами точек. Предел обратной последовательности $Q_{k}$ является канторовым множеством $C$, и включение $Q_{[0, \infty)} \subset P_{[0, \infty)}$ продолжается до непрерывного отображения $Q_{[0, \infty]} \rightarrow P_{[0, \infty]}$, отправляющего $C$ на $X$. Более того, если ввести обозначение $K=\stackrel{\Delta}{\pi}_{1}\left(Q_{[k, \infty]} \cup P_{k}, C\right)$, несложно видеть, что индуцированное непрерывное отображение $\chi: K \rightarrow H$ сюръективно. Наконец, $K$ - предел обратной последовательности дискретных пространств $\pi_{1}\left(Q_{[k, i]} \cup P_{k}, Q_{i}\right)$, где связующие отображения имеют конечные прообразы точек. Таким образом, $K-$ счетное объединение компактов. Так как композиция $K \stackrel{\chi}{\rightarrow} H \stackrel{\psi}{\longrightarrow} G \stackrel{\varphi}{\longrightarrow} A$ является непрерывной сюръекцией, то $A$ должно быть счетным объединением компактов, что ведет к противоречию.

Лемма 8.5 (Красинкевич-Гэган [116; с. 48], [49; §7]). Пусть X - предел обратной последователъности компактных связных полиэдров $P_{i}$ и связующих отображений $p_{i}: P_{i+1} \rightarrow P_{i}$. Пусть $b \in X-$ базисная точка $u b_{i} \in P_{i}-e е$ образы.

Класс а элемента $\left(g_{0}, g_{1}, \ldots\right) \in \prod \pi_{1}\left(P_{i}\right)$ в $\lim ^{1} \pi_{1}\left(P_{i}, b_{i}\right)=\pi_{0}(X, b)$ представлен в точности теми точками $X$, которые лежат в образе проекции обратного предела $W_{\alpha}$ универсальных накрывающих $\cdots \stackrel{\tilde{p}_{1}}{\longrightarrow} \widetilde{P}_{1} \stackrel{g_{1}}{\longrightarrow} \widetilde{P}_{1} \stackrel{\tilde{p}_{0}}{\longrightarrow}$ $\widetilde{P}_{0} \stackrel{g_{0}}{\longrightarrow} \widetilde{P}_{0}$, где каждое $\tilde{p}_{i}$ переводит фиксированное поднятие $\tilde{b}_{i+1}$ отображения $b_{i+1}$ в предъдущее $\tilde{b}_{i}$.

В частности, $\alpha$ лежст в образе $\stackrel{\Delta}{\pi}_{0}(X, b)$, если и только если $W_{\alpha}$ непуст.

Мы называем множество точек компакта $X$, представляющих некоторый элемент $\alpha \in \pi_{0}(X)$, стинродовской компонентой $X$. В этой терминологии

\footnotetext{
${ }^{24} \mathrm{~B}$ действительности, из доказательства леммы 3.3 следует, что $\varphi-A_{0}$-эквивариантное поднятие сюръекции $\lim ^{1} G_{i} \rightarrow \lim ^{1} A_{i}$, индуцированной сюръекциями $G_{i} \rightarrow A_{i}$.
} 
пример 5.5 и теорема 8.4 утверждают существование пустых стинродовских компонент.

ДокАЗАтЕльство. Предположим, что $\left(g_{0}, g_{1}, \ldots\right) \in \prod \pi_{1}\left(P_{i}\right)$ представляет образ в $\pi_{0}(X, b)$ некоторого $[a] \in \stackrel{\Delta}{\pi}_{0}(X, b)$, где $a \in X$ - точка. Рассмотрим ее образы $a_{i} \in P_{i}$ и зафиксируем путь $w_{i}$ из $b_{i}$ в $a_{i}$. Тогда $\left(g_{0}, g_{1}, \ldots\right)$ представляет тот же элемент из $\lim ^{1} \pi_{1}\left(P_{i}, b_{i}\right)$, что и $\left(g_{0}^{\prime}, g_{1}^{\prime}, \ldots\right)$, где $g_{i}^{\prime}-$ класс петли $w_{i} p_{i+1}\left(\bar{w}_{i+1}\right)$. Мы можем предполагать, что $g_{i}^{\prime}=g_{i}$, переопределив подходящим образом пути $w_{i}$ исходя из определения $\lim ^{1}$. Рассмотрим поднятие $\widetilde{w}_{i}$ каждого $w_{i}$ с началом в $\tilde{b}_{i}$, и пусть $\tilde{a}_{i}-$ другой его конец. Тогда композиция $\widetilde{P}_{i+1} \stackrel{\tilde{p}_{i}}{\longrightarrow} \widetilde{P}_{i} \stackrel{g_{i}}{\longrightarrow} \widetilde{P}_{i}$ переводит $\tilde{a}_{i+1}$ в точности в $\tilde{a}_{i}$. Следовательно, обратный предел этих композиций содержит обратный предел одноэлементных множеств $\left\{\tilde{a}_{i}\right\}$, который непуст.

Обратно, для любой точки $\tilde{a}$ обратного предела композиций $\widetilde{P}_{i+1} \stackrel{\tilde{p}_{i}}{\longrightarrow} \widetilde{P}_{i} \stackrel{g_{i}}{\longrightarrow} \widetilde{P}_{i}$, обратив предыдущее рассуждение, можно показать, что проекция $a$ точки $\tilde{a}$ на $X$ представляет класс последовательности $\left(g_{0}, g_{1}, \ldots\right)$ в группе $\pi_{1}(X)$.

ЗАмечАниЕ. Аналогично лемме 8.5 доказывается ее гомологическая версия, которая может быть использована, например, для прояснения алгебраического содержания примеров 5.4 и 5.5. А именно, она утверждает, что класс $\alpha$ элемента $\left(g_{0}, g_{1}, \ldots\right) \in \prod H_{1}\left(P_{i}\right)$ в $\lim ^{1} H_{1}\left(P_{i}\right)=H_{0}(X, b)$ представлен в точности теми точками $X$, которые лежат в образе проекции обратного предела $W_{\alpha}$ универсальных абелевых накрывающих $\cdots \stackrel{\tilde{p}_{1}}{\longrightarrow} \widetilde{P}_{1} \stackrel{g_{1}}{\longrightarrow} \widetilde{P}_{1} \stackrel{\tilde{p}_{0}}{\longrightarrow} \widetilde{P}_{0} \stackrel{g_{0}}{\longrightarrow} \widetilde{P}_{0}$, где каждое $\tilde{p}_{i}$ переводит фиксированное поднятие $\tilde{b}_{i+1}$ отображения $b_{i+1}$ в предыдущее $\tilde{b}_{i}$. В частности, $\alpha$ лежит в образе композиции $\stackrel{\Delta}{\pi}_{0}(X, b) \rightarrow \stackrel{\Delta}{H}_{0}(X) \rightarrow H_{0}(X)$, если и только если $W_{\alpha}$ непуст.

Дословное обращение теоремы 8.4 не имеет места, как показывает следующий пример.

ПРимеР 8.6 (компакт Брина ${ }^{25}$ ). Пусть $X \subset \Sigma_{p} \times \Sigma_{p} \times \Sigma_{p}$ определен как объединение $\left[\Sigma_{p} \times \Sigma_{p} \times \mathrm{pt}\right] \cup\left[\Sigma_{p} \times \mathrm{pt} \times \Sigma_{p}\right] \cup\left[\mathrm{pt} \times \Sigma_{p} \times \Sigma_{p}\right]$. Таким образом, $X=\lim _{\longleftarrow}(\cdots \stackrel{f}{\rightarrow} P \stackrel{f}{\rightarrow} P)$, где $P=\left[S^{1} \times S^{1} \times \mathrm{pt}\right] \cup\left[S^{1} \times \mathrm{pt} \times S^{1}\right] \cup\left[\mathrm{pt} \times S^{1} \times S^{1}\right]$ и $f$ - ограничение отображения $p \times p \times p$ пространства $S^{1} \times S^{1} \times S^{1}$ в себя. Так как включение $P \subset\left(S^{1}\right)^{3}$ индуцирует изоморфизм на $\pi_{1}$, то включение $X \subset\left(\Sigma_{p}\right)^{3}$ индуцирует биекцию на $\pi_{0}$. В частности, $X$ виртуально связен по Стинроду.

Пусть $\alpha=\overline{\ldots \alpha_{1} \alpha_{0}} \in \mathbb{Z}_{p} \backslash \mathbb{Z}$. Мы утверждаем, что $g=(\alpha+\mathbb{Z}, \alpha+\mathbb{Z}, \alpha+\mathbb{Z}) \in$ $\left(\mathbb{Z}_{p} / \mathbb{Z}\right)^{3} \simeq \pi_{0}(X)$ не лежит в образе $\stackrel{\Delta}{\pi}_{0}(X)$. Действительно, универсальное накрывающее $\widetilde{P}$ полиэдра $P$ можно отождествить с рассмотренной как пространство подгруппой $[\mathbb{R} \times \mathbb{R} \times \mathbb{Z}] \cup[\mathbb{R} \times \mathbb{Z} \times \mathbb{R}] \cup[\mathbb{Z} \times \mathbb{R} \times \mathbb{R}]$ топологической группы $\mathbb{R}^{3}$. Пересечение вложенной последовательности

$$
\cdots \subset\left(\overline{\alpha_{1} \alpha_{0}}, \overline{\alpha_{1} \alpha_{0}}, \overline{\alpha_{1} \alpha_{0}}\right)+p^{2} \widetilde{P} \subset\left(\overline{\alpha_{0}}, \overline{\alpha_{0}}, \overline{\alpha_{0}}\right)+p \widetilde{P} \subset \widetilde{P}
$$

пусто. Следовательно, по лемме $8.5 \mathrm{~g}$ не лежит в образе $\stackrel{\Delta}{\pi}_{0}(X)$.

\footnotetext{
25 Это может быть, но может и не быть тем самым компактом Брина, который упомянут в [49; замечание 10.4].
} 
ЗАмЕчАниЕ. Достаточные условия сюръективности отображения $\stackrel{\Delta}{\pi}_{0}(X) \stackrel{\stackrel{\Delta}{\tau}}{\rightarrow}$ $\pi_{0}(X)$, включающие в себя условие конечности $\mathrm{FP}_{n}$ и $n$-связность на бесконечности фундаментальных групп нервов $X$, найдены в [49; $\S 6-10]$. Согласно [49; замечание 10.3], Ферри доказал следующее обращение теоремы 8.4: каждый виртуально связный по Стинроду компакт (в частности, компакт Брина!) стинродовски гомотопически эквивалентен компакту $X$ такому, что $\stackrel{\Delta}{\pi}_{0}(X) \rightarrow \pi_{0}(X)$ сюръективно.

Теорема 8.7 (Красинкевич-Минц [117]). Пусть $X$ - связный компакт, не связный по Стинроду. Тогда образ $\stackrel{\Delta}{\tau}: \stackrel{\Delta}{\pi}_{0}(X) \rightarrow \pi_{0}(X)$ несчетен (в частности, нетривиален).

Приведенное доказательство существенно упрощает исходное [117; с. 147-151] (продолженное из [115; с. 146-147]), которое опиралось на теорию континуумов (используя по существу композанты и неразложимые континуумы). Отметим, что теорема 8.7 влечет теоремы 8.2 и 8.3 .

ДоКАЗАТЕЛЬСтво. Пусть $\cdots \stackrel{p_{2}}{\longrightarrow} P_{2} \stackrel{p_{1}}{\longrightarrow} P_{1}$ - обратная последовательность полиэдров с $\lim P_{i}=X$ такая, что $X$ стинродовски несвязен над $P_{1}$ (см. определение в замечании к теореме 8.3$)$. Пусть $G_{i}$ - образ $\pi_{1}\left(P_{i}\right)$ в $\pi_{1}\left(P_{1}\right)$. Пусть $B G_{1}-$ классифицирующее пространство группы $\pi_{1}\left(P_{1}\right)$ (локально компактный полиэдр гомотопического типа $\left.K\left(G_{1}, 1\right)\right)$, и пусть $B G_{i}$ - накрывающее пространства $B G_{1}$, соответствующее $G_{i}$. Тогда связующие отображения $p_{1}^{i}: P_{i} \rightarrow P_{1}$ поднимаются до отображений $f_{i}: P_{i} \rightarrow B G_{i}$, коммутирующих с $p_{i}$ и с накрытиями $q_{i}: B G_{i+1} \rightarrow B G_{i}$. Они сходятся в отображение $f$ из $X$ в обратный предел $B G$ пространств $B G_{i}$. Пусть $Q_{i}$ - объединение всех (замкнутых) симплексов $B G_{i}$, пересекающих $f_{i}\left(P_{i}\right)$, где $B G_{i}$ триангулировано произвольно при $i=1$ и так, что $q_{i-1}$ симплициально, при $i>1$. Заметим, что каждое $Q_{i}$ компактно, так как компактно $f_{i}\left(P_{i}\right)$. Каждое индуцированное включением отображение $\pi_{1}\left(Q_{i}\right) \rightarrow G_{i}$ является эпиморфизмом, поскольку $f_{i}$ пропускается через $Q_{i}$. Следовательно, $Y:=\lim Q_{i}$ стинродовски несвязен над $Q_{1}$.

По лемме 8.5 каждая стинродовская компонента $\alpha$ пространства $B G$ является образом обратного предела $W_{\alpha}$ автогомеоморфизмов универсального накрытия $E G_{1}$ пространства $B G_{1}$. Если $E G_{1}$ триангулировано так, что проекция $E G_{1} \rightarrow B G_{1}$ симплициальна, мы получаем, что $\alpha$ является непрерывным образом триангулированного полиэдра $W_{\alpha}$, причем композиция $W_{\alpha} \rightarrow \alpha \subset$ $B G \rightarrow B G_{i}$ симплициальна. В частности, отсюда следует, что $\alpha$ содержит лишь счетное количество точек обратного предела $B G^{(0)}$ нульмерных остовов $B G_{i}^{(0)}$. C другой стороны, каждая точка обратного предела $Y^{(0)}$ нульмерных остовов $Q_{i}^{(0)}$ лежит в той же стинродовской компоненте $B G$, что и некоторая точка из $f(X)$. Действительно, "замкнутая звезда" $S$ каждой вершины $y \in Y^{(0)}$ (т. е. образ звезды поднятия $y$ в триангулированный полиэдр $W_{\alpha}$, где $\left.y \in \alpha\right)$ является обратным пределом звезд $S_{i}$ вершин $q_{i}^{\infty}(y)$. Каждое пересечение $S_{i} \cap f_{i}\left(P_{i}\right)$ непусто, значит, непуст и их обратный предел $S \cap f(X)$.

Предположим, что $Y^{(0)}$ счетен. Тогда по теореме Бэра о категории (следствие 7.11) он содержит изолированную точку $v$. Удаляя "открытую звезду" вершины $v$ из $Y_{0}:=Y$, мы получаем компакт, имеющий конечное ко- 
личество компонент связности согласно когомологической последовательности Майера-Вьеториса (с использованием того, что "линк" вершины $v$ - компактный полиэдр). Хотя бы одна из них стинродовски несвязна над $Q_{0}$ по замечанию к теореме 8.3. Эта компонента $Y_{1}$ имеет нульмерный остов $Y_{1}^{(0)}:=Y_{1} \cap Y^{(0)}$, который снова содержит изолированную точку, так что рассуждение может быть повторено. Тогда $Y_{\omega}:=\bigcap_{i<\omega} Y_{i}$ - предел обратной последовательности включений $\cdots \subset Y_{1} \subset Y_{0}$. Следовательно, $Y_{\omega}$ связен (в силу непрерывности когомологий Понтрягина) и гомеоморфен пределу “лестничной” обратной последовательности $\cdots \stackrel{p_{11}}{\longrightarrow} P_{11} \stackrel{f_{11}}{\longrightarrow} P_{01} \stackrel{p_{00}}{\longrightarrow} P_{00}$, где каждое $Y_{i}$ есть $\stackrel{\lim }{\longleftarrow}\left(\cdots \stackrel{p_{i 1}}{\longrightarrow}\right.$ $\left.P_{i 1} \stackrel{p_{i 0}}{\longrightarrow} P_{i 0}=P\right)$ и каждое $f_{i,[0, \infty)}: P_{i+1,[0, \infty)} \rightarrow P_{i,[0, \infty)}$ является сохраняющим уровни отображением, представляющим стинродовский гомотопический класс включения $Y_{i+1} \subset Y_{i}$. Следовательно, каждое $\Gamma_{i}:=\operatorname{im}\left[\pi_{1}\left(P_{i i}\right) \rightarrow \pi_{1}(P)\right]$ собственно содержит $\Gamma_{i+1}$, поэтому $Y_{\omega}$ стинродовски несвязен над $Q_{0} \cdot{ }^{26}$

Наконец, $Y_{\omega}^{(0)}:=Y_{\omega} \cap Y^{(0)}$ снова счетен, и процесс может быть продолжен трансфинитной (счетной) индукцией до тех пор, пока он не остановится, т. е. пока мы не дойдем до шага $\lambda$, на котором $Y_{\lambda}$ пуст. Получаем противоречие, поскольку пустое множество $Y_{\lambda}$ не может быть стинродовски несвязным над $Q_{0}$. Значит, $Y^{(0)}$ несчетен. Так как каждая стинродовская компонента $B G$ содержит только счетное количество точек $B G^{(0)}$, получаем, что $Y^{(0)}$ пересекает несчетное количество стинродовских компонент $B G$. Так как каждая точка $Y^{(0)}$ содержится в одной стинродовской компоненте $B G$ с некоторой точкой $f(X)$, то образ композиции $\stackrel{\Delta}{\pi}_{1}(X) \rightarrow \stackrel{\Delta}{\pi}_{1}(B G) \rightarrow \pi_{1}(B G)$ несчетен. Поскольку эта композиция пропускается через $\pi_{1}(X)$, то образ $\stackrel{\Delta}{\tau}: \stackrel{\Delta}{\pi}_{1}(X) \rightarrow \pi_{1}(X)$ несчетен.

ЗАмечание. Доказательство сохранит силу, если отображение $f: X \rightarrow B G$ заменить на некоторое отображение из $Z=\lim \Gamma_{i} / G_{1}$ в $B G$, где каждый $\Gamma_{i}-$ граф Кэли действия некоторого конечного множества образующих $G_{i}$ на $G_{1}$ (который несвязен, если только $G_{i}$ отлична от $G_{1}$ ). Конкретнее, это $Z$ можно построить следующим образом. Пусть $D \subset E G_{1}-$ (замкнутая) фундаментальная область действия $G_{1}$. Пусть $\tilde{X} \rightarrow X-$ пулбек $E G_{1} \rightarrow B G_{1}$. Поскольку $\widetilde{X} \rightarrow E G_{1}$ пропускается через универсальное накрывающее $\widetilde{P}_{1}$ полиэдра $P_{1}$ и $\widetilde{X} \rightarrow \widetilde{P}_{1}$ собственно, то фундаментальная область $\widehat{D}$ действия $G_{1}$ на $\widetilde{X}$ компактна. Тогда $\check{\pi}_{0}(\widehat{D})$ тоже компактна; компактен и ее образ $Z^{(0)}$ в $\check{\pi}_{0}(\widetilde{X}):=\lim \pi_{0}\left(\widetilde{P}_{i}\right)$. При действии $G_{1}$ на $\check{\pi}_{0}(\widetilde{X})$ каждая образующая $g_{i}$ группы $G_{1}$ переводит $Z^{(0)}$ в свой экземпляр $g_{i} Z^{(0)}$. В частности, это доставляет отображение $Z^{(0)} \cap g_{i}^{-1} Z^{(0)} \rightarrow Z^{(0)}$, которое можно рассматривать как частичное отображение $h_{i}$ из $Z^{(0)}$ в себя. Пусть $Z$ - объединение торов всех отображений $h_{i}$, соответствующих фиксированному набору образующих $G_{1}$; поскольку этот набор можно считать конечным, $Z$ компактно.

ЗАмечАниЕ. Теорема 8.7 имеет гомологический аналог: если $X$ связен, но $H_{0}(X) \neq 0$, то образ $\stackrel{\Delta}{\tau}: \stackrel{\Delta}{H}_{0}(X) \rightarrow H_{0}(X)$ несчетен. По сравнению с тео-

\footnotetext{
26 Заметим, что пересечение вложенной последовательности стинродовски несвязных компактов не обязательно стинродовски несвязно. Например, пересечение вложенной последовательности гроздей соленоидов может быть одной точкой.
} 
ремой 8.7, это утверждение намного проще; оно проверяется сравнением $X$ c $l$-адическим соленоидом ( $l$ - последовательность простых чисел). Предоставляем восполнение подробностей читателю.

Теперь мы готовы доказать обещанный нульмерный аналог следствия 6.6.

СледСтвиЕ 8.8. Компакт $X$ удовлетворяет условию полу-LC $\mathrm{C}_{0}$, если и только если $\pi_{0}(X)$ дискретно.

ДокАзАтельство. Утверждение "если” следует из леммы 3.4 (b). Обратно, зафиксируем метрику на $X$ и выберем $\varepsilon>0$ так, чтобы любые две $\varepsilon$-близкие точки $X$ представляли один и тот же стинродовский гомотопический класс. Очевидно, $\check{\pi}_{0}(X)$ дискретно. Если $C$ - компонента $X$, то любая пара точек $p, q \in C$ связана цепочкой $p=p_{0}, p_{1}, \ldots, p_{n}=q$, где каждая $p_{i+1} \quad \varepsilon$-близка к $p_{i}$. Тогда все точки $C$ представляют один и тот же стинродовский гомотопический класс. По теореме $8.7 C$ стинродовски связна.

ЗАмечАНИЕ. Если рассмотреть $\stackrel{\Delta}{\pi}_{n}(X)$ как $\stackrel{\Delta}{\pi}_{0}\left(\Omega^{n} X\right)$, где $\Omega^{n} X$ - итерированное пространство петель $X$ (которое является сепарабельным метризуемым полным равномерным пространством), то доказательство теоремы 8.7 может, по всей видимости, быть использовано, чтобы показать, что если $X$ удовлетворяет $\mathrm{LC}_{n-1}$ и $\mathrm{UV}_{1}$ и $\operatorname{ker}\left[\pi_{n}(X) \stackrel{\check{\tau}}{\rightarrow} \check{\pi}_{n}(X)\right]$ нетривиально, то образ $\operatorname{ker}\left[\stackrel{\Delta}{\pi}_{n}(X) \stackrel{\check{\tau} \stackrel{\Delta}{\tau}}{\longrightarrow} \check{\pi}_{n}(X)\right]$ в $\pi_{n}(X)$ несчетен. (При $n \geqslant 2$ это утверждение также следует из теорем 6.5 и 6.1 и несчетности всякого нетривиального производного предела счетных групп, которая в свою очередь следует из леммы 3.3.) Подробности, будем надеяться, появятся позже (см. также лемму $7.9(\mathrm{~g}))$.

Теорема 8.9. Связный компакт $X$ связен по Стинроду, если и толъко если всякое налегающее пространство над $X$ имеет счетное количество компонент равномерной связности.

Доказательство продолжает рассмотрения, начатые в доказательстве теоремы 8.7 .

ДокАЗАтЕльство. Зафиксируем отображение $f: X \rightarrow P$, где $P$ - полиэдр. Мы покажем, что $X$ стинродовски связен над $P$ (см. определение в замечании к теореме 8.3 ), если и только если пулбек $\widetilde{X}$ универсального накрывающего $\widetilde{P}$ полиэдра $P$ имеет счетное количество компонент равномерной связности.

Можно предполагать, что $X=\underset{\lim }{\longleftarrow}\left(\cdots \rightarrow P_{2} \rightarrow P_{1}=P\right)$, где $P_{i}$ - компактные полиэдры. Пусть $\widetilde{P}_{i}$ - пулбек $\widetilde{P}$ над $P_{i}$. Тогда $G:=\pi_{1}(P)$ действует на компонентах $\widetilde{P}_{i}$ со стабилизатором, равным $\operatorname{im}\left(G_{i} \rightarrow G\right)$, где $G_{k}=\pi_{1}\left(P_{i}\right)$. Если $X$ стинродовски связен над $P$, пусть $k$ таково, что $\operatorname{im}\left(G_{k} \rightarrow G\right)=\operatorname{im}\left(G_{i} \rightarrow G\right)$ для каждого $i>k$. Тогда $G$-эквивариантное отображение $\pi_{0}\left(\widetilde{P}_{i}\right) \rightarrow \pi_{0}\left(\widetilde{P}_{k}\right)$ биективно для всех $i>k$. Следовательно, $\check{\pi}_{0}(\widetilde{X})$ счетно. С другой стороны,

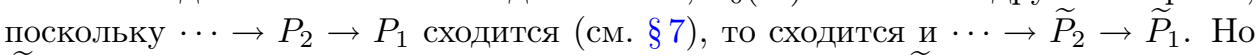
$\widetilde{X}$ - равномерно дизъюнктное объединение прообразов $\widetilde{X}^{c}$ компонент связности $\widetilde{P}_{k}^{c}$ полиэдра $\widetilde{P}_{k}$. Значит, если $\widetilde{P}_{i}^{c}$ обозначает соответствующую компоненту $\widetilde{P}_{i}$ при $i>k$, обратная последовательность $\cdots \rightarrow \widetilde{P}_{k+1}^{c} \rightarrow \widetilde{P}_{k}^{c}$ сходится. Тогда по лемме 7.9 (f) ее обратный предел $\widetilde{X}^{c}$ равномерно связен. Значит, множество равномерных компонент $\widetilde{X}$ инъективно отображается в $\check{\pi}_{0}(\widetilde{X})$. 
Обратно, предположим, что $X$ стинродовски несвязен над $P$; будем использовать обозначения из доказательства теоремы 8.7. Универсальное накрытие $\widetilde{P} \rightarrow P$ индуцировано из $E G_{1} \rightarrow B G_{1}$ отображением $f_{1}$. Пусть $\widetilde{Q}_{i}$ и $\tilde{Y}-$ пулбеки $E G_{1}$ над $Q_{i}$ и $Y$. Поскольку каждый $Q_{i}$ вложен в накрывающее пространство $\left(q_{1}^{i}\right)^{-1}\left(Q_{1}\right) \subset B G_{i}$ полиэдра $Q_{1}$, отображение $\widetilde{Q}_{i} \rightarrow \widetilde{Q}_{1}$ вкладывает каждую компоненту $\widetilde{Q}_{i}$. С другой стороны, так как $Y^{(0)}$ несчетно по доказанному ранее, то существует $x \in Q_{1}^{(0)}$, прообраз которого $\left(q_{1}^{\infty}\right)^{-1}(x)$ в $Y^{(0)}$ несчетен. Возьмем какое-нибудь поднятие $\tilde{x} \in \widetilde{Q}_{1}$, тогда его прообраз в $\widetilde{Y}$ несчетен, и в то же время точки его прообраза в каждом $\widetilde{Q}_{i}$ лежат в разных компонентах $\widetilde{Q}_{i}$. Значит, $\widetilde{Y}$ имеет несчетное количество равномерных компонент. Однако, по доказательству теоремы 8.7, $f: X \rightarrow Y$ индуцирует сюръекцию на компонентах линейной связности. Следовательно, то же можно сказать и о его поднятии $\tilde{f}: \widetilde{X} \rightarrow \widetilde{Y}$.

ЗАмечАниЕ. Теорема 8.9 влечет теорему 8.7, с использованием того, что для всякого наложения $f: E \rightarrow B$ каждый прообраз точки при $f_{*}: \pi_{0}(E) \rightarrow \pi_{0}(B)$ счетен. В самом деле, прообраз стинродовского гомотопического класса базисной точки счетен аналогично теореме 3.15 (b); но, как следует из доказательства теоремы 3.15 (b), мы имеем право взять любой стинродовский гомотопический класс $b: \mathrm{pt} \rightsquigarrow B$, являющийся композицией некоторого $\tilde{b}: \mathrm{pt} \rightarrow E$ и наложения $f$, в качестве "базисной точки".

Как было замечено в $\S 3$, обратный предел серровских расслоений $E_{i} \rightarrow B_{i}$ не обязан быть серровским расслоением; однако обратный предел композиций $f_{i}$ серровских расслоений с пулбеками предыдущих композиций $f_{i-1}$ является серровским расслоением. В частности, всякий обратный предел накрытий - серровское расслоение (независимо от того, сходится ли обратная последовательность их тотальных пространств). С другой стороны, по определению (см. § 3), предел обратной последовательности серровских расслоений над компактными полиэдрами является стинродовским расслоением при условии, что обратная последовательность тотальных пространств сходится (см. § 7). Следующий пример показывает, что последнее предположение нельзя отбросить.

ПримеР 8.10. Пусть $X \subset \Sigma_{p} \times \Sigma_{p} \times I$ определен как объединение $\left[\Sigma_{p} \times\right.$ $\left.\Sigma_{p} \times \partial I\right] \cup\left[\Sigma_{p} \times \mathrm{pt} \times I\right] \cup\left[\mathrm{pt} \times \Sigma_{p} \times I\right]$. Таким образом, $X=\underset{\lim }{\longleftarrow}\left(\cdots \stackrel{p_{1}}{\longrightarrow} P_{1} \stackrel{f}{\rightarrow} P_{0}\right)$, где каждый $P_{i}=\left[S^{1} \times S^{1} \times \partial I\right] \cup\left[S^{1} \times \mathrm{pt} \times I\right] \cup\left[\mathrm{pt} \times S^{1} \times \overleftarrow{I]}\right.$ и каждое $f_{i}$ есть ограничение отображения $p \times p \times \mathrm{id}_{I}$ из $S^{1} \times S^{1} \times I$ в себя. Рассмотрим стинродовскую компоненту $h=(\alpha+\mathbb{Z}, \alpha+\mathbb{Z})$ в $\left(\mathbb{Z}_{p} / \mathbb{Z}\right)^{2} \simeq \pi_{0}(X)$, где $\alpha=\overline{\ldots \alpha_{1} \alpha_{0}} \in \mathbb{Z}_{p} \backslash \mathbb{Z}$ (ср. пример 8.6). Пусть $X^{h} \subset X$ - множество ее точек, т. е. объединение всех компонент линейной связности $X$, отображающихся в $h$. Пусть $\varphi: \widetilde{X} \rightarrow X-$ наложение, индуцированное из универсального накрытия $\widetilde{P}_{1} \rightarrow P_{1}$, и пусть $\varphi^{h}: \widetilde{X}^{h} \rightarrow X^{h}$ обозначает его ограничение на $\widetilde{X}^{h}:=\varphi^{-1}\left(X^{h}\right)$.

Мы утверждаем, что $\varphi^{h}$ не является стинродовским расслоением. В самом деле, рассмотрим пулбеки $\widetilde{P}_{i} \rightarrow P_{i}$ универсального накрытия полиэдра $P_{1}$. Мы можем отождествить $\widetilde{X}^{h}$ с обратным пределом $\mathbb{R} \times \mathbb{R} \times \partial I$ подполиэдров $\widetilde{P}_{i}^{h}:=$ $[\mathbb{R} \times \mathbb{R} \times \partial I] \cup\left[K_{i} \times I\right]$ полиэдров $\widetilde{P}_{i}$, где $K_{i}=\left[\left(\overline{\alpha_{i-1} \ldots \alpha_{0}}+p^{i} \mathbb{Z}\right) \times \mathbb{R}\right] \cup[\mathbb{R} \times$ $\left.\left(\overline{\alpha_{i-1} \cdots \alpha_{0}}+p^{i} \mathbb{Z}\right)\right]$. Заметим, что $\tilde{b}:=(0,0,0)$ и $\tilde{b}^{\prime}:=(0,0,1)$ принадлежат разным компонентам связности $\widetilde{X}^{h}$, но соответствующие собственные лучи $\tilde{b}_{[0, \infty)}$ 
и $\tilde{b}_{[0, \infty)}$ собственно гомотопны в телескопе $\widetilde{P}_{[0, \infty)}^{h}$. Эта собственная гомотопия проектируется в стинродовский путь $\ell:(I, \partial I) \times[0, \infty) \rightarrow\left(P_{[0, \infty)}, b_{[0, \infty)} \cup b_{[0, \infty)}^{\prime}\right)$, где $b=\varphi^{h}(\tilde{b})$ и $b^{\prime}=\varphi^{h}\left(\tilde{b}^{\prime}\right)$.

Если $\varphi^{h}$ - стинродовское расслоение, то существует сходящаяся (см. $\S 7$ ) обратная последовательность $\cdots \rightarrow \widehat{P}_{2}^{h} \rightarrow \widehat{P}_{1}^{h}$ с $\lim _{i}^{h}=\widehat{X}^{h}$ и сохраняющее уровни отображение $w_{[0, \infty)}: \widehat{P}_{[0, \infty)}^{h} \rightarrow P_{[0, \infty)}$ с $\underset{\lim w_{i}}{\longleftarrow}=\varphi^{h}$ такое, что, по доказательству теоремы $3.15(\mathrm{~b}), p_{[-3, \infty-3)}^{[0, \infty)} \ell$ поднимается в стинродовский путь $\hat{\ell}: I \times[0, \infty) \rightarrow \widehat{P}_{[0, \infty)}^{h}$ с началом в $\hat{b}_{[0, \infty)}$, где $\hat{b}_{i}$ обозначает образ $\tilde{b}$ в $\widehat{P}_{i}^{h}$. Этот $\tilde{\ell}$ должен заканчиваться в $\hat{b}_{[0, \infty)}^{\prime \prime}$, где $\hat{b}_{i}^{\prime \prime}$ обозначает образ в $\widehat{P}_{i}^{h}$ некоторого поднятия $\tilde{b}^{\prime \prime} \in \tilde{X}^{h}$ точки $b^{\prime}$. Тогда $\tilde{b}^{\prime \prime}$ и $\tilde{b}$ принадлежат одной и той же стинродовской компоненте $\widetilde{X}^{h}=\mathbb{R} \times \mathbb{R} \times \partial I$, что приводит к противоречию, поскольку $\tilde{b}=(0,0,0)$ и $\tilde{b}^{\prime}=(m, n, 1)$ для некоторых $m, n \in \mathbb{Z}$.

ЗАмЕчАниЕ. Пример 8.10 также показывает, что индуцированные включением отображения $\pi_{0}\left(\widetilde{X}^{h}\right) \rightarrow \pi_{0}(\widetilde{X})$ и $\check{\pi}_{0}\left(\widetilde{X}^{h}\right) \rightarrow \check{\pi}_{0}(\widetilde{X})$ могут быть неинъективны. Это может представлять интерес в связи с доказательством "легкой" (“только если") импликации в теореме 8.9 .

\section{Список литературы}

[1] S. A. Melikhov, Colored finite type invariants and a multi-variable analogue of the Conway polynomial, arXiv: math/0312007.

[2] D. P. Sinha, The topology of spaces of knots, arXiv: math/0202287.

[3] S. A. Melikhov, "A polynomial compactification of configuration spaces and resolution of the Thom-Boardman singularities" (to appear).

[4] R. Budney, J. Conant, K. P. Scannell, D. Sinha, "New perspectives on self-linking", Adv. Math., 191:1 (2005), 78-113; arXiv: math/0303034.

[5] I. Volić, "Configuration space integrals and Taylor towers for spaces of knots", Topology Appl., 153:15 (2006), 2893-2904; arXiv: math/0401282.

[6] I. Volić, "Finite type knot invariants and calculus of functors", Compos. Math., 142:1 (2006), 222-250; arXiv: math/0401440.

[7] R. Koytcheff, A homotopy-theoretic view of Bott-Taubes integrals and knot spaces, arXiv: abs/0810.1785.

[8] М.Н. Гусаров, "Вариации заузленных графов. Геометрическая техника $n$-эквивалентности", Алгебра и анализ, 12:4 (2000), 79-125; англ. пер.: M. N. Gusarov, "Variations of knotted graphs. The geometric technique of $n$-equivalence", St. Petersburg Math. J., 12 (2000), 569-604; http://www.math.toronto.edu/ drorbn/ Goussarov/.

[9] K. Habiro, "Claspers and finite type invariants of links", Geom. Topol., 4 (2000), 1-83; arXiv: math/0001185.

[10] S. A. Melikhov, E. V. Shchepin, The telescope approach to embeddability of compacta, arXiv: math/0612085.

[11] J.R. Isbell, Uniform spaces, Mathematical Surveys, 12, Amer. Math. Soc., Providence, RI, 1964.

[12] G. E. Bredon, Sheaf theory, 2nd ed., Grad. Texts in Math., 170, Springer-Verlag, New York, 1997; рус. пер. 1-го изд.: Г.Э. Бредон, Теория пучков, Наука, М., 1988. 
[13] J. Milnor, "On the Steenrod homology theory", Novikov conjectures, index theorems and rigidity, Vol. 2 (Oberwolfach, 1993), London Math. Soc. Lecture Note Ser., 226, Cambridge Univ. Press, Cambridge, 1995, 79-96; http://www. maths.ed.ac.uk/ aar/books/novikov2.pdf.

[14] Э. Спеньер, Алгебраическая топология, Мир, М., 1971; пер. с англ.: Е. Н. Spanier, Algebraic topology, McGraw-Hill, New York-Toronto-London, 1966.

[15] W.S. Massey, Homology and cohomology theory. An approach based on Alexander-Spanier cochains, Monogr. Textbooks Pure Appl. Math., 46, Dekker, New York-Basel, 1978; the author's guide to reading this book: W. S. Massey, "How to give an exposition of the Čech-Alexander-Spanier type homology theory", Amer. Math. Monthly, 85:2 (1978), 75-83.

[16] A. Borel, J. C. Moore, "Homology theory for locally compact spaces", Michigan Math. J., 7 (1960), 137-159.

[17] Е. Г. Скляренко, "Гомологии и когомологии общих пространств", Общая топология - 2, Итоги науки и техн. Соврем. пробл. матем. Фундам. направления, 50, ВИНИТИ, М., 1989, 129-266; англ. пер.: Е. G. Sklyarenko, "Homology and cohomology theories of general spaces", General topology II, Encycl. Math. Sci., 50, Springer, Berlin, 1996, 119-246.

[18] M. G. Barratt, J. W. Milnor, "An example of anomalous singular homology", Proc. Amer. Math. Soc., 13 (1962), 293-297.

[19] R. E. Williamson, Jr., "Cobordism of combinatorial manifolds", Ann. of Math. (2), 83 (1966), 1-33.

[20] Y. Kodama, "Fine movability", J. Math. Soc. Japan, 30:1 (1978), 101-116.

[21] S. Ferry, "A stable converse to the Vietoris-Smale theorem with applications to shape theory", Trans. Amer. Math. Soc., 261:2 (1980), 369-386.

[22] D. A. Edwards, H. M. Hastings, Čech and Steenrod homotopy theories with applications to geometric topology, Lecture Notes in Math., 542, Springer, 1976.

[23] J. Dydak, J. Segal, "Strong shape theory", Dissertationes Math. (Rozprawy Mat.), 192 (1981), 39 p.; announced in: J. Dydak, J. Segal, "Strong shape theory: a geometrical approach", Topology Proc., 3:1 (1978), 59-72; surveyed in: J. Dydak, "Strong shape theory, a survey of results", Proceedings of the International Conference on Geometric Topology, Vol. I (Warsaw, 1978), PWN, Warsaw, 1980, 121-126.

[24] Y. Kodama, J. Ono, "On fine shape theory", Fund. Math., 105 (1979), 29-39; Y. Kodama, J. Ono, "On fine shape theory. II", Fund. Math., 108:2 (1980), 89-98; Y. Kodama, "On fine shape theory. III", Glas. Mat. Ser. III, 16:2 (1981), 369-375.

[25] F. Cathey, "Strong shape theory", Shape theory and geometric topology (Dubrovnik, 1981), Lecture Notes in Math., 870, Springer, Berlin-New York, 1981, 215-238.

[26] Y. Iwamoto, K. Sakai, "Strong n-shape theory", Topology Appl., 122:1-2 (2002), 253-267.

[27] S. Mardešić, J. Segal, Shape theory. The inverse system approach, North-Holland Math. Library, 26, North-Holland, Amsterdam-New York, 1982.

[28] R. H. Fox, "On shape", Fund. Math., 74:1 (1972), 47-71.

[29] J. Dydak, J. Segal, Shape theory. An introduction, Lecture Notes in Math., 688, Springer-Verlag, Berlin, 1978.

[30] T. Porter, "Čech and Steenrod homotopy and the Quigley exact couple in strong shape and proper homotopy theory", J. Pure Appl. Algebra, 24:3 (1982), 303-312.

[31] J. Segal, S. Spież, B. Günther, "Strong shape of uniform spaces", Topology Appl., 49:3 (1993), 237-249.

[32] D. E. Christie, "Net homotopy for compacta", Trans. Amer. Math. Soc., 56 (1944), 275-308. 
[33] J. B. Quigley, "An exact sequence from the $n$th to the $(n-1)$ st fundamental group", Fund. Math., 77 (1973), 195-210.

[34] N. E. Steenrod, "Regular cycles of compact metric spaces", Ann. of Math. (2), 41:4 (1940), 833-851.

[35] L.S. Pontrjagin, "Über den algebraischen Inhalt topologischer Dualitätssätze", Math. Ann., 105:1 (1931), 165-205; рус. пер.: Л. С. Понтрягин, "Об алгебраическом содержании топологических теорем двойственности”, Избранные научные труды, т. 1, Наука, М., 1988, 65-105.

[36] D. Doitchinov, "Uniform shape and uniform Čech homology and cohomology groups for metric spaces", Fund. Math., 102:3 (1979), 209-218.

[37] T. Miyata, "Homology, cohomology, and uniform shape", Glas. Mat. Ser. III, 30:1 (1995), 85-109.

[38] V. Agaronjan, Yu. M. Smirnov, "The shape theory for uniform spaces and the shape uniform invariants", Comment. Math. Univ. Carolin., 19:2 (1978), 351-357.

[39] E. Čech, "Théorie générale de l'homologie dans un espace quelconque", Fund. Math., 19 (1932), 149-183.

[40] S. Lefschetz, Algebraic topology, Amer. Math. Soc. Colloq. Publ., 27, Amer. Math. Soc., New York, 1942.

[41] Н. Стинрод, С. Эйленберг, Основания алгебрачческой топологии, ИЛ, М., 1958; пер. с англ.: S. Eilenberg, N. Steenrod, Foundations of algebraic topology, Princeton Univ. Press, Princeton, NJ, 1952.

[42] Y. Kodama, "On embeddings of spaces into ANR and shapes", J. Math. Soc. Japan, 27:4 (1975), 533-544.

[43] J. Krasinkiewicz, "On a method of constructing ANR-sets. An application of inverse limits", Fund. Math., 92:2 (1976), 95-112.

[44] T. A. Chapman, L. C. Siebenmann, "Finding a boundary for a Hilbert cube manifold", Acta Math., 137:3-4 (1976), 171-208.

[45] Y. Kodama, J. Ono, T. Watanabe, "AR associated with ANR-sequence and shape", General Topology Appl., 9:2 (1978), 71-88.

[46] L. C. Siebenmann, "Chapman's classification of shapes: a proof using collapsing", Manuscripta Math., 16:4 (1975), 373-384.

[47] R. C. Lacher, "Cell-like spaces", Proc. Amer. Math. Soc., 20:2 (1969), 598-602.

[48] B. Günther, "Semigroup structures on derived limits", J. Pure Appl. Algebra, 69:1 (1990), 51-65.

[49] R. Geoghegan, J. Krasinkiewicz, "Empty components in strong shape theory", Topology Appl., 41:3 (1991), 213-233.

[50] J.B. Quigley, "Equivalence of fundamental and approaching groups of movable pointed compacta", Fund. Math., 91 (1976), 73-83.

[51] J.W. Grossman, "Homotopy classes of maps between pro-spaces", Michigan Math. J., 21:4 (1974), 355-362.

[52] T. Watanabe, "On a problem of Y. Kodama", Bull. Acad. Polon. Sci. Sér. Sci. Math. Astronom. Phys., 25:10 (1977), 981-985.

[53] A. K. Bousfield, D. M. Kan, Homotopy limits, completions and localizations, Lecture Notes in Math., 304, Springer-Verlag, Berlin-New York, 1972.

[54] K. Iriye, "The first derived functor of the inverse limit and localization", J. Pure Appl. Algebra, 173:1 (2002), 7-14.

[55] B. I. Gray, "Spaces of the same $n$-type, for all n", Topology, 5:3 (1966), 241-243.

[56] R. Geoghegan, "A note on the vanishing of lim"1", J. Pure Appl. Algebra, 17:1 (1980), 113-116.

[57] C. A. McGibbon, J. M. Møller, "On spaces with the same $n$-type for all $n$ ", Topology, 31:1 (1992), 177-201. 
[58] R. Geoghegan, Topological methods in group theory, Grad. Texts in Math., 243, Springer, New York, 2008.

[59] M. Artin, B. Mazur, Etale homotopy, Lecture Notes in Math., 100, Springer-Verlag, Berlin-New York, 1969.

[60] A. Koyama, "A Whitehead-type theorem in fine shape theory", Glas. Mat. Ser. III, 18:2 (1983), 359-370.

[61] Ю.Т. Лисица, "Теоремы Гуревича и Уайтхеда в сильной теории шейпов", Докл. АН СCCP, 283:1 (1985), 38-43; англ. пер.: Yu. Т. Lisitsa, "Hurewicz and Whitehead theorems in the strong shape theory", Soviet Math. Dokl., 32:1 (1985), 36-39.

[62] J. Keesling, "Algebraic invariants in shape theory", Topology Proceedings, Vol. 1 (Auburn, AL, 1976), Auburn Univ., Auburn, 1977, 115-124.

[63] L. C. Siebenmann, "Infinite simple homotopy types", Indag. Math., 32 (1970), 479-495.

[64] D. A. Edwards, R. Geoghegan, "Shapes of complexes, ends of manifolds, homotopy limits and the Wall obstruction", Ann. Math. (2), 101 (1975), 521-535.

[65] D. A. Edwards, R. Geoghegan, "The stability problem in shape, and a Whitehead theorem in pro-homotopy", Trans. Amer. Math. Soc., 214 (1975), 261-277.

[66] J. Dydak, "A simple proof that pointed FANR-spaces are regular fundamental retracts of ANR's", Bull. Acad. Polon. Sci. Sér. Sci. Math. Astronom. Phys., 25:1 (1977), 55-62.

[67] J. Dydak, "The Whitehead and the Smale theorems in shape theory", Dissertationes Math. (Rozprawy Mat.), 156 (1979), 55 p.

[68] Y. Kodama, "A characteristic property of a finite-dimensional pointed FANR", Japan J. Math. (N. S.), 4:2 (1978), 445-460.

[69] S. Ferry, A. Ranicki, "A survey of Wall's finiteness obstruction", Surveys on surgery theory, Vol. 2, Ann. of Math. Stud., 149, Princeton Univ. Press, Princeton, NJ, 2001, 63-79; arXiv: math/0008070.

[70] J. Dydak, "Local $n$-connectivity of quotient spaces and one-point compactifications", Shape theory and geometric topology (Dubrovnik, 1981), Lecture Notes in Math., 870, Springer, Berlin-New York, 1981, 48-72.

[71] J. Dydak, "Relations between homology and homotopy pro-groups of continua", The Proceedings of the 1981 Topology Conference (Blacksburg, VA, 1981), Topology Proc., 6, № 2, 1981, 267-278.

[72] S. Mardešić, T. B. Rushing, "Shape fibrations. I", General Topology Appl., 9:3 (1978), 193-215.

[73] F. Cathey, "Shape fibrations and strong shape theory", Topology Appl., 14:1 (1982), 13-30.

[74] С. А. Богатый, "Теорема Вьеториса для шейпов, обратные пределы и одна проблема Ю. М. Смирнова”, Докл. АН СССР, 211 (1973), 764-767; англ. пер.: S. Bogatyi, "The Vietoris theorem for shapes, inverse limits, and a certain problem of Ju. M. Smirnov", Soviet Math. Dokl., 14 (1973), 1089-1094.

[75] K. Kuperberg, "Two Vietoris-type isomorphism theorems in Borsuk's theory of shape, concerning the Vietoris-Cech homology and Borsuk's fundamental groups", Studies in topology (Charlotte, NC, 1974), Academic Press, New York, 1975, 285-314.

[76] S. Ferry, "Remarks on Steenrod homology", Novikov conjectures, index theorems and rigidity, Vol. 2 (Oberwolfach, 1993), London Math. Soc. Lecture Note Ser., 227, Cambridge Univ. Press, Cambridge, 1995, 148-166; http://www.maths.ed.ac.uk/ aar/books/novikov2.pdf.

[77] J.H.C. Whitehead, "A certain exact sequence", Ann. of Math. (2), 52:1 (1950), 51-110. 
[78] Y. Kodama, A. Koyama, "Hurewicz isomorphism theorem for Steenrod homology", Proc. Amer. Math. Soc., 74:2 (1979), 363-367.

[79] Ю. Кодама, “Обобщение подвижности и теорема об изоморфизме Гуревича для стинродовских гомологий”, УМH, 34:6 (1979), 51-52; англ. пер.: Y. Kodama, "Generalization of movability and Hurewicz's isomorphism theorem for Steenrod homology", Russian Math. Surveys, 34:6 (1979), 57-59.

[80] K. Kuperberg, "A note on the Hurewicz isomorphism theorem in Borsuk's theory of shape", Fund. Math., 90:2 (1976), 173-175.

[81] J. Dydak, "Concerning the abelization of the first shape group of pointed continua", Bull. Acad. Polon. Sci. Sér. Sci. Math Astronom. Phys., 24 (1976), 615-620.

[82] С.А. Мелихов, "Изотопическая и непрерывная реализуемость отображений в метастабильном ранге", Матем. сб., 195:7 (2004), 71-104; англ. пер.: S. A. Melikhov, "Isotopic and continuous realizability of maps in the metastable range", Sb. Math., 195:7-8 (2004), 983-1016.

[83] R. M. Vogt, "On the dual of a lemma of Milnor", Proceedings of the Advanced Study Institute on Algebraic Topology, Vol. III (1970), Various Publ. Ser., 13, Mat. Inst., Aarhus Univ., Aarhus, 1970, 632-648.

[84] K. Eda, K. Kawamura, "The singular homology of the Hawaiian earring", J. London Math. Soc. (2), 62:1 (2000), 305-310.

[85] K. Eda, K. Kawamura, "Homotopy and homology groups of the $n$-dimensional Hawaiian earring", Fund. Math., 165:1 (2000), 17-28.

[86] S. Zdravkovska, "An example in shape theory", Proc. Amer. Math. Soc., 83:3 (1981), 594-596.

[87] W. Hurewicz, "Homologie, Homotopie und lokaler Zusammenhang", Fund. Math., 25 (1935), 467-485.

[88] K. Borsuk, "On the n-movability", Bull. Acad. Polon. Sci. Ser. Sci. Math. Astronom. Phys., 20 (1972), 859-864.

[89] J. Dydak, J. Segal, "Local n-connectivity of decomposition spaces", Topology Appl., 18:1 (1984), 43-58.

[90] S. Ferry, "Homotoping ع-maps to homeomorphisms", Amer J. Math., 101:3 (1979), $567-582$.

[91] D. J. Garity, J.P. Henderson, D. G. Wright, "Menger spaces and inverse limits", Pacific J. Math., 131:2 (1988), 249-259.

[92] W. J. R. Mitchell, D. Repovš, E. V. Ščepin, "On 1-cycles and the finite dimensionality of homology 4-manifolds", Topology, 31:3 (1992), 605-623.

[93] K. Eda, K. Kawamura, "The surjectivity of the canonical homomorphism from singular homology to Čech homology", Proc. Amer. Math. Soc., 128:5 (2000), 1487-1495.

[94] O. Jussila, "On homology theories in locally connected spaces", Ann. Acad. Sci. Fenn. Ser. A I, 340 (1964), 15 p.; O. Jussila, "On homology theories in locally connected spaces. II", Ann. Acad. Sci. Fenn. Ser. A I, 378 (1965), 8 p.

[95] S. Mardešić, "Comparison of singular and Čech homology in locally connected spaces", Michigan Math. J., 6 (1959), 151-166.

[96] S. Buoncristiano, C. P. Rourke, B. J. Sanderson, A geometric approach to homology theory, London Math. Soc. Lecture Note Ser., 18, Cambridge Univ. Press, Cambridge-New York-Melbourne, 1976.

[97] K. Eda, U. Karimov, D. Repovš, "On (co)homology locally connected spaces", Topology Appl., 120:3 (2002), 397-401.

[98] J. Dydak, "Steenrod homology and local connectedness", Proc. Amer. Math. Soc., 98:1 (1986), 153-157.

[99] D. R. McMillan, Jr., "One-dimensional shape properties and three-manifolds", Studies in topology (Charlotte, NC, 1974), Academic Press, New York, 1975, 367-381. 
[100] N. Shrikhande, "Homotopy properties of decomposition spaces", Fund. Math., 116:2 (1983), 119-124.

[101] R. H. Fox, "Shape theory and covering spaces", Topology Conference (Blacksburg, VA, 1973), Lecture Notes in Math., 375, Springer, Berlin, 1974, 71-90.

[102] T. T. Moore, "On Fox's theory of overlays", Fund. Math., 99:3 (1978), 205-211.

[103] S. Mardešić, V. Matijević, "Classifying overlay structures of topological spaces", Topology Appl., 113:1-3 (2001), 167-209.

[104] M. F. Atiyah, G. B. Segal, "Equivariant $K$-theory and its completion", J. Differential Geometry, 3 (1969), 1-18.

[105] L. J. Hernández-Paricio, "Fundamental pro-groupoids and covering projections", Fund. Math., 156:1 (1998), 1-31.

[106] N. Brodskiy, J. Dydak, B. Labuz, A. Mitra, Rips complexes and covers in the uniform category, arXiv: abs/0706.3937.

[107] J. Vilímovský, "Uniform quotients of metrizable spaces", Fund. Math., 127:1 (1987), $51-55$.

[108] M. R. Bridson, A. Haefliger, Metric spaces of non-positive curvature, Grundlehren Math. Wiss., 319, Springer-Verlag, Berlin, 1999.

[109] V. Runde, A taste of topology, Universitext, Springer, New York, 2005.

[110] B. LaBuz, Inverse limits of uniform covering maps, arXiv: abs/0808.4119.

[111] В. М. Бухштабер, А. В. Шокуров, “Алгебра Ландвебера-Новикова и формальные векторные поля на прямой", Функи. анализ и его прил., 12:3 (1978), 1-11; англ. пер.: V. M. Bukhshtaber, A. V. Shokurov, "The Landweber-Novikov algebra and formal vector fields on the line", Funct. Anal. Appl., 12:3 (1978), 159-168.

[112] В. М. Бухштабер, "Группы полиномиальных преобразований прямой, неформальные симплектические многообразия и алгебра Ландвебера-Новикова", УМH, 54:4 (1999), 161-162; англ. пер.: V. M. Bukhshtaber, "Groups of polynomial transformations of a line, non-formal symplectic manifolds, and the Landweber-Novikov algebra", Russian Math. Surveys, 54:4 (1999), 837-838.

[113] И. К. Бабенко, С. А. Богатый, "О группе подстановок формальных целочисленных рядов”, Изв. РАН. Сер. матем., 72:2 (2008), 39-64; англ. пер.: I. K. Babenko, S. A. Bogatyi, "On the group of substitutions of formal power series with integer coefficients", Izv. Math., 72:2 (2008), 241-264.

[114] D. L. Johnson, "The group of formal power series under substitution", J. Austral. Math. Soc. Ser. A, 45:3 (1988), 296-302.

[115] J. Krasinkiewicz, "Continuous images of continua and 1-movability", Fund. Math., 98:2 (1978), 141-164.

[116] J. Krasinkiewicz, "On pointed 1-movability and related notions", Fund. Math., 114:1 (1981), 29-52.

[117] J. Krasinkiewicz, P. Minc, "Generalized paths and pointed 1-movability", Fund. Math., 104:2 (1979), 141-153.

\section{С. А. Мелихов (S. А. Melikhov)}

Математический институт им. В. А. Стеклова РАН

E-mail: melikhov@mi.ras.ru
Поступила в редакцию 17.03.2009 